\title{
Bibliographic Survey of Medium Energy Inclusive Reaction Data
}

\author{
E. D. Arthur \\ D. G. Madland \\ D. M. McClellan
}

\section{DISCLAIMER}

This report was prepared as an account of work sponsored by an agency of the United States Government. Neither the United States Government nor any agency thereof, nor any of their employees, makes any warranty, express or implied, or assumes any legal liability or responsibility for the accuracy, completeness, or usefulness of any information, apparatus, product, or process disclosed, or represents that its use would not infringe privately owned rights. Reference herein to any specific commercial product, process, or service by trade name, trademark, manufacturer, or otherwise does not necessarily consti' 'te or imply its endorsement, recommendation, or favoring by the United States Government or any agency thereof. The views and opinions of authors expressed herein do not necessarily state or reflect those of the United States Government or any agency thereof.
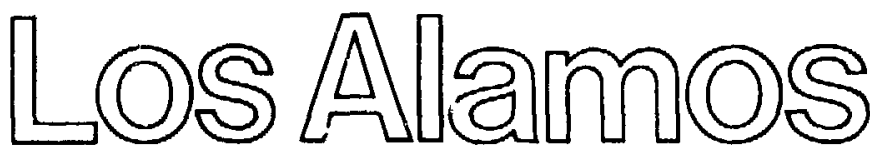
OVERVIEW AND APPROACH, $\ldots \ldots \ldots \ldots \ldots \ldots \ldots \ldots \ldots \ldots \ldots \ldots \ldots \ldots \ldots$

TABLE $I=$ PROTON-INDUCED REACTIONS $\ldots \ldots \ldots \ldots \ldots \ldots \ldots \ldots \ldots \ldots$

TABLE II - DEUTERON-INDUCED REACTIONS $\ldots \ldots \ldots \ldots \ldots \ldots \ldots \ldots$

TABLE III $=$ TRITON- AND ${ }^{3}$ He-INDUCED REACTIONS $\ldots \ldots \ldots \ldots \ldots \ldots \ldots 13$

TABLE IV - ALPHA-PARTICLE INDUCED REACTIONS $\ldots \ldots \ldots \ldots \ldots \ldots \ldots \ldots 121$

TABLE $v-6,7_{\text {Li }}$ INDUCED REACTIONS $\ldots \ldots \ldots \ldots \ldots \ldots \ldots \ldots \ldots \ldots$

TABLE VI - EXPERIMENTAL DATA REFERENCE TABLE FOR PROTON ELASTIC

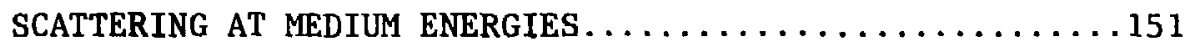

TABLE VII - EXPERIMENTAL DATA REFERENCE TABLE FOR PROTON TOTAL REACTION CROSS SECTIONS AT MEDIUM ENERGIES . . . . . . 155 


\title{
BIBLIOGRAPHIC SURVEY OF NEDIUM ENERGY INCLUSIVE REACTION DATA
}

\author{
by
}

E. D. Arthur, D. G. Madland, and D. M. McClellan

\section{ABSTRACT}

A bibliographic survey of inclusive reaction data (experimentil and theoretical) for several projectile types having energies between 50 and $1000 \mathrm{MeV}$ has been completed. Approximately one thousand references selected from this survey describe the current state of knowle:dge for particleinduced inclusive reaction data.

\section{OVERVIEW AND APPROACH}

This report provides results of a recently completed search of bibliographic information pertaining to currently available inclusive reaction data. Particularly emphasized were medium energy data describing experimental and theoretical results for particle and gamma-ray emission. Other types of information that were included pertain to the use of appropriate inedium-energy theoretical models and to experimental results for materials damage, nuclear recoils, and radiation effects produced by medium-energy projectiles.

The search covered data for the following projectiles: $p, d, t,{ }^{3} \mathrm{He},{ }^{4} \mathrm{He}$, and lithium ions, over an incident energy range extending from approximately 50 $\mathrm{MeV}$ to $1000 \mathrm{MeV}$. The search utilized the US Department of Energy RECON (Remote Console) bibliographic data system and spanned the time period from 1947-1985. Initially, more than 17000 possible entries were identified using key word techniques. Subsequently, approximately 1000 references were selected by scanning abstracts, and in some cases, text. These have been judged appropriate for describing the current state of knowledge concerning inclusive reaction data, both from an experimental and theoretical viewpoint. We have also included bibliographis information needed for assessment, validation, and refinement of nuclear model capabilities. 
For the selected bibliographic entries, the following is provided: reference, author(s), title, target materials, and descriptor(s) characterizing the content of the bibliographic entry. Tables I-V list such information for incident protons, deuterons, tritons, ${ }^{3} \mathrm{He}$, alpha-particles, and ${ }^{6,7} \mathrm{Li}$ ions .

The bibliographic entries in Tables $I-V$ follow a rough sequence where more recent data precedes older publications. Additionally, an attempt was made to place entries for higher energy data ( $100 \leqq E \leqq 1000 \mathrm{MeV}$ ) before those appropriate for lower energies. This ordering was followed for publications appearing between 1975-1985; but for entries occurring before 1975, such a grouping became more difficult. Particularly for data occurring between 1947-1960, no energy ranges could be specified, so that a mixture of higher and lower energy data results.

Finally, Tables VI and VII summarize selected references for proton elastic scattering and total reaction cross-section data. Such infolnation is provided because of the important constraints it imposes upon the severai theoretical models that are currently used to calculate proton-induced inclusive reaction data.

I1. OTHER SOURCES OF CHARGED-PARTICLE REACTION DATA

The following is a partial list of other sources of bibliographic data for charged-particle reaction data.

1. N. E. Holden, S. Ramavataram, C. L. Dunford, "Integral Charged-Particle Nuclear Data Bibliography," Brookhaven National Laboratory report BNL-NCS51771, First Edition (1984) and Supplement One (1985).

2. N. E. Holden and T. W. Burrows, "The Bibliography of Integral Charged Particle Nuclear Data," Brookhaven National Laboratory report BNL-NCS -50640 (1982), Fourth Edition, Supplement 2.

3. T. W. Burrows and G. Wyant, "The Bibliography of Integral Charged Particle Nuclear Data," Brookhaven National Laboratory report BNL-NCS-60640 (1981), Fourth Edition, Supplement 1.

4. T. W. Burrows and P. Dempsey, "The Bibliography of Integral Charged Particle Nuclear Data," Brookhaven National Laboratory BNL-NCS-50640 (1980), Fourth Edition (Archival Edition).

5. F. K. McGown and W. T. Milner, Reaction List for Charged-Particle-Induced Reactions. Atomic Data Nuclear Data Reprints 2 (Academic Press, New York, 1974).

6. K. A. Keller, et al., Q-Values and Excitation Functions of Nuclear Reactions. Landholt-Boernstein, Group I, Vol. 5 Parts A-C (Springer-Verlag, Berlin, 1973). 


\begin{tabular}{|c|c|c|c|}
\hline TARGET & TITLE & REFERENCE & DESCRIPTORS \\
\hline${ }^{208} \mathrm{~Pb}$ & $\begin{array}{l}\text { Relativistic vs Nonrelati- } \\
\text { vistic Description of Proton } \\
\text { Elastic Scattering in the } \\
\text { Energy Range } 200-500 \mathrm{MeV}\end{array}$ & $\begin{array}{l}\text { Phys Lett B152 } \\
\text { 319(1985) } \\
\text { R.Dymarz }\end{array}$ & $\begin{array}{l}\text { optical model, } \\
\text { Dirac equation }\end{array}$ \\
\hline${ }^{9} \mathrm{Be},{ }^{12} \mathrm{C}$ & $\begin{array}{l}\text { Contribution of Quasi-Electric } \\
\text { Mechanism to Inclusive Spectra } \\
\text { of Light Fragments Emitted to } \\
\text { the Back Hemisphere }\end{array}$ & $\begin{array}{l}\text { Bull Acad Sciences } \\
\text { USSR, Phys Series } \\
\text { 47,2130 (1983) } \\
\text { L.I.Galanina, } \\
\text { N.S.Zelenskaya }\end{array}$ & $\begin{array}{l}\text { knock-out } \\
\text { reactions }\end{array}$ \\
\hline $\mathrm{C}, \mathrm{Pb}, \mathrm{Ag}$ & $\begin{array}{l}\text { Production Mechanism of Back- } \\
\text { ward Energetic Protons Studied } \\
\text { from Two-Particle Correlations } \\
\text { in } 800 \mathrm{MeV} \text { Proton-Nucleus } \\
\text { Collisions }\end{array}$ & $\begin{array}{l}\text { Phys Rev C31, } 2168 \\
\text { (1985) } \\
\text { Y.Miake et al }\end{array}$ & $\begin{array}{l}\text { quasi elastic } \\
\text { scattering }\end{array}$ \\
\hline $\begin{array}{l}27 \mathrm{Al}, 197 \mathrm{Au} \\
6 \mathrm{Li}, 58 \mathrm{Ni} \\
28 \mathrm{Si}\end{array}$ & $\begin{array}{l}\text { Energetic Protons at Large } \\
\text { Angles in Coincidence with } \\
\text { Gamma Rays }\end{array}$ & $\begin{array}{l}\text { Workshop on } \\
\text { Coincident Particle } \\
\text { Emission from Conti- } \\
\text { nuum States, Bad Honnef, } \\
\text { W.Germany, INIS-MF- } \\
\text { 9280(1984) M.Avan et al }\end{array}$ & $\begin{array}{l}\text { inclusive } \\
\text { interactions }\end{array}$ \\
\hline s1v & $\begin{array}{l}51 \mathrm{~V}(\mathrm{p}, n)^{51} \mathrm{Cr} \text { Reaction at } \mathrm{E}_{\mathrm{p}}= \\
160 \mathrm{MeV}\end{array}$ & $\begin{array}{l}\text { Nucl Phys A427, } \\
332,(1984) \\
\text { J.Rapaport et al }\end{array}$ & neutron spectra \\
\hline $\begin{array}{l}27 \mathrm{Al}, 12 \mathrm{C} \\
63 \mathrm{Cu}, 4 \mathrm{He} \\
208 \mathrm{~Pb}, 118 \mathrm{Sn}\end{array}$ & $\begin{array}{l}\text { Energy Dependence of Nuclear } \\
\text { Reaction Cross Sections }\end{array}$ & $\begin{array}{l}\text { Phys Lett B146, } 370 \\
\text { (1984) N.H.Wong, } \\
\text { J.Huefner }\end{array}$ & $\begin{array}{l}\text { total cross } \\
\text { sections }\end{array}$ \\
\hline $27 \mathrm{Al}, \mathrm{Cu}$ & $\begin{array}{l}\text { Experimental Study of Direct } \\
\text { Knockout of Proton Pairs from } \\
\text { Nuclei by } 640-\mathrm{MeV} \text { Protons }\end{array}$ & $\begin{array}{l}\text { Sov J Nucl Phys } \\
40,17(1984) \\
\text { V.S.Nadezhdin et al }\end{array}$ & proton spectra \\
\hline $\begin{array}{l}56 \mathrm{Fe}, 51 \mathrm{~V} \\
75 \mathrm{As}, 59 \mathrm{Co} \\
63 \mathrm{Cu}\end{array}$ & $\begin{array}{l}\text { Systematics of Spallation } \\
\text { Yields with a Four-Parameter } \\
\text { Formula }\end{array}$ & $\begin{array}{l}\text { Rad Acta 35,121 } \\
\text { (1984) M.Foshina } \\
\text { et al }\end{array}$ & spallation \\
\hline $207,208 \mathrm{pb}$ & $\begin{array}{l}\text { Microscopic Analyses of Pro- } \\
\text { ton Scattering from Lead }\end{array}$ & $\begin{array}{l}\text { Nucl Phys A426,496 } \\
\text { (1984) L.Rikus,H.V. } \\
\text { Von Geramb }\end{array}$ & optical models \\
\hline
\end{tabular}




\section{$27 \mathrm{Al}_{2} 59 \mathrm{Co}$ $55 \mathrm{Mn}, 58 \mathrm{Ni}$ $48 \mathrm{Ti}, 51 \mathrm{~V}$}

$7 \mathrm{Li}$

${ }_{208}^{B i}$

$238 \mathrm{U}$

$27 \mathrm{Al}$, $197 \mathrm{Au}$, $6 \mathrm{Li}, 58 \mathrm{Ni}$

$58 \mathrm{Ni}, 16 \mathrm{O}$

$28 \mathrm{Si}, 90 \mathrm{Zr}$

$40,48 \mathrm{Ca}$

${ }^{12} \mathrm{C}, 64 \mathrm{Cu}$

$\mathrm{Pb}$

$27 \mathrm{Al}, 6 \mathrm{Li}$ $197 \mathrm{Au}, 58 \mathrm{Ni}$

$28 \mathrm{Si}$

$182 W$
Proton Induced Reactions on

$\mathrm{Ti}, \mathrm{V}, \mathrm{Mn}, \mathrm{Fe}, \mathrm{Co}$ and $\mathrm{Ni}$. Measurement and Hybrid Model Analysis of Integral Excitation Functions and Their Application in Model Calculation For the Production of Cosmogenic Nuclides

Activation Measurements of the $7 \mathrm{Li}(\mathrm{p}, \mathrm{n}){ }^{7 \mathrm{Be} \text { Reactions }}$ from $60-480 \mathrm{MeV}$

Inclusive Measurement of Quasi-free ( $p, x n)$ Charge Exchange Reactions on Bismuth from 62 to $800 \mathrm{MeV}$

Comparison of High-Energy Fission Models

$200 \mathrm{MeV}$ Proton Scattering on Nuclei, Energetic Proton Analysis at Large Angle

Neutron Deep-Hole States from the (p,pn) Reaction

Neutron-Knockout Reactions fromil $2 \mathrm{H}, 40 \mathrm{Ca}$ and ${ }^{48} \mathrm{Ca}$

Role of the Delta-Resonance in Intranuclear Cascades

Energetic Proton Analysis at Large Angle by $200 \mathrm{MeV}$ Proton Scattering on Nuclei: Inclusive Spectra; Proton-Gamma Coincidence Spectra

Inelastic Scattering of Intermediate Energy Protons from Tungsten
Koeln Univ, W.

Germany report

INIS-MF--9214

p.165(1983)

R.Stueck

Phys Rev C30, 1999

(1984) J.D'Auria

et al

Phys Rev C30,236

(1984)

J.M.D'Auria et al

Nucl Inst Meth

222,540(1984)

T.W.Armstrong

Thesis, Clermont-

Ferrand Univ

France(1983)

G.Chaigne

$J$ de Physique 45 ,

91(1984) J.W.

Watson

Nucl Phys A424,92

(1984) M.Ahmad

et al

Dubna report JINR-

R-2-83-768 p. 10

(1983) N.S.Amelin

Thesis, Clermont-

Ferrand Univ.

France(1984)

M.Al-Zoubidi

Tenth Nuclear

Physics Conf, Can-

berra, Aust

INIS-MF-9064,p.34

(1984) B.Lay knock-out

reactions

proton reactions

quasi-free reactions,

nuclear cascades

cascade theory, fission neutrons

knock-out reactions, quasielastic scattering

knock-out reactions

knock-out reactions, neutron spectra

nuclear cascades

gamma radiation, nuclear cascades

inelastic scattering 


\section{$\mathrm{Al}, \mathrm{Ca}, \mathrm{Fe}$}

$\mathrm{Mg}$

$40 \mathrm{Ca}, 56 \mathrm{Fe}$

$27 \mathrm{Al}, 58 \mathrm{Ni}$

$28 \mathrm{Si}, 6 \mathrm{Li}$

$27 \mathrm{Al}$,

209Bi

$58 \mathrm{Ni}, 90 \mathrm{Zr}$

\section{$\mathrm{Bi}, \mathrm{Pb}$}

$27 \mathrm{Al}$,

197Au

$27 \mathrm{Al}$

${ }^{197} \mathrm{Au}, 6 \mathrm{Li}$

$9 \mathrm{Be}$

Mo
Measurement of Cross Sections $\mathrm{fo}_{\mathrm{i}}-22 \mathrm{Na}, 20 \mathrm{Ne}, 22 \mathrm{Ne}$ and $36 \mathrm{Ar}-$ 42 $\mathrm{Ar}$ in the Spallaticn of $\mathrm{Mg}$,

$\mathrm{Si}, \mathrm{Ca}$ and $\mathrm{Fe}$. Production

Ratios of Some Cosmogenic

Ntclides in Meteorites

Nucleon Optical Potential

Backward Emission of Energetic Protons

New Developments in the Study of Continuum Spectra

Statistical Simulation of hadron-Nucleus and Light Nucleus-Nucleus Interaction Intranuclear Cascade Model

Evaluation of Computationai Models for Fission and Spallation Reactions used in Accelerator Breeding and Transmutation Analysis Code

Quasi-Free Scattering and Exciton Model Analysis at 200 $\mathrm{MeV}$

Backward Emission of Energetic Protons

Survey of Neutron Energy Spectra and Angular Distributions of the ${ }^{9} \mathrm{Be}(p, n){ }^{9} \mathrm{~B}$ Reaction for: Fast Neutron Radiotherapy

Medium-Energy Spallation Cross Sections. 2. Mo Iradiation with $800-\mathrm{MeV}$ Protons
$\mathrm{J}$ de Physique 45, 855(1984)F,Baros, S.Regnier

Nuovo Cimento $\underline{81,250}$

(1984) P.E.Hodgson

$\mathrm{J}$ de Physique 45 , 25(1984) M.Avan et al

$\mathrm{J}$ de Fhysique 45 , 3(1984)H.Machnet

Gosudarstvennyj

Inst. Moscow, ITEF-91(1983)

S.Y.Yader et al NTIS \#PC A03/MF A01

J.Nucl Sci \& Tech 20,511(1983)

Y.Nakahara

Phys Lett B138.39 (1984) H.Machner et al

Phys Rev C30,521

(1984)M.Avan

IAEA report INDC (NDS)--153/L p. 31 (1984) M.Aliab

Int J Appi.Rad \&

Iso.34,1631(1983)

P.M.Grant et al spallation, evaporation, nuclear cascades

proton interactions,nuclear cascades

proton reactions, precompoundnucleus, exclusive, inclusive interactions

hadron reactions, nuclear cascades

quasi-free reactions, inclusive interactions

inclusive interactions

neutron spectra, charge-exchiange reactions

spallation, fragments 
$84 \mathrm{Kr}$

$107_{\mathrm{Ag}}$

132Xe

$\mathrm{Pb}, \mathrm{U}$

27 Al, C, I,

$\mathrm{Fe}, \mathrm{Pb}, \mathrm{Nb}$

$\mathrm{Ta}, \mathrm{U}$

$12 \mathrm{C}$

${ }^{209} \mathrm{Bi}$

$238 \mathrm{U}$
Fragmentation Reactions on

Nuclei: Condensation of

Vapour or Shattering of Glass

Model Predictions of Neutron and Isotope Production from

Proton Induced Fission at

Higin Energies

Inclusive Quasi-Elastic

Knockout of Composite

Particles at Large Momentum

Transfers

Model Predictions of

Differential Neutron Produc-

tion Cross Sections for Lead

and Uranium Bombarded by

590-MeY Protons

Differential Neutron Production for $590 \mathrm{MeV}$ Protons

Analysis of Results on Elastic Scattering of Intermediate-

Energy Protons by Light and

Intermediate Nuclei

Case Study and Progress Report on Microscopic Analyses of Nucleon-Nucleus Scattering up to $400 \mathrm{MeV}$

Rapidity Dependence of

Entropy Production in

Proton- and Nucleus-Induced

Reactions on Heavy Nuclei

Medium Energy Proton Induced

Fission in Uranium-238 and

Bismuth-209
Phys Lett 136,15

(1984) J.Aichelin

Proc Int Conf on

Nuclear Data for

Sci \& Tech, Antwerp

p.391(Sept6-10,1982)

T.Armstrong et al

Sov J Nucl Phys

38,805:1983)

S.G.Kadmenskii,

Y.L.Ratis

Nucl Data Sci \&

Tech Int Conf

Antwerp(Sept6-

10,1982) p.387

T.W.Armstrong,

D.Filges

Nuc1 Data Sci \&

Tech Int Conf

Antwerp(Sept6-

10,198'2) p. 383

S.Cierjacks et al

Sov J Nucl Phys

38,706(1983)

N.I.Zaika, V.A.

Shityuk

Verhandlunger der

Deut Phys Gesel-

Ischaft(E.Ger.)

19,1047(1984)

H.V.Geramb,L.Rikus

Phys Rev C29, 1744

(1984)B.V.Jacak

et al

U of III,

Thesis (1983)

M.Ali proton reactions, inclusive interactions

fission, spallation, evaporation model, nuclear cascades

proton reactions, inclusive interactions

proton knockout reactions, spallation

spallation

elastic

scattering

nucleon reaction inelastic

scattering

heavy ion reaction, heavy nuclei, proton reactions, spallation, statistical models

spallation 
Ag

Inclusive Production of Isotopically Resolved Li through $\mathrm{Mg}$ Fragments by $480 \mathrm{MeV}$ p+Ag

$40,48 \mathrm{Ca}$

${ }^{59} \mathrm{Co}$

$175 \mathrm{Lu}$

$55 \mathrm{Mn}$

S1V

Fe,Ni,Ti

$58 \mathrm{Ni}$

160

$40 \mathrm{Ca}$

$208 \mathrm{pb}$
Neutron Knockout Reactions from $40 \mathrm{Ca}$ and $48 \mathrm{Ca}$

Measurement and Hybrid Model

Analysis of Integral Excitation Functions of Proton Induced Reactions up to $200 \mathrm{MeV}$ for $\mathrm{V}, \mathrm{Mn}, \mathrm{Co}$ and $45 \mathrm{MeV}$ for $\mathrm{Lu}$

Production of Cosmogenic Nuclides in Meteorites by Primary Galactic Particles: Cross Sections and Model Calculations

Three-Body Approach to the Multiple Scattering Direct Reactions: Pragmatic Application to the $58 \mathrm{Ni}(\mathrm{p}, 2 \mathrm{p}) 57 \mathrm{Co}$ Ce-rinuum

Neutron Induced Inclusive Light Fragment Production at Intermediate Energies

Microscopic and Macroscopic Aspects of $135 \mathrm{MeV}$ Proton Scattering from 160

Energy Dependence of NucleonNucleus Scattering
Phys Rev C29, 1806 (1984)R,E,L.Green et al

Thesis, Kent

State Univ(1982)

M.Ahmad

Verhandlungen der Deut Phys GeselIschaft(E.Ger.) 19,1022(1984)

F.Peiffer et al

J.Geophys Res $\underline{89}$, B673(1984) R. Michel,R.Stueck

Phys Rev C29, 1289 (1984)G.Ciangaru

et al

Proc Int Conf on High Energy Nuc Phys, Balatonfuered,Hungary (June 6-11,1983) p.133, J.Franz et al

Nucl Phys A413, 255(1984)K.Amos et al

Phys Rev C29,944 (1984)D.A.Sparrow proton reactions, fragmentation, energy spectra, evaporation

proton reactions, knock-out reactions

proton reactions, quasi-elastic scattering

proton reactions

inclusive reactions

inclusive interactions, proton reactions

proton reactions

Dirac equation, impulse approximation 


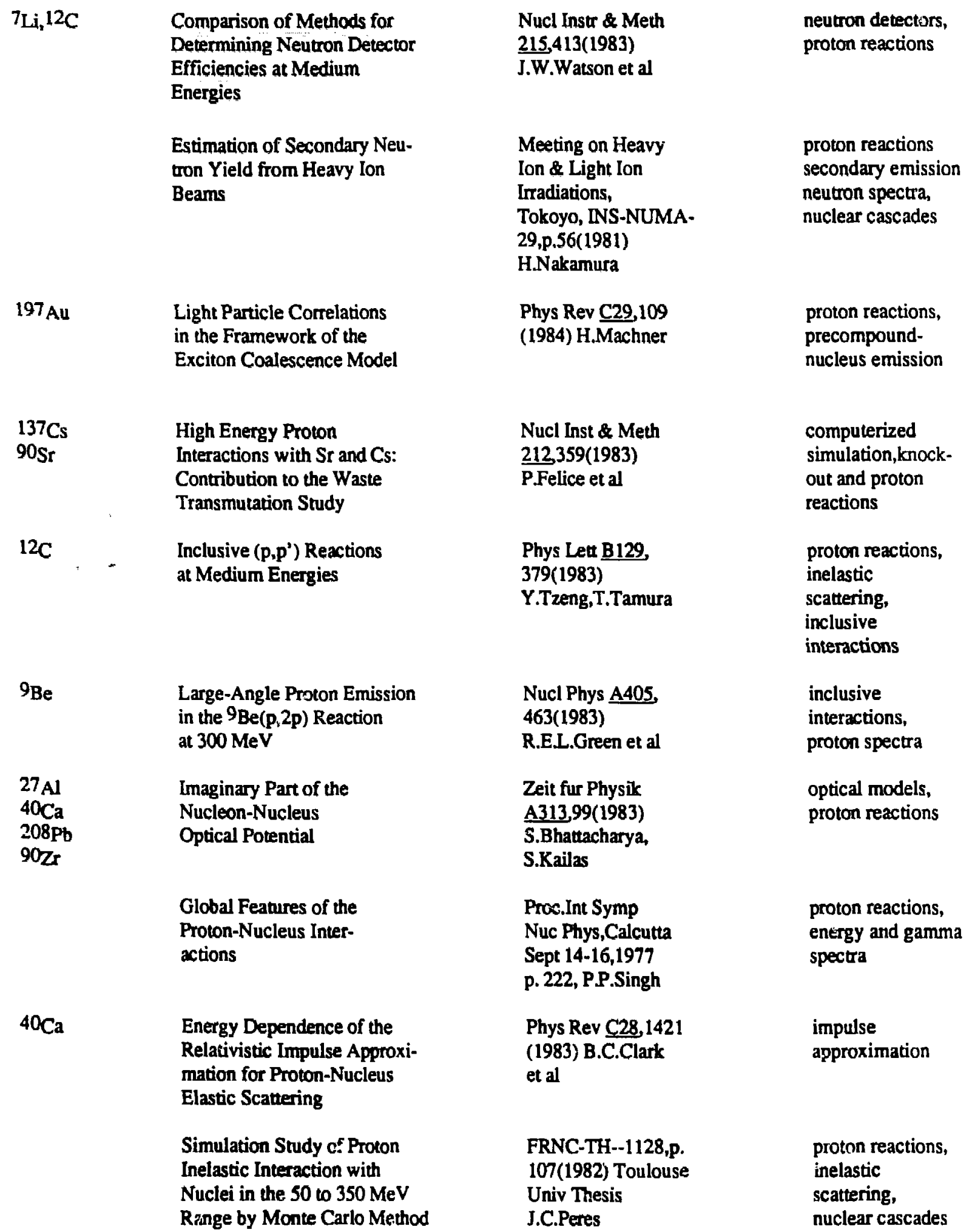


Table I (Cont.)

${ }^{232} \mathrm{Th}$
$235_{\mathrm{U}}$
$238 \mathrm{U}$
$208 \mathrm{~Pb}$

$\mathrm{Ce}, \mathrm{Eu}$

$165 \mathrm{Ho}$

$159 \mathrm{~Tb}$

$197 \mathrm{Au}$

$\mathrm{Bi}, 197 \mathrm{Au}$

$\mathrm{Ir}, 165_{\mathrm{Ho}}$

${ }^{169} \mathrm{Tm}$

$27 \mathrm{Al}, 209 \mathrm{Bi}$

$58 \mathrm{Ni}, 90 \mathrm{Zr}$

$28 \mathrm{Si}$

${ }^{40} \mathrm{Ca}$

$90 \mathrm{Zr}$

$40 \mathrm{Ca}$

$209 \mathrm{~Pb}$

$27 \mathrm{Al}$
Comparison of the (p,xn)Cross

Sections from 238U,235U, and

232Th Targets Irradiated with

$200 \mathrm{MieV}$ Protons

Measurement of the Average

Neutron Yield from $250 \mathrm{MeV}$

Protons Absorbed in a Lead

Target

$190 \mathrm{MeV}$ Proton-Induced Symme-

tric and Asymmetric Fission

Yield of Deep Spallation

Products of Medium to Heavy

Mass Targets Bombarded with 480-MeV Protons

Energy and Angular Distribution of Neutrons from $90 \mathrm{MeV}$ Proton and 140 MeV-Alpha Parícle Bombardment of Nuclei

Estimation of the Secondary Particle Production Time in Inelastic Light Nuclei

Interactions

Nonlocal and Local Equivalent Microscopic Optical Potentials

Giant Spin-Isospin Vibrations and the Continuem Spectra from the $(p, n)$ Reaction at Forward Angles

Dirac-Equation Impulse Approximation for Intermediate Energy Nucleon-Nucleus Scattering

Energy Deposition in Intermediate Energy NucleonNucleus Collisions

Inclusive Proton Spectra and Total Reaction Cross Sections for Proton-Nucleus Scattering at $800 \mathrm{MeV}$
1EEE Trans.Nuc

proton reactions

Sci 30,1153(1983)

Y.Y.Chu,M.L.Zhou

Zeit Physik A311, 363(1983)

Yu. Ryabov et al

Phys Rev $\underline{\text { C28, }}, 276$

(1983) F.D.

Becchetti et al

CTOM- $44886, p .171$

(1979) Simon Fraser

Univ, British Columbia, Thesis, H.P.E.Dautet

Phys Rev C28, 105

(1983) A.M.Kalend

et a!

proton reactions neutron spe: tra

Yad Fiz 36,8,390

(1982)

V.M.Dmitriev

et al

Phys Rev $\mathrm{C} 27,2466$

(1983) W.Bauhoff

Nuc Phys A395, 189

(i983) T.Izumoto

Phys Rev Lett 50,

1644(1983)

B.C.Clark et al

Phys Rev Lett 50 ,

1648(1983)

K.Kwiatkowski

Thesis (1981)

U.of Texas,Austin

J.A.McGill impulse

approximation

particle

production

proton reactions, optical models

neutron spectra

nuclear cascades, precompound tucleus emission

impulse approximation, inelastic scattering,proton spectra 


$\begin{array}{ll}58 \mathrm{Ni} & \begin{array}{l}\text { Continuum Spectrum in the } \\ \text { Quasifree (p,2p) Scattering }\end{array} \\ 27 \mathrm{Al}, 24 \mathrm{Mg} & \begin{array}{l}\text { Proton-Garnma Coincisience } \\ \text { Experiment on Medi n Mass } \\ \text { Nuclei at } 400 \mathrm{MeV} \text { and Study } \\ \text { of Reaction Mechanisms }\end{array}\end{array}$

2?Al,C $\mathrm{Cu}, \mathrm{Sn}, \mathrm{Ta}$

160

$40 \mathrm{Ca}, 12 \mathrm{C}$

$24 \mathrm{Mg}$

$\mathrm{Al}, \mathrm{As}, \mathrm{Br}$,
$\mathrm{Rb}, \mathrm{Y}$

$27 \mathrm{Al}, 40 \mathrm{Ca}$

$12 \mathrm{C}, 208 \mathrm{~Pb}$

Neutron Production from

Protons and Deuterons

Simulation of Inelastic

Collisions of Light Nuclei

Proton-Nucleus Total Inelastic Cross Sections: An Empirical Formula ror $E>10 \mathrm{MeV}$

Inclusive Charged Particle Spectrum from the Interaction of Nuclei with Intermediate Energy Protons

Studies of (p,n) Reactions at Intermediate Energies

Shell Effect Role in Nucleus

Fission by Fast Protons

Cross Sections for Reactions Rubidium and Yttrium

Reai Part of the Nucleon-

Nucleus Optical Potential
Phys Rev C27, 1360

(1983)

G.Ciangaru et al

PCCF-T-81-02

(1981) Clermont-

Ferrand Univ.

France,Thesis

A.Baldit

DLNUC/TM-60A

(1982) Daresbuy $y$

Lab(UK) report

(1982) R.Ryder with 593 and $540 \mathrm{MeV}$ Protons in Aluminium, Arsenic,Bromine,
Thesis(1980)

Kent State Univ

Ohio, J.N.Knudson

Sov J Nucl Phys

Sov Phys Usp. 31 ,

119(1982)

V.M.Dmitriev

Astrophy J. 51 ,

271(1983)

J.R.Letaw et al

Thesis(1981)

Northwestem U

Evanston,Il.

T.Chen

35,5,1131(1982)

M.M.Nesterov et al

Int J Applied Rad and Isotopes(England) $33,9,725$

(1982) A.Gruetter

Zeit fur Physik

A309,325(1983)

S.Bhattacharya,

S.Kailas quasi-free reactions, precompound-nucleus emission

quasi-ulastic scattering, nuclear cascades

spallation, neutron spectra

inelastic scattering

inelastic scattering

heavy nuclei, intermediate, light nuclei, inclusive interactions

charge-exchange reactions

fission, energy dependence

optical models 
Table I (Cont.)

\begin{tabular}{|c|c|}
\hline $\begin{array}{l}27 \mathrm{Al}, 208 \mathrm{Fb} \\
58,62 \mathrm{Ni}\end{array}$ & $\begin{array}{l}\text { Inclusive Proton Reactions } \\
\text { at } 164 \mathrm{MeV}\end{array}$ \\
\hline $235,238 \mathrm{U}$ & Comparison of the $(p, x n)$ \\
\hline $232 \mathrm{Th}$ & $\begin{array}{l}\text { Cress Sections from } 238 \mathrm{U}, 235 \mathrm{U} \text {, } \\
\text { and } 232 \mathrm{Th} \text { Targets Irradiated } \\
\text { with } 200-\mathrm{MeV} \text { Protons }\end{array}$ \\
\hline $\mathrm{Au}$ & $\begin{array}{l}\text { Study of the Pre-Equilibrium } \\
\text { Emission Following the Bom- } \\
\text { bardment of Nuclei with } \\
200 \mathrm{MeV} \text { Protons }\end{array}$ \\
\hline $235,238 \mathrm{U}$ & $\begin{array}{l}\text { Comparison of High-Energy } \\
\text { Fission Models for sie HETC } \\
\text { Transpor Code }\end{array}$ \\
\hline
\end{tabular}

$233,238 \mathrm{U}$

$\mathrm{Pb}$

${ }^{40,48} \mathrm{Ca}$

${ }^{27} \mathrm{Al}, 24 \mathrm{Mg}$ $60 \mathrm{Ni}, 28 \mathrm{Si}$

C

Neutron Production Yields and Spectra from $590 \mathrm{MeV}$ Proton Bombardment of Thick Uranium

High Energy Particle Spectra froin Spallation Targets

Single Particle Intranuclear "Cascade" and Pion Production Neutron Knockout Reactions at $149.5 \mathrm{MeV}$

Study $0^{6}$ the Reactions of 400 MeV Protons on Intermediate Coincidence Experiment

Quasifree Knockout of Proton
Phys Rev C26,2424

(1982) R.E. Segel

et al

Brookhaven report

BNL-32166(1982)

Y.Y.Chu,M.L.Zhou

Julich report

JUEL-SPEZ-146 p.33

(1982) J.P.Didelez

Julich report JUELCONF-45 Pt 1,p.281, Pt 2, p.299(1981)

Proc 5th Mtg of the Int. Collab on Advanced Neutron Sources, T.W.Armstrong et al

Julich report

${ }^{40} \mathrm{Ca}$ (p,pn), ${ }^{39} \mathrm{Ca}$ and ${ }^{48} \mathrm{Ca}$ (p,pn) Mass Nuclei by a Proton-Gamma Pairs from Carbon
JUEL-CONF-45,p.333

(1981) F.Raupp et al

Julich report

JUEL-CONF-45,p215

(1981) G.S.Bauer,

D.Filges

Phys Rev C26, 1558

(1982)D.G.Long,

M.M.Sternheim

Phys Rev C26,961 (1982)J.W.Watson

et al

Nuc Phys A384, 323 (1982)G.Landaud et al

Int Conf High

Energy Physics \& Nucl Structure Versailles,France (7-6-81)p.560 V.I.Komarov, G.E.Kosarev inclusive interactions, evaporation model

knock-out reactions

energy spectra

fission

spallation, neutron spectra

spallation

particle production, nuclear cascades

knock-out reactions

inclusive interactions

quasi-free reactions 


\begin{tabular}{|c|c|c|c|}
\hline $6 \mathrm{Li}$ & $\begin{array}{l}\text { Development of Intranuclear } \\
\text { Correlations in the Diffrac- } \\
\text { tive Scattering Cross Sections } \\
\text { of Fast Hadrons by Nuclei }\end{array}$ & $\begin{array}{l}\text { 32nd Conf on Nucl } \\
\text { Sper.t and Nucl } \\
\text { Stracture, Kiev } \\
\text { (1982)p.226 } \\
\text { V.V.Goryachij et al }\end{array}$ & $\begin{array}{l}\text { inelastic } \\
\text { scattering, } \\
\text { cluster model }\end{array}$ \\
\hline $\begin{array}{l}154 . \mathrm{Sm} \\
1\urcorner 5 \mathrm{Yb}\end{array}$ & $\begin{array}{l}\text { Microscopic Analysis of Proton } \\
\text { Scattering on Deíormed Nuclei } \\
\text { at Intermediate Energies }\end{array}$ & $\begin{array}{l}\text { 9th Int Conf High } \\
\text { Energy Physics \& } \\
\text { Nucl.Structure(1981) } \\
\text { p.152 Versailles, } \\
\text { France,J.Labarsouque }\end{array}$ & Glauber Theory \\
\hline $\begin{array}{l}\text { In,Sm } \\
\mathrm{Ag}, \mathrm{Sn}\end{array}$ & $\begin{array}{l}\text { Anisotropy and Cross Section } \\
\text { of Fission of Light Nuclei } \\
(100<\mathrm{A}<=150) \text { by } \\
\text { Protons with Energies } 155 \\
\text { and } 200 \mathrm{MeV}\end{array}$ & $\begin{array}{l}\text { Sov J Nucl Phys } \\
\text { 35,328(1982) } \\
\text { O.E.Shivaev et al }\end{array}$ & \\
\hline $\mathrm{Rb}, \mathrm{Br}$ & $\begin{array}{l}\text { Medium-Energy Spallation } \\
\text { Cross Sections. 1. RbBr } \\
\text { Irradiation with } 800-\mathrm{MeV} \\
\text { Protons }\end{array}$ & $\begin{array}{l}\text { Int J Applied Rad \& } \\
\text { Isotopes 33,41 } \\
\text { (i982) P.M. Grant } \\
\text { et al }\end{array}$ & \\
\hline $58 \mathrm{Ni}$ & $\begin{array}{l}\text { Effects of Nucleon Corre- } \\
\text { lations and Multi-Step } \\
\text { Transitions in Cross Sections } \\
\text { of Fast Proton-Nucleus } \\
\text { Inelastic Scattering }\end{array}$ & $\begin{array}{l}\text { Yad Fiz 34,9,691 } \\
\text { (1981) } \\
\text { G.D.Alkhazov }\end{array}$ & $\begin{array}{l}\text { heavy ruclei, } \\
\text { intermediate } \\
\text { nuclei }\end{array}$ \\
\hline $\begin{array}{l}\mathrm{Al}, \mathrm{Be} \\
\mathrm{C}, \mathrm{Cu}\end{array}$ & $\begin{array}{l}\text { General Features of Total } \\
\text { Hadron-Nucleus Reaction Cross } \\
\text { Sections }\end{array}$ & $\begin{array}{l}\text { F., Phys Acta } \\
54,3,432(1982) \\
\text { H.O.Meyer }\end{array}$ & proton reactions \\
\hline \multirow[t]{2}{*}{$238 \mathrm{U}$} & $\begin{array}{l}\text { Measurement of Energy Deposi- } \\
\text { ted by Charged-Particle } \\
\text { Beams in Composite Targets }\end{array}$ & $\begin{array}{l}\text { Lawrence Livermore } \\
\text { Lab. report } \\
\text { UCRL-52878(1980) } \\
\text { E.Farley et al }\end{array}$ & $\begin{array}{l}\text { lucite, } \\
\text { polyethylenes, } \\
\text { cascade showers }\end{array}$ \\
\hline & $\begin{array}{l}\text { Elastic Scattering in Intra- } \\
\text { nuclear Cascade Model }\end{array}$ & $\begin{array}{l}\text { Dubna report } \\
\text { JINR-R-2-81-366 } \\
\text { (1981) N.S.Amelin }\end{array}$ & nuclear cascades \\
\hline $\begin{array}{l}2.08 \mathrm{~Pb} \\
90 \mathrm{Zr} \\
116 \mathrm{Sn}\end{array}$ & $\begin{array}{l}\text { Excitation of the Giant- } \\
\text { Resonance Continuum with } \\
\text { Intermediate-Energy Protons }\end{array}$ & $\begin{array}{l}\text { Phys Rev Lett } 48 \text {, } \\
\text { 789(1982) J.M.Moss } \\
\text { et al }\end{array}$ & $\begin{array}{l}\text { quasi-free } \\
\text { reactions }\end{array}$ \\
\hline $62 \mathrm{Ni}$ & $\begin{array}{l}\text { Particle-Camma Ray Coincidence } \\
\text { Studies on } 62 \mathrm{Ni}\end{array}$ & $\begin{array}{l}\text { Indiana } U \\
\text { Thesis(1981) } \\
\text { J.D.Wiggins,Jr. }\end{array}$ & gamma spectra \\
\hline
\end{tabular}


Table I (Cont.)

$27 \mathrm{Al}, \mathrm{Cu}$

In, $\mathrm{Pb}, \mathrm{U}$

$12 \mathrm{C},{ }^{64} \mathrm{Cl}$

${ }^{12 C},{ }^{64} \mathrm{Cu}$

$6 \mathrm{Li}$

$\mathrm{Pb}$

$27 \mathrm{Al}^{12} \mathrm{C}$

${ }^{64} \mathrm{Cu}$

$207 \mathrm{~Pb}$

$27 \mathrm{Al},{ }^{9} \mathrm{Be}$

$\mathrm{C}, \mathrm{Cu}, \mathrm{Fe}$

$\mathrm{Ge}$

$107 \mathrm{Au}$

$40 \mathrm{Ca}$

$238 \mathrm{U}$
Determination of the Differ-

ential Cross Section for the Reaction X(p,n)Y Using $800 \mathrm{MeV}$ Proton Bombardment of $\mathrm{Al}, \mathrm{Cu}, \mathrm{In}, \mathrm{Pb}$, and $\mathrm{U}$

Energetic Particles Emitted from Energetic Nuclear Reactions

Model of Intranuclear Cascade with Account for Resonances

Scattering of $136 \mathrm{MeV}$ Protons from $6 \mathrm{Li}$

Neutron and Charged Particle Production Yields and Spectra from Thick Metal Targets by $590 \mathrm{MeV}$ Protons

Isobaric Model in Intranuclear Cascade Method

Study on the Neutron Yield from Thick Targets under the Action of $645 \mathrm{MeV}$ Protons

Proton Loss by Nuclear Inelastic Interactions in Germanium

Self-Consistent Method for Calculating the Evaporation of Heavy Fragments Following Energetic Initial Reactions

Model Independent Optical Potential for Protons at $\mathbf{1 5 5}$ $\mathrm{MeV}$

Analysis of the Yield of Neutrons Produced by HighEnergy Protons
Kansas State U

neutron spectra

Thesis(1980)

S.D.Howe

Zeit fur Physik

A306,177(1982)

A.H.Elin et al

Dubna report

JWNR-2-80-661

(1980)N.S.Amelin

Nucl Phys $\underline{A} 372$,

117(1981)

R.S.Henderson et al

Karlsruhe report

KFK-3097-B(1980)

S.Cierjacks et al

Bull Acad Sci

(USSR) $45,1,165$

(1981) V.E.Bunakov

et al

Dubna report

JINR-18-80-540

(1980) A.Y. Serov et al

Nucl Instr \& Meth

$188,465(1981)$

R.F.Carlson et al

Nucl Instr \& Meth

185, 195(1981)

E.L.Green.

R.G.Korteliing

Zeit fur Physik

A300,247(1981)

I.Brissaud

Sov At Energy 50, 150(1981)

V.S.Barashenkov, S.Y.Shmakov inclusive interactions, energy spectra

proton reactions, nuclear cascades

inelastic

scattering

neutron spectra

proton reactions, inclusive interactions

neutron emission

inelastic scattering

evaporation model, compound-nucleus reactioris

optical models

nuclear reaction yield, gamma radiation 
$\mathrm{Pb}$

$6 \mathrm{Li}$

$\begin{array}{ll}\mathrm{Al}, \mathrm{Be}, \mathrm{C} & \begin{array}{l}\text { Two-Particle Differential } \\ \text { Cross Sections of Proton } \\ \text { Emission in Proton-Nucleus } \\ \text { Interactions at } 640 \mathrm{MeV}\end{array} \\ 12 \mathrm{C} & \begin{array}{l}\text { Proton-Proton Correlations } \\ \text { in High Energy Nuclear } \\ \text { Collisions }\end{array} \\ 58,60 \mathrm{Ni} & \begin{array}{l}\text { Elastic and Inelastic } \\ \text { Scattering of 178 MeV }\end{array} \\ & \begin{array}{l}\text { Protons from 58Ni and 60Ni } \\ 27 \mathrm{Al}, 58 \mathrm{Ni}\end{array} \\ 208 \mathrm{~Pb} & \begin{array}{l}\text { Quasi-Free Proton-Scattering } \\ \text { at 164 MeV }\end{array} \\ & \\ 232 \mathrm{Th} & \text { Main Aspects of Proton Linear } \\ 235,238 \mathrm{U} & \begin{array}{l}\text { Accelerators for Nuclear Fuel } \\ \text { Production }\end{array}\end{array}$

$181 \mathrm{Ta}$

$209 \mathrm{Bi}$

$197 \mathrm{Au}$

$12 \mathrm{C}, 35 \mathrm{Cl}$

$63 \mathrm{Cu}, 19 \mathrm{~F}$

$208 \mathrm{~Pb}$
Angular and Depth Dependent

Neutron Yields and Spectra

from $590 \mathrm{MeV}(\mathrm{p}, \mathrm{n})$ Reactions

in Thick Lead Targets

Quasifree A(p,p,d)B Large-

Angle Scattering at Inter-

mediate Energies

Probability of Nuclear Fission

by Intermediate Energy

Particles

Emission in Proton-Nucleus

Elastic and Inelastic

Scattering of $178 \mathrm{MeV}$

Quasi-Free Proton-Scattering at $164 \mathrm{MeV}$

Production

Neutron Production by Protons (190 to $268 \mathrm{MeV}$ ) in a WaterCooled Tantalum Target

Studies of Intermediate

Energy Fission

One- and Two-Proton Inclusive Spectra in $800 \mathrm{MeV}$ ProtonNucleus Collisions and the Mean Free Path of Protons in Nuclei
Proc 5th All-

Union Conf Neutron

Physics, p.269(1980)

S.Cierjacks

$\mathrm{J}$ of Physics G7

1045(1981)

L.Vegh

Yad Fiz 32,322

(1980) A.S. I'Inov

et al

Yad Fiz $\underline{32}, 313$

(1980)

V.I.Komarov

Phys Lett B103,264

(1981) J.Knoll,

J.Randrup

Nucl Phys A365,426

(1981)

A.Ingemarsson et al

Phys Lett B103, 192

(1981) T.Chen

et al

Proc 6th All-Union:

Conf Charged Par-

ticles Accelerators

V.1, p.241(1979)

B.P.Murin, A.P.Fedotov

Oak Ridge report

ORNLTM-7949(1981)

R.G.Alsmiller et al

Thesis(1980)

S.Fraser Univ. (BC)

D.M.G. Dzutet

Phys Lett B100, 121

(1981) I.Tanihata

et al charge-exchange

reactions

quasi-free reactions, knock-out reactions

fission,cascade theory

cascade theory

inclusive interactions, cascade theory

elastic and inelastic scattering

quasi-free reactions

linear accelerators

neutron spectra

inclusive interactions 
Table I (Cont.)

$\begin{array}{ll}\text { C.Pb } & \text { Hadron Cascades in Thick } \\ \text { Materials Pt.1 Monte-Carlo } \\ \text { Method for Simulation of } \\ \text { Cascades }\end{array}$

$27 \mathrm{Al}$ $181 \mathrm{Ta}$

Au, Ho, Re

$\mathrm{Ta}, \mathrm{Tl}$

Ce.Yb

$\mathrm{C}, \mathrm{N}, \mathrm{O}$

${ }^{12} \mathrm{C}$

238U
Proton-Inclusive Cross

Sections from $000 \mathrm{MeV}$ Proton-

Nucleus Reactions

Study on the Secondary Radiation Field behind the Lead Atsorber Exposed to the $640 \mathrm{MeV}$ Proton Beam

Yield of Deep Spallation Products of Medium to Heavy Mass Targets Bombarded with $480 \mathrm{MeV}$ Protons

Mass Distributions in the Fission of Medium-Heavy and Light Nuclei

Specific Features of QuasiElastic Knock-Out of Slow(E< or approximately $10 \mathrm{MeV}$ ) Protons from C, N, O Nuclei by High Energy Protons

${ }^{12} \mathrm{C}(\mathrm{p}, \mathrm{n}){ }^{12} \mathrm{~N}$ Reaction at 120 , 160 , and $200 \mathrm{MeV}$

Delayed-Neutron Fraction in a Pulsed Spallation Neutron Source

Two-Particle Differential Cross Sections for Proton Emission in Proton-Nucleus Interactions at $640 \mathrm{MeV}$

Test of Models for Inclusive Production of Energetic Light Fragments at Intermediate Energies

Mechanisms for (p,p') Reactions in the Continuum
Nucl Inst \& Meth 179,77(1981)

M.Nieminen, J.J.

Torsti

Nucl Phys A352, 485(1981)

K.R.Cordell

Dubna report JINRR-16-12765(1979)

A.Y.Serov et al

Can J Phys $\underline{58,891}$ (1980) H.Dautet et al

Phys Rev C24,769 (1981)

H.Gustafsson et al

Yad Fiz 31,569

(1980)

A.A.Levkovskii, E.F. Dreiman

Phys Rev C24,335

(1981)

J.Rapaport et al

Nucl Instr \& Meth 175, 287(1980)

J.M.Carpenter

Sov J Nucl Phys 32,161(1980)

V.I.Komarov et al

Phys Rev C23,2788 (1981) D.H.Boal et al

Phys Lett B91,169 (1980) J.R.Wu knock-out reactions, neutron spectra

inclusive interactions

nuclear cascades, proton spectra

spallation

fission

proton spectra, quasi-elastic scattering

impulse approximation

spallation

inelastic scattering, quasi-free reactions, cascade theory

evaporation model,inclusive interactions

inelastic scattering, proton spectra 


\begin{tabular}{|c|c|c|c|}
\hline $\mathrm{La}$ & $\begin{array}{l}\text { Calculation of Spallation } \\
\text { Yields: Application to the } \\
\text { Spallation of Lanthanum } \\
\text { Induced by } 600 \mathrm{MeV} \text { Protons }\end{array}$ & $\begin{array}{l}\text { Phys Rev C23,2129 } \\
\text { (1981) G.Rudstam, } \\
\text { E.Hagebo }\end{array}$ & $\begin{array}{l}\text { evaporation model } \\
\text { nuclear cascades }\end{array}$ \\
\hline $40 \mathrm{Ca}$ & $\begin{array}{l}\text { Dirac Optical Model Analysis } \\
\text { of } 40 \mathrm{Ca} \text { Elastic Scattering at } \\
180 \mathrm{MeV} \text { and the Wine-Bottle- } \\
\text { Bottom Shape }\end{array}$ & $\begin{array}{l}\text { Phys Rev C23,1949 } \\
\text { (1981) L.G.Amold } \\
\text { et ai }\end{array}$ & $\begin{array}{l}\text { elastic } \\
\text { scattering, } \\
\text { Dirac equation }\end{array}$ \\
\hline $\begin{array}{l}209 \mathrm{Bi} \\
207 \mathrm{pt} \\
239 \mathrm{Pu}\end{array}$ & $\begin{array}{l}\text { Nuclear Fission Induced by } \\
\text { High Energy Protons }\end{array}$ & $\begin{array}{l}\text { Dubna report } \\
\text { JWNR-E-1-12902 } \\
\text { (1979)V.S.Barashenkov } \\
\text { et al }\end{array}$ & $\begin{array}{l}\text { neutron spectra, } \\
\text { nuclear cascades }\end{array}$ \\
\hline $\begin{array}{l}27 \mathrm{Al} \\
181 \mathrm{Ta}\end{array}$ & $\begin{array}{l}\text { High Energy Prown Production } \\
\text { in Medium Energy Nuclear } \\
\text { Reactions }\end{array}$ & $\begin{array}{l}\text { U of Virginia } \\
\text { Thesis(1979) } \\
\text { K.R.Cordell }\end{array}$ & \\
\hline $\mathrm{Ni}, \mathrm{Ta}$ & $\begin{array}{l}\text { Inclusive Scattering of } \\
\text { Protons on Helium, ivickel, } \\
\text { and Tantalum at } 500 \mathrm{MeV}\end{array}$ & $\begin{array}{l}\text { Phys Rev C23, } 1670 \\
\text { (1981) G. Roy } \\
\text { et al }\end{array}$ & $\begin{array}{l}\text { inclusive } \\
\text { interactions }\end{array}$ \\
\hline $\begin{array}{l}\mathrm{U}, \mathrm{Pb} \\
232 \mathrm{Th}\end{array}$ & $\begin{array}{l}\text { Neutron Production in Thick } \\
\text { Targets of Lead,Thorium and } \\
\text { Uranium Bombarded by } \\
480 \mathrm{MeV} \text { Protons }\end{array}$ & $\begin{array}{l}\text { Brookhaven report } \\
\text { BNL-NCS-51245(Vol.1) } \\
\text { p.155(1980) } \\
\text { J.S.Fraser et al }\end{array}$ & spallation \\
\hline${ }^{238} U$ & $\begin{array}{l}\text { Fission Reaction in High } \\
\text { Energy Proton Cascade }\end{array}$ & $\begin{array}{l}\text { Brookhaven report } \\
\text { BNL-NCS-51245 } \\
\text { p.133(1980) } \\
\text { H.Takahashi }\end{array}$ & nuclear cascades \\
\hline $\mathrm{Pb}$ & $\begin{array}{l}\text { Neutron Yields and Spertra } \\
\text { from } 590 \mathrm{MeV}(p, n) \text { Reactions } \\
\text { on Lead Targets }\end{array}$ & $\begin{array}{l}\text { Brookhaven report } \\
\text { BNL-NCS-51245 } \\
\text { p.201(1980) } \\
\text { S.Cierjacks et al }\end{array}$ & $\begin{array}{l}\text { neutron spectra, } \\
\text { nuclear cascades }\end{array}$ \\
\hline $235,238 \mathrm{U}$ & $\begin{array}{l}\text { Phenomerological Model for } \\
\text { Particle Production from the } \\
\text { Collisions of Nucleons and } \\
\text { Pions with Fissile Elements } \\
\text { at Medium Energies }\end{array}$ & $\begin{array}{l}\text { Clak Ridge report } \\
\text { (JRNL/TM-7528(1981) } \\
\text { F.S.Alsmiller }\end{array}$ & spallation \\
\hline $238 \mathrm{U}$ & $\begin{array}{l}\text { Spallation and Fission in } \\
\text { Heavy Metal Nuclei under Med- } \\
\text { ium Energy Proton Bombardment }\end{array}$ & $\begin{array}{l}\text { Julich Mtg. on } \\
\text { Targets for Neutron } \\
\text { Beam Spallation Sources } \\
\text { JUEL-CONF-34,p.17 } \\
\text { (1980) F.Atchison }\end{array}$ & $\begin{array}{l}\text { spallation, } \\
\text { neutron spectra }\end{array}$ \\
\hline
\end{tabular}




\begin{tabular}{|c|c|c|c|}
\hline $\begin{array}{l}40 \mathrm{Ca} \\
208 \mathrm{~Pb} \\
90 \mathrm{Zs}\end{array}$ & $\begin{array}{l}\text { Elastic Scattering of } 80-180 \\
\text { MeV Protons and the Prnton- } \\
\text { Nucleus Optical Potential }\end{array}$ & $\begin{array}{l}\text { Phys Rev C23, } 1023 \\
\text { (1981) } \\
\text { A.Nadasen }\end{array}$ & optical models \\
\hline Py & $\begin{array}{l}\text { Neuiron Production ty } \\
\text { Spallation in Heavy Metal } \\
\text { Targets: Experiments and } \\
\text { Calculation }\end{array}$ & $\begin{array}{l}\text { Julich Mtg. on } \\
\text { Targets for Neutron } \\
\text { Beam Spallation Sources } \\
\text { JUEL-CONF-34,p.(1980) } \\
\text { P.Garvey }\end{array}$ & $\begin{array}{l}\text { neutron spectra, } \\
\text { spallation }\end{array}$ \\
\hline${ }^{27} \mathrm{Al}, \mathrm{Ni}$ & $\begin{array}{l}\text { Differential Production Cross- } \\
\text { Sections of Multiply Charged } \\
\text { Fragments in } 800 \mathrm{MeV} \text { Proton- } \\
\text { Induced Spallation of Carbon, } \\
\text { Aluminum, and Nickel }\end{array}$ & $\begin{array}{l}\text { Wash. State U } \\
\text { Thesis (1978) } \\
\text { S.C.Luckstead }\end{array}$ & spallation \\
\hline $\mathrm{Ag}$ & $\begin{array}{l}\text { Fragment Production from } \mathrm{p}+\mathrm{Ag} \\
\text { Interactions at Intermediate } \\
\text { Energies }\end{array}$ & $\begin{array}{l}\text { Phys Rev C22, } 1594 \\
\text { (1980) R.E.Green, } \\
\text { R.G.Korteling }\end{array}$ & $\begin{array}{l}\text { energy spectra, } \\
\text { evaporation } \\
\text { model }\end{array}$ \\
\hline $58,62 \mathrm{Ni}$ & $\begin{array}{l}\text { Recoil Ranges of Nuclei Pro- } \\
\text { duced in the Interaction of } \\
80-164 \mathrm{MeV} \text { Protons with } \mathrm{Ni}\end{array}$ & $\begin{array}{l}\text { Phys Rev C22, } 1443 \\
\text { (1980) } \\
\text { J J astrzebski et al }\end{array}$ & $\begin{array}{l}\text { compound- } \\
\text { nucleus reaction, } \\
\text { gamma spectra, } \\
\text { nuclear cascades }\end{array}$ \\
\hline $\begin{array}{l}27 \mathrm{Al} \\
40 \mathrm{Ca}, 12 \mathrm{C}\end{array}$ & $\begin{array}{l}\text { Microscopic Description of } \\
\text { Nucleon-Nucleus Total }\end{array}$ & $\begin{array}{l}\text { Phys Rev Lett 45, } \\
527(1980)\end{array}$ & $\begin{array}{l}\text { compound-nucleus } \\
\text { reactions }\end{array}$ \\
\hline $208 \mathrm{~Pb}$ & Reaction Cross Sections & $\begin{array}{l}\text { N.J.Digiacomo } \\
\text { et al }\end{array}$ & \\
\hline $175 \mathrm{Lu}$ & $\begin{array}{l}\text { Pre-Equilibrium Process for } \\
175 \mathrm{Lu}\left(\mathrm{p}, \mathrm{xn} \text { gamma), } 176 \mathrm{Yb}\left({ }^{3} \mathrm{He},\right.\right. \\
\text { xn,yp gamma)and } 174,176 \mathrm{Yb} \\
\text { (alpha,xn gamma) Reactions }\end{array}$ & $\begin{array}{l}\text { Phys Rev C22,1002 } \\
\text { (1980) H.Sakai } \\
\text { et al }\end{array}$ & $\begin{array}{l}\text { precompound- } \\
\text { nucleus emission }\end{array}$ \\
\hline $\begin{array}{l}139 \mathrm{La} \\
159 \mathrm{~Tb}\end{array}$ & $\begin{array}{l}\text { Medium Energy Proton Induced } \\
\text { Fission in } \mathrm{Tb}, \mathrm{La} \text { and } \mathrm{Ag}\end{array}$ & $\begin{array}{l}\text { Zeit fur Physik } \\
\text { A293,241(1979) } \\
\text { G.Andersson et al }\end{array}$ & $\begin{array}{l}\text { evaporation } \\
\text { model }\end{array}$ \\
\hline${ }^{12} \mathrm{C}$ & $\begin{array}{l}\text { Do Quasifree Reaction } \\
\text { Mechanisms Explain } \\
\text { Reaction Cross Sections } \\
\text { in Intermediate-Energy } \\
\text { Proton-Nưtleus Scattering }\end{array}$ & $\begin{array}{l}\text { Phys Rev Lett 24, } \\
1579(1980) \\
\text { Y.Alexander et al }\end{array}$ & $\begin{array}{l}\text { quasi-free } \\
\text { reactions }\end{array}$ \\
\hline
\end{tabular}


Table I (Cont.)

$28 \mathrm{Si}$

$197 \mathrm{Au}$

$40 \mathrm{Ca}$

$12 \mathrm{C}$

$27 \mathrm{Al},{ }^{9} \mathrm{Be}$

$\mathrm{C}, \mathrm{Pb}$

$209 \mathrm{Bi}$

238U

$58,60 \mathrm{Ni}$

$62 \mathrm{Ni}$

$58,60 \mathrm{Ni}$
Germanium Charged Particle

Telescopes with Ultra-Thin

Detector Contacts

Use of A Ge(Li) Gamma

Detector Near A $400 \mathrm{MeV}$

Intense Proton Beam in

a p-Gamma Coincidence

Experiment

Cross-Section Measure-

ments of Nuclides

Formed by the Reaction

of $0.20-6.0 \mathrm{GeV}$

Protons with $197 \mathrm{Au}$

Scattering and Reaction

Cross Sections Measured at SPESI

Proton-Nucleus Inter-

actions at $640 \mathrm{MeV}$

Accompanied by Backward Emission of Energetic

Protons

Solid State Detectors of Fission Fragments for the Rem-Dose Measurement of Mixed and Proton and Neutron Radiation

Interaction of 80 -

$164 \mathrm{MeV}$ Protons with

Nickel Isciopes

Mechanism of Nucleon

Removal from Medium

Mass Nuclei Induced by

80 to $164 \mathrm{MeV}$ Protons

Particles and Gamma Rays

Following Preequilibrium

and Equilibrium

Reaction Processes
Nucl Inst Meth

164,121(1979)

G.S.Hubbard,

E.E.Haller

Nucl Instr Meth

165,409(1979)

C.Lebrun et al

Phys Rev C22,167

(1980)

S.B.Kaufman,

E.P.Steinberg

$\mathrm{J}$ de Physique

40,635(1979)

G.Bruge

Nucl Phys A326, 297

(1979)

V.I.Komarov et al

Health Physics $\underline{37}$,

677(1979)

M.F.Lomanov et al

Phys Rev C21,2303

(1980) R.E.Segel

Thesis(1977)

Indiana $U$

M.E.Sadler

Osaka Univ (Japan

report) RCNP-P-21

p.83 (1978)

H.Ejiri et al high-purity GE

detectors

Li-drifted $\mathrm{Ge}$ detectors, prompt gamma radiation

spallation

data compilation deuteron, alpha reactions

quasi-elastic scattering compound-nucleus reactions, gamma spectra

precompoundnucleus emission, nuclear cascades

preconipoundnucleus emission 
Table I (Cont.)

${ }^{12} \mathrm{C}$

$27 \mathrm{Al}, 40 \mathrm{C}$

${ }^{12} \mathrm{C}, 6 \mathrm{Li}$

$12 \mathrm{C}$

$12 \mathrm{C}, 6 \mathrm{Li}$

$14 \mathrm{~N}$

$238 \mathrm{U}$

$\mathrm{Br}, \mathrm{C}, \mathrm{N}$

$\mathrm{O}, \mathrm{Ag}$

$9_{\mathrm{Be}, 12 \mathrm{C}}$

${ }^{181} \mathrm{Ta}$

${ }^{238} \mathrm{U}$
Inclusive Differential

Cross Sections for

Proton Production: in?

Proton-Nucless Inter-

actions in the Model of

Coherent Fluctons

Neutron Formation from

${ }^{2} \mathrm{Li}, 10 \mathrm{~B}, 11 \mathrm{~B}, 12 \mathrm{C}$,

$160,19 \mathrm{~F}, 24,25,26 \mathrm{Mg}$,

$27 \mathrm{Al}, 40,44 \mathrm{Ca}, \mathrm{Cu}$,

$116,124 \mathrm{Sn}, 181 \mathrm{Ta}, \mathrm{Pb}$

Nuclei with $1 \mathrm{GeV}$ Protons

Proton Spectra from

$800 \mathrm{MeV}$ Protons on

Selected Nuclides

Elastic and Inelastic

Scattering of $185 \mathrm{MeV}$

Protons from $12 \mathrm{C}$

Determination of the

Optical Potential for

Elastic Proton Scat-

tering on $6 \mathrm{Li}, 12 \mathrm{C}$, and

$14 \mathrm{~N}$ at $144 \mathrm{MeV}$

Neutron Multiplication

in Uranium Bombarded

by $300-600 \mathrm{MeV}$ Protons

Scaling in Multiplicity

Distributions of Heavy,

Black and Grey Prongs in

Nuclear Emulsions

Proton Total Cross

Sections on $1 \mathrm{H}, 2 \mathrm{H},{ }^{4} \mathrm{He}$,

${ }^{9} \mathrm{Be}, \mathrm{C}$ and $\mathrm{O}$ in the Energy

Range 180 to $560 \mathrm{MeV}$

Neutron Spectrum at 900

from $800 \mathrm{MeV}(\mathrm{p}, \mathrm{n})$

Reactions on a Ta Target

Calculated Particle Pro-

duction Spectra and

Multiplicities from

Nucleon-Fissile Element

Collisions at Medium

Energies
Dubna report

JINR-R-4-12207

(1979)(Russian)

A.N.Antonov

et al

JETP Lett $\underline{30}, 86$

(1979)

V.N.Baturin

et al

Phys Rev C21,1014

(1980) R.E.Chrien

et al

Nucl Phys A319,

377(1979)

A.Ingemarsson

et al

Phys Rev C21,25

(1980) G.L.Moake,

P.T.Debevec

Sov At Energy 44 , 329(1978)

R.G.Vasil'kov et al

Physica Scripta

19,307(1979)

M.Nieminen et al

Nucl Phys A316,317

(1979) P.Schwaller

et al

Los Alamos report

LA-UR-79-3201(1979)

S.D. Howe et al

Int Conf Nuclear

Cross Sections for

Technology, Knox-

ville,CONF-791058(1979)

F.S.Alsmiller et al total cross

sections

particle production, inclusive interactions

light nuclei, knock-out reactions, quasi-elastic scattering, neutron spectra

inclusive interactions

inelastic,elastic scaltering, optical models

$i$

optical models

fission

multiplicity

neutron spectra

nuclear cascades, statistical models 


\begin{tabular}{|c|c|c|c|}
\hline $12 \mathrm{C}$ & $\begin{array}{l}\text { Inclusive Double Dif- } \\
\text { ferential Cross Sections } \\
\text { for Backward Emitted } \\
\text { Protons in Proton-Nuclei } \\
\text { Interactions at } 640 \mathrm{MeV}\end{array}$ & $\begin{array}{l}\text { Dubna report } \\
\text { JINR-E-1-11513 } \\
\text { (1978) } \\
\text { V.I.Komarov et al }\end{array}$ & energy spectra \\
\hline $\begin{array}{l}9 \mathrm{Be}, 6 \mathrm{Li} \\
181 \mathrm{Ta}\end{array}$ & $\begin{array}{l}\text { Cluster Recoil Merhanism } \\
\text { for Inclusive Nuclear } \\
\text { Reactions }\end{array}$ & $\begin{array}{l}\text { Nucl Phys A306,333 } \\
\text { (1978) R.M.Woloshyn }\end{array}$ & $\begin{array}{l}\text { inelastic } \\
\text { scattering }\end{array}$ \\
\hline $238 \mathrm{U}$ & $\begin{array}{l}\text { Analysis of Neutron } \\
\text { Yield Produced by } \\
\text { High Energy Protons }\end{array}$ & $\begin{array}{l}\text { Brookhaven report } \\
\text { BNL-26805(1979) } \\
\text { H.Takahashi, } \\
\text { Y.Nakanara }\end{array}$ & $\begin{array}{l}\text { evaporation } \\
\text { model, } \\
\text { spallation }\end{array}$ \\
\hline $\begin{array}{l}209 \mathrm{Bi} \\
197 \mathrm{Au} \\
206,207 \mathrm{~Pb} \\
208 \mathrm{~Pb}, \mathrm{Sm} \\
232 \mathrm{Th} \\
235 \mathrm{U}\end{array}$ & $\begin{array}{l}\text { Determination of Fission } \\
\text { Cross Sections of } 238 \mathrm{U} \\
235 \mathrm{U}, 232 \mathrm{Th}, 209 \mathrm{Bi}, 208 \text {, } \\
206 \mathrm{~Pb}, 197 \mathrm{Au}, 181 \mathrm{Ta} \text {, } \\
\mathrm{Yb} \text { and } \mathrm{Sm} \text { Nuclei by } \\
1 \mathrm{GeV} \text { Protons }\end{array}$ & $\begin{array}{l}\text { Yad Fiz 28,572 } \\
(1978) \\
\text { B.A.Bochagov } \\
\text { et al }\end{array}$ & \\
\hline $\begin{array}{l}27 \mathrm{Al}, 12 \mathrm{C} \\
63 \mathrm{Cu}, \mathrm{Ge} \\
56 \mathrm{Fe} \\
208 \mathrm{~Pb} \\
160\end{array}$ & $\begin{array}{l}\text { Proton-Nucleus Total } \\
\text { Cross Sections in the } \\
\text { Intermediate Energy } \\
\text { Range }\end{array}$ & $\begin{array}{l}\text { Phys Rev C20, } 1857 \\
\text { (1979) L.Ray }\end{array}$ & $\begin{array}{l}\text { total cross } \\
\text { suctions }\end{array}$ \\
\hline $\begin{array}{l}40 \mathrm{Ca} \\
56 \mathrm{Fe}, 58 \mathrm{Ni} \\
16 \mathrm{O}\end{array}$ & $\begin{array}{l}\text { Phenomenology of Proton } \\
\text { Elastic Scattering and } \\
\text { Evidence for Angular- } \\
\text { Miomentum-Dependent } \\
\text { Optical-Model Potentials }\end{array}$ & $\begin{array}{l}\text { J of Phys G5,97 } \\
\text { (1979) A.M.Kobos, } \\
\text { R.S.MacKintosh }\end{array}$ & optical models \\
\hline $\mathrm{Ag}$ & $\begin{array}{l}\text { Study of Highly Excited } \\
\text { Nuclei from } 300 \mathrm{MeV} p \\
\text { on Ag by Use of Heavy } \\
\text { Fragment Evaporation Yields }\end{array}$ & $\begin{array}{l}\text { AIP Conf Proc. } \\
47,672(1978) \\
\text { R.E.L.Green, } \\
\text { R.G.Korteling }\end{array}$ & $\begin{array}{l}\text { spallation, } \\
\text { evaporation } \\
\text { model }\end{array}$ \\
\hline \multirow[t]{2}{*}{$27 \mathrm{Al}$} & $\begin{array}{l}\text { Analysis of the Nucleons } \\
\text { Ejected in a Quasi-Elastic } \\
\text { Scattering (p,p,gamma) } \\
\text { at } 400 \mathrm{MeV}\end{array}$ & $\begin{array}{l}\text { J de Physique } 3 \text {, } \\
138(1978) \\
\text { C. Lebrun et al }\end{array}$ & $\begin{array}{l}\text { quasi-elastic } \\
\text { scattering, } \\
\text { gamma radiation }\end{array}$ \\
\hline & $\begin{array}{l}\text { Phenomenology of Nuclear } \\
\text { Inclusive Reactions }\end{array}$ & $\begin{array}{l}\text { AIP Conf Proc } 47 \text {, } \\
457(1978) \\
\text { R.M.Woloshyn }\end{array}$ & $\begin{array}{l}\text { knock-out } \\
\text { reactions, } \\
\text { inelastic } \\
\text { scattering }\end{array}$ \\
\hline
\end{tabular}




\begin{tabular}{|c|c|c|c|}
\hline $\begin{array}{l}27 \mathrm{Al}, 58 \mathrm{Ni} \\
90 \mathrm{Zr}\end{array}$ & $\begin{array}{l}\text { Medium Energy Nuclear } \\
\text { Reactions: "Quasi-Two- } \\
\text { Body Scaling" and } \\
\text { "Hot Spots" }\end{array}$ & $\begin{array}{l}\text { Phys Rev C2 } 1079 \\
\text { (1979) N.S.Wall } \\
\text { et al }\end{array}$ & $\begin{array}{l}\text { compound- } \\
\text { nucleus } \\
\text { reactions, } \\
\text { energy spectra }\end{array}$ \\
\hline \multirow[t]{2}{*}{$238 \mathrm{U}$} & $\begin{array}{l}\text { Spallation Neutron Sources } \\
\text { for Neutron Beam Research }\end{array}$ & $\begin{array}{l}\text { Contemporary Phys } \\
\underline{19}, 505(1978) \\
\text { G.Manning }\end{array}$ & spallation \\
\hline & $\begin{array}{l}\text { Measurements and Calcula- } \\
\text { tions of Reaction Losses } \\
\text { of Medium-Energy Protons } \\
\text { in NAI Detectors }\end{array}$ & $\begin{array}{l}\text { Nucl Instr Meth } \\
153,511(1978) \\
\text { C.A.Goulding, } \\
\text { J.G.Rogers }\end{array}$ & $\begin{array}{l}\text { NAI detecturs, } \\
\text { energy losses }\end{array}$ \\
\hline $\begin{array}{l}\text { Al,Fe } \\
238 \mathrm{U} \\
\text { Lucite }\end{array}$ & $\begin{array}{l}\text { Thick-Target Neutron and } \\
\text { Gamma Ray Yields Induced } \\
\text { by Medium-Energy Proton } \\
\text { and Deuteron Bombardments }\end{array}$ & $\begin{array}{l}\text { Lawrence Livermore } \\
\text { Nat Lab report } \\
\text { UCID-18086(1979) } \\
\text { J.A.Becker et al }\end{array}$ & gamma radiation \\
\hline \multirow[t]{2}{*}{$\begin{array}{l}232 \mathrm{Th} \\
238 \mathrm{U}\end{array}$} & $\begin{array}{l}\text { Breeding Nuclear Fuel With } \\
\text { Accelerators: The Electro- } \\
\text { nuclear Method. Nuclear } \\
\text { Physics Aspects }\end{array}$ & $\begin{array}{l}\text { Sov J Particles \& } \\
\text { Nuciei(U.S.) } 9452 \\
\text { V.S.Barashenkov (1978) }\end{array}$ & \\
\hline & $\begin{array}{l}\text { Analysis of Nuclear Fissil- } \\
\text { ity for Intermediate- } \\
\text { Energy Ptoton Induced } \\
\text { Reactions }\end{array}$ & $\begin{array}{l}\text { Zeit fur Physik } \\
\text { A287,37(1978) } \\
\text { A.S.Ilinov et al }\end{array}$ & $\begin{array}{l}\text { fission, } \\
\text { evaporation, } \\
\text { precompound- } \\
\text { nucleus emission }\end{array}$ \\
\hline $\mathrm{Yb}$ & $\begin{array}{l}\text { Observation of the Effect } \\
\text { of Central Collisions } \\
\text { Leading to Formation of } \\
\text { a Compound Nucleus in } \\
\text { Interaction of } 200-\mathrm{MeV} \\
\text { Protons with Nuclei }\end{array}$ & $\begin{array}{l}\text { Sov J Nucl Phys } \\
27,751(1978) \\
\text { O.E.Shigaev et al }\end{array}$ & $\begin{array}{l}\text { heavy nuclei, } \\
\text { compound nuclei }\end{array}$ \\
\hline $\begin{array}{l}208 \mathrm{~Pb} \\
58 \mathrm{Ni} \\
120 \mathrm{Sn} \\
90 \mathrm{Zr}\end{array}$ & $\begin{array}{l}\text { Elastic Scattering of } 100 \\
\text { MeV Protons and Systematic } \\
\text { Optical Model Analysis }\end{array}$ & $\begin{array}{l}\text { Nucl Phys A301, } \\
349 \text { (1978) } \\
\text { K.Kwiatkowski, } \\
\text { N.S.Wall }\end{array}$ & optical models \\
\hline $\begin{array}{l}40,42 \mathrm{Ca} \\
12 \mathrm{C}, 209 \mathrm{~Pb} \\
58 \mathrm{Ni}\end{array}$ & $\begin{array}{l}\text { Microscopic Approaches to } \\
\text { the Intermediate Energy } \\
\text { Nucleon-Nucleus Optical } \\
\text { Potential }\end{array}$ & $\begin{array}{l}\text { U of Texas } \\
\text { Thesis(1977) } \\
\text { R.L.Ray }\end{array}$ & optical models \\
\hline $\mathrm{Sm}, \mathrm{Yb}$ & $\begin{array}{l}\text { L-Shell X-Ray Production } \\
\text { Cross Sections Due to } 1 \mathrm{H} \text {, } \\
{ }^{4} \mathrm{He} \text {, and } 7_{\mathrm{Li}} \text { Ion Bombard- } \\
\text { ment of Selected Thin Rare } \\
\text { Earth and } \mathrm{Pb} \text { Targets }\end{array}$ & $\begin{array}{l}\text { North Texas State } \\
\text { U, Thesis(1978) } \\
\text { G.M.Light }\end{array}$ & $x$-radiation \\
\hline
\end{tabular}


Table I (Cont.)

$27 \mathrm{Al}, 12 \mathrm{C}$

$\mathrm{Ni}$

$27 \mathrm{Al},{ }^{40} \mathrm{Ca}$

$12 \mathrm{C}, 6 \mathrm{Li}$

$7 \mathrm{Li}, 24 \mathrm{Mg}$

${ }^{209} \mathrm{Bi}$

$238 \mathrm{U}$

$27 \mathrm{Al}, \mathrm{Cu}$

Pb.Ti,W

$\mathrm{U}$

$\mathrm{Ca}, 208 \mathrm{~Pb}$

$\mathrm{Si}, 90 \mathrm{Zr}$

$27 \mathrm{Al},{ }^{9} \mathrm{Be}$

10.11B,

$12 \mathrm{C}$

$\mathrm{C}, 6 \mathrm{Li}$

$181 \mathrm{Ta}$
Analysis of Neutron Yield

Produced thy High-Energy

Protons

Differential Production

Cross Sections of Míultiply

Charged Fragments in

$800 \mathrm{MeV}$ Proton-Iriduced

Spallation of Carbon,

Aluminum, and Nickel

Low-Energy Particles in

Nuclear Reactions Pruduced

by Nuclei with Energies

of $0.1 .1 \mathrm{GeV} / \mathrm{Nucleon}$

Quasielastic Knockout of

Alpha Clusters from

Light and Medium Nuclei

by $600 \mathrm{MeV}$ Protons

Nucleon Losses by Uranium and Bismuth Nuclei at HighEnergy Fission

Systematics of 00 Neutron

Production by $800 \mathrm{MeV}$

Protons on Targets with

$27<A<238$

Elastic Scattering of 80-160

$\mathrm{MeV}$ Protons from $208 \mathrm{~Pb}$,

$90 \mathrm{Zr}, \mathrm{Ca}$ and Si Targets

Backward-Pick-Up Reactions

for Inclusive Spectra of

Energetic Deuterons at $180^{0}$

in Proton-Nucleus Collisions

Inelastic Scattering of

High-Energy Particles

from Light Nuclei and

Cluster Structure of Nuclei

1800 Production of Protons

in High-Energy p, d, and

Alpha Reactions in Nuclei
Trans Am Nucl Soc

30,739(1978)

Y.Nakahara,

H.Takahashi

Los Alamos repnrt

LA-7464-T(1978)

Thesis

S.C.Luckstead

Sov J Nucl Phys

27, 69(1978)

S.D.Bogdanov

V.I.Ostroumov

Phys Rev C18,

1776 (1978)

G.Landaud et a!

JETP Lett 27,309

(1978) V.E.Shashmin

Phys Rev C18, 1418

(1978) B.E.Bonner

et al

Indiana $U$

Thesis(1977)

ANadasen

Phys Lett B72,16

(1977) T.Fujita

Yad Fiz 25,3(1977)

V.V.Avdeichikov

et al

Phys Lett B71,79

(1977) H.Brody

et al fission

evaporation

models,

spallation

spallation, evaporation model

nuclear emulsions

knock-out reactions

charge exchange reactions, neutron spectra

optical models

backscattering, pickup reaction

spallation 
Table I (Cont.)

$$
{ }^{40} \mathrm{Ca},{ }^{12} \mathrm{C}
$$

${ }^{27} \mathrm{Al},{ }^{9} \mathrm{Be}$

$12 \mathrm{C}, \mathrm{Pb}$

27 Al

197 Au,Ag

$181 \mathrm{Ta}$

160

${ }^{59} \mathrm{Co}, 56 \mathrm{Fe}$

$58 \mathrm{Ni}$

${ }^{9} \mathrm{Be}$
Determination of the Pene-

tration Range with the Aid

of Total Cross Sections of

Protons, Pions, and Alpha

Particles Between $200 \mathrm{MeV}$

and $2 \mathrm{GeV}$

Inclusive Spectra and the

Angular Distribution of

Protons Emitted Backwards

in the Interaction of 640

$\mathrm{MeV}$ Ptotons with Nuclei

High Energy (E less than or equal to $1000 \mathrm{GeV}$ ) Intranuclear Cascade Model for Nucleons and Pions Incident on Nuclei and Comparisons with Experimental Data

Cross Section Measurements of High Energy Proton Induced Reactions in Gold, Tantalum and Silver Targets

"Correlated Clusters" and Inclusive Spectra of Energetic Protons at $180^{\circ}$ in Proton-Nucleus Collisions

Neutron Spectra at 00 Degrees from 83.7-MeV Deuterons and 100.2-MeV Protons on Beryllium

Maxwell Distribution and Mass Motion in p-Nucleus Reactions

Gamma Spectrometry with High Energy Protons

Systematics in the Interaction of IntermediateEnergy Protons with Medium-Mass Nuclei

Calculation of the Nuclear Reaction Cross Sections with an Intranuclear-

Cascade Model
J Plasma Phys $\underline{3}$, 1497(1977)

I.Brissaud

Phys Lett B69.37

(1977) V.I.Komarov

et al

Oak Ridge report

ORNL/TM-5710(1977)

H.W.Bertini et al

nuclear cascades
Incrg \& Nucl Chem Lett 13,205(1977)

S.Kraemer et al

Phys Rev Lett 39 , 174(1977) T.Fujita

Medical Physics 4 322(1977) R.Madey et al

Phys Rev D15,2533 (1977) T.F.Hoang

Saclay report CEA-N-1861(nd) J.P.Alard et al

Phys Rev Lett 17 , 950(1977) M.Sadler et al

Japan Atomic Research Institute report JAERI-M-6306 (1975) S.Baba inclusive interactions

total cross

sections inclusive interactions, backscattering

neutron spectra inelastic scattering, Bolzmann statistics

prompt gamma radiation

precompoundnucleus, gamma spectra

heavy nuclei, Monte Carlo method, nuclear cascades 
27 $\mathrm{Al}, 9 \mathrm{Be}$
$\mathrm{C}, \mathrm{Pb}$
$9 \mathrm{Be}^{12 \mathrm{C}}$
$27 \mathrm{Al}$

${ }^{9} \mathrm{Be}, \mathrm{C}, \mathrm{Li}$

${ }^{9} \mathrm{Be}, 12 \mathrm{C}$

$27 \mathrm{AJ}$

Ti,S1V,Fe

$6 \mathrm{Li}$

$90 \mathrm{Zs}$
Protnn and Deuteron Double

Differential Cross Sections

at Angles from $10 \mathrm{Deg}$ to 60

Deg from $\mathrm{De}, \mathrm{C}, \mathrm{Al}, \mathrm{Fe}, \mathrm{Cu}$,

$\mathrm{Ge}, \mathrm{W}$, and $\mathrm{Pb}$ Under S58-MeV.

Proton Irradiation

Neutron Spectra at Zero

Degrees from Proton Bombard-

ment of Light Nuclei at

Medium Energies

Production of Fast Fragments by Bombarding Light Nuclei with Intermediate-Energy

Protons

Neutron Spectra at 00 from $(p, n)$ Reactions on ${ }^{9} \mathrm{Be}, 12 \mathrm{C}$, and $27 \mathrm{Al}$ at 647 and $800 \mathrm{MeV}$

A Two-Parameter Scintillation Spectrometer System for Measurement of Secondary $p, d, t$ Distributions from Materials under 558 MeV Proton Irradiation

Recoil Propteries of Spallation Products from Bombardment of $Z=13-29$ Targets with 150, 300, and $600 \mathrm{MeV}$ Protons

Elastic and Inelastic Scattering of $185 \mathrm{MeV}$ Protons from $6 \mathrm{Li}$

Monte-Carlo Evaporation Calculations for the Interpretation of Isotope Yield Distribution in High Energy Nuclear Reactions
NASA report NASA-

TN-D-8119(1976)

SM.Beck,

C.A.Powell

Univ of New Mexico

Thesis(1975)

C.G.Cassapakis

Nucl Phys A256,362

(1976) V.I.Komarov

et al

Phys Lett B63,35

(1976)

C.G.Cassapakis et al

NASA report NASA-

TN-D-7925(1975)

S.M.Beck

Nucl Phys A260,

369(1976) M.Lagarde-

Simonoff et al

Zeit fur Physik

A275, 169(1975)

M.Y.M.Hassan et al

Marburg U,Germany

Thesis(1974)

G.Stark proton reactions, quasi-elastic

scattering

charge-exchange reactions

quasi-elastic scattering

charge-exchange reactions, neutron spectra

time of flight spectrometer

spallation

impulse approximation

heavy nuclei, intermediate mass nuclei, evaporation model, MonteCarlo method, Weisskopf Model

Carnegie-Mellon U Thesis(1976)

knock-out reactions
Systematic Sudy of ProtonReactions 


\begin{tabular}{|c|c|c|c|}
\hline $\begin{array}{l}27 \mathrm{Al}, \mathrm{C} \\
\mathrm{Cu}, \mathrm{Fe}\end{array}$ & $\begin{array}{l}\text { Interlaboratory Comparison } \\
\text { of Spallation-Reaction } \\
\text { Cross Sections for Iron and } \\
\text { Copper with } 590-\mathrm{MeV} \text { Protons }\end{array}$ & $\begin{array}{l}\text { J Inorg \& Nucl } \\
\text { Chem 38, 13(1976) } \\
\text { C.J. Orth et al }\end{array}$ & spallation \\
\hline $\begin{array}{l}\text { U } \\
\text { gypsum }\end{array}$ & $\begin{array}{l}\text { Spectrum and Shielding } \\
\text { Measurements and Calcu- } \\
\text { lations of Neutrons } \\
\text { Produced by } 800 \mathrm{MeV} \\
\text { Protons }\end{array}$ & $\begin{array}{l}\text { Proc on Nucl Cross } \\
\text { Sections \& Tech, } \\
\text { Washington,DC,NBS- } \\
\text { Spec Pub } 425,476 \\
\text { (1975) L.R.Veeser et al }\end{array}$ & $\begin{array}{l}\text { neutron } \\
\text { emission }\end{array}$ \\
\hline $\begin{array}{l}27 \mathrm{Al}, \mathrm{Mo} \\
\mathrm{La}, \mathrm{V}\end{array}$ & $\begin{array}{l}\text { Spallation Cross Sections } \\
\text { and the LAMPF Medical } \\
\text { Radioisotope Program }\end{array}$ & $\begin{array}{l}\text { NBS Spec Pub } 425 \\
492(1975) \text { B.R.Erdal } \\
\text { et al }\end{array}$ & spallation \\
\hline $\begin{array}{l}{ }^{40} \mathrm{Ca},{ }^{208}{ }_{\mathrm{rb}} \\
20 \mathrm{Ne}, 58 \mathrm{Ni} \\
{ }^{154} \mathrm{Sm}\end{array}$ & $\begin{array}{l}\text { Effective Nucleon-Nucleon } \\
\text { Forces and Description of } \\
\text { Continuous and Discrete } \\
\text { Spectra in Semimicroscopic } \\
\text { Approach }\end{array}$ & $\begin{array}{l}\text { 34th Conf on Nucl } \\
\text { Spect \& Nucl } \\
\text { Struct..Alma-Ata, } \\
\text { USSR,INIS-SU-282 } \\
\text { p.424(1984) } \\
\text { O.M.Knyaz'kov et al }\end{array}$ & prui...us.tetion \\
\hline $120 \mathrm{Sn}$ & $\begin{array}{l}\text { Analysis of a MuItistage } \\
\text { Mechanism of the (p,p') } \\
\text { Reaction in the Quasi } \\
\text { Particle-Phonon Model of } \\
\text { a Preequilibrium Decay }\end{array}$ & $\begin{array}{l}\text { 34th Conf on Nucl } \\
\text { Spect \& Nucl } \\
\text { Struct.Alma-Ata, } \\
\text { USSR,INIS-SU-282, } \\
\text { p.302(1984)A.K.Deb et al }\end{array}$ & $\begin{array}{l}\text { precompound- } \\
\text { nucleus emission }\end{array}$ \\
\hline $56 \mathrm{Fe}, 60 \mathrm{Ni}$ & $\begin{array}{l}\text { Energy Spectra of Particles } \\
\text { with } \mathrm{Z}<2 \text { From The } 60 \mathrm{Ni} \text {, } \\
62 \mathrm{Cu} \text { and } 64 \mathrm{Zn} \text { Intermediate } \\
\text { System Decay }\end{array}$ & $\begin{array}{l}\text { Bull Acad of } \\
\text { Sciences,Phys. } \\
\text { Series } 48,1006 \\
\text { (1984)N.T.Burtebaev et al }\end{array}$ & $\begin{array}{l}\text { precompound- } \\
\text { nucleus emission, } \\
\text { energy spectra }\end{array}$ \\
\hline $54 \mathrm{Fe}, 58 \mathrm{Ni}$ & $\begin{array}{l}\text { Effect of the First Stage } \\
\text { of Multistep Direct Pro- } \\
\text { cesses at the Energy } \\
\text { of the Order of } 100 \mathrm{MeV}\end{array}$ & $\begin{array}{l}\text { Bull Acad of } \\
\text { Sciences,Phys. } \\
\text { Series 47,2224 } \\
\text { (1983)F.A.Zhivo- } \\
\text { pistsev et al }\end{array}$ & $\begin{array}{l}\text { direct reactions, } \\
\text { precoinpound- } \\
\text { nucleus emission }\end{array}$ \\
\hline $6.7 \mathrm{Li}$ & $\begin{array}{l}\text { Energy Spectra and Angular } \\
\text { Correlation Functions of } \\
\text { Charged Particles from the } \\
6 \mathrm{Li}(\mathrm{p}, \mathrm{pd}) \text { and } 7 \mathrm{Li}(\mathrm{p}, \mathrm{pt}) \text { at } \\
\text { the lncident Proton Energy } \\
\text { of } 70 \mathrm{MeV}\end{array}$ & $\begin{array}{l}\text { 34th Conf on Nucl } \\
\text { Spect \& Nucl } \\
\text { Struct,Alma-Ata, } \\
\text { USSR,INIS-SU-282 } \\
\text { p.290(1984) } \\
\text { M.V.Pasechnik et al }\end{array}$ & $\begin{array}{l}\text { quasi-elastic } \\
\text { scattering, } \\
\text { proton spectra }\end{array}$ \\
\hline $\begin{array}{l}{ }^{59} \mathrm{Co} \\
56,57 \mathrm{Fe}\end{array}$ & $\begin{array}{l}\text { Proton Pre-Equilibrium } \\
\text { Spectra from Decay of the } \\
\text { Composite System } 60 \mathrm{Ni}\end{array}$ & $\begin{array}{l}\text { 3rd Int Symp on } \\
\text { Neutron Induced } \\
\text { Reactions,p.335 } \\
\text { (1982) Smolenice, } \\
\text { Czech. A.D.Duisebaev } \\
\text { et al }\end{array}$ & $\begin{array}{l}\text { precompound- } \\
\text { nucleus emission }\end{array}$ \\
\hline
\end{tabular}


${ }^{59} \mathrm{Co}$

${ }^{9} \mathrm{Be}$

${ }^{12} \mathrm{C}, 24 \mathrm{Mg}$

$28 \mathrm{Si}$

$152 \mathrm{Sm}$

$6 \mathrm{Li}$

9Be

$T h, 233 \mathrm{U}$

$209 \mathrm{Bi}$

${ }^{54} \mathrm{Fe}, 12 \cup \mathrm{Sn}$

i $18 \mathrm{Sn}, 90 \mathrm{Zr}$
Tairing and Shell

Effects in $(p, x n)$ Preequilibrium Emission

One- and Two- Neutron

Pick-Up Reactions Induced by Protons Interacting with 9 Be

Inelastic Scattering of 65

$\mathrm{MeV}$ Protons from ${ }^{12} \mathrm{C}, 24 \mathrm{Mg}$. and $28 \mathrm{Si}$, and $32 \mathrm{~S}$

Levels in 149Eu from the $(\mathrm{p}, 4 \mathrm{n}$ gamma) Reaction

Evidence for Multistep

Processes in Proton

Capture Reactions

Reaction of (p,pn) on

Lithium Isotopes at the

Proton Energy of $70 \mathrm{MeV}$

One-Neutron and Two-Neutron

Pickup in Interaction of

Protons with ${ }^{9} \mathrm{Be}$

Linear Momentum Transfer in Intermediate-Energy

Projectile-Nucleus

Collisions

Contribution of One- and

$\mathrm{Tr}$ ᄀ- Step Processes to

Inclusive (p,d) Reactions

Mechanism of the (p,alpha)

Reaction: Pick-Up or Knock-

Out
Workshop on Coinci-

dent Particle

Emission from

Continuum States,

Bad Honnef(W.Ger-

many)INIS-MF-9280

(1984)M.Blann

et al

Yad Fiz 39, 1081

(1984) N.l.Zaika

et al

Phys Rev C31,1616

(1985) S.Katoic

et al

Nuovo Cimento

A82,253(1984)

G. Lo Bianco et al

Phys Rev C31,660

(1985) H.J.Hausman

34th Conf on $\mathrm{Nucl}$

Spect \& Nucl

Struct,Alma-Ata,

USSR,INIS-SU-282, p.282(1984)

O.K.Gorpinich et al

Yad Fiz 39,682

(1984)N.I.Zaika

et al

3rd Winter Workshop on Nuclear Dynamics, Copper Min.,Co.

CONF-8403101 pp.126-

130(1984)K.Kwiatkowski

et al

Nucl Phys A424, 1

(1984) A.O.Gattone

et al

Zeit fur Physik

A318, 145(1984)

E.Gadioli et al intermediate mass nuclei, precompoundnucleus emission. inclusive interactions, neutron spectra

pickup reactions

elastic and inelastic scattering

electron spectra, gamma spectra, $\mathrm{k}$ conversion

capture

energy spectra

one-nucleon transfer reactions, pickup reactinns

nuclear cascades

deuteron spectra, inclusive interactions

knock-out reactions 
$27 \mathrm{Al}, 58 \mathrm{Ni}$

$93 \mathrm{Nb}$

${ }^{209} \mathrm{Bi}$

$197_{\mathrm{Au}}$

$90 \mathrm{Zr}$

$116,118 \mathrm{Sn}$

$122 \mathrm{Sn}$

$12 \mathrm{C}$

160

9Be

$48,50 \mathrm{Ti}$

$\mathrm{C}, \mathrm{Cu}, \mathrm{Pb}$
"One, Two, Infinity" Model for Precompound Reaction and Nuclear Size Effect

Analysing Power of Neutron Continuum Spectra for the 93Nb(p,nx) Reaction at 65 $\mathrm{MeV}$

Extension of this Generalized Exciton Model and Calculations of (p,p') and (p,alpha) Angular Distributions

DWBA Description of the $90 \mathrm{Zr}$ (p,d) $89 \mathrm{Zr}$ Reaction at Energies from $20 \mathrm{MeV}$ to $185 \mathrm{MeV}$

Analysis of (p,p), (p,n), and $(n, n)$ Scattering on the Even Tin Isotopes Using the Lane Coupled Equations

Intranuclear Cascade and Fermi Breakup Calculations of $1 \mathrm{H}$ - and $4 \mathrm{He}$-Induced Reactions On Light Target Nuclei

Comparison of the Neutron Fields Produced by $50 \mathrm{MeV}$ Deuterons and $65 \mathrm{MeV}$ Protons on ${ }^{9} \mathrm{Be}$ at the Louvain-LaNeuve Cyclotron

$48 \mathrm{Ti}$ and $50 \mathrm{Ti}$ (p,xpyn) Excitation Functions Induced by Protons from 5-85 $\mathrm{Me} \curlyvee$. Comparison with Pre-Equilibrium Calculations

Neutron and Photon Production from Thick Targets Bombarded by $30-\mathrm{MeV}$ p, 33-MeV d, 65-MeV 3He, and $65 \cdot \mathrm{MeV}$ Alpha Ions: Phenomenological Analysis of Experimental Neutron Energy Spectra
Chinese Physics 4 , 636(1984)

Ji-Zhi Pan, Huan-

Cheng Chiang

J De Physique

(Paris)45,31(1983)

H.Sakai et al

Nucl Phys A419,472

(1984) A.Iwamoto,

K.harada

Phys Lett B137,334

(1984) P.W.F.Alons

et al

Phys Rev C29, 1710

(1984) C. Wong et al

Phys Rev C29, 1606 (1984) A.Goekmen

et al

Int Conf Nuclear

Data for Sci \& Tech

Antwerp,Belgium

Sept 6-10(1982)

J.P.Meulders et al

McGill Univ, Montreal, Canada

Thesis(1979)

K.I.W.Burns

Phys Rev C29, 1317 (1984) T.Nakamura, Y.Uwamino precompoundnucleus, neutron spectra

inelastic scattering, neutron spectra

inelastic scattering, pickup reactions, precompoundnucleus emission

proton-neutron interactions, one-nucleon transfer reactions

charge-exchange reactions

breakup reactions, nuclear cascades

neutron sources

quasi-elastic scattering

stripping, breakup reaction, evaporation model, nuclear cascades, precompoundnucleus emission, Serber Theory 


\begin{tabular}{|c|c|c|}
\hline $\begin{array}{l}\mathrm{C}, \mathrm{Cu}, \mathrm{Fe} \\
\mathrm{Pb}\end{array}$ & $\begin{array}{l}\text { Neutron and Photon Produc- } \\
\text { tion from Thick Targets } \\
\text { Bombarded by 30-MeV p, } \\
\text { 33-MeV d, 65-MeV } 3 \mathrm{He} \text {, and } 65- \\
\text { MeV Alpha Ions: Experiment } \\
\text { and Comparison with Cascade } \\
\text { Monte Carlo Calculations }\end{array}$ & $\begin{array}{l}\text { Phys Rev C29,1307 } \\
\text { (1984) K.Shin et al }\end{array}$ \\
\hline $\begin{array}{l}46,48 \mathrm{Ti} \\
50 \mathrm{Ti}\end{array}$ & $\begin{array}{l}\text { Elastic and Inelastic Scat- } \\
\text { tering of } 100 \mathrm{MeV} \text { Protons } \\
\text { from the Even-Even Titanium } \\
\text { Isotopes }\end{array}$ & $\begin{array}{l}\text { Phys Rev C29,794 } \\
\text { (1984) L.W.Woo et } \\
\text { al }\end{array}$ \\
\hline $14 \mathrm{~N}$ & $\begin{array}{l}\text { (p,n) Reactions on }{ }^{14} \mathrm{C} \\
\text { and } 14 \mathrm{~N} \text { and the Effective } \\
\text { Nucleon-Nucleon Interaction }\end{array}$ & $\begin{array}{l}\text { Phys Rev C29,764 } \\
\text { (1984) } \\
\text { T.N.Taddeucci } \\
\text { et al }\end{array}$ \\
\hline $\mathrm{Ge}$ & $\begin{array}{l}\text { Excitation Functions of } \\
\mathrm{Ge}(\mathrm{p}, \mathrm{xnyp}) \text { Reactions and } \\
\text { Production of } 68 \mathrm{Ge}\end{array}$ & $\begin{array}{l}\text { Int J of Appl Rad } \\
\text { \& Isotopes } 34,1531 \\
\text { (1983) T.Horiguchi } \\
\text { et al }\end{array}$ \\
\hline
\end{tabular}

58 $\quad$ Two-Step Description of a (p, ${ }^{3}$ He $x$ )-Reaction to the Continuum

$6,7_{\mathrm{Li}}$

(p,px) Reaction on $6_{\mathrm{Li}}$ and

7 $\mathrm{Li}$ Nuclei at Incident

Proton Energy of $70 \mathrm{MeV}$

$\begin{array}{ll}9 \mathrm{Be} & \begin{array}{l}\text { Neutrons Produced by the } \\ \text { p + Be and the } d+ \\ \text { Be Reactions, and the } \\ \text { Cross Section Measurement } \\ \text { with these Neutrons }\end{array} \\ 208 \mathrm{~Pb}, 7 \mathrm{Li} & \begin{array}{l}\text { Total Neutron Yields from } \\ 100 \mathrm{MeV} \text { Protons on } \mathrm{Pb} \\ \text { and } 7 \mathrm{Li} \text { Targets }\end{array} \\ 209 \mathrm{Bi}, 12 \mathrm{C} & \begin{array}{l}\text { Edge and Clusier Effects } \\ \text { in Precompound Nuclear } \\ \text { Reactions }\end{array}\end{array}$

Hel Phys Acta 56, 975(1983)

$\mathrm{H}$ Jasicek et al

Bull Acad Sciences Phys Series 47, 185(1983)

O.K.Gorpinich

Meet on Heavy and Light Ion Irrad. \&

Rad. Measurement Tokyo,Japan p.33 (1981) K.Sugiyama

Nucl Instr \& Meth 214,333(1983)

M.A.Lone et al

Zhonghua Heyixue

Zazhi(China) 4,216

(1982) W.Ye ei al nuclear cascades, neutron spectra

elastic

scattering, optical models

charge-exchange nucleon-nucleon interaction, optical models

knock-out reactions, quasi-elastic scattering, nuclear reaction yield

two-nucleon transfer

quasi-free reactions

neutron spectra

knock-out reactions

precompound nucleus emission, nuclear cascades 


\section{Table T (Cont.)}

$27 \mathrm{Al}$

$233,235 U$

$27 \mathrm{Al}, 209 \mathrm{Bi}$

${ }^{79} \mathrm{Br},{ }^{197} \mathrm{Au}$

$65 \mathrm{Cu}, 89 \mathrm{Y}$

$64 \mathrm{Zn}$

197 AL:

$202 \mathrm{Hg}$

$40 \mathrm{Ca}, 208 \mathrm{~Pb}$

$9_{\mathrm{Be}}$

$27 \mathrm{Al}, 59 \mathrm{Co}$

$27 \mathrm{Al}, 59 \mathrm{Co}$

$197 \mathrm{Au}, 232 \mathrm{Th}$
Emission of Alpha Particles

in Statistical Multistep

Compound Reactions

Pre-Equilibrium Emission of Light Composite Particles

in the Framework of the

Exciton Model

Isotopic and Neutron Yields of Products from the Asymmetric and Near-Symmetric Proton-Induced Fission of 233 $\mathrm{U}$ and $235 \mathrm{U}$

Cascade-Exciton Model of Nuclear Reactions

Double Differential Cross Sections for $(p, x n)$ Reactions of $64 \mathrm{Zn}, 65 \mathrm{Cu}$ and $89 \mathrm{Y}$ with $26 \mathrm{MeV}$ Protons

Pre-Equilibrium Decay of Nuclei with A Approximately Equal 200 at Excitation Energies to $90 \mathrm{MeV}$

"Wine-Bottle" Optical Potentials and the Inelastic Scattering of Protons Near $200 \mathrm{MeV}$

Fragmentation Spectra Induced by Light Ions Colliding With Light Nuclei

Interpretation of Some $(p, n),(n, p)$, and $\left({ }^{3} \mathrm{He}, \mathrm{p}\right)$ Reactions by Means of the Statisticai Multistep Compor : id Emission Theory

Proton-Induced Alpha -and Tau-Emission at $72 \mathrm{MeV}$
Phys Rev C28, 1892 (1983) R.Bonetti

et al

Phys Rev C28, 1527 (1983) K.Sato et al

Phys Rev C28, 1188 (1983) P.A.Beeley

et al

Nucl Phys A401,329 (1983) K.K.Gudima et al

Zeit fur Physik A311,323(1983)

W.Scobel et al

Nuovo Cimento A40, 383(1977) E.Gadioli et al

Nucl Phys A394,349 (1983) G.R.Satchler

Phys Rev C27, 1876 (1983) T.Delbar et al

Phys Rev C27, 1003 (1983) R.Bonetii et al

Nuovo Cimento Lett 28.15(1980)

Z.Lewandoski et al statistical

models

precompound-

nucleus emission

fission yield

inclusive interactions, charge-exchange reactions

neutron spectra

precompoundnucleus emission

inelastic scattering, optical models

fragmentation, inclusive interactions, quasi-free reactions

charge-exchange reactions, statistical models

emission spectra 
Table I (Cont.) $\begin{array}{ll}27 \mathrm{Al}, 59 \mathrm{Co} & \begin{array}{l}\text { Semierspirical Calculation } \\ \text { of Excitation Functions }\end{array}\end{array}$

$32 \mathrm{~S}$

$\mathrm{Be}$

$\mathrm{Pb}, \mathrm{Li}$

$209 \mathrm{Bi}$

$208 \mathrm{~Pb}$

$27 \mathrm{Al}, 209 \mathrm{Bi}$

$24 \mathrm{Mg}$

$120 \mathrm{Sn}$

$3 \mathrm{He}, 238 \mathrm{U}$
Energy Dependence of

Optical-Model Parameters

for the $p+32 S$ System

between 15 and $135 \mathrm{MeV}$

Comparison of Neutron

Therapy Beams Produced by

$50 \mathrm{MeV}$ Deuterons and 65

MeV Protons on Beryllium

Measured and Calculated

Neutron Yields for

$100 \mathrm{MeV}$ Protons on Thick

Targets of $\mathrm{Pb}$ and $\mathrm{Li}$

Analysis of ( $\left.p, p^{\prime}\right)$ Reac-

tions into the Continuum

in the Microscopic Moral

Continuum Spectra in

Light-Ion Reactions

Elastic and Inelastic

Scattering of Protons

from $24 \mathrm{Mg}$

Statistical Multistep

Direct Emission Theory

Revisited

Pion Production and

Charged-Particle

Multiplicity Selection

in Relativistic Nuclear

Collision
Int Conf on $\mathrm{Nucl}$

Cross Sections for

Tech.Knoxville, NBS

Spec Pub 594,778(1979)

E.L.Petersen

Nuovo Cimento

A59, 101(1980)

R.De Leo et al

Acta Radiol Oncology,

Rad. Therapy, Physics

\& Biology 21,281(1982)

S.Vynckier et al

Proc 6th Meeting Int

Collaboration of

Adv.Neutron Sources

June 28, 1982

Argonne report ANL-

82-80 p.583, R.T.Jones

et al

INIS-MF-7523(1981)

Annual Report

Texas U, Austin

T.Tamura, H.Lenske

Int Symp on Highly

Excited States in

Nuclear Reactions

Osaka,Japan, May 1980

H.Ikegami,M.Muraoka

Phys Rev C27,482

(1983)D.K.Hasell et al

Phys Leti B112,189

(1982) M.S.Hussein,

R.Bonneti

Phys Rev C26, 2572

(1982)K.L.Wolf

et al excitation

functions

optical models

neutron beams

spallation, neutron emission

inelastic scattering, particle-hole model

direct

reactions

optical models

direct reactions, cascade theory, statistical models

proton reactions, inclusive interactions 


\begin{tabular}{|c|c|c|c|}
\hline $54 \mathrm{Fe}, 120 \mathrm{Sn}$ & $\begin{array}{l}\text { Mechanism of Cluster } \\
\text { Emission in Nucleon-Induced } \\
\text { Preequilibrium Reactions }\end{array}$ & $\begin{array}{l}\text { Phys Rev C26, } 1821 \\
\text { (1982) A.Iwamoto, } \\
\text { K.Harada }\end{array}$ & $\begin{array}{l}\text { compound-nucleus } \\
\text { emission }\end{array}$ \\
\hline & $\begin{array}{l}\text { Study of the Hard Part } \\
\text { of the Neutron Spectra } \\
\text { in }(p, n) \text { Reactions }\end{array}$ & $\begin{array}{l}\text { Sov J of Nucl Phys } \\
35,474(1982) \\
\text { N.S.Biryukov et al }\end{array}$ & $\begin{array}{l}\text { precompound- } \\
\text { nucleus emission }\end{array}$ \\
\hline \multirow[t]{2}{*}{$208 \mathrm{~Pb}$} & $\begin{array}{l}\text { Quasiparticle-Phonon Model } \\
\text { of the Preequilibrium } \\
\text { Decay }\end{array}$ & $\begin{array}{l}\text { INIS-SU-125 p.440 } \\
\text { 32. Conf Nucl Spec- } \\
\text { troscopy \& Nucl } \\
\text { Structure, Kiev } \\
\text { (1982)F.A.Zhivo- } \\
\text { pistsev et al }\end{array}$ & $\begin{array}{l}\text { precompound- } \\
\text { nucleus emission, } \\
\text { inelastic } \\
\text { scattering }\end{array}$ \\
\hline & $\begin{array}{l}\text { Study on the High Energy } \\
\text { Range of the Spectra } \\
\text { in the }(p, n) \text { Reaction }\end{array}$ & $\begin{array}{l}\text { INIS-SU-125 p.300 } \\
\text { 32. Conf Nucl Spec- } \\
\text { troscopy. \& Nucl } \\
\text { Structure, } \\
\text { Kiev(1982) } \\
\text { N.S.Biryukov et al }\end{array}$ & $\begin{array}{l}\text { light nuclei, } \\
\text { charge-exchange } \\
\text { reactions, } \\
\text { neutron spectra }\end{array}$ \\
\hline${ }^{58} \mathrm{Ni}$ & $\begin{array}{l}\text { De-Excitation of Continuum } \\
\text { States Induced by Medium } \\
\text { Energy Protons }\end{array}$ & $\begin{array}{l}\text { Int Sym on Highly } \\
\text { Excited States in } \\
\text { Nuclear Reactions, } \\
\text { Osaka,Japan(May 12, } \\
\text { 1980) D.L.Hendrie }\end{array}$ & $\begin{array}{l}\text { inelastic } \\
\text { scattering }\end{array}$ \\
\hline $6,7 \mathrm{Li}$ & $\begin{array}{l}(\mathrm{p}, \mathrm{px}) \text { Reaction on } \\
\text { the } 6 \mathrm{Li} \text { and } 7_{\mathrm{Li}} \text { Nuclei } \\
\text { at the } 70 \mathrm{MeV} \text { Incident } \\
\text { Protons }\end{array}$ & $\begin{array}{l}\text { INIS-SU-125 p.297 } \\
\text { 32. Conf Nucl Spec- } \\
\text { troscopy \& Nucl } \\
\text { Structure, } \\
\text { Kiev (1982) } \\
\text { O.K.Gorpinich et al }\end{array}$ & $\begin{array}{l}\text { knock-out } \\
\text { reactions }\end{array}$ \\
\hline${ }^{9} \mathrm{Be},{ }^{7} \mathrm{Li}$ & $\begin{array}{l}\text { Characteristics of Neutrons } \\
\text { from Be Targets Bombarded } \\
\text { with Protons, Deuterons, } \\
\text { and Alpha Particles }\end{array}$ & $\begin{array}{l}\text { Nucl Instr \& Meth } \\
189,515(1981) \\
\text { M.A.Lone et al }\end{array}$ & $\begin{array}{l}\text { charge-exchange } \\
\text { reactions, } \\
\text { neutron spectra }\end{array}$ \\
\hline${ }^{27} \mathrm{Al}, 63 \mathrm{Cu}$ & $\begin{array}{l}\text { Excitation Functions for } \\
\text { Radioactive Isotopes Pro- } \\
\text { duced by Proton Bombardment } \\
\text { of } \mathrm{Cu} \text { and } \mathrm{Al} \text { in the Energy } \\
\text { Range of } 16 \text { to } 70 \mathrm{MeV}\end{array}$ & $\begin{array}{l}\text { Nucl Phys A383,98 } \\
\text { (1982) A.Gruetter }\end{array}$ & $\begin{array}{l}\text { inclusive } \\
\text { interactions }\end{array}$ \\
\hline $\begin{array}{l}27 \mathrm{Al}, 209 \mathrm{Bi} \\
12 \mathrm{C}, 64 \mathrm{Ci} \\
208 \mathrm{~Pb}\end{array}$ & $\begin{array}{l}\text { How Important Is The First } \\
\text { Inelastic Collision in } \\
\text { Nuclear Precompound } \\
\text { Reactions }\end{array}$ & $\begin{array}{l}\text { Nucl Phys A384,24 } \\
\text { (1982) W.Q.Chao } \\
\text { et al }\end{array}$ & $\begin{array}{l}\text { quasi-elastic } \\
\text { scattering, } \\
\text { inclusive } \\
\text { interactions }\end{array}$ \\
\hline
\end{tabular}


Table I (Cont.)

\section{$6,7 \mathrm{Li}$}

$120 \mathrm{Sn}$

$208 \mathrm{~Pb}$

$\mathrm{Co}, \mathrm{Fe}$

$\mathrm{Cu}, \mathrm{Ni}$

$48,50 \mathrm{Ti}$

$54 \mathrm{Fe}$

$208 \mathrm{~Pb}$
Nucleon Scattering from

Light Nuclei. Pt. 1.

The Target $6 \mathrm{Li}$ and $7 \mathrm{Li}$

Calculation of Neutron,

Proton and Alpha-

Particles Absorption Cross

Sections by Nuclei in the

Framework of Optical Model

and Analytical Formulae

Theory of Statistical

Multi-Step Direct and

Compound Reactions

Semi-Microscopic Optical

Potential Calculation by

Nuclear Matter Approach

Integral Excitation Func-

tions for Proton and

Alpha Induced Reactions

on Target Elements 22

$<=\mathrm{Z}<=28$

48 $\mathrm{Ti}$ and $50 \mathrm{Ti}(p, x p y n)$

Excitation Functions

Induced by Protons from

5 to $85 \mathrm{MeV}$ : Comparison

with Pre-Equilibrium

Calculations

Preequilibrium Multiparticle Calculations

of Nuclear Reactions

Physical Foundation of

Preequilibrium Models

and Open Questions

Relaxation Phenomena in Nucleon-Induced Reactions and Preequilibrium Angular Distributions
Nucl Phys A383,355

(1982) F.Petrovich

et al

5. Nat Sov Conf on

Neutron Physics

Kiev, Sep 15, 1980

INIS-SU-109,p.286

V.M.Bychkov et al

Int Symp on Highly

Excited States in

Nuclear Reactions

Osaka,Japan May

12,1980,p.5,H.Feshbach

Zeit fur Physik

A303,69(1981)

S.Qingbiao et al

Koeln Univ(w.Germany)Thesis, INIS-

MF-7112(1979)

G.Brinkmann

McGill Univ., Montreal,Can.

Thesis (1980)

K.I.Bums

2. Int Sym on Neut. Induced Reactions, Smolenice, Czech. June 25, 1979,p.201 E.Betak, J.Dobes

2. Int Sym on Neut. Induced Reactions, Smolenice, Czech. June 25, 1979,p. 165 E.Gadioli et al

Nucl Phys A373,27 (1982) P.Maedler, R.Reif charge-exchange, elastic and inelastic scattering

optical models, absorption

charge-exchange reactions, direct reactions, statistical models

optical models

excitation

functions, knock-out reactions, quasi-elastic scattering

quasi-elastic scattering, precompoundnucleus emission

precompoundnucleus emission, photon emission

precompoundnucleus emission, data compilation, spallation

thermodynamic properties, time dependence, transport theory 


$\begin{array}{ll}11_{\mathrm{B}},{ }^{12} \mathrm{C} & \begin{array}{l}\text { Single-Particle Model of } \\ \text { Capture to Continuum } \\ \text { States }\end{array} \\ 238 \mathrm{U} & \begin{array}{l}238 \mathrm{U} \text { Fission at Energics } \\ \text { Up to } 100 \mathrm{MeV}\end{array} \\ 208 \mathrm{~Pb} & \begin{array}{l}\text { To The Problem on the } \\ \text { Ratio of Contributions } \\ \text { of Multistep Direct and } \\ \text { Pre-Equilibrium Processes } \\ \text { in Inelastic Nucleon } \\ \text { Scattering }\end{array}\end{array}$

$\mathrm{Fe}, \mathrm{Ni}, \mathrm{Ti}$

$50 \mathrm{Ti}$

$\mathrm{Ge}, \mathrm{Ga}$

$27 \mathrm{Al}$

$208 \mathrm{Bi}, 12 \mathrm{C}$

$208 \mathrm{~Pb}$

$12 \mathrm{C}, 63 \mathrm{Cu}$

$6,7_{\mathrm{Li}}$

$235,236 \mathrm{U}$

$238 \mathrm{U}$

${ }^{9} \mathrm{Be}$
Isospin Dependence of the $65 \mathrm{MeV}$ Proton Optical Potential in $\mathrm{f}-\mathrm{p}$ Shell Nuclei

Emission of Alpha Particles in the interaction of 10-85 $\mathrm{MeV}$ Protons with $48,50 \mathrm{Ti}$

Proton Scattering by Nuclei of Atoms of Semiconductors and Energy Spectra of Primarily Knocked Out Atoms

Spectral Measurements of Neutrons Produced by $52 \mathrm{MeV}$ Protons with Activation Detectors

Backward Inclusive Spectra in Proton-Nucleus Collisions at $640 \mathrm{MeV}$

Postfission Neutrons from Proton Induced Fission

Neutron Yields from Proton,Deuteron and Alpha Bombardment of Beryllium of $235,236,238 \mathrm{U}$
Nucl Phys A375, 169 (1982) R.J.Philpott, D.Halderson

Phys Rev C25,899 (1982) C.Chung, J.J.Hogan

INIS-MF-6819 p.490 31. Conf Nucl Spectroscopy \& Nucl Structure,Samarkland, USSR April 14, 1981,F.A.Zhivopistsev, N.P.Yudin

Nucl Phys A366, 189 (1981) T.Moro et al

Zeit fur Physik A301, 289(1981) E.Gadioli et al

INIS-MF-6819 p.557 31. Conf Nucl Spectroscopy \& Nucl Structure,Samarkand, USSR April 14, 1981 I.D.Fedorets et al

Nucl Instr \& Meth 187, 587(1981) K.Shin et al

Nucl Phys A367,419 (1981) J.Eroe et al

Nuclear Energy Agency report NEANDC(E)-212U V5,p.37 (1980)P.Plischke et al

Consultants Mtg on Neutron Source Properties,Debrechen, Hungary,INDC (NDS)-114/GT,p308 (1980) M.A.Lone, B.C.Robertson capture, inelastic scattering

spallation, fission

inelastic scattering, direct reactions, precompoundnucleus emission

elastic scattering

knock-out reactions

recoils, energy spectra

spectra unfolding

inclusive interactions

neutron spectra

charge-exchange reactions 
Table I (Cont.)

$\begin{array}{ll}{ }^{232} \mathrm{Th} & \begin{array}{l}\text { K-Shell Ionisation of } \\ \text { 232Th by Protons and } \\ \text { Alpha Particles }\end{array} \\ \mathrm{Co,Ni} & \begin{array}{l}\text { Pre-Equilibrium Emission } \\ \text { in Proton-Induced Reactions } \\ \text { on Cobalt and Nickel in } \\ \text { the Energy Range 18.84 MeV }\end{array}\end{array}$

$27 \mathrm{Al}, 12 \mathrm{C}$ $56 \mathrm{Fe}$ $181 \mathrm{Ta}$

$12 \mathrm{C}, 14 \mathrm{~N}$

$16 \mathrm{O}$

$92 \mathrm{Mo}$

$27 \mathrm{Al}$

$232 \mathrm{Th}$

$27 \mathrm{Al}, 59 \mathrm{Co}$ $58 \mathrm{Ni}$

$120 \mathrm{Sn}$
One, Two, Infinity: A

Pragmatic Approach to

Nuclear Precompound

Reactions

Properties of Quasielastic

Knockout of Slow Protons

( $E<$ or Approx. $=10 \mathrm{MeV}$ )

From $C, N$, and $O$ Nuclei

by High-Energy Protons

Energy Dependence in Proton Inelastic Scattering

What Happens When $A$ Fast Nucleon Goes Through A Nucleus

Fission Probability of the Reaction 232Th(p,p'f) at $E_{p}=52.5 \mathrm{MeV}^{\prime}$

Symmetric and Asymmetric Components of Energy Spectra in a Preequilibrium Decay

Statistical Multistep Direct Emission in the $120 \mathrm{Sn}(\mathrm{p}, \mathrm{n})$ Reaction
J of Physics B14, 2391(1981)

P.Hornshoj et al

FRNC-CONF-201, Int Conf on Nucl Behavior at High Angular Momentum,Strassbourg,Fr. April 22, 1980 S.J.Mills, F.J.Haasbroek

Nucl Phys A349,466 (1980) H.C.Chiang, J.Huefner

Sov J Nucl Physics 31,295(1980)

A.A.Levkovskii, E.F.Dreiman

Nucl Phys A352,221 (1981) R.A.Moyer et al

Phys Lett B100,101 (1981) E.F.Redish, D.M.Schneider

Acta Physica Austriaca 52, 125(1980) G.Elder et al

INIS-MF-6160 p.500 30. Conf on Nuclear Spectroscopy \& Nucl. Structure Leningrad, March 18, 1980, F.A.Zhivopistsev et al

Phys Lett B94,463 (1980) L.Avaldi et al k-shell, ionization

precompoundnucleus emission

inclusive interactions, precompoundnucleus emission

knock-out reactions. quasi-elastic scattering

elastic and inelastic scaltering, optical models

mean free path

inelastic scattering

precompoundnucleus emission

charge-exchange reactions 


\begin{tabular}{|c|c|c|c|}
\hline $\begin{array}{l}12 \mathrm{C}, 197 \mathrm{Au} \\
58 \mathrm{Ni}\end{array}$ & $\begin{array}{l}\text { Pickup in the Surface - } \\
\text { The Origin of Deuterons } \\
\text { in Nuclear Precompound } \\
\text { Reactions }\end{array}$ & $\begin{array}{l}\text { Phys Lett B97,183 } \\
(1980) \text { F.Hachenberg } \\
\text { et al }\end{array}$ & $\begin{array}{l}\text { precompound- } \\
\text { nucleus emission, } \\
\text { pickup reactions }\end{array}$ \\
\hline Al & $\begin{array}{l}\text { Excitation Functions of } \\
(p, t . . .),(d, t . .) \\
\text { (tau,t...) and (alpha,t...) } \\
\text {-Reactions and Comparison } \\
\text { with Results of Exciton } \\
\text { Model Calculations }\end{array}$ & $\begin{array}{l}\text { Verhandlungen Der } \\
\text { Deutschen Physik- } \\
\text { alischen Gesell- } \\
\text { schaft (E.Ger.) } \\
\text { 16,790(1981) } \\
\text { M.Merkel,H.Muenzel }\end{array}$ & $\begin{array}{l}\text { pickup reactions, } \\
\text { exciton model }\end{array}$ \\
\hline${ }^{63} \mathrm{Cu}, 62 \mathrm{Ni}$ & $\begin{array}{l}\text { Preequilibrium Emission } \\
\text { of Nucleons and Alpha } \\
\text { Particles from } 63 \mathrm{Cu} \\
\text { and } 64 \mathrm{Zn}\end{array}$ & $\begin{array}{l}\text { Verhandlungen Der } \\
\text { Deutschen Physik- } \\
\text { alischen Gesell- } \\
\text { schaft(E.Ger.) } \\
16,791(1981) \\
\text { A.Alevra et al }\end{array}$ & $\begin{array}{l}\text { precompound- } \\
\text { nucleus emission }\end{array}$ \\
\hline \multirow[t]{2}{*}{$\begin{array}{l}54 \mathrm{Fe} \\
208 \mathrm{~Pb}\end{array}$} & $\begin{array}{l}\text { Semimicroscopic Analysis } \\
\text { of Direct Process Contri- } \\
\text { bution to Proton Inelastic } \\
\text { Scattering Spectra }\end{array}$ & $\begin{array}{l}\text { Yad Fiz } 30,1258 \\
\text { (1979) A.I.Blokhin, } \\
\text { V.G.Pronyaev }\end{array}$ & $\begin{array}{l}\text { inelastic } \\
\text { scattering }\end{array}$ \\
\hline & $\begin{array}{l}\text { Calculation of Proton- } \\
\text { Induced Radioisotope Pro- } \\
\text { duction Yields with a } \\
\text { Statistical-Model Based } \\
\text { Code }\end{array}$ & $\begin{array}{l}\text { Int J of Applied } \\
\text { Radiation \& Iso- } \\
\text { topes } 32,73(1981) \\
\text { R.Nowotny }\end{array}$ & statistical models \\
\hline $232 \mathrm{Th}$ & $\begin{array}{l}\text { Fission of } 232 \mathrm{Th} \text { at } \\
\text { Energies Up to } 90 \mathrm{MeV}\end{array}$ & $\begin{array}{l}\text { Phys Rev C24, } 180 \\
\text { (1981) C.Chung, } \\
\text { J.J.Hogan }\end{array}$ & $\begin{array}{l}\text { evaporation model, } \\
\text { spallation }\end{array}$ \\
\hline $93 \mathrm{Nb}$ & $\begin{array}{l}\text { Multistep Direct Reaction } \\
\text { Analysis of Analyzing } \\
\text { Powers of Continuum Spectra } \\
\text { in (p,alpha) Reactions }\end{array}$ & $\begin{array}{l}\text { Phys Rev C23, } 2769 \\
\text { (1981)T.Tamura } \\
\text { et al }\end{array}$ & $\begin{array}{l}\text { three-nucleon } \\
\text { transfer }\end{array}$ \\
\hline $\begin{array}{l}48 \mathrm{Ca} \\
208 \mathrm{~Pb}\end{array}$ & $\begin{array}{l}\text { Analysis of Precompound } \\
\text { Processes in }(p, n) \\
\text { Reactions with the Statis- } \\
\text { tical Multistep Direct } \\
\text { Emission Theory }\end{array}$ & $\begin{array}{l}\text { Phys Rer C24,71 } \\
\text { (1981) R.Bonetti } \\
\text { et al }\end{array}$ & $\begin{array}{l}\text { charge-exchange } \\
\text { reactions, } \\
\text { precompound- } \\
\text { nucleus emission }\end{array}$ \\
\hline $\begin{array}{l}59 \mathrm{Co} \\
{ }^{197} \mathrm{Au}\end{array}$ & $\begin{array}{l}\text { Proton-Induced Pre-Equili- } \\
\text { brium Alpha/Tau-Emission } \\
\text { for } E_{(p)}=72 \mathrm{MeV}\end{array}$ & $\begin{array}{l}\text { Helv Phys Acta } 52 \text {, } \\
\text { 421,(1980) Z.Lewan- } \\
\text { dowski et al }\end{array}$ & pickup reaction. \\
\hline
\end{tabular}


$\mathrm{Be}$

$\mathrm{Ni}$

$58 \mathrm{Ni}$

$27 \mathrm{Al}$

$209 \mathrm{Bi}$

$58 \mathrm{Ni}$

${ }^{119} \mathrm{Sn}$

$208 \mathrm{~Pb}$

$209 \mathrm{Bi}$

$232 \mathrm{Th}$

$238 \mathrm{U}$

$106 \mathrm{Cd}$

$61 \mathrm{Ni}$
Improved Measurement of

Neutron Spectrum from

$35 \mathrm{MeV}$ Protons on Thick

Beryllium

Damage Analysis and

Fundamental Studies

Quarterly Report

Energy Dissipation Process for $100-\mathrm{MeV}$ Protons and the Nucleon-Nucleon Interactions in Nuclei

Proton and Neutron Inclusive Spectra and the Importance of the Single Nucleon-Nucleon Scattering Process

Angular Distributions of Pre-equilibrium Reactions Within a Generalized Exciton Model

Fissionability and Charge Dispersion Studies of Thorium by Protons of Energy to $90 \mathrm{MeV}$

Estimation of Neutron Yield from Individual Fragments in MediumExcitation Fission

Measurement of CompoundNucleus Lifetime by X-Ray Spectroscopy in the 106Cd Reaction

Preequilibrium Angular

Distributions in the Quasi-Free Scattering

Model

Systematics of Particle-

Nucleus Reactions

1. Parameters
Medical Physics $\underline{8}$, 396(1981)

J.L.Ullmann et al

Battelle Pacific

Northwest Lab report BNL-SA-9039 (1980) R.H Jones

Phys Rev Lett 4 S, 1930 (1980)

A.A.Cowley et al

Phys Rev Lett 46,

226 (1981)

B.D.Anderson et al

Nucl Phys A337,445

(1980) P.Maedler,

R.Reif

McGill Univ.

Montreal,Quebec

Thesis(1980)

C.Chung

J Nucl Sci \& Tech

16,466(1979)

H.Yamamoto et al

Nucl Phys 1331,407

(1979) J.F.Chemin

et al

INIS-MF-5692 p.

40, Annual Report

1977-1978

J.Bisplinghoff et al

Phys Rev C22,2116

(1980) L. Winsberg charge-exchange

reactions, neutron spectra

physical radiation effects

quasi-elastic scattering, impulse approximation

inclusive interactions

precompoundnucleus emission

precompoundnucleus emission, exciton model

fission fragments, neutron emission

inelastic scattering $x$ radiation

precompoundnucleus emission, inclusive interactions

fission, fragmentation, spallation, energy spectra 
Table I (Cont.)

${ }^{12} \mathrm{C}$

$12 \mathrm{C}$

$197 \mathrm{Au}$

$279 \mathrm{~Pb}, \mathrm{Lu}$

$181 \mathrm{Ta}$

165ito

$107 \mathrm{Ag}$

$169 \mathrm{Tm}$

$54,56 \mathrm{Fe}$

$12 \mathrm{C}, 14 \mathrm{~N}$

160

$181 \mathrm{Ta}$

${ }^{197} \mathrm{Au}$

$181 \mathrm{Ta}$

$209 \mathrm{Bi}$

$54,56 \mathrm{Fe}$

$27 \mathrm{Al}$
${ }^{12} \mathrm{C}(\mathrm{p}, \mathrm{n}){ }^{12} \mathrm{~N}$ Reaction at $99 \mathrm{MeV}$

Atomic-Excitation Effects on Nuclear Reactions

Study of K X-Ray Nuclear

Satellites from $(p, x n)$

Reactions on Heavy Elements

Alpha Emission in Proton

Induced Reactions

Solution of Nuclear Kinetic

Equations for Quasiequili-

brium System andiDescrip-

tion of Energy Spectra

of Secondary Nucleons

Cross Sections of the

$50 \mathrm{MeV}$ Proton Reactions

on the $12 \mathrm{C}, 14 \mathrm{~N}, 16 \mathrm{O}$

$\mathrm{Ni}$ :lei

Statistical Theory of

Multi-Step Compound and

Direct Reactions

Measurement of the Proton

Total Reaction Cross Sec-

tion for ${ }^{159} \mathrm{~Tb}, 181 \mathrm{Ta}$, and

${ }^{197} \mathrm{Au}$ Between 20 and $48 \mathrm{MeV}$

Quasiclassical Method of

Nuclear Kinetic Equation

Solution and Description

of Nucleon Angular

Distributions

Proton-Induced Pre-Equili-

brium Alpha-Emission at

$\mathrm{Ep}=72 \mathrm{MeV}$
Phys Rev C22, 1826

(1980) J.N.Knudson

et al

Phys Rev Lett 45 ,

703 (1980)

E.J.Ludwig,

E.Merzbacher

J of Physics G6,

117 (1980)

V.Zoran et al

Zeit fur Physik

A293, (1979)

A.Ferrero et al

Bull Acad Sci

Phys Series

42,1893 (1978)

V.E.Bunakov et a!

Bull Acad Sci

Phys Series

43,148 (1979)

A.I.Vdovin et al

Annals of Physics

125,429(1980)

H.Feshbach et al

Nucl Phys A324.

109 (1979) R.Abegg

et al

Bull Acad Sci

Phys Series

42,2351 (1978)

V.E.Bunakov

Helv Phys Acta $\underline{52}$,

88(1979)

Z.Lewandowski et al charge-exchange

reactions,

netitron spectra

recoils

gamma cascades, k-shell, x-ray spectra

particle-hole model, knock-out reactions

evaporation model, neutron spectra, time dependence

direct reactions

statistical

models,

preequilibrium

theory

optical models

precompoundnucleus emission

precompoundnucleus emission 


\begin{tabular}{|c|c|c|c|}
\hline $\mathrm{C}, \mathrm{Cu}, \mathrm{Fe}$ & $\begin{array}{l}\text { Production of Neutrons and } \\
\text { Photons from Thick Targets } \\
\text { Bombarded by } 52 \mathrm{MeV} \text { Protons }\end{array}$ & $\begin{array}{l}\text { 2. Symp Accelerator } \\
\text { Sci \& Tech., Tokyo, } \\
\text { Japan,p.197 (1978) } \\
\text { K.Shin et al }\end{array}$ & $\begin{array}{l}\text { secondary } \\
\text { emission }\end{array}$ \\
\hline $27 \mathrm{Al}$ & $\begin{array}{l}\text { Momentum-Dependent State } \\
\text { Densities in the Exciton } \\
\text { Model }\end{array}$ & $\begin{array}{l}\text { Dresden Univ (E. } \\
\text { Germany) report } \\
\text { ZFK-376,p.,11(1978) } \\
\text { P.Maedler, R.Reif }\end{array}$ & $\begin{array}{l}\text { precompound- } \\
\text { nucleus emission }\end{array}$ \\
\hline $\begin{array}{l}27 \mathrm{Al}, 9 \mathrm{Be} \\
59 \mathrm{Co}, 55 \mathrm{Mn}\end{array}$ & $\begin{array}{l}\text { Proton Elastic Scattering } \\
\text { on Light Nuclei Il. } \\
\text { Nuclear Structure Effects }\end{array}$ & $\begin{array}{l}\text { Phys Rev C21,844 } \\
\text { (1980) E.Fabrici } \\
\text { et al }\end{array}$ & optical models \\
\hline $24 \mathrm{Mg}$ & $\begin{array}{l}\text { Proton Elastic Scattering } \\
\text { on Light Nuclei I. Energy } \\
\text { Dependence }\end{array}$ & $\begin{array}{l}\text { Phys Rev C21,830 } \\
\text { (1980) E.Fabrici } \\
\text { et al }\end{array}$ & optical models \\
\hline $24 \mathrm{C}$ & $\begin{array}{l}\text { Calculation of Z Greater } \\
\text { Than or Equal to } 3 \text { Mass, } \\
\text { Energy and Angular } \\
\text { Distributions in the } \\
p+12 \text { C Reaction }\end{array}$ & $\begin{array}{l}\text { Univ. Maryland } \\
\text { report ORO-5172- } \\
14, \text { p.46(1979) } \\
\text { A.Goekmen et al }\end{array}$ & $\begin{array}{l}\text { evaporation } \\
\text { model, } \\
\text { nuclear cascades }\end{array}$ \\
\hline \multirow[t]{2}{*}{${ }^{59} \mathrm{Co}, \mathrm{Fe}$} & $\begin{array}{l}\text { Measurement and Hybrid-Model } \\
\text { Analysis of Proton-Induced } \\
\text { Reactions with V, Fe and Co }\end{array}$ & $\begin{array}{l}\text { Nucl Phys A322,40 } \\
\text { (1979) R.Michel } \\
\text { et al }\end{array}$ & $\begin{array}{l}\text { precompound- } \\
\text { nucleus emission }\end{array}$ \\
\hline & $\begin{array}{l}\text { Penetration of Secondary } \\
\text { Neutrons and Photons from } \\
\text { a Graphite Assembly Exposed } \\
\text { to 52-MeV Protons }\end{array}$ & $\begin{array}{l}\text { Nucl Sci \& Eng 71, } \\
294 \text { (1979) K.Shin } \\
\text { et al }\end{array}$ & $\begin{array}{l}\text { fast neutrons, } \\
\text { gamma radiation }\end{array}$ \\
\hline $40 \mathrm{Ca}$ & $\begin{array}{l}\text { Nucleon Optical Potential } \\
\text { from a Realistic Inter- } \\
\text { nucleon Potential }\end{array}$ & $\begin{array}{l}\text { J Phys Soc Japan } \\
\text { 44,539(1978) } \\
\text { F.Brieva, I R.Pook }\end{array}$ & $\begin{array}{l}\text { nucleon-nucleon } \\
\text { potential }\end{array}$ \\
\hline${ }^{209} \mathrm{Bi}$ & $\begin{array}{l}(p, n) \text { Reaction on }{ }^{209} \mathrm{Bi} \\
\text { and Pre rompound Processes }\end{array}$ & $\begin{array}{l}\text { J Phys Soc Japan } \\
45,1071(1978) \\
\text { K.Miyano et al }\end{array}$ & $\begin{array}{l}\text { precompound- } \\
\text { nucleus emission }\end{array}$ \\
\hline $165 \mathrm{Ho}$ & $\begin{array}{l}\text { Preequilibrium and Equilib- } \\
\text { rium Deexcitation Processes } \\
\text { for } D y(\text { alpha,xn gamma)Er } \\
\text { Reactions at } E_{\text {alpha }}=90 \\
\mathrm{MeV} \text { and } 165_{\mathrm{Ho}}(\mathrm{p}, \mathrm{xnyp} \text { gamma) } \\
\text { Reactions at } \mathrm{E}_{\mathrm{p}}=60 \mathrm{MeV}\end{array}$ & $\begin{array}{l}\text { J Phys Soc Japan } \\
44,655(1978) \\
\text { H.Ejiri et al }\end{array}$ & $\begin{array}{l}\text { neutron spectra } \\
\text { precompound- } \\
\text { nucleus emission }\end{array}$ \\
\hline
\end{tabular}


$28,30 \mathrm{Si}$

${ }_{197} \mathrm{Au}$

$9_{\mathrm{Be}}$

Sn, Ti

W, Y

$27 \mathrm{Al}$

$165 \mathrm{Ho}$

Gd, Th

$\mathrm{Cu}, \mathrm{Eu}$

$\mathrm{Ag}$

$79,81 \mathrm{Br}$

${ }^{56} \mathrm{Fe}$
Asymmetry in the Elastic and Inelastic Scattering of $65 \mathrm{MeV}$ Protons from $28 \mathrm{Si}$, ${ }^{29} \mathrm{Si}, 30 \mathrm{Si}, 31 \mathrm{P},{ }^{89} \mathrm{Y}$ and $90 \mathrm{Zr}$

Fissionability of Nuclides in the Thorium Region at Excitation Energies

to $100 \mathrm{MeV}$

Preequilibrium AlphaEmission in the $197 \mathrm{Au}$ (p,alpha $x$ )-Reaction at $\mathrm{Ep}=72 \mathrm{MeV}$

Neutron Spectra from 35 and $46 \mathrm{MeV}$ Protons, 16 and 28 $\mathrm{MeV}$ Deuterons, and $44 \mathrm{MeV}$ 3He Ions on Thick Beryllium

Contribution to the Study of Ionization of Elements by Protons and Alpha Particles of 4 to $30 \mathrm{MeV}$

Measurement and Analysis of Continuous Alpha Spectra in Proton Induced Reactions

Relativistic Effects in KShell Ionisation by 7-15 MeV Proton Bombardment on Heavy Elements

Atomic K-Shell Ionization Induced by $20-50 \mathrm{MeV}$ Protons

${ }^{79} \mathrm{Br}$ and ${ }^{81} \mathrm{Br}(\mathrm{p}, \mathrm{xn})$ and (p,pxn) Excitation Functions in the Energy Range 10-85 MeV

Gamma-Ray Production Cross Sections
J Phys Soc Japan

44,557(:978)

M.Nakamura et al

Pliys Rev C.20,1831

(1979) J.J.Hogan

et al

Phys Lett B80, 350 (1979)

Z.Lewandowski et al

Medical Physics 6 ,

432 (1979)

F.M.Waterman et al

Nucl Instr \& Meth

159,455(1979)

M.Poncet,

Ch.Engelmann

Canf on Clustering

Asp cts of Nuclear

st. cture \& Nuclear

iveactions, Winnipeg

Canada (1978)

A.Ferrero et al

J of Phys B11,2875

(1978) A.Berinde

et al

Phys Lett A69,258

(1978) W.D.Ramsay

et al

Nucl Phys A304,40

(1979) M.Diksic

et al

Washington Univ

Annual report

DOE/ER-01388-345

n.51(1976)

D.Bodansky et al elastic, inelastic scattering

spallation, neutron spectra

precompoundnucleus emission

neutron spectra

ionization, K, L, M Shell

precompoundnucleus emission

inner-shell ionization

inner-shell icnization

precompoundnucleus emission

charge-exchange reactions, inelastic scattering 
${ }^{11_{\mathrm{B}}}{ }^{12} \mathrm{C}$

165 Ho

$208 \mathrm{~Pb}$

$27 \mathrm{Al}$

$58 \mathrm{Ni}$

$90 \mathrm{Zr}$

$12 \mathrm{C}$

$14 \mathrm{~N}$

$208 \mathrm{~Pb}$

$\mathrm{Ni}$

$12 \mathrm{C}, 14 \mathrm{~N}$

160

$54 \mathrm{Fe}$

$65 \mathrm{Cu}$

$\mathrm{C}, \mathrm{Cu}$,

$\mathrm{Pb}, \mathrm{Fe}$
Radiative-Emission Spec-

trum of 50-MeV Nuclear

Excitations Populated

by Proton Capture

Gamma Rays and Neutrons

Following Preequilibrium

165Ho(p,xnp gamma) Reac-

tions at $\mathrm{Ep}=60 \mathrm{MeV}$

Simple Unitary Estimate

for Multistep Processes

Charged-Particle Spectra:

$90 \mathrm{MeV}$ Protons on $27 \mathrm{Al}$,

$58 \mathrm{Ni}, 90 \mathrm{Zr}$, and $209 \mathrm{Bi}$

Cross Section of $50 \mathrm{MeV}$

Proton Reactions on $12 \mathrm{C}$, $14 \mathrm{~N}, 160$ Nuclei with

Escape of Four Charged

Particles

Integral Contribution of

Direct Processes Irielastic

Scattering Spectra and the

Preequilibrium Decay Model

Proton-Induced Reactions

on Nickel with Energies

Between 12 and $45 \mathrm{MeV}$

Cross Sections of $50 \mathrm{MeV}$

Proton Induced Reactions

on $12 \mathrm{C}, 14 \mathrm{~N}$, and $16 \mathrm{O}$

Nuclei with Three Charged

Particles in the Final State

Study of Two-Particle

Nuclear Reactions in

Framework of the

Exciton Model

Spectral Measurements of

Neutrons and Photons from

Thick Targets of $\mathrm{C}, \mathrm{Fe}, \mathrm{Cu}$

and $\mathrm{Pb}$ by $52 \mathrm{MeV}$ Protons
Phys Rev Lett 42 ,

1253 (1979)

L.G.Arnold

Nucl Phys A305,

167 (1978) H.Ehiri

et al

Phys Rev C19,693

(1979) S.R.Cotanch

Phys Rev $\underline{\mathrm{C} 19}, 698$

(1979) J.R.Wu

et al

INIS.MF-4388 p.I39

(1978) 28. Conf Nucl

Spec \& Structure Atomic

Nucleus Alma-Ata,USSR

A.I.Vdovin et al

INIS-MF-4468 pp.

130-134 (1977) 4th

All-Union Conf

Neutron Physics

A.l.Blu'hin et al

Zeit fur Physik

A286,393(1978)

R.Michel et al

Sov J Nucl Phys

27,3 (1978)

I.G.Golikov et al

Ukr Phys J 22,

202 I(1977) S.N.

Ezhov, V.A.Plyuyko

Nucl Instr \& Meth

151,493(1978)

T.Nakamura et al gamma spectra

precompound-

nucleus emission, gamma spectra

pir ap reactions

precompoundnucleus enission

precompound nucleus emission

precompoundnucleus emission

breakup reactions

compound-nucleus reactions, inelastic

scattering

neutron spectra 
$\begin{array}{ll}{ }^{54} \mathrm{Fe} & \text { Cascade-Exciton Model for } \\ \text { Nuclear Reactions }\end{array}$

$181 \mathrm{Ta}$

$\mathrm{Li}$

$40 \mathrm{Ca}$

$56 \mathrm{Fe}$

${ }^{209} \mathrm{Bi}$

$\mathrm{Ni}, \mathrm{Nb}$

${ }^{7 \mathrm{Li}}$

$27 \mathrm{Al}$

$209 \mathrm{Bi}$

$197 \mathrm{Au}$

${ }^{54} \mathrm{Fe}$
Neutron Spectra and Angular Distributions from the Reactions $181 \mathrm{Ta}(\mathrm{p}, \mathrm{n})$ $181 \mathrm{~W}$,and $181 \mathrm{Ta}\left(\mathrm{n}, \mathrm{n}^{\prime}\right) 181 \mathrm{Ta}$

Neutron Spectra from Deuteron and Proton Bomiardment of Thick Lithium Targets

Two-Step Processes in Inelastic Proton Scattering

Use of the Intranuclear Cascade Model for Inciderit Nucleons of Intermediate and Low Energy

Calculation of Energy Spectra of Primary Knock-On Atoms in the Proton Elastic Scattering by the Nuclei of Different Elements

Neutron Spectra from Deuteron and Proton Bombardment of Thick Lithium Targets: Potential for Neutron Therapy

Complex-Particle Enission in the Pre-Equilibrium Exciton Model

Multi-jtep-Direct-Reaction Analysis of Continuum Spectra of (p,alpha) Reaction

Pre-Equilibrium Emission
Dresden Univ (E.

Germany) report

ZFK-324,p.79(1976)

K.K.Gudima,

V.D.Toneev

Sov J Nucl Phys

26,606(1977)

N.S.Biryukov

et al

Brookhaven report

BNL-NCS-125 (1977)

C.E. Nelson et al

Zeit fur Physik

A284,413(1978)

E.F.Hefter

Phys Lett 73,267

(1978) V.E.Bunakov

et al

Kharkov Physics-

Tech. Instit.

(Ukraine) report

FTI-76-45,p.29 (1976)

B.A.Shilyaev et al

Phys in Medicine \&

Biology 23,39(1978)

C.ENelson et al

Phys Rev C17, 1540

(1978) J.R.Wu,

C.C.Chang

Phys Lett B71,273

(1977) T.Tamura,

T.Udagawa

Nucl Instr \& Meth

146,265(1977)E.

Gadioli, E.E.Gadioli direct reactions, precompoundnucleus emission, nuclear cascades

neutron spectra, precompoundnucleus emission

energy spectra

multiple

scattering

nuclear cascades

optical models

fast neutrons

precompoundnucleus emission

pickup reactions

precompoundnucleus emission, excitons 


\begin{tabular}{|c|c|c|c|}
\hline $\begin{array}{l}140 \mathrm{Ce} \\
24 \mathrm{Mg} \\
64 \mathrm{Zn} \\
66,68 \mathrm{Zn}\end{array}$ & $\begin{array}{l}\text { Measurements of the Proton } \\
\text { Total Reaction Cross } \\
\text { Section for } 24 \mathrm{Mg} \text {, } 64,66 \text {, } \\
68 \mathrm{Zn} \text { and } 140 \mathrm{Ce} \text { Between } \\
17.5 \text { and } 48 \mathrm{MeV}\end{array}$ & $\begin{array}{l}\text { Nucl Phys } \hat{A} 290,45 \\
\text { (1977) N.E.Davison } \\
\text { et al }\end{array}$ & $\begin{array}{l}\text { ?'tal cross } \\
\text { sections }\end{array}$ \\
\hline $\begin{array}{l}88 \mathrm{Sr} \\
89 \mathrm{Y}, 90 \mathrm{Zr}\end{array}$ & $\begin{array}{l}\text { Pree puilibrium Decay of } \\
\text { Nuclei with A Approx. = } \\
90 \text { at Excitation } \\
\text { Energies to } 100 \mathrm{MeV}\end{array}$ & $\begin{array}{l}\text { Phys Rev C16,1404 } \\
\text { (1977) E.Gadioli } \\
\text { et al }\end{array}$ & $\begin{array}{l}\text { precompound- } \\
\text { nucleus emission }\end{array}$ \\
\hline $\begin{array}{l}209 \mathrm{Bi} \\
54 \mathrm{Fe}\end{array}$ & $\begin{array}{l}\text { A-Priori Pre-Equilbrium } \\
\text { Decay Models }\end{array}$ & $\begin{array}{l}\text { Nukleonika } 19,183 \\
\text { (1974) M.Blann }\end{array}$ & $\begin{array}{l}\text { precompound- } \\
\text { nucleus emission, } \\
\text { nucleon-nucleon } \\
\text { interactions }\end{array}$ \\
\hline $\begin{array}{l}27 \mathrm{Al} \\
56 \mathrm{Fe} \\
\mathrm{Si}, \mathrm{S}\end{array}$ & $\begin{array}{l}\text { Empirical Observations } \\
\text { About the Nucleon Optical } \\
\text { Model Potential }\end{array}$ & $\begin{array}{l}\text { Phys Lett B70,161 } \\
\text { (1977) J Rapaport }\end{array}$ & $\begin{array}{l}\text { elastic } \\
\text { scattering, } \\
\text { optical models }\end{array}$ \\
\hline $14 N, 160$ & $\begin{array}{l}\text { Optical Model and Proton } \\
\text { Elastic Scattering from } \\
14 \mathrm{~N} \text { and } 160\end{array}$ & $\begin{array}{l}\text { Aust J Physics } 30 \text {, } \\
\text { 287(1977) A.Gabric, } \\
\text { K.Amos }\end{array}$ & $\begin{array}{l}\text { elastic } \\
\text { scattering, } \\
\text { optical models }\end{array}$ \\
\hline $\mathrm{Cs}$ & $\begin{array}{l}\text { Proton Loss by Nuclear } \\
\text { Inelastic Interactions } \\
\text { in CsI, Ne102, Ne213 } \\
\text { and Stilbene }\end{array}$ & $\begin{array}{l}\text { Nucl Instr \& Meth } \\
143,58^{\circ}(1977) \\
\text { AM.Sourkes et al }\end{array}$ & $\begin{array}{l}\text { plastic } \\
\text { scintillators } \\
\text { inelastic } \\
\text { scattering }\end{array}$ \\
\hline $89 Y$ & $\begin{array}{l}\text { Influence of Nucleon Mean- } \\
\text { Free Paths on Intranuclear } \\
\text { Cascade Results }\end{array}$ & $\begin{array}{l}\text { Phys Lett B68,405 } \\
\text { (1977) J.Ginocchio, } \\
\text { M.Blann }\end{array}$ & $\begin{array}{l}\text { inelastic } \\
\text { scattering, } \\
\text { nuclear cascades, } \\
\text { nuclear matter }\end{array}$ \\
\hline $\begin{array}{l}15 \mathrm{~N} \\
40 \mathrm{Ca}\end{array}$ & $\begin{array}{l}\text { Influence of Recoil } \\
\text { Effects in the Micro- } \\
\text { scopic Description in } \\
\text { Inelastic Scattering }\end{array}$ & $\begin{array}{l}\text { Phys Lett B69,261 } \\
\text { (1977) HP.Blok, } \\
\text { P.D.Kunz }\end{array}$ & $\begin{array}{l}\text { inelastic } \\
\text { scattering, } \\
\text { recoils }\end{array}$ \\
\hline $\begin{array}{l}6.7 \mathrm{Li} \\
9 \mathrm{Be}\end{array}$ & $\begin{array}{l}\text { Study of the (p,pn) } \\
\text { Reaction in Light } \\
\text { Nuclei }\end{array}$ & $\begin{array}{l}\text { Nucl Phys A281, } 418 \\
\text { (1977) C.N.Waddell } \\
\text { et al }\end{array}$ & $\begin{array}{l}\text { impulse } \\
\text { approximations, } \\
\text { knock-out } \\
\text { reactions, breakup } \\
\text { reactions }\end{array}$ \\
\hline${ }^{197} \mathrm{Au}$ & $\begin{array}{l}\text { Compact Modified Exciton } \\
\text { Model for the Pre-Equili- } \\
\text { brium Decay of Highly } \\
\text { Excited Nuclei }\end{array}$ & $\begin{array}{l}\text { Kernenergie 20, } 180 \\
\text { (1977) P.Maedler }\end{array}$ & $\begin{array}{l}\text { precompound- } \\
\text { nucleus emission }\end{array}$ \\
\hline
\end{tabular}




\section{${ }^{9} \mathrm{Be}^{7}{ }_{\mathrm{Li}}$}

$64,66 \mathrm{Zn}$

$68,70 \mathrm{Zn}$

$27 \mathrm{Al}$

$209 \mathrm{Bi}$
Thick Target Neutron Yields and Spectral Distributions from the $7 \mathrm{Li}(d(p), n)$ and

${ }^{9} \mathrm{Be}(\mathrm{d}(\mathrm{p}), \mathrm{n})$ Reactions

Compound and Precompound Processes in (p,p') and (alpha,p) Reactions of Even Zn Isotopes

Deep Inelastic Reactions

Treated as Multi-Step

Direct Reaction Processes.

Application to (p,p')

Reaction

Study of the Pure Continuum in Inelastic Proton

Scattering

\section{Energy Spectra of Recoil} Atoms While Irradiating Different Elements by Fast Protons and Neutrons
$12 \mathrm{C}, 14 \mathrm{~N}$

\author{
Deuteron Break-Up Effects \\ in $(p, d)$ Reactions \\ at $65 \mathrm{MeV}$
}

Description of Secondary Nuclear Energy Spectra Produced in Low and Intermediate Energy Proton Interaction with Nuclei

\section{${ }^{7 \mathrm{Li}, 9 \mathrm{Be}}$}

Nucl Instr \& Meth 143,331(1977)

M.A.Lone et al

Nucl Phys A277,413 (1977) C.R.Lux, N.T.Porile

Phys Lett B66,109 (1977) T.Tamura

et al

Verhandlungen der Deutschen Physikalischen Gesellschaft 6,1016(1977)

C. Alderliesten et al

INIS-MF-3329 p.393 (1976) 26. Conf Nucl Spect \& Nucl Structure, Baku, USSR, Feb 31976

B.A.Shilyaev et al

Nucl Phys A274, 168 (1976) G.L.Wales, R.C.Johnson

28th Conf on Nucl Spect \& Struct of the Atomic Nucleus, Alma-Ata,USSR,1978, INIS-MF-4388,p.277 V.E.Bunakov et al

Medical Physics 4, 486(1977) H.I.Amols et al direct reactions, neutron spectra

precompoundnucleus emission

inelastic scattering, direct reactions

inelastic scattering, quasi-free reactions

elastic scattering, recoils

pickup reactions nuclear cascades

energy spectra of Neutron Beams Produced by Protons and Deuterons of Various Energies Bombarding Beryllium and Lithium Targets of Various Thickness 
${ }^{9} \mathrm{Be}$

Cd

$\begin{array}{ll}208 \mathrm{~Pb} & \begin{array}{l}\text { Cross Sections and Reaction } \\ \text { Mechanisms of (p,pxn) Reacti- } \\ \text { ons on 208pb in the 24-52 MeV } \\ \text { Range }\end{array} \\ \mathrm{U} & \begin{array}{l}\text { Relative Mass Yields in Proton } \\ \text { Induced Fission on Natural } \\ \text { Uranium from Delayed Gamma } \\ \text { Rays Analysis }\end{array}\end{array}$

$181 \mathrm{Ta}$

75As

$90 \mathrm{Zr}$

$120 \mathrm{Sn}$

$208 \mathrm{~Pb}$

$48 \mathrm{Ca}$
Neutron Beams Generated by Protons on Beryllium

Excitation Functions of the $(p n)$ and $(p, 2 n)$ Reactions on Cd Isotopes

Measurement of Lithium, Beryllium and Boron Stable Isotope Production Cross Sections in Carbon-Spallation by Protons and Alpha Particles. Application to the Study of the Propagation of the Light Elements in Cosmic Rays

Cross Sections and Reaction 208 Range

Relative Mass Yields in Proton Uranium from Delayed Gamma Rays Analysis

Nature of a K X-Ray Satellite Peak as Determined from the Analysis of Fluorescence Spectra Induced by $20-50 \mathrm{MeV}$ Protons

Pre-Equilibrium Statistical Model: Comparison of Calculations with Two $(p, x n)$ Reactions

Preequilibrium Analysis of $(p, n)$ Spectra on Various Targets at Proton Energies of 25 to $45 \mathrm{MeV}$
Brit J of Radiol-

ogy (Eng) 49,587

(1976) S.W.Johnsen

et al

\section{Bull Acad Sci}

Phys Series

(USSR) 39,24(1975)

E.A.Skakun et al

Paris Univ. Orsay, Thesis, CSNSM-T-7502(1975)P.Fontes

Nucl Phys A262,52

(1976) H.Kawakami

et al

Revue Roumaine de Physique 21,69

(1976) A.Calboreanu

et al

J of Phys G2,L69

(1976) C.P.Randell

et al

$\mathrm{J}$ Inorg Nucl Chem

38, 1581(1976)

J.C.Brodovitch

et al

Nucl Phys A257, 15

(1976) M.Blann

et al reactions,

$\mathrm{x}$-radiation

neutron beams

charge-exchange reactions, knock-out reactions

spallation

knock-out reactions

delayed gamma radiation

precompoundnucleus emission

precompoundnucleus emission, gamma spectra 


\begin{tabular}{|c|c|}
\hline $\begin{array}{l}56 \mathrm{Fe} \\
103 \mathrm{Rh}\end{array}$ & $\begin{array}{l}\text { Generalized Exciton Model for } \\
\text { the Description of Preequili- } \\
\text { brium Angular Distributions }\end{array}$ \\
\hline $\begin{array}{l}12 \mathrm{C}, 6 \mathrm{Li} \\
9 \mathrm{Be}\end{array}$ & $\begin{array}{l}\text { Study of the (p,pn) Reaction } \\
\text { on 1p Shell Nuclei at } 46 \mathrm{MeV}\end{array}$ \\
\hline
\end{tabular}

$48 \mathrm{Ca}, 90 \mathrm{Zr}$

$120 \mathrm{Sn}$

$208 \mathrm{~Pb}$

$6,7 \mathrm{Li}$

${ }^{9} \mathrm{Be}, 12 \mathrm{C}$

$27 \mathrm{Al}$

103Rh

$159 \mathrm{~Tb}$

${ }^{169} \mathrm{Tm}$

$27 \mathrm{Al}, \mathrm{C}$

$\mathrm{Cu}$

$208 \mathrm{~Pb}$

$\mathrm{Be}, \mathrm{B}$

$12 \mathrm{C}$

$90 \mathrm{Zr}$

75 As

115 In
Analysis of the Preequilibrium Angular Distributions in the 45-MeV (p,n) Reaction

Production of Medium-Energy Neutrons from Proton Bombardment of Light Elements using Revised Neutron-Proton Differential Cross Sections

Comparison of Proton and Neutron Spectra. The Extended Griffin Model, Pairing and Isospin

Distributions of Secondary Particles Around Various Targets Exposed to $50 \mathrm{MeV}$ Protons

Pre-Equilibrium Model Evaluation of Neutron Spectra from Proton-Induced Reactions

Comparison of Measured Neutron Spectra with Predictions of an Intranuclear-Cascade Model

$\mathrm{Li}, \mathrm{Be}$, and $\mathrm{B}$ Production in Reactions of 45-100 MeV Protons with $12 \mathrm{C}$ : Astropliysical Implications

Charged-Particle Emission in Reactions of $90 \mathrm{Zr}$ with $10-86$ MeV Protons

Excitation Functions and Isomeric Yield Ratios of ( $p, x n)$ Reactions Induced in $75 \mathrm{As}$ and and 115 In by $20-85 \mathrm{MeV}$ Protons
Zeit fur Physik

A276, 145(1976)

G.Mantzouranis et al

Manitoba Univ, Thesis, CTOM-20405 (1974) C.A.Miller

Phys Rev C14,2018

(1976)

GManzouranis

Nucl Instr Meth

134,537(1976)

J.L.Romero et al

Zeit fur Physik

A275, 175(1975)

C.Kalbach et al

Nucl Instr Meth

133,213(1976)

A.Fasso, M.Hoefert

Phys Rev C14,573

(1976) E.Gadioli

et al

Phys Rev Cl4,748

(1976) A.Galonsky

et al

Phys Rev C14,410

1976) C.T.Roche

et al

Phys Rev $\underline{C 14}, 64$

(1976) M.V.Kantelo, JJ.Hogan

McGill Univ

(Canada) Thesis

CTOM-18162(1973)

J.C.Brodovitch precompoundnucleus emission, neutron spectra

knock-out reactions

precompoundnucleus emission

neutron sources

precompoundnucleus emission

spallation

charge-exchange reactions, precompoundnucleus emission, neutron spectra

Monte Carlo method, neutron spectra, nuclear cascades

spallation

precompoundnucleus emission

Monte Carlo method 
$12 \mathrm{C}$

$58 \mathrm{Ni}$

$208 \mathrm{pb}$

$40 \mathrm{Ca}$

$208 \mathrm{~Pb}$

160

${ }^{181} \mathrm{Ta}$

$115 \mathrm{Sn}$

181W

$238 \mathrm{u}$
Optical Model Analysis of Proton-Carbon Elastic Scattering in the Range 20 to $50 \mathrm{MeV}$

Charged Particle Spectra from $100 \mathrm{MeV}$ Protons on $58 \mathrm{Ni}$

Alpha-Emission by Precompound Mechanism in Heavy Nuclei

Quasi-Free Scattering Model for Nuclear Reactions in the Continuum

Cross Sections of (p,pxn) Reactions on $2 \cup 8 \mathrm{~Pb}$

Proton Total Reaction Cross Sections for the Doubly Magic Nuclei $160,40 \mathrm{Ca}$, and $208 \mathrm{~Pb}$ in the Energy Range $20-50 \mathrm{MeV}$

Angular Distribution of Nucleons in Nucleon-Induced Preequilibrium Reactions

Proton and Deuteron Fission of 238U

Radiation Heating of Materials During Irradiation with Charged Particles
Zeit fur Phys

A274,339(1975)

R.Sprickmann et al

Univ of Maryland report ORO-485626 p.360(1975)

J.R.Wu et al

Julich report JUL-CONF-16,V1,p. 85(1975) Conf on Highly Excited States of Nuclei L.Milazzo-Colli et al

U of Rochester, N.Y.Thesis(1975)

A.L.C.Mignerey

Tokoyo Univ report NP-20526 p.54(1974)

J.ORasmussen et al

Phys Rev C12,1167 (1975) R.F.Carlson et al

Phys Lett B57,220

(1975)

G.Mantzouranis et al

Egypt J Chem, Spec Issue pp.1-7(1974)

A.T.Kandil, G.R.Choppin

Izv Akad Nauk Kaz SSR 6,84(1974)

S.S.Ibragimov et al elastic

scattering

energy spectra, precompoundnucleus emission

heavy nuclei, precompoundnucleus emission

heavy nuclei, intermediate mass, quasifree reactions

compoundnucleus reactions optical models

angular' ästribution, heavy nuclei, intermediate mass nuclei,precompound nucleus emission

fission

radiation heating 
Energy Dependence of Proton

Damage in Silicon

COMPD: A Computer Program for the Calculation of the Excitation Functions by Means of Monte Carlo Method

A Technique for Measuring Proton Total Reaction Cross Sections at Medium Energies

Energy Distributions of Charged and Neutral Hydrogen Atoms Backscattered from Metal Surfaces Bombarded with 5 to $18 \mathrm{MeV}$ Protons

$27 \mathrm{Al}, 12 \mathrm{C} \quad$ Optical Model Analysis of Proton and Deuteron Scattering and DWBA Calculations on (p,d)Reactions at $185 \mathrm{MeV}$

${ }^{209} \mathrm{Bi}$ $12 \mathrm{C}, 54 \mathrm{Fe}$

$27 \mathrm{Al},{ }^{9} \mathrm{Be}$ C

Al

${ }^{56} \mathrm{Fe},{ }^{58} \mathrm{Ni}$
Comparisons of Predictions from Two Intranuclear-Cascade Models with Measured Secondary Proton Spectra at Several Angles from 62- and 39-MeV Protons on Various Elements

Measurements of the Proton Total Reaction Cross Section for Light Nuclei Between 20 and $48 \mathrm{MeV}$

Effect of High-Energy Protons on Mechanical Properties of Aluminum

Gamma Rays Observed from 100 $\mathrm{MeV}$ Protons Interacting with $56 \mathrm{Fe}$ and $58 \mathrm{Ni}$
Izv Akad Nauk Kaz SSR 6,50(1974)

A.F.Akkerman et al

Tokoyo Univ report

INS-TCH-10(1974)

K.Komura, S.Tanaka

Nucl Instr Meth

123,509(1975)

R.F.Carlson et al

Max Planck Instit. report IPP-9/18 (1975) P.Meischner, H.Verbeek

Phys Scripta 10, 159 (1974)

A.Ingemarsson, G.Tibell

Phys Rev C10,2472 (1974) H.W.Bertini et al

Phys Rev C10,2237 (1974) W.F.McGill

Fiz Khim Obrab Mater 5, 151(1974) G.G.Bondarenko, L.I.Ivanov

Phys Rev Lett 33 , 1493(1974) C.C.Chang et al elastic scattering, physical radiation effects

compoundreactions, evaporation model, Monte Carlo method

elastic scattering

radiation effects

optical models, pickup reactions

energy spectra, inelastic scattering, nuclear cascades, precompoundnucleus emission

total cross sections

dislocations, physical radiation

precompoundnucleus emission 
${ }^{209} \mathrm{Bi}$

$181 \mathrm{Ta}$

W

$208 \mathrm{~Pb}$

$209 \mathrm{Bi}$

$12 \mathrm{C}$

${ }^{59} \mathrm{Co},{ }^{63} \mathrm{Cu}$

$56 \mathrm{Fe}$

$181 \mathrm{Ta}$

$209 \mathrm{Bi}$

${ }^{54} \mathrm{Fe}$

6Li, $12 \mathrm{C}$

$9 \mathrm{Be}$
Reactions on $209 \mathrm{Bi}$ Induced by

Intermediate Energy Protons

and the Effect of Direct

Reactions

Monte Carlo Programs for

Calculating Three-Dimensional

High-Energy ( $50 \mathrm{MeV}-500 \mathrm{GeV}$ )

Hadron Cascades in Matter

Analysis of (particle,xn) Reactions on Tantalum and Gold. I.

181 Ta(alpha, xn) Excitation

Functions Up to $104 \mathrm{MeV}$ and

Equilibrium Statistical Model

Analysis

Periodic Dependence of Gaussian

Electronic Stopping Cross

Section with Energetic Ions in

Solid Targets

Effects of Irradiation-Induced

Defects on the Mechanical

Properties of Tungsten

Optical-Model Analysis of $p+$ $208 \mathrm{~Pb}$ Elastic Scattering from

$15-1000 \mathrm{MeV}$

Comparisons of Predictions from

Two Intranuclear-Cascade Models

with Measured Secondary Proton

Spectra at Several Angles from

62- and 39-MeV Protons on

Various Elements

De-Excitation by Statistical

Pre-Equilibrium Alpha

Emission

A-Priori Pre-Equilibrium Decay

Models

(p,pn) Reactions on Light

Nuclei at $46 \mathrm{MeV}$
Nucl Phys A230,98

(1974) K.Miyano

et al

Compui Phys Commun

7,327(1974)

J.Ranft, J,T,Routti

Nucl Phys A228, 165

(1974) F.Hermes

et al

Jap J Appl Phys

13,393(1974)

K.Kanaya, K.Hojou

U of Pittsburgh

Thesis(1973)

C.S.Reft

Phys Rev $\underline{C} 10,307$

(1974)

W.T.H.Van Ders et al

Oak Ridge report

ORNL-TM-4638(1974)

H.W.Bertini et al

Univ Lyon report

LYCEN-7347(1973)

Thesis, N.Chevarier

Nukleonika 19,183

(1974) M.Blann

Phys Rev Lett 32 ,

684(1974)

C.A. Miller et al direct reactions, precompoundnucleus emission

charged-particle transport, Monte Carlo method, neutron transport

gamma spectra, Monte Carlo method

charged-particle transport, electron-ion collisions, stopping power

dislocations, radiation effects

optical models

nuclear cascades, quasi-elastic scattering

nuclear cascades, precompoundnucleus emission

nucleon-nucleon interactions, precompoundnucleus emission

knock-out reactions, quasi-elastic scattering 
Table I (Cont.)

$\mathrm{Cu}$

$45 \mathrm{Sc}$

$7 \mathrm{Li}$

209Bi

$54 \mathrm{Fe}$

$\mathrm{Cu}$

$\mathrm{H}$

$\mathrm{Bi}$

${ }^{197} \mathrm{Au}$

$170 \mathrm{Hf}$

${ }^{181} \mathrm{Ta}$

$168 \mathrm{Yb}$
Shielding from Neutrons Generated in Targets by a Beam of $1 \mathrm{GeV}$ Protons

Charged Particles Produced in the Bombardment of $45 \mathrm{Sc}$ by 45 MeV Prolons

Fast Forward Neutron Production in the $7 \mathrm{Li}(\mathrm{p}, \mathrm{n}) 7 \mathrm{Be}$ Reaction for 41 - and $64 \mathrm{MeV}$ Protons

A Priori Pre-Equilibrium Decay Models

Complete Hydrogen and Helium Particle Spectra from 30- to 60-MeV Proton Bombardment of Nuclei with $A=12$ to 209 and Comparison with the Intranuclear Cascade Model

Spallation of Copper by 65-MeV $\mathrm{Pi}^{+}$and $\mathrm{Pi}-$ and by $205-\mathrm{MeV}$ Protons

Gold L X-Ray Production by 0.5 to $30-\mathrm{MeV}$ Protons

X-Ray Ëmission Following Nuclear Reactions

De-Excitation of Even-Even Isotopes of $\mathrm{Yb}, \mathrm{Hf}$, and $\mathrm{W}$ Produced in ( $p, x n$ gamma) Reactions
Tr Radiotekh Inst 9,117(1972)

B.V.Man'ko, B.S.Sychev

U of S Calif
Thesis(1973)
H.Spitzer

Nucl Instr Meth

113,185(1973)

J.W.Wachter et al

Nucl Phys A213,570

(1973) M.Blann

Phys Rev C8, 1045

(1973)

F.E.Bertrand,

R.W.Peele

Phys Rev C8,594

(1973) C.K.Garrett,

A.L.Turkevich

Phys Rev A7,566

(1973)

S.M.Shafroth et al

Phys Rev Lett 30 ,

863(1973)

G.Deconninck,

N.Longequeue

J Phys (Paris) 34, 345(1973)

C.Birattari et al shielding

reaction

mechanisms,

DWBA, compound-

nucleus reactions,pickup reactions

charge-exchange reactions, neutron spectra

nucleon-nucleon potential, optical models

heavy nuclei, light nuclei, nuclear cascades

nuclear cascacics

emission spectra, hydrogen ions, $x$-ray spectra

x-ray spectra

compound nucleus reactions, gamma radiation 
$7 \mathrm{Li}$

$160 \mathrm{Gd}$

${ }^{169} \mathrm{Tm}$

$12 \mathrm{C}$

$58,60 \mathrm{Ni}$

C. $27 \mathrm{Al}$

${ }^{59} \mathrm{Co}$

$\mathrm{Cu}$

${ }^{54} \mathrm{Fe}$

$24 \mathrm{Mg}$

${ }^{28} \mathrm{Si}$
High-Energy Neutrons Produced by $740-\mathrm{MeV}$ Protons on Uranium

Characteristic L X-Ray Spectra irom Proton, Alpha-Particle, and Oxygen: Bombardment of Sn

Fast Forward Neutron Production in the ${ }^{7} \mathrm{Li}(p, n){ }^{7} \mathrm{Be}$ Reaction for 41- and 64-MeV Protons

Reaction Mechanisms in (p, $\left.p^{\prime}\right)$ Reactions Exciting the HighEnergy Continuum

Pre-Equilibrium Process in $(p, n)$ Reactions

Elastic Scattering of 46-MeV Protons by $12 \mathrm{C}, 58 \mathrm{Ni}$, and $60 \mathrm{Ni}$

Energy and Angle Distributions of Neutrons Emitted in the Interactions of $3 \mathrm{GeV}$ Protons with Carbon, Aluminum, and Cobalt

Thin-Target Cross Sections for Some $\mathrm{Cr}, \mathrm{Mn}, \mathrm{Fe}, \mathrm{Co}, \mathrm{Ni}$, and $\mathrm{Zn}$ Nuclides Produced in Copper by $82-416-\mathrm{MeV}$ Protons

Extensions to the PreEquilibrium Statistical Model and a Study of Complex Particle Emission

Optical-Model Analysis of the Elastic Scattering of $100 \mathrm{MeV}$ Protons from $24 \mathrm{Mg}$ and $28 \mathrm{si}$

Properties of Proton-Irradiated Diamond
Kent State Univ report APS Meeting COO-2231-4(1972)

Wash DC, R.Madey, F.M.Waterman

Phys Rev A7,1244 (19/3) D.K.Olsen

et al

Oak Ridge report ORNL-TM-3853(1973)

J.W.Wachter et al

Phys Rev C7,331 (1973) B.L.Cohen et al

Nucl Phys A201,579 (1973) C.Birattari et al

Particles 3 Nuclei 4,192(1972)

H.Willmes et al

Thesis(1972)

Univ. Microfilms

\#72-25,638

W.J.Nalesnik

Phys Rev C6, 1235

(1972)

H.R.Heydegger et al

Nucl Phys $\$ 193,417$

(1972) C.K.Cline

Nucl Phys A193,438

(1972) Y.S.Horowitz

Radiation Effects

14,245(1972)

K.P.Ananthanarayanan

et al knock-out

reactions, neutron spectra

ion collisions, $x$-ray spectra

neutron spectra

DWBA analysis

pre-equilibrium mechanisms

elastic

scattering

$10-900 \mathrm{MeV}$

neutron energy,

angular

distributions

thin-target

cross sections

pre-equilibrium statistical model

elastic scattering

diamonds, infrared spectra 
Table I (Cont.)

$58,60 \mathrm{Ni}$

$61,62 \mathrm{Ni}$

96Mo

$6 \mathrm{Li}$

$\mathrm{Xe}$

methane

$89 Y$

$160,12 \mathrm{C}$

$14 \mathrm{~N}$

$27 \mathrm{AJ}, \mathrm{O}$

$\mathrm{Mg}, \mathrm{Si}$

$\mathrm{Cl}$

$\mathrm{C}, \mathrm{N}, \mathrm{O}$

$141 P_{T}$

$6 \mathrm{Li}$

$9^{\mathrm{Be}}$

$6,7 \mathrm{Li}$
Nuclear Reactions of Nickel

with Protons up to $56 \mathrm{MeV}$

Excitation Function of (p,pn)

Reactions at High Energy

96Mo(p,xn) Reaction from 10 to $80 \mathrm{MeV}$

Elastic and inelastic Scattering of Protons from 6 $\mathrm{Li}$

Between 25 and $45 \mathrm{MeV}$

Fluctuations in the Energy

Loss of 66- and 100-MeV Protons

in a Thin Proportional Counter

Calculations of Isomer Ratios

Based on the Statistical and

Cascade-Evaporation Theories

Reaction Cross Sections for $30-$

to $60-\mathrm{MeV}$ Protons on Various

Elements: Comparison of Theoretical Results with Experiment

Investigation of (p,pd) Reaction on Light Nuclei at Energy $50 \mathrm{MeV}$

Spallation Reactions Produced by 50,100 , and $153 \mathrm{MeV}$ Protons with Light Nuclei

Reaction (p,2p) on Light Nuclei at $50 \mathrm{MeV}$

Study of the $141 \operatorname{Pr}(p, x n)$ Reaction from 10 to $85 \mathrm{MeV}$

6Li(p,pd) ${ }^{4} \mathrm{He}$ Reaction at 100 $\mathrm{MeV}$

Inelastic Scattering of $50 \mathrm{MeV}$

Protons by $6 \mathrm{Li},{ }^{7} \mathrm{Li}$, and ${ }^{9} \mathrm{Be}$
I Inorg Nucl Chem

34.2419(1972)

S.Tanaka et al

Nucl Phys A190,501

(1972) S.Das et al

Phys Rev C6,810

(1972) J.J.Hogan

Nucl Phys A189,35

(1972) K.H.Bray

et al

Phys Rev A6,250

(1972) G.T.Huetter

et al

Nucl Phys A188,409

(1972) G.B.Saha,

L.Yaffe

Phys Rev CS, 2118

(1972) H.W.Bertini

Yaciern Fiz 15,425

(1972) V.V.Boikov

et al

Compt Rend B273,

1054(1971)

R. Bimbot, H.Gauvin

Yadern Fiz 14,676

(1971) I.I.Losh-

chakov et al

J Inorg Nucl Chem

33,3627(1971)

J.J.Hogan

Nucl Phys A178,225

(1971)

I.A.Mackenzie

et al

Nucl Phys A172,166

(1971) G.S.Mani

et al excitation

functions

excitation

functions, knock-

out reactions

nuclear cascades, intranuclear cascade models

elastic, inelastic scattering

proton energy

losses

nuclear cascades

$\therefore$ sanuclear cascade model

recoil angular distributions and momenta for

residual nuclei production

impulse approximation

intranuclear cascade

impulse approximation, quasi-elastic scattering

inelastic scattering 


\begin{tabular}{|c|c|c|c|}
\hline $\begin{array}{l}9 \mathrm{Be}, \mathrm{B} \\
\mathrm{N}\end{array}$ & $\begin{array}{l}\text { Production of } 7 \mathrm{Be} \text { from Light } \\
\text { Targets by } 30 \mathrm{MeV} \text { to } 24 \mathrm{GeV} \\
\text { Protons }\end{array}$ & $\begin{array}{l}\text { J Inorg Nucl Chem } \\
\text { 33,2720(1971) } \\
\text { M.Barbier, S.Regnier }\end{array}$ & \\
\hline $\mathrm{Fe}$ & $\begin{array}{l}\text { High-Energy Proton Spallation } \\
\text { of Iron }\end{array}$ & $\begin{array}{l}\text { Phys Rev C4,1257 } \\
\text { (1971) } \\
\text { R.L.Brodzinski et al }\end{array}$ & spallation \\
\hline \multirow[t]{2}{*}{$\mathrm{Ti}$} & $\begin{array}{l}\text { High-Energy Proton Spallation } \\
\text { of Titanium }\end{array}$ & $\begin{array}{l}\text { Phys Rev C4,1250 } \\
\text { (1971) } \\
\text { R.L.Brodzinski et al }\end{array}$ & spallation \\
\hline & $\begin{array}{l}\text { Total-Reaction-Cross-Section } \\
\text { Measurements for } 30-60-\mathrm{MeV} \\
\text { Protons and the Imaginary } \\
\text { Optical Potential }\end{array}$ & $\begin{array}{l}\text { Phys Rev C4, } 1114 \\
\text { (1971) J.J.Menet } \\
\text { et al }\end{array}$ & $\begin{array}{l}\text { optical model, } \\
\text { total cross } \\
\text { sections }\end{array}$ \\
\hline $181 \mathrm{Ta}$ & $\begin{array}{l}\text { Precompound Decay from a Time- } \\
\text { Dependent Point of View }\end{array}$ & $\begin{array}{l}\text { Phys Rev C3, } 1847 \\
\text { (1971) G.D.Harp, } \\
\text { J.M.Miller }\end{array}$ & $\begin{array}{l}\text { time-dependent } \\
\text { model }\end{array}$ \\
\hline $27 \mathrm{Al}, \mathrm{Si}$ & $\begin{array}{l}\text { Energy Dependence of Proton } \\
\text { Elastic Scattering Cross } \\
\text { Sections from } \mathrm{Al} \text { and Si }\end{array}$ & $\begin{array}{l}\text { Nucl Phys A169,600 } \\
\text { (1971) H.S.Sandhu } \\
\text { et al }\end{array}$ & $\begin{array}{l}\text { optic al model } \\
\text { parameters }\end{array}$ \\
\hline \multirow[t]{2}{*}{$\begin{array}{l}27 \mathrm{Al} \\
9 \mathrm{Be}, 12 \mathrm{C}\end{array}$} & $\begin{array}{l}\text { Production of Medium-Energy } \\
\text { Neutrons from Proton Bombard- } \\
\text { ment of Light Elements . }\end{array}$ & $\begin{array}{l}\text { Nucl Instr Meth } \\
\text { 94,421(1971) } \\
\text { J.A.Jungerman }\end{array}$ & spectra \\
\hline & $\begin{array}{l}\text { Electron Capture and Loss by } \\
\text { Atoms, Protons, and Negative } \\
\text { Hydrogen Ions in Collisions } \\
\text { with Atoms and Molecules }\end{array}$ & $\begin{array}{l}\text { Sov Phys } 15,1947 \\
\text { (1971) } \\
\text { N.V.Fedorenko }\end{array}$ & $\begin{array}{l}\text { hydrogen ions, } \\
\text { reactions with } \\
\text { gases }\end{array}$ \\
\hline $\begin{array}{l}{ }^{209} \mathrm{Bi} \\
181 \mathrm{Ta} \\
209 \mathrm{Bi}\end{array}$ & $\begin{array}{l}\text { (p,xn) Reactions Induced in } \\
169 \mathrm{Tm}, 181 \mathrm{Ta} \text {, and } 209 \mathrm{Bi} \\
\text { with } 20 \text { to } 45 \mathrm{MeV} \text { Protons }\end{array}$ & $\begin{array}{l}\text { Nucl Phys A166,605 } \\
\text { (1971) } \\
\text { C.Birattari et al }\end{array}$ & $\begin{array}{l}\text { precompound and } \\
\text { statistical con- } \\
\text { tributions }\end{array}$ \\
\hline $40 \mathrm{Ca}$ & $\begin{array}{l}\text { Optical-Model Analysis of } p+ \\
40 \text { Ca Elastic Scattering from } \\
10 \text { to } 180 \mathrm{MeV}\end{array}$ & $\begin{array}{l}\text { Phys Rev C3,1550 } \\
(1971) \\
\text { W.T.H.Van Oers }\end{array}$ & optical model \\
\hline $\begin{array}{l}232 \mathrm{Th} \\
23 \% \mathrm{U} \\
237 \mathrm{~Np}\end{array}$ & $\begin{array}{l}\text { Phenomena of Fission of Heavy } \\
\text { Nuclei by High-Energy Protons }\end{array}$ & $\begin{array}{l}\text { Acta Phys Austr } 29 \text {, } \\
\text { 174(1969) H.Axmann, } \\
\text { M.Barbier }\end{array}$ & fission \\
\hline 208 ph & $\begin{array}{l}\text { Elastic Scattering of } 50 \mathrm{MeV} \\
\text { Protons by Piuclei in the Range } \\
\text { from } 42 \mathrm{Ca} \text { to } 208 \mathrm{~Pb}\end{array}$ & $\begin{array}{l}\text { Nucl Phys A165,384 } \\
\text { (1971) G.S.Mani } \\
\text { et al }\end{array}$ & $\begin{array}{l}\text { scattering by } \\
\text { nineteen nuclei, } \\
\text { optical model } \\
\text { parameters }\end{array}$ \\
\hline
\end{tabular}


$6,7 \mathrm{Li}$

$9 \mathrm{Be}$

$69,71 \mathrm{Ga}$

$89 \mathrm{Y}, 55 \mathrm{Mn}$

$59 \mathrm{Co}$

$68 \mathrm{Zn}$

$45 \mathrm{Sc}$

$\mathrm{N}, \mathrm{C}, \mathrm{O}$

$\mathrm{Si},{ }^{9} \mathrm{Be}$

$27 \mathrm{Al}$

$\mathrm{Ca}, \mathrm{V}$

$27 \mathrm{Al}$

$12 \mathrm{C}$

$56 \mathrm{Fe}$

$120 \mathrm{Sn}$

$56 \mathrm{Fe}$

$208 \mathrm{~Pb}$

$56 \mathrm{Fe}$

$27 \mathrm{Al}, 14 \mathrm{~N}$
Elastic Scattering of $50 \mathrm{MeV}$

Protons by Light Nuclei

Estimation of Unknown

Excitation Functions

for (alpha, xn)-,

(d,xn)- and (d,pxn)-,

and $(p, x n)$-Reactions

Cross Sections for the Pto-

duction of Nuclides from Med-

ium Weight Elements by High-

Energy Proton Bombardment

Nuclear Interactions of $45 \mathrm{Sc}$

and $68 \mathrm{Zn}$ with Protons of

Medium Energy

Backward Angle Scattering of 50-MeV Protons from C, O, N, and $\mathrm{Be}$

Proton Total Reaction Cross

Sections for Light Nuclei

Between 24 and $46 \mathrm{MeV}$

X-Ray Spectra from Oxygen-Ion Bombardments on $\mathrm{Ca}$ and $\mathrm{V}$ at 15 $\mathrm{MeV}$

Calculation of the Photon-

Production Spectrum from

Proton-Nucleus Collisions in

the Energy Range 15 to 150

$\mathrm{MeV}$ and Comparison with

Experiment

Tabulated Cross Sections For Hydrogen and Helium Particles Produced by $62-$ and $29-\mathrm{MeV}$

Protons on 120Sn

Excitation Functions for the Bombardment of $56 \mathrm{Fe}$ with Protons

Calculation of the Neutron Spectra from Proton-Nucleus Nonelastic Collisions in the Energy Range 15 to $18 \mathrm{MeV}$ and Comparison with Experiment
Nucl Phys A165, 145

optical model

1971) G.J.Mani et al

Oak Ridge report

ORNL-TR-3020(ND)

J.Lange, H.Muenzel

Nucl Phys A155,49

(1970) B.K.Gupta

et al

Nucl Phys Al50,11

(1970) T.McGee et al

Nucl Phys A157,145

(1970) N.M.Clark

et al

Thesis(1969)

(Univ unknown)

Univ Microfilm order

70-8247 W.F.McGill

Phys Rev Lett 25 ,

983(1970) D.Burch,

P.Richard

Nucl Sci Eng 41,47

(1970) Y.Shima,

R.G.Alsmiller Jr

gamma radiation,

Oak Ridge report

ORNL-4471(1970)

F.E.Bertrand,

R.W.Peelle

J Inorg Nucl Chem

32,1419(1970)

I.L.Jenkins, A.G.Wain

Nucl Sci Eng 40,

254(1970)

R.G.Alsmiller,

O.W Hermann intranuclear

cascade

excitation

functions,

thick-target

irradiation

spallation

products

slastic and

inelastic

cross sections

total cross

sections

atomic models, $\mathrm{x}$-ray spectra

charged-particle production data

excitation functions

intranuclear

cascade 


\begin{tabular}{|c|c|}
\hline $12 \mathrm{C}$ & $\begin{array}{l}\text { Inelastic Scattering of } 100 \mathrm{MeV} \\
\text { Protons from } 12 \mathrm{C} \text { Using a Ge(Li) } \\
\text { Totai-Absorption Proton Counter }\end{array}$ \\
\hline${ }^{54} \mathrm{Fe}$ & $\begin{array}{l}\text { Tabulated Cross Sections for } \\
\text { Hydrogen and Helium Particles } \\
\text { Produced by } 62-, 39-\text {, and } 29- \\
\text { MeV Protons on } 54 \mathrm{Fe}\end{array}$ \\
\hline $\begin{array}{l}A r, \mathbb{N} \\
0\end{array}$ & $\begin{array}{l}\text { Electro-Caphure Cross-Sections } \\
\text { in Various Gases by Piotons } \\
\text { with Energies from } 25 \text { to } 38 \mathrm{MeV}\end{array}$ \\
\hline $197 \mathrm{Au}$ & $\begin{array}{l}\text { Tabulated Cross Sections for } \\
\text { Hydrogen and Helium Particles } \\
\text { Produced by } 62-\text { and } 29-\mathrm{MeV} \\
\text { Protons on } 197 \mathrm{Au}\end{array}$ \\
\hline
\end{tabular}

$27 \mathrm{Al}$

${ }^{89} \mathrm{Y}$

$9_{\mathrm{Be}}$

$10,11 \mathrm{~B}$

$12,13 \mathrm{C}$

$56 \mathrm{Fe}$
$27 \mathrm{Al}, \mathrm{Fe}, \mathrm{C}$
$\mathrm{Pb}, \mathrm{Cu}, \mathrm{U}$
$\mathrm{Ti}, 9 \mathrm{Be}$
$\mathrm{Cd}$

Proton Optical Potentials from 30 to $180 \mathrm{MeV}$

Tabulated Cross Sections for Hydrogen and Helium Particles

Producer by $62-\mathrm{MeV}$ Protons on $89 \mathrm{Y}$

Optical-Model Analysis of

Nucleon Scattering from $1 p$-Shell

Nuclei Between 10 and $50 \mathrm{MeV}$

Tabulated Cross Sections for Hydrogen and Helium Particles Produced by $61-\mathrm{MeV}$ Protons on $56 \mathrm{Fe}$

Energy Dependence of Interaction Cross Sections of Nucleons with Atomic Nuclei at Energies Higher than $50 \mathrm{MeV}$
Can J Phys $\underline{48,201}$

(1970)

Y.S.Horowitz, R.E.Bell

Oak Ridge report

ORNL-4469(1970)

F.E.Bertrand,

R.W.Peelle

Nuovo Cim A64, 1068

(1969) E.Acerbi

et al

Oak Ridge report

ORNL-4460(1969)

F.E.Bertrand,

R.WPeelle

Oak Ridge report

ORNL-4455(1969)

F.E.Bertrand,

R.W Peelle

Nucl Phys A138,61

Total Reaction Cross Section Measurements with 60-MeV Protons
(1969) K.Seth

Oak Ridge report

ORNL-4450;1969)

F.E.Bertrand,

R.W.Peelle

Phys Rev 182,977

(1969) B.A.Watson

et al

Oak Ridge report

ORNL-4456(1969)

F.E.Bertrand,

R.W.Peelle

Oak Ridge report

ORNL-TR-2148(ND)

V.S.Barashenkov

et al

Phys Rev Lett 22 ,

1128(1969)

J J.H.Menet et al inelastic

scattering

charged-particle spectra

electron-capture

differential cross sections, hydrogen and helium isotope production

differential cross sections for hydrogen and helium isotope production

optical model

differential cross sections

optical model

production cross sections for hydrogen and helium

energy dependence of cross sections

ten nuclei, carbon - lead 
Table I (Cont.)

$59 \mathrm{Co}$

${ }^{63} \mathrm{Cu}$

$12 \mathrm{C}$

$27 \mathrm{Al}, 40 \mathrm{Ca}$

${ }^{209} \mathrm{Bi}, 12 \mathrm{C}$

$27 \mathrm{Al}, 56 \mathrm{Fe}$

160

$\mathrm{Cu}$

$\mathrm{G} s, \mathrm{Si}$

${ }^{12 C}$

$238 \mathrm{U}$
Simulation of the Interaction of Cosmic Rays with the Lunar Surface by Proton Bombardment of a 'Thick Target'. Pt.1.

Reactions of Low Energy Neutrons

Microscopic Effective Interaction for $155-\mathrm{MeV}$ Protons

Gamma Rays from Proton

Bombarument of Nuclei

Elastic and Inelastic ProtonNucleus Scattering at $156 \mathrm{MeV}$ : Experimental Study and Analysis in Impulse Approximation

Multinucleon Removal Induced by High-Energy Protons

Calculated Neutron Spectrum from $800-\mathrm{MeV}$ Protons Incident on a Copper Beam Stop

Comparison of Radiation Damage in Si and Ge Irradiated with $660 \mathrm{MeV}$ Protons and Fast Reactor Neutrons

Specialized Reactions: Reactions at Intermediate Energies: Baryon-Nucleus Collisions. 150 MeV-1GeV

Spectrum and Shielding Measurements and Calculations of Neutrons Produced by $800 \mathrm{MeV}$ Protons

Multi-Step Processes in HighEnergy Elastic and Inelastic Nuclear Scattering

Electronuclear Method of Neutron Production

Extension of the Isobar Model for Intranuclear Cascades to $1 \mathrm{GeV}$
Radiochim Acta 21 , 169(1974) R_Michel

et al

Phys Rev C13,444

(1976) M.Buenerd

Univ of Maryland report ORC 4856-26 p.348, O.Artun et al

IPNO-T-75-01(1975)

Univ of Paris

Thesis, V.Comparat

Phys Rev Lett 35 , 773(1975) O.Artun et al

Los Alamos report

LA-6043-MS(1975)

D.G.Perry

Sov Phys Semicond 9,352(1975)

S.R.Novikov,

E.E.Rubinova

Nucl Spect and

Reactions, Pt. B

Academie Press

(1974) G.Igo

Los Alamos report

LA-UR-75-34 I(1975)

L.R. Veeser et al

Nucl Phys A236,423

(19/4) E.Kujawski

At Energ(USSR) 37 , 475(1974)

V.S.Barashenkov

et al

Phys Rev C10,2387

(1974) G.D.Harp epithermal

neutrons

inelastic

scattering

direct reactions, gamma radiation, light nuclei

impulse approximation, inelastic scattering

gamma radiation

neutron spectra

physical radiation recoils

light nuclei, quasi-elastic scattering

gypsum, neutron spectra, spallation

inelastic scattering

neutron spectra, nuclear cascades

energy spectra, nuclear cascades, spallation 
$\mathrm{Ni}$

${ }^{9} \mathrm{Be}, \mathrm{Cu}$

U

$\mathrm{U}$

$40 \mathrm{Ca}, 27 \mathrm{Nl}$

$209 \mathrm{Bi}, 56 \mathrm{Fe}$

$62 \mathrm{Ni}$
Neutrons Produced by $740-\mathrm{MeV}$

Protons on Uranium

Properties of Light Transition

Radiation of $660 \mathrm{MeV}$ Proton

(gamma = 1.7) Inclined Inci-

dence on a Thick Nickel Plate

Neutron Spectra at 00 from 724 -

$\mathrm{MeV}$ Protons on $\mathrm{Be}$ and $\mathrm{Cu}$

Mass Distribution in Fission of

Uranium Induced by $170-\mathrm{MeV}$

Protons

Calculation of Electronuclear

Method for Neutron Production

Optical Model Partial Wave

Analysis of Intermediate Energy

( 0.6 to $1.0 \mathrm{GeV}$ ) Proton-Nucleus

Elastic Scattering

Inelastic Interactions of High

Energy Nucleons with Heavy

Nuclei

Proton-Nucleus Elastic Scat-

tering at $156 \mathrm{MeV}$

Direct Interaction of High

Energy Particles with Nuclei

Invariant Effects in Backward Emission of $p, d, t$ by Nuclei Bombarded by 0.7 to $6 \mathrm{GeV}$

Elementary Particles

$\mathrm{Li}^{58} \mathrm{Ni}$
Investigation of Isotopic Effects in Nuclear Reactions Induced by $660-\mathrm{MeV}$ Protons
Nucl Instr Meth

117,509(1974)

L.R.Veeser et al

Dubna report

JINR.P1-7956(1974)

V.P.Zrelov et al

Kent State Univ report COO-2231-1

(1973) R.Madey,

F.M.Waterman

J Inorg Nucl Chem

36,245(1974)

I.Haldorsen et al

Dubna report

JINR-P2-7694(1974)

V.S.Barashenkov et al

Wayne State Univ

Thesis(1973)

B.C.Clark

Nucl Phys A222,204

(1974)

V.S.Barashenkov et al

Nucl Phys A221,403

(1974) V.Comparat

et al

Atomizdat(1972)

F.B.Denisov,

V.N.Makhedov

Yadem Fi- 19,1266

(1974)

Y.Bayukov et al

Yadern Fiz 19,32

(1974) V.I.Bogatin

et al neutron spectra

energy spectra

nuclear cascades

fission yield

neutron spectra

nuclear cascades, spallation

optical-model

analysis

Goldberger model, impulse approximation

particle production, spectra

spallation 
U

$\begin{array}{ll}9 \mathrm{Be} & \begin{array}{l}\text { Neutron Spectra at } 00 \text { from } 724 \\ \mathrm{Cu}\end{array} \\ \mathrm{Cs} & \begin{array}{l}\text { Spectra of Radioactivity } \\ \text { Induced in Cesium Iodide } \\ \text { Scintillator Crystals by } \\ 155 \mathrm{MeV} \text { Protons }\end{array} \\ \mathrm{Ta} & \begin{array}{l}\text { Calculations of Spallation } \\ \text { Yields of Products Induced by } \\ \text { the Interaction of High-Energy } \\ \text { Protons with Complex Nuclei }\end{array}\end{array}$

$\mathrm{Be}, \mathrm{C}, \mathrm{Li}$

$238 \mathrm{U}$

${ }^{209} \mathrm{Bi}$

${ }^{197} \mathrm{Au}$

238U
Systematics in the $(p, x n)$ and (p,pxn) Reaction Cross Sections

High-Energy Neutrons Produced by $740-\mathrm{MeV}$ Protons on Uranium

Energy Dependence of the Cross Section of Fast Deuteron Knock-Out from $\mathrm{Li}, \mathrm{Be}$, and $\mathrm{C}$ by 380 to $665 \mathrm{MeV}$ Protons

Flures of Neutrons Generated by High Energy Protons in Thick Blocks of Uranium

Cluster Structure and Inelastic Interactions of Fast Protons with Light Nuclei

Inelastic Interactions of High Energy Nucleons with Heavy Nuclei

Mechanisms of High-Energy Nuclear Reactions
Phys Rev C8,2412 (1973) R.Madey, F.M.Waterman

Phys Rev C8,2419 (1973) F.M.Wateman, R.Madey

Astrophys Space

Sci 24,95(1973)

G.Carpenter,

C.S.Dyer

Symp Applications of Nucl Data in Sci \& Tech Paris,France Mar 121973

E.Ross, K.Baechmann

Studsvik report (Sweden) AE-479 (1973) L.Jeki

Dubna report JINR-P1-7352(1973) V.I.Komarov et al

At Energ(USSR) 35, 163(1973)

V.S.Barashenkov

V.D.Toneev

Izv Akad Nauk Azerb Ser Fiz Tekh 2,11 (1972)O.B.Abdinov

Dubna report JINR-E2-7278(1973) V.S.Barashenkov et al

Radiochim Acta 18, 133(1972)

K.Baechmann et al intranuclear cascade, neutron spectra

intranuclear cascade, neutron spectra

radiation effects

spallation

charge-exchange reactions

knock-out reactions

Monte Carlo method

cascade evaporation model, nuclear cascades

nuclear cascades

compound nucleus, knock-out reactions 
Cs

$27 \mathrm{Al}$
$12 \mathrm{C}$
$\mathrm{Si}$

Si

$\mathrm{Au}, \mathrm{Pb}$

$\mathrm{Bi}, \mathrm{U}$

$\mathrm{Al}, \mathrm{C}, \mathrm{Cu}$

$238 \mathrm{U}$

$209 \mathrm{Bi}$

$12 \mathrm{C}, 27 \mathrm{Al}$

$197 \mathrm{Au}$

$27 \mathrm{Al}, 9 \mathrm{Be}$

$2 \cap 9 \mathrm{Bi}, 12 \mathrm{C}$
Differential Recoil and Angu-

lar Distribution Study of

Several $(p, p x n)$ and $(p, x n)$

Reactions of 133 Cs with 550-

$\mathrm{MeV}$ Protons

Comparison of the Results of

Calculations Done at Different

Laboratories with Each Other

and with Experiments on the

Secondary Proton and Neutron

Spectra from Protons at 140

and $160 \mathrm{MeV}$ on Nuclei

Gamma Radiation Produced by the Inelastic Interaction of 600 -

MeV Protons with Carbon, Oxygen, Aluminum, and Silicon Nuclei

Recombination Centres in $\mathrm{N}$ - and P-Type Silicon Irradiated by

Very-High-Energy Protons

Study of Nuclear Fission Induced by High-Energy Protons

Low Energy Protons from C, Al, $\mathrm{Ni}, \mathrm{Cu}$, and $\mathrm{Au}$ Under $600 \mathrm{MeV}$ Proton Bombardment

Transport of Neutrons Induced by $800-\mathrm{MeV}$ Protons

Cascade Theory of Particle and Nuclei Interactions with Nuclei

Theoretical and Experimental Study of the Production of Light Fast Nuclei in High Energy Proton-Nucleus Interactions

Neutron and Proton Spectra from Targets Bombarded by $450-\mathrm{MeV}$ Protons
Carnegie-Melion U

Thesis(1973)

M.A.Molecke

NASA report

NASA-CR-130347

(1966) H.W.Bertini

Atomizdat(1972)

R.Ya.Zul'karneev

et al

Int Conf on Defects

in Semiconductors

(1972) Reading,UK

D.Bielle-Daspet

Rev Phys Appl 7,243

(1972) R.Brandt

et al

Thesis(1972)

Univ Microfilms

Order \#73-0́10

H.D.Orr III

Los Alamos report

I_A-UR-73-2C0(1972)

R.G.Fluharty et al

Dubna report

JINR-D1-6349

p.153(1971)

V.S.Barashenkov et al

J Phys (Paris) 33,

477(1972)

J.P.Alard et al

Phys Rev C6, 1496

(1972) J.W.Wachter

et al direct reactions,

Monte Carlo method

knock-out

reactions,

neutron spectra

gamma spectra

physical

radiation

fission

energy spectra.

spallation

neutron-beam

production

nuclear cascades

spallation

neutron spectra 
Al, C.Cu

$\mathrm{Au}, \mathrm{Pb}$

$238 \mathrm{U}$

$27 \mathrm{Al}, 64 \mathrm{Cu}$

$208 \mathrm{~Pb}, 7 \mathrm{Li}$

$12 \bar{c}$

$232 \mathrm{Th}$

$235,238 \mathrm{U}$

U

$27 \mathrm{Al}, 40 \mathrm{Ca}$

$12 \mathrm{C}$

$27 \mathrm{Al}$

$181 \mathrm{Ta}$
Low-Energy Proton Production

Under $600-\mathrm{MeV}$ Proton Bombard-

ment

Fluxes of Neutrons Generated

By High-Energy Protons in the

Thick Blocks of Uranium

Study of the Spectra of Fast

Deuterons Produced in the

Interaction of $670 \mathrm{MeV}$ Protons

with Nuclei

Total p- 12C Cross Section

Between 190 and $560 \mathrm{MeV}$

Calculations of Spallation-

Fission Competition in the

Reactions of Protons with

Heavy Elements at Energies

Less Than or Equal to $3 \mathrm{GeV}$

Optical Model Analysis of

Elastic Proton Scattering at

$180 \mathrm{MeV}$

Spectrum of Neutrons Produced

by $800-\mathrm{MeV}$ Protons on Uranium

To the Prucess of Deuteron

Knocking-Out from Nuclei by

High-Energy Protons

Mechanism of Deuteron Production in Inieraction of $670-\mathrm{MeV}$

Protons with Nuclei

Medium Energy Intranuclear Cascade Calculations: A Comparative Study

Synchrocyclotron Shield-Leakage Neutron Spectra
Trans Amer Nucl Soc

15,972(1972)

H.D.Orr et al

Dubna report JINR-

P16-6623(1972)

V.S.Barashenkov,

V.D.Toneev

Nucl Phys A195,581

(1972) L.S.Azhgirei

et al

FRNC-TH-238(1971)

Grenoble Univ

Thesis, B.Favier

Phys Rev C6,66

(1972) R.L.Hahn,

H.W.Bertini

Phys Scr 4,235

(1971)

A.Ingemarsson,

G.Tibell

Trans Amer Nucl

Soc 15,555(1972)

L.R.Veeser et al

Dubna report

JINR-P4-6299(1972)

B.N.Kalinkin,

VL.Shmonin

Dubna report

JINR-P2-6325(1972)

O.D.Dal'Karov,Q.Z.Ngo

Nucl Phys A187,531

(1972)

V.S.Barashenkov

et al

CERN-71-16 V1 \& 2

(1971) CERN report

VE.Aleinikov et al low energy

proton emission

Monte Carlo

calculations

deuteron spectra

total cross

sections

nuclear cascades

optical model

Monte Carlo

method,

neutron spectrum

quuasielastic

scattering,

deuteron knockout

particle

production

Monte Carlo

method,

nuclear cascades

flux leakage, radiation escape 


\begin{tabular}{|c|c|c|c|}
\hline $181 \mathrm{Ta}$ & $\begin{array}{l}\text { Interaction of High-Energy } \\
\text { Protons with Tantalum Nuclei. } \\
\text { Kinetic Energies and Excitation } \\
\text { Energies }\end{array}$ & $\begin{array}{l}\text { Radiochim Acta 16, } \\
129(1971) \\
\text { U.Trabitzsch, } \\
\text { K.Baechmann }\end{array}$ & $\begin{array}{l}\text { recoils, } \\
\text { spallation }\end{array}$ \\
\hline $\begin{array}{l}27 \mathrm{Al} \\
{ }^{9} \mathrm{Be}, \mathrm{B} \\
\mathrm{C}, \mathrm{Cu}\end{array}$ & $\begin{array}{l}\text { Reaction Cross Sections for } \\
\text { Protons in the Energy Range } \\
220 \text { to } 570 \mathrm{MeV}\end{array}$ & $\begin{array}{l}\text { Nucl Phys A183,81 } \\
\text { (1972) P.U.Renberg } \\
\text { et al }\end{array}$ & \\
\hline $\begin{array}{l}181 \mathrm{Ta} \\
238 \mathrm{U}\end{array}$ & $\begin{array}{l}\text { Neutron Production by Medium- } \\
\text { Energy Protons on Heavy Metal } \\
\text { Targets }\end{array}$ & $\begin{array}{l}\text { Los Alamos report } \\
\text { LA-4789(1972) } \\
\text { R.R.Fullwood et al }\end{array}$ & $\begin{array}{l}\text { particle } \\
\text { production, }\end{array}$ \\
\hline \multirow[t]{2}{*}{$\mathrm{Pb}$} & $\begin{array}{l}\text { Neutron Yield from Protons and } \\
\text { Deuterons of Momenta Between } \\
0.85 \text { and } 1.7 \mathrm{GeV} / \mathrm{C} \text { Totally } \\
\text { Absorbed in Lead }\end{array}$ & $\begin{array}{l}\text { Can J Phys } 49,2061 \\
\text { (1971) D.Wesh } \\
\text { E.Wood }\end{array}$ & neutron yield \\
\hline & $\begin{array}{l}\text { Cross Sections for Summed } \\
\text { Quasi-Elastic and Inelastic } \\
\text { Scattering }\end{array}$ & $\begin{array}{l}\text { Nucl Phys A173,225 } \\
\text { (1971) D.F Jackson }\end{array}$ & $\begin{array}{l}\text { direct reactions, } \\
\text { quasi-elastic } \\
\text { scattering }\end{array}$ \\
\hline $\begin{array}{l}27 \mathrm{Al} \\
\mathrm{Cu}, \mathrm{Fe}\end{array}$ & $\begin{array}{l}\text { Measurements of Spallation } \\
\text { Cross Sections for } 590 \mathrm{MeV} \\
\text { Protons on Thin Targets of } \\
\text { Copper, Nickel, Iron, and } \\
\text { Aluminum }\end{array}$ & $\begin{array}{l}\text { Nucl Phys A169,437 } \\
\text { (1971) J.E.Cline, } \\
\text { E.B.Nieschmidt }\end{array}$ & spallation \\
\hline $\begin{array}{l}27 \mathrm{Al} \\
65 \mathrm{Cu}\end{array}$ & $\begin{array}{l}\text { Results from Medium-Energy } \\
\text { Intranuclear-Cascade } \\
\text { Calculations }\end{array}$ & $\begin{array}{l}\text { Nucl Phys A169,670 } \\
\text { (1971) H.W.Bertini, } \\
\text { M.P.Guthrie }\end{array}$ & $\begin{array}{l}\text { intranuclear } \\
\text { cascade }\end{array}$ \\
\hline $\begin{array}{l}27 \mathrm{Al}, \mathrm{Co} \\
\mathrm{C}, \mathrm{Na}\end{array}$ & $\begin{array}{l}\text { Interaction of High-Energy } \\
\text { Radiation with Matter }\end{array}$ & $\begin{array}{l}\text { Dubna report } \\
\text { JINR-P2-5719(1971) } \\
\text { V.S.Barashenkov et al }\end{array}$ & Monte Carlo method \\
\hline $\mathrm{C}$ & $\begin{array}{l}\text { Production of Fast Deuterons } \\
\text { from the Interaction of High- } \\
\text { Energy Protons with Nuclei }\end{array}$ & $\begin{array}{l}\text { Nucl Phys A163,465 } \\
\text { (1971) } \\
\text { V.V.Balashov, V.I.Markov }\end{array}$ & $\begin{array}{l}\text { impulse } \\
\text { approximation }\end{array}$ \\
\hline \multirow[t]{2}{*}{$12 \mathrm{C}$} & $\begin{array}{l}\text { Spectrum of Fast Deutercns } \\
\text { Emitted in } 670 \text {-MeV Proton } \\
\text { Interaction with Carbon Nuclei }\end{array}$ & $\begin{array}{l}\text { Yadern Fiz 13,6 } \\
\text { (1971) } \\
\text { L.S.Azhgirei et al }\end{array}$ & spectra \\
\hline & $\begin{array}{l}\text { Attenuation of a High-Energy } \\
\text { Neutron Flux in a Cylindrical } \\
\text { Duct }\end{array}$ & $\begin{array}{l}\text { At Energ(USSR) 29, } \\
378(1970) \\
\text { N.I.Bushuev et al }\end{array}$ & $\begin{array}{l}\text { neutron flux, } \\
\text { shielding }\end{array}$ \\
\hline $238 \mathrm{U}$ & $\begin{array}{l}\text { Precompound Decay from a Time- } \\
\text { Dependent Point of View }\end{array}$ & $\begin{array}{l}\text { Columbia Univ report } \\
\text { CU-1019-79(1970) } \\
\text { G.D.Harp, J.M.Miller }\end{array}$ & neutron spectra \\
\hline
\end{tabular}


Table I (Cont.)

${ }^{209} \mathrm{Bi}$

$238 \mathrm{U}$

$27 \mathrm{Al}$

$58 \mathrm{Ni}$

$197 \mathrm{Au}$

$\mathrm{Ge}$

$\mathrm{Si}, \mathrm{Ge}$

${ }^{9} \mathrm{Be},{ }^{209} \mathrm{Bi}$

$\mathrm{Ca}, \mathrm{C}, \mathrm{Cu}$

$\mathrm{U}$

$63 \mathrm{Cu}$

$56 \mathrm{Fe}$

${ }^{9} \mathrm{Be}, 12 \mathrm{C}$

$\mathrm{Fe}$

$2 \mathrm{CO}_{\mathrm{Bi}}$

$238 \mathrm{U}$
Measurement of the Prompt Neu-

trons Emitted in the Fission of

$209 \mathrm{Bi}$ and $238 \mathrm{U}$ Induced by $155-$

MeV Protons

Angular Dependences of Many-

Charged Particles Produced by

$660-\mathrm{MeV}$ Protons on $27 \mathrm{Al}$ Nuclei

Angular Distribution of Recoil

Products from Some Simple

Reactions of $400 \mathrm{MeV}$ Protons

with $58 \mathrm{Ni}$ and $197 \mathrm{Au}$

Oscillations of the Photo-

current in $\mathrm{Ge}$ with Radiation

Defects

Effects of Very-High-Energy

Protons on Electric Properties

of Silicon and Germanium

Intranuclear-Cascade Calcula-

tion of the Secondary Nucleon

Spectra from Nucleon-Nucleus

Interactions in the Energy

Range 340 to $2900 \mathrm{MeV}$ and

Comparisons with Experiment

A Recoil Study of (p,n) and

$(p, 2 n)$ Reactions for Proton

Bombarding Energies of 100 to $400 \mathrm{MeV}$

Neutron Pickup Reactions in $9 \mathrm{Be}, 12 \mathrm{C}$, and $28 \mathrm{Si}$ Induced by $185 \mathrm{MeV}$ Protons

Interaction Cross Sections of High Energy Particles with

Atomic Nuclei

Calculation of the ResidualPhoton Dose Rate Induced in Iron by $200-\mathrm{MeV}$ Protons

Comparative Study of the Fission of Bismuth and Uranium Induced by High-Energy Protons $(156 \mathrm{MeV})$
Phys Rev C2,256

(1970) E.Cheifetz

et al

Yadern Fiz 12,234

(1970)

Y.P.Milovanov et al

Nucl Phys A152,295

(1970) Y.Yu et al

angular

distribution

of recoils

Sov Phys Semicond

3, 1294(1970)

E.E.Rubinova et al

NP-18474(1970)

Toulouse Univ

(France) Thesis

D.Bielle-Daspet

Phys Rev 188,1711

(1969) H.W.Bertini

No Information

Thesis(1969)

A.C.Stalker

Ark Fys $\underline{39}, 323$

pick-up reactions

(1969) O.Sundberg,

J.Kaellne

Oak Ridge report

electric

properties, radiation effects

intranuclear-

cascade

calculations

evaporation

model

Glauber theory

ORNL-TR-2235(ND)

S.M.Eliseev

Nucl Sci Eng $\underline{38}$, 271(1969)

gamma radiation

T.W.Armstrong, J.Barish

Nucl Phys A134,513

(1969) J.Galin

et al liquid drop model fission 
Table I (Cont.)

$\begin{array}{ll}\mathrm{Pb}, \mathrm{Sn} & \begin{array}{l}\text { Ranges and Ionization Energy } \\ \mathrm{Cu}, \mathrm{Al}\end{array} \\ & \begin{array}{l}\text { Sosses of Protons in Various } \\ \text { Substances }\end{array} \\ { }^{27} \mathrm{Al}, \mathrm{C} & \begin{array}{l}\text { Calculation of the Neutron and } \\ \text { Proton Spectra from Thick } \\ \text { Targets Bombarded by } 450-\mathrm{MeV} \\ \text { Protons and Comparison with } \\ \text { Experiment }\end{array} \\ & \text { Experimental Study of the } \\ & \text { Knockout of Fast Light Nuclei } \\ & \text { in } 600 \mathrm{MeV} \text { Proton Interactions } \\ & \text { with Nuclei }\end{array}$

$27 \mathrm{Al}, 6 \mathrm{Li}$

$7_{\mathrm{Li}, \mathrm{Cu}, \mathrm{Pb}}$

$A>40$

Sb,U
12C Yield of Deuterons Knocked Out from the $12 \mathrm{C}$ Nucleus by 730 and $1260 \mathrm{MeV}$ Protons
$\mathrm{Ge} \quad$ Studies of Radiation Defects in Germanium Irradiated by High-Energy Protons

$\begin{array}{ll}24 \mathrm{Mg} & \text { Inelastic Scattering of } 100 \\ 28 \mathrm{Si} & \begin{array}{l}\mathrm{MeV} \text { Protons from } \mathrm{Mg} \text { and } \mathrm{Si} \\ \text { Using a Ge(Li) Total Absorp- } \\ \text { tion Proton Counter }\end{array} \\ & \begin{array}{l}\text { Quasielastic Scattering of } \\ 12 \mathrm{C}, 9 \mathrm{Be}\end{array} \\ 665-\mathrm{MeV} \text { Protons by Alpha- } \\ 6 \mathrm{Li} & \begin{array}{l}\text { Particle Clusters in Light } \\ \text { Nuclei }\end{array}\end{array}$

Yadern Fiz 9,997

(1969)

I.M.Vasilevskii

et al

Nucl Sci Eng 36,291

(1969)

R.G.Alsmiller

et al

Study Session on

Recent Developments

in Theoretical

Nuclear Physics,

LaToussuire,France

Lycen-7302,p.54.1

(1973) J.P.Alard et al

Dubna report

JINR-P1-6308(1972)

L.S.Azhgirei et al

Univ of Minnesota

report COO-1265-59

(19ND) F.D.Becchet-

ti Jr.,G.W.Greenlees

$J$ Inorg Nucl Chem

31,2649(1969)

E.Hageboe, H.Ravn

JETP Lett (USSR) 9, 413(1969)

V.S.Borisov et al

Fiz Tekh Poluprov

3,1119(1969)

R.F.Konopleva

et al

Nucl Phys A134,577

(1969) Y.S.Horowitz

et al

Brookhaven Lab

report BNL-TR-282

(19ND) V.I.Komarov ett al ionization

neutron and

proton yields

knockout reaction thermodynamic model

spectra

elastic scattering, optical model,

recoils

deuteron yields

electric properties, radiation effects, semiconductors

inelastic scattering

quasielastic scattering 
${ }^{9} \mathrm{Be}$

$12 \mathrm{C}$

$6 \mathrm{Li}$

$\operatorname{Re}$

$\mathrm{Pb}$

$27 \mathrm{Al}, 40 \mathrm{Ca}$

$12 \mathrm{C}, 208 \mathrm{~Pb}$

$58 \mathrm{Ni}$

$27 \mathrm{Al}, \mathrm{Pb}$

$\mathrm{C}, \mathrm{Fe}, \mathrm{Cu}$

$\mathrm{U}, \mathrm{Ti}$

Al,Cd

$\mathrm{C}, \mathrm{Cu}$

$\mathrm{Al}, \mathrm{C}, \mathrm{Au}$

$\mathrm{Li}, \mathrm{Mg}$

U
Knock-Out of Fast 3 He Fragments from Light Nuclei by

665-MeV Protons

Inelastic Interactions of

High-Energy Protons with

Atomic Nuclei

Thermal Annealing of Crystal-

line Rhenium Compounds Irradi-

ated with $660 \mathrm{MeV}$ Protons

Nuclear Charge Dispersion in

the Fission of $235 \mathrm{U}$ and $238 \mathrm{U}$

with 20 to $85 \mathrm{MeV}$ Protons

The Transport of Neutrons

Produced by 3-GeV Procon-Lead

Nucleus Collisions Through a

Labyrinth and Comparison with

Experiment

Elastic Scattering of 61.4

MeV Protons

Energy Dependence of Interaction Cross Sections of Nucleons with Atomic Nuclei at Energies Higher Than 50 $\mathrm{MeV}$

Gamma Radiation from $\mathrm{Li}, \mathrm{C}$, $\mathrm{Al}, \mathrm{Ni}, \mathrm{Cu}, \mathrm{Nb}, \mathrm{Cd}, \mathrm{Ta}$, and $\mathrm{Pb}$ Targets Bombarded by 21 MeV Protons

Gamma Radiation from $\mathrm{Li}, \mathrm{C}$, $\mathrm{Mg}, \mathrm{Al}, \mathrm{Ni}, \mathrm{Zn}, \mathrm{Ag}, \mathrm{Ta}, \mathrm{Au}$, and $\mathrm{Pb}$ Targets Bombarded by 42-MeV Alpha Particles

Yields and Recoil Properties of Scandium Isotopes Produced in the Interaction of $590 \mathrm{MeV}$ and $18 \mathrm{GeV}$ Protons with Uranium
Brookhaven Lab

report BNL-TR-281

(19ND) V.I.Komarov

Oak Ridge report

ORNL-TR-2200(19ND)

V.S.Barashenkov

et al

$\mathrm{J}$ Inorg Nucl Chem

31, 2669(1969)

E.Ianovici, N.Zaitseva

Thesis(1968) no

university infor-

mation given, A.H.Khan

Nucl Instr Meth 73 ,

280(1969)

R.G.Alsmiller,

E.Solomito

Phys Rev 181,1565

(1969) O.Karban

Oak Ridge report

ORNL-TR-2148(19ND)

V.S.Barashenkov et al

At Energ (USSR) 26, 388(1969) S.S.Omarov

et al

At Energ (USSR) 26 .

gamma radiation 390(1969) S.S.Omarov et a!

$\mathrm{J}$ Inorg Nucl Chem 31,1883(1966)

H.Ravn elastic

scattering,

optical model

inelastic

scattering, Monte Carlo method, intranuclear cascades

radiation

effects, recoils

fission, fission

products

neutrons/transport

gamma dose rates recoil properties 
$\mathrm{Te}$

$27 \mathrm{Al}$

Al,Si

0

$\mathrm{Pb}, \mathrm{Al}$

$\mathrm{C}, \mathrm{Cu}$

$40 \mathrm{Ca}, 12 \mathrm{C}$

$58 \mathrm{Ni}, 120 \mathrm{Sn}$

$208 \mathrm{~Pb}$

$52 \mathrm{Cr}, 63 \mathrm{Cu}$

$77 \mathrm{Se}, 124 \mathrm{Te}$

$27_{\mathrm{Al}, \mathrm{C}}$

$\mathrm{Cu}$

Cs

$12 \mathrm{C}$
Simple Nuclear Reactions on the Separated Isotopes of Tellurium at $300 \mathrm{MeV}$

Angular Distribution of $24 \mathrm{Na}$ Recoil Nuclei Produced in the Reaction $27 \mathrm{Al}(\mathrm{p}, 3 \mathrm{pn})$

Investigation of Spallation of the Nuclei F, Mg, Al, Si with Fragments, Induced by High-Energy Protons

Study of $660 \mathrm{MeV}$ Proton Interaction with Oxygen Nuclei

Ranges and lonization Energy Losses of Protons in Various Substances

Optical Potential in ProtonNucleus Scattering

Elastic and Inelastic Scattering of 155-MeV Protons on $208 \mathrm{~Pb}$

The Measurement of Some $(p, n)$ and $(p, 2 n)$ Reaction Cross Sections at $400 \mathrm{MeV}$

Calculation of the Neutron and Proton Spectra from Thick Targets Bombarded by $450-\mathrm{MeV}$ Protons and Comparison with Experiment

The Behaviour of Radio-lodine Recoils Formed by Neutron and Proton Irradiation of Some Solid Cesium Compounds

Recoil Study of the $12 \mathrm{C}(\mathrm{p}, \mathrm{pn})$ Reaction from Threshold to $150 \mathrm{MeV}$
Thesis(1968) no university information given R.F.Schall Jr

Yadern Fiz 9,930 (1969) F.P.Denisov et al

Yadem Fiz 9,1155 (1969) G.A.Fedoseev et al

Yadern Fiz 9,925 (1969) V.E.Dudkin et al

Yadern Fiz 9,997 (1969) I.M.Vasilevskii et al

Michigan State Univ report COO-105133(1969) D.A.Slanina

J Phys (Paris) 30 , 13(1969) A.Willis et al

J Inorg Nucl Chem 31,1915(1969)

D.J.Reuland,

A.A.Caretto Jr

Nucl Sci Eng 36, 291 (1969) R.G.Alsmiller Jr., et al

I Inorg Nucl Chem 31,2311(1969)

I.Dema, N.G.Zaitseva

Nucl Phys A130,88 (1969) N.T.Porile $(p, 2 p),(p, p n)$ and $(\mathrm{n}, 2 \mathrm{n})$

(p,3pn), angular distribution of recoils

spallation

charged-particle spectra

proton range

optical model

elastic and inelastic scattering yields

$(p, n)$ and $(p, 2 n)$

reactions in thick targets

het.ting, recoils

recoil ranges, cascade calculation 


\begin{tabular}{|c|c|c|c|}
\hline${ }^{9} \mathrm{Be}$ & $\begin{array}{l}\text { Quasi-Free Scattering in the } \\
\text { Reaction } 9 \mathrm{Be}(\mathrm{p}, \mathrm{p} \text { alpha) } 5 \mathrm{He} \\
\text { at } 55 \mathrm{MeV}\end{array}$ & $\begin{array}{l}\text { J Phys Soc Japan 26, } \\
\text { 1078(1969) } \\
\text { S.Yamashita et al }\end{array}$ & $\begin{array}{l}\text { quasi-free } \\
\text { scattering }\end{array}$ \\
\hline $\begin{array}{l}27 \mathrm{Al}, 12 \mathrm{C} \\
59 \mathrm{Co}\end{array}$ & $\begin{array}{l}\text { Total Reaction Cross Section } \\
\text { Measurements with } 60-M \text { M }^{\prime} \\
\text { Protons }\end{array}$ & $\begin{array}{l}\text { Phys Rev Lett 22, } \\
1128(1969) \\
\text { J.J.Menet et al }\end{array}$ & $\begin{array}{l}\text { total cross } \\
\text { sections }\end{array}$ \\
\hline Dy & $\begin{array}{l}\text { Note on the L- and M-Shell } \\
\text { Ionization by Heavy Charged } \\
\text { Particles }\end{array}$ & $\begin{array}{l}\text { Phys Lett A29, } 281 \\
\text { (1969) J.M.Hans- } \\
\text { teen, O.P.Moss bekk }\end{array}$ & $\begin{array}{l}\text { perturbation } \\
\text { theory, L- and M- } \\
\text { shell ionization }\end{array}$ \\
\hline \multirow[t]{2}{*}{$\begin{array}{l}\mathrm{Zr}, \mathrm{Te} \\
\mathrm{Hg}, \mathrm{Ge}\end{array}$} & $\begin{array}{l}\text { Study of }(p, x n) \text { Reactions } \\
\text { at } 400 \mathrm{MeV}\end{array}$ & $\begin{array}{l}\text { Phys Rev } 178,1732 \\
\text { (1969) L.B.Church, } \\
\text { A.A.Caretto Jr }\end{array}$ & $\begin{array}{l}\text { excitation } \\
\text { functions }\end{array}$ \\
\hline & $\begin{array}{l}\text { Synchrccyclotron Proton Beam } \\
\text { with an Energy of } 100 \text { to } 200 \\
\text { MeV for Medico-Biological } \\
\text { Investigations }\end{array}$ & $\begin{array}{l}\text { Med Radiol } 14,54 \\
\text { (1969) V.P.Dzhele- } \\
\text { pov et al }\end{array}$ & radiation sources \\
\hline O & $\begin{array}{l}\text { Formation Cross Sections of } \\
\text { Lithium, Beryllium, and Boron } \\
\text { Isotopes Produced by the } \\
\text { Spallation of Oxygen by High- } \\
\text { Energy Protons }\end{array}$ & $\begin{array}{l}\text { J Geophys Res 74, } \\
2447(1969) \text { F.Yiou } \\
\text { et al }\end{array}$ & spallation \\
\hline $\mathrm{Pb}$ & $\begin{array}{l}\text { Neutron Yields and Thermal } \\
\text { Neutron Fluxes in System } \\
\text { Lead-Water, Bombarded by } \\
\text { High Energy Protons }\end{array}$ & $\begin{array}{l}\text { At Energ (USSR) } 25 \text {, } \\
479(1968) \\
\text { R.G.Vasil'kov et al }\end{array}$ & neutron yields \\
\hline $\begin{array}{l}\mathrm{C}, \mathrm{Cu} \\
\mathrm{Al}\end{array}$ & $\begin{array}{l}\text { Range and Ionization Losses } \\
\text { of Proton Energy in Different } \\
\text { Materials }\end{array}$ & $\begin{array}{l}\text { Argonne Lab report } \\
\text { ANL-TRANS-724(19ND) } \\
\text { I.M.Vasilevskii et al }\end{array}$ & $\begin{array}{l}\text { ionization energy } \\
\text { losses }\end{array}$ \\
\hline $\begin{array}{l}59 \mathrm{Co} \\
63,65 \mathrm{Cu}\end{array}$ & $\begin{array}{l}\text { Evaporation Step in Spalla- } \\
\text { tion Reactions }\end{array}$ & $\begin{array}{l}\text { Nucl Phys A.125,401 } \\
\text { (1969) G.Rudstam }\end{array}$ & $\begin{array}{l}\text { evaporation } \\
\text { theory, spailation }\end{array}$ \\
\hline $\begin{array}{l}\mathrm{U}, \mathrm{Al},{ }^{9} \mathrm{Be} \\
\mathrm{Cd}, \mathrm{C}, \mathrm{Cu} \\
\mathrm{Fe}, \mathrm{Pb}, \mathrm{Ti}\end{array}$ & $\begin{array}{l}\text { Energy Dependence of the } \\
\text { Nuclear Cross Sections for } \\
\text { Nucleons at Energy Above } 50 \\
\mathrm{MeV}\end{array}$ & $\begin{array}{l}\text { Dubna report JWNR- } \\
\text { P2-4183(1968) } \\
\text { V.S.Barashenkov et al }\end{array}$ & $\begin{array}{l}\text { cross sections, } \\
\text { energy dependence }\end{array}$ \\
\hline $12 \mathrm{C}$ & $\begin{array}{l}\text { Scattering of } 100 \mathrm{MeV} \text { Protons } \\
\text { by } 12 \mathrm{C}\end{array}$ & $\begin{array}{l}\text { Nucl Phys A124,662 } \\
\text { (1969) R.M.Haybron }\end{array}$ & $\begin{array}{l}\text { impulse } \\
\text { approximation, } \\
\text { elastic scattering }\end{array}$ \\
\hline
\end{tabular}


Table I (Cont.)

\begin{tabular}{|c|c|c|c|}
\hline \multirow[t]{2}{*}{$\mathrm{C}, \mathrm{Cu}$} & $\begin{array}{l}\text { Measuremit and Characteris- } \\
\text { tics of Depin-Dose Patterns } \\
\text { Due to Proton Beams }\end{array}$ & $\begin{array}{l}\text { Health Phys } 16,349 \\
\text { (1969) N.A.Baily, } \\
\text { H.S.Frey }\end{array}$ & energy losses \\
\hline & $\begin{array}{l}\text { Study of the Correlation of } \\
\text { the Characteristics of Nuc- } \\
\text { lear Spallation Products with } \\
\text { Their Fragments }\end{array}$ & $\begin{array}{l}\text { Izv Akad Nauk Uzb } \\
\text { SSR Ser Fiz-Mat } 3 \text {, } \\
\text { 36(1968) S.A.Azimov } \\
\text { et al }\end{array}$ & $\begin{array}{l}\text { recoils, } \\
\text { spallation } \\
\text { products }\end{array}$ \\
\hline $232 \mathrm{Th}$ & $\begin{array}{l}\text { Nuclear Charge Dispersion in } \\
\text { the Fission of } 232 \mathrm{Th} \text { by } \\
\text { Protons of Energy } 20 \text { to } 85 \\
\mathrm{MeV}\end{array}$ & $\begin{array}{l}\text { Can J Chem 47,301 } \\
\text { (1969) P.P.Benjamin } \\
\text { et al }\end{array}$ & $\begin{array}{l}\text { fission } \\
\text { products }\end{array}$ \\
\hline \multirow[t]{3}{*}{$\begin{array}{l}{ }^{209} \mathrm{Bi} \\
54 \mathrm{Fe}, 12 \mathrm{C}\end{array}$} & $\begin{array}{l}\text { Differential Cross Sections } \\
\text { for thi Charged Particles } \\
\text { Produced by } 60-\mathrm{MeV} \text { Protons } \\
\text { on Carbon, Iron, and Bismuth }\end{array}$ & $\begin{array}{l}\text { Thesis(1968) no } \\
\text { university infor- } \\
\text { mation given } \\
\text { F.E.Bertrand Jr }\end{array}$ & $\begin{array}{l}\text { inelastic } \\
\text { scattering }\end{array}$ \\
\hline & $\begin{array}{l}\text { Shielding Calculations for a } \\
\text { 200-MeV Proton Accelerator }\end{array}$ & $\begin{array}{l}\text { Oak Ridge report } \\
\text { ORNL-4336(1968) } \\
\text { R.G.Alsmiller Jr et al }\end{array}$ & $\begin{array}{l}\text { neutron transport } \\
\text { calculations }\end{array}$ \\
\hline & $\begin{array}{l}\text { Cross Sections for Fast Par- } \\
\text { ticles and the Atomic Nuclei }\end{array}$ & $\begin{array}{l}\text { Dubna report } \\
\text { JDNR-P2-4068(1968) } \\
\text { V.S.Barashenkov et al }\end{array}$ & data compilation \\
\hline $238 \mathrm{U}$ & $\begin{array}{l}\text { Yields of Some Light-Mass } \\
\text { Nuc!ides Produced in the } \\
\text { Proton-Induced Fission of } \\
238 \mathrm{U}\end{array}$ & $\begin{array}{l}\text { Can J Chem } 46,3565 \\
\text { (1968) A.H.Khan } \\
\text { et al }\end{array}$ & $\begin{array}{l}\text { fission at } 20 \\
\text { to } 85 \mathrm{MeV}\end{array}$ \\
\hline $\begin{array}{l}65 \mathrm{Cu} \\
197 \mathrm{Au}\end{array}$ & $\begin{array}{l}\text { Angular Distributions of } \\
\text { Recoil Products from Some } \\
\text { Reactions Indiced in } 65 \mathrm{Cu} \\
\text { and } 197 \mathrm{Au} \text { by Protons of } \\
\text { Energy } 30 \text { to } 85 \mathrm{MeV}\end{array}$ & $\begin{array}{l}\text { Can J Chem 46, } 3551 \\
\text { (1968) M.K.Dewanjee } \\
\text { et al }\end{array}$ & $\begin{array}{l}\text { recoils. }(p, p n) \\
\text { and }(p, p 3 n)\end{array}$ \\
\hline $\begin{array}{l}\mathrm{Pb}, \mathrm{U} \\
\mathrm{Be}\end{array}$ & $\begin{array}{l}\text { Thermal-Neutron Flux Cener- } \\
\text { ation by High-Energy Protons }\end{array}$ & $\begin{array}{l}\text { Nucl Sci Eng } \underline{34,104} \\
\text { (1968) W.A.Coleman, } \\
\text { R.G.Alsmiller Jr }\end{array}$ & thermal neutrons \\
\hline $127 I$ & $\begin{array}{l}(p, n) \text { and }(p, p \times n) \text { Reactions } \\
\text { in } 127 I\end{array}$ & $\begin{array}{l}\text { Can J Chem } 46,3171 \\
\text { (1968) V.P.Narang, } \\
\text { L. Yaffe }\end{array}$ & $\begin{array}{l}(p, n) \text { and }(p, p \times n) \\
\text { up to } 80 \mathrm{MeV}\end{array}$ \\
\hline $\begin{array}{l}65 \mathrm{Cu} \\
197 \mathrm{Au}\end{array}$ & $\begin{array}{l}\text { Average Recoil Ranges of }{ }^{64} \mathrm{Cu} \\
\text { and } 196 \mathrm{Au} \text { Produced by Protons } \\
\text { of } 20 \text { to } 85-\mathrm{MeV} \text { Energy in }\end{array}$ & $\begin{array}{l}\text { Can J Chem 46,3157 } \\
\text { (1968) M.K.Dewanjee } \\
\text { et al }\end{array}$ & $\begin{array}{l}(p, p n), \text { average } \\
\text { recoil ranges }\end{array}$ \\
\hline
\end{tabular}


Si

O,Ar

$\mathrm{He}, \mathrm{N}$

$\operatorname{Sr}, Y$

concrete

${ }^{7 \mathrm{Li}, 9 \mathrm{Be}}$

$11 \mathrm{~B}, \mathrm{C}, \mathrm{O}$

$\mathrm{Mg}, 27_{\mathrm{Al}}$

$\mathrm{Fe}$

$\mathrm{Ti}$

$27 \mathrm{Al}$

$12 i$

$65 \mathrm{Cu}$

$238 \mathrm{U}$

$\mathrm{CO}_{2}$

$11 \mathrm{~B}, \mathrm{~S}$
Excitation Functions for

Radioactive Isotopes Produced by Proton-Induced Reactions in Silicon

Calculations on Electron Capture from $\mathrm{D}, \mathrm{He}, \mathrm{N}, \mathrm{O}$, and $\mathrm{Ar}$ by Protons

Gamma Emission for $81 \mathrm{Rb},{ }^{82} \mathrm{Rb}$, $83 \mathrm{Rb}, 84 \mathrm{Rb}$ and $86 \mathrm{Rb}$

Back Yield of Neutrons from Shielding Bombarded with 660 MeV Protons

Gamna Rays from Bombardment of Light and Intermediate Weight Nuclei by 16- to 160 MeV Protons and 59-MeV Alpha Particles

Flux and Dose of Secondary Nucleons on Irradiating a Plane Titanium Shield with 660-MeV Protons

Comparison of Calculated Radiochemical Cross Sections with Experimental Results for Incident Protons and $\mathrm{Pi}^{-}$ Mesons in the 50- to $400-\mathrm{MeV}$ Region:Effect of Varying a Few Nuclear Parameters in the Calculations

Statistical Fluctuations of Energy Deposited in Low-Atomic Number Materials by $43.7-\mathrm{MeV}$ Protons

Measurement and Analysis of High-Energy Radiation through Activation Detectors Applications in Dosimetry
Phys Rev 172,1094 (1968) D.W.Sheiffey

reactions below $60 \mathrm{MeV}$

et al

J Phys B1,529(1968)

R.A.Mapleton

Izv Akad Nauk SSSR

Ser Fiz 31, 1661

(1967) Ya.Vrzal

et al

At Energ (USSR) 24 , 395(1968) L.R.Kimel et al

Nucl Sci Eng 32,392 (1968) W.Zobel et al

At Energ (USSR) 24 , 73(1968)

V.A.Kon'shin et al

Oak Ridge report ORNL-4 105(1968) H.W.Bertini reactions at 20 to $100 \mathrm{MeV}$, cross sections for electron capture

gamma spectra from proton reactions at $660 \mathrm{MeV}$

neutron yield

gamma spectra

secondary nucleons

(p,xpyn), cascadeevaporation model
Phys Rev 168,290 (1968) J.W.Hilbert et al

Saclay report CEA-R-3376(1968) L.Sklavenitis 
$12 \mathrm{C}$

Ag, In

In, Ag

$\mathrm{Li}, \mathrm{O}$

$\mathrm{Be}, \mathrm{C}$

$\mathrm{Si}$

160

$12 \mathrm{C}$

$\mathrm{Al}, \mathrm{Cu}$

$\mathrm{Fe}$

U
$\mathrm{Al}, \mathrm{Be}, \mathrm{Bi}$
$\mathrm{C}, \mathrm{Co}, \mathrm{Cu}$
$\mathrm{Cu}, \mathrm{Li}$
Neutron and Proton Spectra from Targets Bombarded by 160-MeV Protons
A Study by Means of Secondary Reactions of Fragments Produ- ced in Copper by High-Energy Protons and Alpha Particles

Recoil Studies of the Reaction

${ }^{12} \mathrm{C}$ (p.pn) ${ }^{11} \mathrm{C}$ from Threshold to $85 \mathrm{MeV}$

Recoil Properties of Products from the Spallation of Silver and Indium

Nuclear Reactions of Silver and Indium with 200- and 400MeV Protors

Formation of Helium Isotopes in Cosmic Rays

Excitation Functions of Radionuclides Produced by 0 to 60 $\mathrm{MeV}$ Proton Reactions in Silicon

Calculation of Spallation of $12 \mathrm{C}$ and 160 by $70-$ to $200-\mathrm{MeV}$ Protons

Neutron Shielding of the Los Alamos Meson Physics Facility

Excitation Functions for Radioactive Isotopes Produced by Procons Below $60 \mathrm{MeV}$ on $\mathrm{Al}, \mathrm{Fe}$, and $\mathrm{Cu}$

Yields and Isomeric Yield Ratios of Antimony Isotopes from the Interaction of 159$\mathrm{MeV}$ to 18.2-GeV Protons with Uranium
Can J Chem 46,267

(1968) S.Hontzeas

$(p, p n)$

Phys Rev 165,1281

(1968) J.A.Panontin

et al

spallation, recoil ranges

Phys Rev 165,1283 (1968) J.A.Panontin et al

Izv Akad Nauk SSSR Ser Fiz 30, 1778

reactions with 660 $\mathrm{MeV}$ protons

(1966) A.P.Zhadanov et al

excitation

Oak Ridge report

ORNL-TM-2060(1968)

D.W.Sheffey functions

J Phys (Paris) $\underline{28}$,

spallation

745(1967)

M.Epherre, E.Gradsztajn

New York Operations

Office(AEC) report

HASL-188(1967) K.O'Brien

neutron shielding

Phys Rev 162,1055

(1967)

I.R.Williams, C.B.Fulmer

excitation

functions

$\mathrm{J}$ Inorg Nucl Chem

excitation 29,2515(1967)

E.Hagebo

functions

Phys Rev 161,971 (1967) J.W.Wachter

et al

No university information given, Thesis (1966) K.E.Dierenfeldt 
$9 \mathrm{Be}$

12C

Ag, Ta, Au

$\mathrm{Pt}, \mathrm{Pb}$

$\mathrm{Al}, 65 \mathrm{Cu}$

$209 \mathrm{Bi}$

W

$\mathrm{KCl}$

$\mathrm{Ni}, \mathrm{C}$
Analysis of the Scattering of $46 \mathrm{MeV}$ Protons from ${ }^{9} \mathrm{Be}$ and $12 \mathrm{C}$

Effects of Various Doping Treatments Upon the Damage Production and Recovery of Deuteron-irradiated Aluminum

Deuterons, Tritons, and Helium Ions from Direct interaction Events of $156-\mathrm{MeV}$ Protons with Medium and Heavy Nuclei

Secondary Particle Spectra from the Interaction of 30 to $340 \mathrm{MeV}$ Protons on Complex Nuclei: Experimentai Data and Comparison wiü! Theory

Neutron Production in Ag, Ta, $\mathrm{Au}, \mathrm{Pt}$, and $\mathrm{Pb}$ by the interaction of 7.5 to $40 \mathrm{MeV}$ Protons

Energy Spectra from Inelastic Nuclear Reactions at Energies Below $450 \mathrm{MeV}$

Energy Spectra of Charged Particles Accompanying Fission of $W$ by Protons with $E_{p}=660$ $\mathrm{MeV}$

Relation of Radio- and Radiative-Chemical Effects in $\mathrm{KCl}$ Crystals Irradiated by High-Energy Protons

Proton-Nucleus Reaction Cross Sections Computed from Elastic Scattering Data

Energy and Spatial Distribution of a Stream of Secondary Veutrons Prodiced by $660-\mathrm{MeV}$ Protons in Blocks of Graphite and Nickel
Nucl Phys A100,497

(1967) G.R.Satchler

Phys Rev 158,661

(1967)

K.Herschbach,

J.J Jackson

J Fhys (Paris) 28 , 257(1967) H.Dubost et al

Oak Ridge report ORNL-P-2894(1974) H.W.Bertini

Phys Rev 159,1022 (1967) P.G.Thomas, W.Bartolini

American Nuclear Society, ANS-SD-3

Session 1, Paper 3 (19ND) H.W.Bertini

Yadem Fiz 5,727

(1967)

V.S.Bychenkov, N.A.Perfilov

Radiatsionnaya Fizika IV. Ionnye Kristally p. 13 (1966) R.V.Bogdanov, A.N.Murin

Can J Phys 45,1497 (1967) F.Sannes, D.G.Stairs

Atomizdat 2,226 (1966) V.A.Kon'shin et al inelastic

scattering

annealing, irradiation, radiation effects

emission, knockout reactions

emission spectra inelastic scattering

fission

irradiation, light

elastic scattering

angular distributions 


\begin{tabular}{|c|c|}
\hline $\begin{array}{l}\mathrm{Al}, \mathrm{C} \\
\mathrm{Fe}\end{array}$ & $\begin{array}{l}\text { Production of Tritium on } \mathrm{C} \\
\mathrm{Al} \text {, and } \mathrm{Fe} \text { by } 130 \text { to } 6 \in 0 \\
\mathrm{MeV} \text { Protons }\end{array}$ \\
\hline $11 \mathrm{~B}$ & $\begin{array}{l}\text { Cross Sections for the }(p, n) \\
\text { Reaction on } 11 \text { B Between } \\
\text { MeV and } 3 \mathrm{GeV}\end{array}$ \\
\hline & $\begin{array}{l}\text { High-Energy Accelerator } \\
\text { Shield Leakage Neutron } \\
\text { Spectra }\end{array}$ \\
\hline
\end{tabular}

Literature Survey of Radiochemical Cross-Sections Data Below $425 \mathrm{MeV}$

$238 \mathrm{U}$

$$
\begin{aligned}
& \mathrm{Er} \\
& 181 \mathrm{Ta}
\end{aligned}
$$

$B i, U$

Np

$239 \mathrm{Pu}$

197Au
Interaction of Fast Protons with Heavy Nuclei

Nuclear Reactions on Ir, Ta, and $\mathrm{Er}$, Induced by Protons with Energies up to $85 \mathrm{MeV}$

Fission of Heavy Elements Induced by Medium High-Energy Protons

Fissionability of Nuclei in the Region Z2/A > 35 by HighEnergy Protons

Angular Distributions of Gamma Rays Emitted in i97 $\mathrm{Au}(\mathrm{p}, 2 \mathrm{n}) 196 \mathrm{Hg}$ and $197 \mathrm{Au}(\mathrm{p}, 4 \mathrm{n}){ }^{194} \mathrm{Hg}$ Reactions

A Literature Surrey of Nonelastic Reactions for Nucleons and Pions Incident on Complex Nuclei between $20 \mathrm{MeV}$ and $33 \mathrm{GeV}$
Yadern Fiz 5,34

(1.967) V.N.Mekhedov

Compt Rend B263,833

(1966) L.Sklavenitis

neutrons

Trans Amer Nucl Soc

neutrons, spectra

9,354(1966)

K.O'Brien et al

Oak Ridge report

ORNL-3884(1966)

H.W.Bertini et al

Izv Akad Nauk SSSR

Ser Fiz 30, 322

(1966)V.S.Barashenkov

et al

Izv Akad Nauk SSSR

Ser Fiz 30,232(1966)

V.S.Barashenkov et al

Z Naturforsch A21, 995(1966) A.C.Pappas

Yadern Fiz 4,97(1966)

V.A.Konshin et al

Lawrence Livermore report UCRL-16987

(1966) T.Yamazaki et al

Oak Ridge report

ORNL-3455(1963)

H.W.Bertini
Monte Carlo method

fission

gamma radiation

data compilation 1955-1962 
C, $160 \quad$ Quasi-Free Ptoton Scattering
at $160 \mathrm{MeV}$

Be,Sn, U

$12 \mathrm{C}$

$12 \mathrm{C}, \mathrm{Li}$

$56 \%$ e, $54 \mathrm{Mn}$

${ }^{9} \mathrm{Be}, 12 \mathrm{C}$

$58,60 \mathrm{Ni}$

$12 \mathrm{C}$

$\mathrm{Si}$

$\mathrm{Si}$
Neutron Production in Thick rargets Bombarded by High Energy Protons

Nuclear Recoil in the $12 \mathrm{C}$ (p.pn) 11 C Rexction I. Angular Distribution

Formation of the Isotopes of Lithium, Beryllium, and Boron by the Interaction of MediumEnergy Protons with $12 \mathrm{C}$ and 160. Application to the Nucleogenesis of Light Elements

Monte Carlo Calculations of Radionuclide Production in Iron Targets Bombarded with 400-MeV Protons

Elastic Scattering of 45-MeV Prouvinis

On High Energy Particle Scattering on Nuclei

Radiaiicn Damage in Silicon. Semiannual Report No. :, Oct. 15, 1963-April 15, 1964

Low-Energy Proton Damage Effects in Silicon and Gallium Arsenide Solar Cells

Fission Cross-Sections of $181 \mathrm{Ta}, \mathrm{Re}, \mathrm{Pt},{ }^{197} \mathrm{Au}, \mathrm{Pb}, \mathrm{Bi}$, Th, 235U, and 238U for Protons with Energy of $150-660 \mathrm{MeV}$
Proc. Williamsburg Conf on Intermediate Energy Physics V.II pp. 700-718(1966) B.Gottschalk et al

Canadian Assoc of Physicists Congress, Vancover,(ND) AED-CONF-65-288-9 J.S.Fraser et al

Phys Rev B145,754 (1966) J.A.Panontin et al

Annales de Physique (Paris)13,791 (1965) E.Gradsztajn

J Geophysical Res 71,1445(1966)

D.B.Ebeoglu et al

UCLA Dept of Phys Report AD-626656 (1965) H.Willmes

Dubna report JINR-P-2431(1965)

B.N.Kalinkin

NASA report N-6519886,NASA-CR-57505 (1964) N.Almeleh et al

NASA report N-6516347, NASA-TX-X54990(1965) D.M.Brown

ne affiliation information STLPUE/101 (V.2)(ND) V.A.Kon'shin tti al model radiation

optical model

Monte Carlo method

effects 


\begin{tabular}{|c|c|c|c|}
\hline $\mathrm{Li}, \mathrm{Be}, \mathrm{B}, \mathrm{C}$ & $\begin{array}{l}\text { Quasi-Free Proton-Proton } \\
\text { Scattering in Light Nuclei } \\
\text { at } 460 \mathrm{MeV}\end{array}$ & $\begin{array}{l}\text { Nucl Phys 79,321 } \\
\text { (1966) T.Berggren } \\
\text { et al }\end{array}$ & spectra \\
\hline C & $\begin{array}{l}\text { Measurement of the Absolute } \\
\text { Differential Cross Section in } \\
\text { Proton-Carbon Elastic Scat- } \\
\text { tering at } 145 \mathrm{MeV}\end{array}$ & $\begin{array}{l}\text { Nucl Phys 79,297 } \\
\text { (1966) O.N.Jarvis, } \\
\text { G.F.Cox }\end{array}$ & scattering \\
\hline \multirow[t]{2}{*}{$\begin{array}{l}{ }^{12} \mathrm{C} C{ }^{27} \mathrm{Al} \\
{ }^{297} \mathrm{Au} \\
{ }^{209} \mathrm{Bi}\end{array}$} & $\begin{array}{l}\text { An Experimental Survey of } \\
\text { Nuclear Reactions Induced by } \\
57 \text { MeV Protons. Part II. } \\
\text { (p,d) Reactions }\end{array}$ & $\begin{array}{l}\text { J Phys Soc Japan } \\
\text { 20,2118(1965) } \\
\text { Y.Ishizaki et al }\end{array}$ & spectra \\
\hline & $\begin{array}{l}\text { Proposed Method for Microgram } \\
\text { Surface Density Measurements } \\
\text { by Observation of Proton- } \\
\text { Produced X Rays }\end{array}$ & $\begin{array}{l}\text { J Applied Physics } \\
\text { 37,564(1966) } \\
\text { J.M.Khan et al }\end{array}$ & $x$-radiation \\
\hline $\mathrm{Ge}$ & $\begin{array}{l}\text { Modifications of the Density } \\
\text { and Mobility of Majority } \\
\text { Carriers in N-Type Germanium } \\
\text { Bombarded by Protons at } 600 \mathrm{MeV}\end{array}$ & $\begin{array}{l}\text { Compt Rend 261,5416 } \\
\text { (1965) D.Daspet } \\
\text { et al }\end{array}$ & $\begin{array}{l}\text { Hall effect, } \\
\text { radiation effects }\end{array}$ \\
\hline \multirow[t]{2}{*}{${ }^{12} \mathrm{C}$} & $\begin{array}{l}\text { The }{ }^{12} \mathrm{C}(\mathrm{p}, \mathrm{pn}) 11 \mathrm{C} \text { Reaction } \\
\text { from } 50 \text { to } 160 \mathrm{MeV}\end{array}$ & $\begin{array}{l}\text { Nucl Phys } 78,476 \\
\text { (1966) D.F.Measday }\end{array}$ & \\
\hline & $\begin{array}{l}\text { Passage of High-Energy Par- } \\
\text { ticles in Matter-Nuclear } \\
\text { Cascades Induced in Dense } \\
\text { Media by 1- and 3-GeV Protons }\end{array}$ & $\begin{array}{l}\text { Brookhaven Lab } \\
\text { report BNL-8721 } \\
\text { (1964) S.P.Shen }\end{array}$ & $\begin{array}{l}\text { cascade showers, } \\
\text { fast neutrons }\end{array}$ \\
\hline${ }^{12} \mathrm{C}$ & $\begin{array}{l}\text { Optical Potentials and High- } \\
\text { Energy lnelastic Proton Scat- } \\
\text { tering }\end{array}$ & $\begin{array}{l}\text { Oak Ridge report } \\
\text { ORNL-P-960(1964) } \\
\text { R.M.Haybron }\end{array}$ & $\begin{array}{l}\text { inelastic } \\
\text { scattering, } \\
\text { optical model }\end{array}$ \\
\hline $\begin{array}{l}27 \mathrm{Al}, 40 \mathrm{Ca} \\
12 \mathrm{C}, 59 \mathrm{Co}\end{array}$ & $\begin{array}{l}\text { Quasi-Free Proton-Proton } \\
\text { Scattering in Light Nuclei } \\
\text { at } 460 \mathrm{MeV}\end{array}$ & $\begin{array}{l}\text { Univ of Chicago } \\
\text { report AD-624318 } \\
\text { (1964) H.Tyren et al }\end{array}$ & spectra \\
\hline $\mathrm{Be}$ & $\begin{array}{l}\text { Electron Bremsstrahlung from } \\
\text { Proton-Excited Targets }\end{array}$ & $\begin{array}{l}\text { Phys Rev } 142,50 \\
\text { (1966) W.T.Ogie: } \\
\text { et al }\end{array}$ & $\mathrm{x}$-radiation \\
\hline $\mathrm{Si}$ & $\begin{array}{l}\text { Calculation of the Density of } \\
\text { Elastic Atomic Displacements } \\
\text { Produced in Silicon and } \\
\text { Germanium by Protons at } 30 \\
\text { MeV } 101 \mathrm{BeV}\end{array}$ & $\begin{array}{l}\text { Compt Rend 261,4709 } \\
\text { (1965) Y.G.De } \\
\text { Lafond et al }\end{array}$ & $\begin{array}{l}\text { radiation } \\
\text { effects }\end{array}$ \\
\hline
\end{tabular}

Nucl Phys $\underline{79,321}$ spectra

(1966) T.Berggren

Nucl Phys 79,297

(1966) O.N Jarvis, G.F.Cox

J Phys Soc Japan

$20,2118(1965)$

Y.Ishizaki et al

J Applied Physics

37,564(1966)

JM.Khan et al

Compt Rend 26I,5416

1965) D.Daspet

Majonity

Carriers in N-Type Germanium

The ${ }^{12} \mathrm{C}(\mathrm{p}, \mathrm{pn}){ }^{11} \mathrm{C}$ Reaction

from 50 to $160 \mathrm{MeV}$

Nucl Phys $\underline{78}, 476$

Brookhaven Lab

report BNL-8721

(1964) S.P.Shen

fast neutrons

inelastic

scattering

ORNL-P-960)(1964)

Univ of Chicago

report AD-624318

Phys Rev 142,50

(1966) W.T.Ogie:

et al

Compt Rend 261, 4709

(1965) Y.G.De

effects 
$\mathrm{Fe}$

$181 \mathrm{Ta}$

$235,238 \mathrm{U}$

$12 \mathrm{C}$

Si

$197 \mathrm{Au}$

$209 \mathrm{Bi}$

$\mathrm{Fe}$

$\mathrm{Ge}$

$\mathrm{Si}$
Calculation of the NucleonMeson Cascade in Iron Initiated by $1-$ and 3-GeV Protons and Comparison with Experiment

Cross Sections for the Fission of $181 \mathrm{Ta}, \mathrm{Re}, \mathrm{Ph} 197 \mathrm{Au}, \mathrm{Pb}$, $209 \mathrm{Bi}, 232 \mathrm{Th}, 235 \mathrm{U}$, and $238 \mathrm{U}$

by Protons with Energies 150 to $660 \mathrm{MeV}$

Numerical Differential Cross Sections of Some Reactions on $12 \mathrm{C}$ Induced by $54.9 \mathrm{MeV}$ Protons

The Effect of High-Energy Protons on Semiconductor Detectors of Nuclear Radiation. II. Diffusion-Drift Detectors

Fluctuations of Energy Loss by Heavy Charged Particles in Silicon Detectors: Preliminary Measurements

Evaporation of Charged Particles from Heavy Nuclei

Calculation of the NucleonMeson Cascade in Iron Initiated by 1 - and 3-GeV Protons and Comparison with Experiment

Some Experimental Data on the Nuclear Cascade in Thick Absorbers

Studies of High-Energy Electron (10-60 MeV) and Proton (20-130 MeV) Radiation Damage in Silicon and Germanium.

Effects of Proton Irradiation on Silicon. Radiation Damage in Semiconductors
Oak Ridge report

ORNL-3852(1965)

R.G.Alsmiller Jr.,

J.Barish

Yadern Fiz 2,682 (1965) V.A.Konshin

et al

Tokoyo report

INSJ-88(1965)

K.Baba et al

Cosmic Res. 3,499

(1965)English vers of

Kosmicheskie Issledovaniya

L.S.Brykina et al

Nucl Instr \& Meth

37, 176(1965)

H.D.Maccabee,

M.R.Raju

Nucl Phys $\underline{75}, 641$

(1966) M.Lefort.

R.Da Silveira

Oak Ridge report

ORNL-P-1733(1965)

R.G.Alsmiller,

J.Barish

NASA report

NASA-SP-71(19ND)

B.S.P.Shen

Radiation Damage

in Semiconductors

(Pub. Dunod) Paris

(1965)p.407

J.C.Corelli et al

Radiation Damage

in Semiconductors

(Pub.Dunod) Paris

(1965)p.79 A.Authier, Montenay-Garestier cascade showers

fission data

inelastic scattering

radiation effects

slowdown

spectra

nuclear cascade, spectra

cascade showers

radiation effects

$x$ radiation 
Al

$9 \mathrm{Be}, 12 \mathrm{C}$

$6 \mathrm{Li}$

$\mathrm{Ge}, \mathrm{Si}$

$28 \mathrm{Si}$

$197 \mathrm{Au}$

U

$\mathrm{Bi}$

Al,Si

$\mathrm{Bi}, \mathrm{Au}$

$\mathrm{Ta}, \mathrm{Sn}$

$238 \mathrm{U}$
A Precise Mieasurement of the Range of 100-MeV Protons in Aluminum

Study of the Inelastic Scattering of Protons at $155 \mathrm{MeV}$ on Light Nuclei

A Calculation of Proton Induced Damage in Germanium and Silicon

Yield and Angular Distribution of Secondary Nucleons from Flat Shielding under the Action of $660-\mathrm{MeV}$ Protons

Cascade Mechanism of the High Energy Nuclear Reactions I. Total Inelastic Cross Sections, Angular and Energy Distribution of the Fast Particles

Study of Nuclear Fission Asymmetry and Anisotropy During Irratiation of Uranium and Bismuth by High-Energy Protons

Charged Particle Radiation Damage in Semiconductoss. XI. Effects of Low-Energy Protons and High-Energy Electrons on Silicon. Interim Technical Final Report

Measurement of Absolute Cross Sections of Fission Induced by $156 \mathrm{MeV}$ Protons, using Mica as a Fission Fragment Detector

Recoil Properties of Fragments in 150-MeV Proton Fission of Uranium-238. II. Momentum Transfer and Excitation Energy
Can J Phys 43,1904

(1965) P.M.Portner, R.B.More

Cahiers Phys 173,1 (1965) J Jacmart

Rensselaer Polytech.Instit.report NP-15463(1964)

E.A.Saunders, Thesis

Obnisk Fiziko-

Energeticheskii

Institut report

NP-15385(1965)

V.A.Kon'shin et al

Sov J Nucl Phys $\underline{1}$, 234(1965)

F.P.Denisov et al

Radievyi Institut Leningrad, Thesis (1965) A.I.Obukhov

TRW Space Tech Labs report NP-15090 (1965) J.R.Carter, R.G.Downing

Symp Phys \& Chem of Fission, Salzburg, Austria(1965) M.Maurette, C.Stephan

J Inorg \& Nucl Chem 27,959(1965) V.E.Noshkin, Jr., T.T.Sugihara
Monte Carlo

slowdown

scattering

radiation effects

shielding

cascade theory, spectra

evaporation

Hall effect

fission method 
$238 \mathrm{U}$

$12 \mathrm{C}, \mathrm{O}$

$\mathrm{N}$

Ta,Ir

$238 \mathrm{U}$

Al,C

Al, $12 \mathrm{C}$

160

$\mathrm{Al}, \mathrm{Bi}, \mathrm{C}$

Co

$\mathrm{Ge}$
Recoil Properties of Fragments in 150-MeV Proton Fission of Uranium-238. I. Ranges and Kinetic Energies

Knock-Out of Alpha Particles from Lithium, Oxygen and Nitrogen Nuclei by $660 \mathrm{MeV}$ Protons

Nuclear Reactions on Ir, Ta, and Er Induced by Protons of Energy up to $85 \mathrm{MeV}$

Interactions of Fast Protons with Heavy Nuclei

A Monte Carlo Study of Neutron Transport Through Aluminum, Polyethylene, and Carbon Slabs

Calculation of Proton Induced Gamma Ray Spectrum and Comparison with Experiment

Spectra of Gamma Rays Produced by the Interaction of Approximately 160-MeV Protons with $\mathrm{Be}, \mathrm{C}, \mathrm{O}, \mathrm{Al}, \mathrm{Co}$, and $\mathrm{Bi}$

Damage to Germanium Due to 22 and $40-\mathrm{MeV}$ Proton Bombardments
$J$ Inorg \& Nucl Chem 27,943(1965)

V.E.Noshkin,Jr., T.T.Sugihara

Bull Acad Sci USSR

Phys Ser 29,239

A.P.Zhdanov et al

Dubna report JNR-1978(1965)

V.S.Barashenkov et al

Dubna report JINR-P-1969(1965)

V.S.Barashenkov et al

Radiation Research Associates report RRA-T47(1964) M.B.Wells

2nd Symp on Protection against Radiation Hazards in Space Gatlinburg,Tenn.,CONF720-13(1964)C.W.HII, K.M.Simpson Jr

Oak Ridge report ORNL-3506(1965) W.Zobel et al

NASA report

NASA-TN-D-2727 (1965)R.A.Breckenridge, C.Gross fission products

spectra

evaporation

fast neutrons,

Monte Carlo method

recoils

radiation

effects 
$\mathrm{Al}, \mathrm{Be}, \mathrm{Bi}$

C.Co

$238 \mathrm{U}$

Al,Be,Bi

C, $\mathrm{Co}$

Al,Be,Bi

C,Co,O

Au

$\mathrm{Si}$

Si

, Ga

$\mathrm{Si}$
Experimental Studies for High-

Energy Radiation Shielding

Theoretical Studies of High-

Energy Radiation Shielding

Studies on the Range of $140 \mathrm{Ba}$ in Ptoton-Induced Fission of 238U and a Semi-Empirical Derivation of a General RangeEnergy Relation

Differential Cross Sections by Flight-Time Spectroscopy for Proton Production in Reactions of $160-\mathrm{MeV}$ Protons on Nuclei

Spectra of Gamma Rays Produced by Interaction of Approximately to $160 \mathrm{MeV}$ Protons with $\mathrm{Be}, \mathrm{C}, \mathrm{O}, \mathrm{Al}, \mathrm{Co}$, and $\mathrm{Bi}$

Application of Two-Body Kinematics to Recoil Studies of High-Energy Nuclear Reactions

Low-Rate Proton Bombardment of Diffused Silicon Counters

Proton Bombardment Damage in Silicon

Results of Low-Energy Proton Irradiations of Solar Cells
Oak Ridge report ORNL-3714 (V.1)

spectra p.119-33(19ND)

no author

ORNL-3714(V.1) p.9i-118(19ND) no author

Can J Chem 43,232 (1965) S.Mukherji,

fission products L.Yaffe

Oak Ridge report ORNL-P-848(1964)

R.W. Peelle et al

charged-particle spectra

Oak Ridge report

ORNL-P-813(1963)

W.Zobel et al

Phys Rev $137, B 848$

(1965) J.B.Cumming

Nucl Instr Meth $\underline{32}$, 171(1965) R.E.Scott

Physica Status

Solidi (E.Ger.)

8, 105(1965)

R.Bubakova, Z.Szmid

CONF-657-6(1964)

4th Annual Photo-

voltaic Spec. Conf.

Cleveland June 1964

E.A.Lodi et al recoils

radiation effects

gamma radiation

radiation effects

radiation effects 
\begin{tabular}{ll}
$\mathrm{Si}$ & $\begin{array}{l}\text { Proton and Electron Irradia- } \\
\text { tions of Contoured Semicon- } \\
\text { ductor Radiation Detectors }\end{array}$ \\
$\mathrm{C}, \mathrm{Au}, \mathrm{Pb}$ & $\begin{array}{l}\text { Neutron Yields from Proton } \\
\mathrm{Mg}, \mathrm{Si}\end{array}$ \\
\hline
\end{tabular}

$12 \mathrm{C}$

C

$12 \mathrm{C}$

$232 \mathrm{Th}$

$\mathrm{Ge}$

Si
$12 \mathrm{C}, 14 \mathrm{~N}$
Determination of the Cross Sections of Some Nuclear Reactions Occurring as a Result of Cosmic Radiation

Excitation Functions of (p,pn) and $(p, 2 p 2 n)$ Reactions in Light Nuclei between 15 and $155 \mathrm{MeV}$

Radiochemical Studies of Charge and Mass Distribution in the Light Fragment Region in Fission of Uranium Induced by $170 \mathrm{MeV}$ Protons

Proton Energy-Loss Distributions from Thin Carbon Films

Distorted IVave Effects and the Impulse Approximation in the Inelastic Scattering of $147 \mathrm{MeV}$ Protons from $12 \mathrm{C}$

Spallation Nuclear Reactions Induced by $150 \mathrm{MeV}$ Protons on Thorium

Separation and Identification of the Products of Germanium Spallation by High Energy Protons

Spectrometry of Heavy, Intermediate-Energy Charged Particles, Using N-I-P Type Silicon Detectors
General Elec.Co. report NYO-3246-3 (1964) J.B.Trice et al

Nucl Instr Meth 30, 73(1964)

R.R.Borchers et al

$\mathrm{J}$ de Physique (Paris) 25,704 (1964) L.Valentin et al

J Inorg \& Nucl Chem 26, 1639(1964)

E. Hageboe et al

Phys Rev 135,A1436 (1964) A.L.Morsell

Nucl Phys 54,193 (1964) D.J.Rowe et al

Univ of Paris, Thesis NP-13838(1961) G.Simonoff

impulse approximation

Vestn.Leningr. Univ No.4, Ser Fiz I Khim 1, 105(1964)

A.N.Murin et al

At Energ 16,360

spectra

electric

properties

spectra

fission products

losses

evaporation

spallation (1964) O.A.Matveev et al 


\begin{tabular}{|c|c|c|c|}
\hline $9 \mathrm{Be}, 7 \mathrm{Li}$ & $\begin{array}{l}\text { Neutrons from the Proton } \\
\text { Bombardment of } 6 \mathrm{Li}, 7 \mathrm{Li},{ }^{9} \mathrm{Be} \text {, } \\
11 \mathrm{~B} \text {, and } 18 \mathrm{O}\end{array}$ & $\begin{array}{l}\text { Nucl Phys 53,209 } \\
\text { (1964) J.K.Bair et al }\end{array}$ & \\
\hline $\mathrm{Fe}$ & $\begin{array}{l}\text { Radionuclide Productions in } \\
\text { Thick Iron Targets Bombarded } \\
\text { with 1- and 3-GeV Protons }\end{array}$ & $\begin{array}{l}\text { J Geophysical Res } \\
\text { 69,2231(1964) } \\
\text { J.P.Shedlovsky, } \\
\text { G.V.S. Rayudu }\end{array}$ & radioisotopes \\
\hline $\begin{array}{l}\text { Gd,Ta,Tm } \\
W, Y b\end{array}$ & $\begin{array}{l}\text { Elastic Scattering of } 55 \mathrm{MeV} \\
\text { Protons by Heavy Nuclei }\end{array}$ & $\begin{array}{l}\text { Phys Lett } 9,332 \\
\text { (1964) H.Kamitsubo } \\
\text { et al }\end{array}$ & elastic scattering \\
\hline $\mathrm{Al}$ & $\begin{array}{l}\text { Perturbation Theory for the } \\
\text { Creation of Deuterons from } \\
\text { High Energy Proton Bombardment } \\
\text { of Matter }\end{array}$ & $\begin{array}{l}\text { Phys Lett } 9,330 \\
\text { (1964) S.T.Butler. } \\
\text { C.A.Pearson }\end{array}$ & \\
\hline $\mathrm{Be}, \mathrm{Li}$ & $\begin{array}{l}\text { Formation of Multiply Charged } \\
\text { Particles by the Interaction } \\
\text { of } 75-\text { to } 350-\mathrm{MeV} \text { Protons } \\
\text { with Nuclei }\end{array}$ & $\begin{array}{l}\text { Zh Eksperim I Teor } \\
\text { 46,809(1964) } \\
\text { M.M.Makarov }\end{array}$ & \\
\hline \multirow[t]{2}{*}{$\mathrm{Bi}, \mathrm{Au}$} & $\begin{array}{l}\text { Emission of } 3 \mathrm{He} \text { and } 4 \mathrm{He} \text { from } \\
\text { Heavy Nuclides Bombarded by } \\
155 \mathrm{MeV} \text { Protons }\end{array}$ & $\begin{array}{l}\text { Phys Let: } 9,146 \\
\text { (1964) H.Dubost } \\
\text { et al }\end{array}$ & \\
\hline & $\begin{array}{l}\text { Formation Cross Sections of } \\
\text { Varicus Radionuclides from } \mathrm{Ni} \text {, } \\
\mathrm{Fe}, \mathrm{Si}, \mathrm{Mg}, \mathrm{O} \text {, and } \mathrm{C} \text { for } \\
\text { Protons of Energies Between } \\
130 \text { and } 400 \mathrm{MeV}\end{array}$ & $\begin{array}{l}\text { Can J Chem 42,1149 } \\
\text { (1964) G.V.S.Rayudu }\end{array}$ & evaporation \\
\hline $\mathrm{C}, \mathrm{Cu}$ & $\begin{array}{l}\text { Characteristics and Intensity } \\
\text { Profile of a High-Energy- } \\
\text { Proton Beam after Scattering } \\
\text { in a Thick Target }\end{array}$ & $\begin{array}{l}\text { Lawrence Berkeley } \\
\text { report UCRL-11184 } \\
\text { (19ND) R.Wallace } \\
\text { et al }\end{array}$ & radiation effects \\
\hline $\mathrm{Al}, \mathrm{C}$ & $\begin{array}{l}\text { The Energy Spectra and Angular } \\
\text { Distribution of } 8 \mathrm{Li} \text { Fragments } \\
\text { Produced in the Interaction } \\
\text { between } 660 \mathrm{MeV} \text { Protons and } \\
\text { Aluminum Nuclei }\end{array}$ & $\begin{array}{l}\text { Zh Eksperim I Teor } \\
\text { Fiz } 46,431(1964) \\
\text { V.I.Bogatin et al }\end{array}$ & \\
\hline
\end{tabular}


C

$\begin{array}{ll}\mathrm{Ai}, \mathrm{Pb}, \mathrm{U} & \text { Neutron Production by } 450-\mathrm{MeV} \\ \text { Protons }\end{array}$

$27 \mathrm{Al}, 52 \mathrm{C}$

$59 \mathrm{Co}$

$9 \mathrm{Be}$

$40 \mathrm{Ca}, 12 \mathrm{C}$

$10,11_{B}$

$\mathrm{C}, \mathrm{Fe}$

$28 \mathrm{Si}, 32 \mathrm{~S}$

$\mathrm{Au}, \mathrm{U}$

$\mathrm{N}, \mathrm{Pt}$

$\mathrm{Ta}, \mathrm{V}$

$209 \mathrm{Bi}$

$238 \mathrm{U}$

The Measurement of the Total Proton Reaction Cross-Section of Carbon in the Energy Region up to $50 \mathrm{MeV}$

Protons

Inelastic Scattering of 155

$\mathrm{MeV}$ Protons on $23 \mathrm{Na}, 27 \mathrm{Al}$, $31 \mathrm{P}, 39 \mathrm{~K}, 48 \mathrm{Ti}, 52 \mathrm{Cr}, 56 \mathrm{Fe}$. and $59 \mathrm{Co}$

Inelastic Scatteing of 155

$\mathrm{MeV}$ Protons on Light Nuclei

Spallation Cross Sections for Long-Lived Radionuclides in Iron and Light Nuclei

Inelastic Scattering of 155 $\mathrm{MeV}$ Protons on $28 \mathrm{Si}, 32 \mathrm{~S}$, and $40 \mathrm{Ca}$

Cascades by the Monte Carlo de Physique

(Paris) 24,1052

(1963)

W.J.Homyak

$\mathrm{J}$ de Physique

(Paris) 24,901

(1963)

L.Kowalski,

C.Stephan

J Phys (Paris)

24,839(1963)

R.Klapisch et al

$\mathrm{J}$ de Physique

(Paris) 24,829

(1963) E.Graद-

sztajn

ethod. Protons of 82, 110 ,

and $156 \mathrm{MeV}$ on $238 \mathrm{U}, 209 \mathrm{Bi}$, spectra

inelastic

scattering, neutron sources

inelastic

scattering

spallation

scattering

fission

Monte Carlo method $197 \mathrm{Au}, 140 \mathrm{Ce}$, and $109 \mathrm{Ag}$ 
6,7 Li Elastic Scattering of Protons at $155 \mathrm{MeV}$ on $6 \mathrm{Li}$ and $7 \mathrm{Li}$

$27 \mathrm{Al}, 12 \mathrm{C}$

$235,238 \mathrm{U}$

$\mathrm{Al}, \mathrm{Be}, \mathrm{C}$ W,V

At

Al,Be,Bi

C.Co

Al,Bi,C

$\mathrm{Co}, \mathrm{Cu}$

$27 \mathrm{Al}, 12 \mathrm{C}$

$65 \mathrm{Cu}$

$\mathrm{Fe}$
Monitor Reactions for High

Energy Proton Beams

Fragment Kinetic Energy and Mass Distriburion for Fission of Uranium Bombarded by $\mathbf{5 5 . 3}$ $\mathrm{MeV}$ Protons

Sputtering of Metals by Hydrogen Ions
Phys Lett 8,54

(1964)

B.Tatischeff et al

Ann Rev Nucl Sci

13,261(1963)

J.B.Cumming

Genshikaku Kenkyu

(Japan) $\underline{8}, 356$

(1963) S.Shirato,

M.Seki

J Applied Phys

35,322(1964)

C. Z.Kenknight,

G.K.Wehner

Bull Acad Sci USSR

Phys Series ??

1270(1963) L.P.Moskaleva,

A.K.Lavrukhina

Oak Ridge report

ORNL-3499(19ND)

J.W.Wachter et al

Oak Ridge report

ORNL-3499(V.II)

p.73 (19ND)

R.W. Peelle et al

Oak Ridge report

ORNL-3499(V.II)

p.31(19ND)

H.W.Bertini impulse

approximation

fission products absorption, particle tracks neutrons, recoils 
$12 \mathrm{C}$

$\mathrm{Al}, \mathrm{Be}, \mathrm{Cu}$

$\mathrm{Si}$

$A u, U$

$27_{\mathrm{Al}}, 12 \mathrm{C}$

$\mathrm{Al}, \mathrm{Cu}, \mathrm{W}$

$\mathrm{Al}, \mathrm{C}, \mathrm{Fe}$

$\mathrm{Si}$

27 Al
Decay of Residual Nuclei

Produced in the Interaction

of $660-\mathrm{MeV}$ Protons with Carbon

Nuclei

Activation of Various

Materials by Irradiation with

660-MeV Protons

Charged Particle Radiation

Damage in Semi-Conductors.

IV. High Energy Froton Radia-

tion Damage in Solar Cells

Kinematic Effect in Fission

Induced by $156 \mathrm{MeV}$ Protons in

the Nuclei of $U, B i$, and $A U$

Determination of the Inten-

sities of Proton Beams from

40 to $150 \mathrm{MeV}$

The Evaporation of Neutrons

from Nuçei Bombarded with

High Energy nuvins

Contribution to the Study of

Double Indirect Pickup:

Measurement of Tritium Produc-

tion by $92-$ and $105-\mathrm{MeV}$ Protons

on Seve: al Targets

Charged Particle Radiation

Damage in Semiconductors. I.

Experimental Proton Iradi-

ation of Sclar Cells. Final

Report

High-Energy Nuclear Reaction

Cross-Sections. Part II
Zh Eksperim I Teor

Fix 45,455(1963)

Z.P.Zhdanov,

P.I.Fedotov

At Energ 15,126

(1963) M.M.Komochkov

Space TechLabs.

Inc. report NP-13031

(1963)J.M.Denney et al

radiation effects

fission products

(1962) L.Kowalski

et al

$J$ de Physique

23,371(1962)

C.Brun et al

Nucl Phys 35,177

(1962) D.M.Skyme

neutron flux

J de Physique

23, 167(1962)

spectra

C.Brin et al

Space Tech.Labs

Inc. report NP-11655

(1961) J.M.Denney,

R.G.Downing

radiation effects
CERN report

CERN-62-9(1962)

E.Bruninx 
Table 1 (Cont.)

Si

Proton Damage to Solar Cells

$\mathrm{Pb}, \mathrm{Li}, \mathrm{U}$

Neutrons Emitted at 00 from

Nuclei Bombarded by $143 \mathrm{MeV}$

Protons

$\mathrm{Bi}$

$\mathrm{Al}, \mathrm{C}$

$\mathrm{Cu}, \mathrm{Pb}$

$\mathrm{C}, \mathrm{Cu}, \mathrm{Ni}$

C

$\mathrm{Na}, \mathrm{Mg}, \mathrm{Al}$

Si.P.S

$\mathrm{Au}, \mathrm{Mn}$

$197 \mathrm{Au}$

$\mathrm{J}, \mathrm{C}$
Secondary Electron Emission from Clean Metal Surfaces

Bombarded by Fast Hydrogen

Ions

The Question of the Product

Yield of the Deep Spallation

Reaction 209Bi(p,xn, yp)

Measurement of Protu? Inelastic Cross Sections Between 77

$\mathrm{MeV}$ and $133 \mathrm{MeV}$

Optical-Model Analysis of Elastir. Scattaring of Protons of Carbon at Intermediate Energies

Gimma Radiaticn from Nedium Energy Bombardment of Sodium, Magnesium. Alumınum, Silicon, Phosphorus, and Sulphur

Reactions (p,pn) at Medium Energies

Proton, Deuteron, Triton, and Helium Nuclei Emission by 197 Au Bombarded with 154-MeV Protons

The Elastic Scattering of 180 MeV Protons from Nuclei
Lockheed Co.report

LMSD-703735-1

(1961) K.T.Chow,

E.A.Lodi

Nucl Phys $\underline{30,475}$

(1962) P.H.Bowen

et al

Izvest Akad Nauk

USSR Ser Fiz 26,245

(1962) A.V.Kalyamin

et al

Nucl Phys 29,474

(1962) R.Goloskits,

K.Strauch

Proc Phys Soc

(London) 79,148

(1962) L.N.Large,

W.S.Whitlock

Phys Rev A125,975

(1962) J.S.Nodvik

et al

Nucl Phys 37,23(1962)

K.T.Foley et al radiation effects

spectra

optica! model

emission,

secondary emission

uptical model

gamma radiation, direct reactions
J de Physique 22 ,

636(1961)

M.Gusakow et al

$\mathrm{J}$ de Physique 22

615(1961)

J.Genin et al

Arkiv Fysik 19,541

(1961) A.Hohansson

et al spectra

optical model 
Table I (Cont.)

\begin{tabular}{|c|c|c|c|}
\hline $\begin{array}{l}\mathrm{Al}, \mathrm{Be} \\
\mathrm{Ca}, \mathrm{C}\end{array}$ & $\begin{array}{l}\text { Tctal Nucleir Reaction Cross } \\
\text { Sections for } 180 \mathrm{MeV} \text { Protons }\end{array}$ & $\begin{array}{l}\text { Arkiv Fysik } 19,527 \\
\text { (1961) AJohansson } \\
\text { et al }\end{array}$ & nuclear models \\
\hline $6,7 \mathrm{Li}$ & $\begin{array}{l}\text { Quasi-Elastic Interaction of } \\
155-\mathrm{MeV} \text { Protons with Protons } \\
\text { in } 6 \mathrm{Li} \text { and } 7 \mathrm{Li}\end{array}$ & $\begin{array}{l}\text { Phys Rev Lett } 7 \text {. } \\
261(1961) \\
\text { J.P.Garron et al }\end{array}$ & spectra \\
\hline $\begin{array}{l}\mathrm{Pb} \\
\mathrm{Zn}\end{array}$ & $\begin{array}{l}\text { Production of Tritium in Lead } \\
\text { and Aluminum by High Energy } \\
\text { Protons, Deuterons and Alpha } \\
\text { Particles }\end{array}$ & $\begin{array}{l}\text { Zhur Eksptl I Teor } \\
\text { Fiz } 40,1263(1961) \\
\text { V.V.Kuznetsov }\end{array}$ & \\
\hline $\mathrm{Bi}, \mathrm{U}$ & $\begin{array}{l}\text { Anisotropy in the Fission of } \\
\text { Bismuth and Uranium Irradi- } \\
\text { ated by } 660 \mathrm{MeV} \text { Protons }\end{array}$ & $\begin{array}{l}\text { Zhur Eksptl I Teor } \\
40.1250(1961) \\
\text { A.I.Obukhov, } \\
\text { N.A.Perfilov }\end{array}$ & fission products \\
\hline $\begin{array}{l}\text { Al.Cu } \\
\mathrm{U}\end{array}$ & $\begin{array}{l}\text { High-Velocity Range and } \\
\text { Energy-Loss Measurements in } \\
\mathrm{Al}, \mathrm{Cu}, \mathrm{Pb}, \mathrm{U} \text { and Emulsion }\end{array}$ & $\begin{array}{l}\text { Nuovo Cimento } 19 \\
41(1961) \text { W.H.Barkas, } \\
\text { S.Von Friesen }\end{array}$ & slowdown \\
\hline $\begin{array}{l}\mathrm{Al}, \mathrm{Be} \\
\mathrm{C} . \mathrm{O}\end{array}$ & $\begin{array}{l}\text { Proton Scattering from Light } \\
\text { Elements at } 142 \mathrm{MeV}\end{array}$ & $\begin{array}{l}\text { Nucl Phys } 25,642 \\
\text { (1961) A.E.Taylor, } \\
\text { E.Wood }\end{array}$ & \\
\hline Th & $\begin{array}{l}\text { Spallation Reactions of } \\
\text { Thorium by } 150 \text { and } 82 \mathrm{MeV} \\
\text { Protons }\end{array}$ & $\begin{array}{l}\text { Nucl Phys } 25,216 \\
\text { (1961) MLefort } \\
\text { et al }\end{array}$ & evaporation \\
\hline $\mathrm{Ni}$ & $\begin{array}{l}\text { Emission of Soft Electro- } \\
\text { magnetic Radiation from } \\
\text { Metals by the Impact of } \\
\text { High-Energy Protons and } \\
\text { Positive Ions }\end{array}$ & $\begin{array}{l}\text { Nature } 189,996 \\
\text { (1961) } \\
\text { R.M.Chaudhri, } \\
\text { M.Y.Khan }\end{array}$ & $\begin{array}{l}\text { light, ultra- } \\
\text { violet } \\
\text { radiation }\end{array}$ \\
\hline $\begin{array}{l}\text { Al, Au } \\
\text { In. } \mathrm{Li}\end{array}$ & $\begin{array}{l}\text { The Elastic Scattering of } 160 \\
\text { MeV Protons from Lithium, } \\
\text { Aluminum, Indium and Gold }\end{array}$ & $\begin{array}{l}\text { Nucl Phys 21,383 } \\
\text { (1960) } \\
\text { A.Johansson et al }\end{array}$ & optical model \\
\hline $6,7_{\mathrm{L}, \mathrm{i}}$ & $\begin{array}{l}\text { Elastic Scattering Angular } \\
\text { Distributions of Protons from } \\
6 \mathrm{Li} \text { and } 7 \mathrm{Li} \text { at } 40 \mathrm{MeV}\end{array}$ & $\begin{array}{l}\text { Nuclear Forces and } \\
\text { the Few Nucleon } \\
\text { Problem V II, p. 683-4 } \\
\text { (19ND) S.Chen, } \\
\text { NM.Hintz }\end{array}$ & \\
\hline $12 \mathrm{C}$ & $\begin{array}{l}\text { Quasi-Elastic Scattering of } \\
153 \mathrm{MeV} \text { Protons by P-State } \\
\text { Protons in } 12 \mathrm{C} \text {. I. Experi- }\end{array}$ & $\begin{array}{l}\text { Nucl Phys } 18.46 \\
\text { (1960) TJ.Gooding, } \\
\text { H.G.Pugh }\end{array}$ & $\begin{array}{l}\text { elastic } \\
\text { scattering, } \\
\text { spectra }\end{array}$ \\
\hline
\end{tabular}


Au

Reactions $(p, x n)$ Induced in Gold by Protons at $155 \mathrm{MeV}$

$12 \mathrm{C}$

C

$12 \mathrm{C}$

C

$27 \mathrm{Al}, 12 \mathrm{C}$

$65 \mathrm{Cu}$

$\mathrm{Cu}$

$\mathrm{Bi}, \mathrm{Cu}, \mathrm{Au}$

$6,7 \mathrm{Li}$
Quasi-Elastic Scattering of 153-MeV Protons by Protons in the P State of $12 \mathrm{C}$

Study of Deuterons and Tritons Emitted During Bombardment of Carbon Nuclei by 155-MeV Protons

Study of (p.p gamma) Reactions Iniciated by $155-\mathrm{MeV}$ Protons. The Case of the Giant Resonance

Elastic and Inelastuc Scattering of $155-\mathrm{MeV}$ Frotons on Carbon

Some Cross Sections for the Production of Radio-Nuclides in the Bombardment of $\mathrm{C}, \mathrm{N}$, $\mathrm{O}$, and $\mathrm{Fe}$ by Medium Energy Protons

Radiochemical Studies of the $(p, p n)$ Reaction in Complex Nuclei in the $80-450-\mathrm{MeV}$ Range

Copper Spallation by Protons with an Energy of $680 \mathrm{MeV}$

Monte Carlo Calculations of Nuclear Evaporation Processes V. Emission of Particles Heavier than ${ }^{4} \mathrm{He}$

Total Reaction Cross Sections of Several Nuclei for 61-MeV Protons

Production of Tritium in Thorium by Protons at 135 $\mathrm{MeV}$
J de Physique 21, 343(1960)

N.Poffe et al

J de Physique 21, 326(1960)

TJ.Gooding, H.G.Pugh

J de Physique 21,

322(1960)

P.Radvanyi,

J.Genin

$\mathrm{J}$ de Physique 21,

320(1960)

H.Langevin-

Joliot et al

$\mathrm{J}$ de Physique 2 !.

317(1960)

J.P.Garron et al

elastic and inelastic scattering

Phys Rev 118,1618

(1960) M.Honda, D.Lal

Phys Rev 118,1591 (1960) H.P.Yule,

A.Turkevich

Kernenergie 1,119 (1958) A.K.Lavrukhina et al

Monte Carlo method

Phys Rev 118,791

(1960) L.Dostrovsky

et al

inelastic

scattering

scatering

fission products

Phys Rev 117,1334

(196) V.Meyer et al

$\mathrm{J}$ de Physique 20 ,

959(1959)

M.LeFort et al
Monte Carlo method, nuclear evaporation 


\begin{tabular}{|c|c|c|c|}
\hline Si & $\begin{array}{l}\text { Radiation Damage of Semi- } \\
\text { conductors by Protons }\end{array}$ & $\begin{array}{l}\text { CERN report CERN- } \\
62-5(1962) \\
\text { G.Backenstoss et al }\end{array}$ & radiation effects \\
\hline C & $\begin{array}{l}\text { The Total Proton Reaction } \\
\text { Cross Section of Carbon from } \\
10.68 \mathrm{MeV} \text { by a New Method }\end{array}$ & $\begin{array}{l}\text { Nucl Phys } 13,511 \\
\text { (1959) E.J.Burge }\end{array}$ & spectra \\
\hline C & $\begin{array}{l}\text { Proton Total Reaction Cross } \\
\text { Sections at } 34 \mathrm{MeV}\end{array}$ & $\begin{array}{l}\text { Nucl Phys } 12,241 \\
\text { (1959) TJ.Gooding }\end{array}$ & optical model \\
\hline \multirow[t]{2}{*}{$\mathrm{Be}, \mathrm{C}, \mathrm{U}$} & $\begin{array}{l}\text { Interaction Between } 660 \mathrm{MeV} \\
\text { Protons and Atomic Nuclei and } \\
\text { the Nuclear Internal Momentum } \\
\text { Distribution of Nucleons }\end{array}$ & $\begin{array}{l}\text { Zhur Eksptl. I } \\
\text { Teoret Fiz 36, } 1631 \\
\text { (1959) L.S.Azhgirei } \\
\text { et al }\end{array}$ & spectra \\
\hline & $\begin{array}{l}\text { Range-Energy Measurements for } \\
742-\mathrm{MeV} \text { Protons }\end{array}$ & $\begin{array}{l}\text { Lawrence Berkeley } \\
\text { Lab. report UCRL- } 8762 \\
\text { (1959) Thesis J.E.Ward }\end{array}$ & losses \\
\hline $\begin{array}{l}{ }^{9} \mathrm{Be} \\
10,11_{\mathrm{B}}\end{array}$ & $\begin{array}{l}\text { Total Neutron Yields from } \\
\text { Light Elements Under Proton } \\
\text { and Alpha Bombardment }\end{array}$ & $\begin{array}{l}\text { Phys Rev } 114,571 \\
\text { (1959) J.H.Gibbons, } \\
\text { R.L.Macklin }\end{array}$ & \\
\hline As & $\begin{array}{l}\text { Reactions of } 378 \mathrm{MeV} \text { Protons } \\
\text { with Arsenic }\end{array}$ & $\begin{array}{l}\text { Columbia Univ } \\
\text { Thesis NYO-6141 } \\
\text { (1954) J.B.Cumming }\end{array}$ & $x$-radiation \\
\hline $\mathrm{Be}, \mathrm{C}, \mathrm{W}$ & $\begin{array}{l}\text { Energy-Range Relation of } 660 \\
\text { MeV Frotons }\end{array}$ & $\begin{array}{l}\text { Zhur Eksptl I } \\
\text { Teoret Fiz 36,658 } \\
\text { (1959) V.P.Zrelov, } \\
\text { G.D.Stoletov }\end{array}$ & energy range \\
\hline $\mathrm{Au}$ & $\begin{array}{l}\text { Study of Thallium, Lead, and } \\
\text { Bismuth Nuclei Produced in the } \\
\text { Bombardment of Gold with 380- } \\
\text { MeV Protons }\end{array}$ & $\begin{array}{l}\text { Phys Rev } 113,1125 \\
\text { (1959) A.E.Metzger, } \\
\text { J.M.Miller }\end{array}$ & $\begin{array}{l}\text { fission products, } \\
\text { spectra }\end{array}$ \\
\hline $\mathrm{C}, \mathrm{O}$ & $\begin{array}{l}\text { Total Cross Sections for } 910 \\
\text { MeV Protons }\end{array}$ & $\begin{array}{l}\text { Nucl Phys } 9600(1959) \\
\text { M.E.Law et al }\end{array}$ & \\
\hline U & $\begin{array}{l}\text { The Fission Cross-Section of } \\
\text { Uranium for } 147 \mathrm{MeV} \text { Protons }\end{array}$ & $\begin{array}{l}\text { Harwell Lab report } \\
\text { AERE-N/R-1438(1954) } \\
\text { G.N.Harding }\end{array}$ & \\
\hline C & $\begin{array}{l}\text { Optical Model Aitalysis of the } \\
\text { Scattering of } 310 \mathrm{MeV} \text { Protons } \\
\text { by Carbon }\end{array}$ & $\begin{array}{l}\text { Proc Phjs Soc } \\
\text { (London)73,185(1959) } \\
\text { C.J.Batty }\end{array}$ & optical model \\
\hline
\end{tabular}


$232 \mathrm{Th}$

${ }^{239} \mathrm{Pu}$

$238 \mathrm{U}$

C

$27 \mathrm{Al}, 28 \mathrm{Si} \quad$ Nuclear Recoil Tracks of $24 \mathrm{Na}$

U

$\mathrm{Al}, \mathrm{Pb}$

$\mathrm{Bi}, \mathrm{Pb}$

Be,Th

$\mathrm{U}$

$27 \mathrm{Al}, \mathrm{Cu}$

$27 \mathrm{Al}$

$209 \mathrm{Bi}$

$65 \mathrm{Cu}$

$\mathrm{Mn}, \mathrm{Ni}$ and Mechanism of Nuclear

Reactions $27 \mathrm{Al}(\mathrm{p}, 3 \mathrm{pn})$,

$28 \mathrm{Si}(p, 4 p n)$, and $31 \mathrm{P}(\mathrm{p}, 5 \mathrm{p} 3 \mathrm{n})$

with $660 \mathrm{MeV}$ Protons

rission Processes in the

Proton Bombardment of $232 \mathrm{Th}$,

$238 \mathrm{U}$, and $239 \mathrm{Pu}$

Multi-Charged Particles

Emitted in Spallation of

Carbon Nuclei by $660 \mathrm{MeV}$

Protons

On Triple Spallation of

Uranium Nuclei

Production of Tritium in

Metals under the Action of

120 to $660 \mathrm{MeV}$ Protons

Secondary Nuclear Reactions

in Bismuth and Lead Induced

by High Energy Proton

Bombardment

Neutron Production at High

Energies

Monte Carlo Calculations on

Intranuclear Cascades

II. High-Energy Studies and

Pion Processes

Monte Carlo Calculations on

Intranuclear Cascades

I. Low-Energy Studies

Neutron Yields from Thick

Targets Bombarded by 18- and

32-MeV Protons

Kriock Out of Deuterons from

$\mathrm{Li}, \mathrm{Ee}, \mathrm{C}$ and $\mathrm{O}$ Nuclei by

Protons of Energy $675 \mathrm{MeV}$
Chalk River (Can-

ada) report $A / C o n f$.

15/P/6(ND)

J.P.Butler et al

Zhur Eksptl I

Teoret Fiz 35, 1358

(1958) V.I.Ostroumov,

Yu.P.Yakovlev

Zhur Ekspu I

Teoret Fiz 35,538

(1958) L.V.Volkova,

E.P.Denisov

Zhur Ekspt I

35,631(1958)

N.A.Perfilov,

G.F.Denisenko

Zhur Eksptl I

Teoret Fiz 35,587

(1958) V.V.Kuznetsov,

V.N.Mekhedov

Zhur Eksptl I

Teoret Fiz 35,56

(1958) B.V.Kurchatov

et al

J Applied Physics

29,698(1958)

W.E.Crandall,

G.P.Millbum

Phys Rev 110,204

(1958) N.Metropolis

et al

Phys Rev 110,185 (1958) N.Metropolis

et al

Phys Rev 109,2086

(1958) Y. Tai et al

Zhur Eksp Teoret

Fiz 33,1185(1957)

L.S.Azhgirei et al fission products, spectra

Monte Carlo method,nuclear cascades,spectra

fission products

particle tracks

recoils

Monte Carlo

method

neutrons

nuclear cascade

emission, knockout reactions 
$12 \mathrm{C}, \mathrm{O}$ Excitation Functions up to $980 \mathrm{MeV}$ for Proton-Induced Reactions in Some Light Elements

U,Th

Th, U

Th, U

U

Th, U

$\mathrm{C}, \mathrm{Pb}$

$\mathrm{Pb}, \mathrm{U}$
MTA Project Target and Process Section Quarterly Progress for September through November 1952

Fission of Heavy Nuclei by High-Energy Particles

Total Neutron Yield from Targets Bombarded by Deuterons and Protons

Neutron Production from HighEnergy Charged Particle Bombardment of Infinite Uranium Targets

Fission and Capt : E Events in 12 Inch X 12 Inch Cross Section Uranium and Thorium Targets Bombarded with $190 \mathrm{MeV}$ Deuterons and $340 \mathrm{MeV}$ Protons

Nuclear Cross Sections for $900 \mathrm{MeV}$ Protons

Measurements of the Numbers of Neutrons Emitted From Thick Targets of Lead and Uriclium When Bombarded with $147 \mathrm{MeV}$ Protons from the 110 Harwell Cyclotron
Proc Phys Soc (London) A70,824 (1957) J.L.Symonds et al

Livermore Research LRL report MTA-30 (1953)

neutron flux

J Nuclear Energy 5,236(1957)

A.K.Lavrukhina, L.D.Krasavina

Berkeley Radiation neutron detection Lab report UCRL. 2063(1957) W.E.Crandall, G.P.Millburn

fission products

Livermore Research

neutron flux

Lab report LRL-69

(1957)C.C.Old

Livermore Research Lab report MTA-6 (1957)H.G.Hicks

Proc Phys Soc (London) A70,209 (1957) N.E.Booth et al

inelastic scattering
Harwell report AERE-N/M-59(1956) no author 
$\begin{array}{ll}\text { O,Sn } & \text { Total Cross Sections for the } \\ U & \text { Interaction of } 650 \mathrm{MeV} \text { Protons } \\ \text { with Nuclei }\end{array}$

$\mathrm{Al}, \mathrm{Be}, \mathrm{Bi} \quad$ Inelastic Scattering of $96-\mathrm{MeV}$

$\mathrm{B}, \mathrm{C}, \mathrm{Cu}, \mathrm{Si} \quad$ Proions

$\mathrm{Pb}$

U

U

U

C

$\mathrm{Al}, \mathrm{Be}, \mathrm{B}$
Radiochemical Studies of the Interaction of Lead with Prowns in the Er.ergy Range 0.6 to $3.0 \mathrm{BeV}$

Fission of Uranium with 170 MeV Protons

Nuclear Reactions of Cobalt with Protons from 0- to 100MeV Energy

Absolute Neutron Spectra from 190-MeV Proton Bombardment of Uranium

Fission of Uranium Nuclei by Protons with an Energy of $460 \mathrm{MeV}$

Cross Sections for Producing High-Energy Neutrons from Carbon Targets Bombarded by Protons, Deuterons, and $3 \mathrm{He}$ Particles

Total Cross Sections of 208$\mathrm{MeV}$ and 315-MeV Protons for Light Elements

Reactions of Cesium with Protons at 60,80, 100, 150, and $240 \mathrm{MeV}$
Doklady Akad. Nauk

SSSR 110,972(1956)

V.I.Moskalev,

B.V.Gavrilovskii

Phys Rev 104, 191

(1956) K.Strauch,

F.Titus

Phys Rev 103,394

(1956) R.Wolfgang

et al

evaporation, spallation

Nucl Phys 1,322 (1956) A.Kjelberg,

\section{A.C.Pappas}

fission products, spallation

Phys Rev 101,1493 (1956) R.A.Sharp

et al

scattering

Berkeley Radiation

Lab report UCRL-3337

spectra

(1956) E.E.Gross

AEC-TR-2307

fission products

Trans. from Doklady

Akad Nauk SSSR 103,

573(1955)

N.S.Ivanov et al

Phys Rev 97,184

(1955) L.Schecter

et al

fast neutrons

Phys Rev 96,398

(1954)

optical model

H.G.De Carvalho

Phys Rev 96,185

(1954) R.W.Fink, E.O.Wiig evaporation, neutrons, pick-up 
$\mathrm{Pb}$

$\mathrm{Ta}, \mathrm{U}$

$\mathrm{V}, \mathrm{Mg}, \mathrm{Co}$

$\mathrm{Li}, \mathrm{O}$

U

Al, Be

$\mathrm{C}, \mathrm{U}$

Be,C

$\mathrm{Cu}, \mathrm{Li}, \mathrm{Pb}$

$\mathrm{Be}, \mathrm{Bi}$

C

$\begin{array}{ll}\text { Al,Be,C } & \text { Nuclear Absorption Cross } \\ \mathrm{Cu}, \mathrm{U} & \text { Sections for High Energy } \\ & \text { Protons }\end{array}$

$\mathrm{Be}, \mathrm{C}$

Co
The Production of Deuterons in High Energy Nucleon Bombardment of Nuclei, and Its Bearing on Nuclear Charge Distribution

Tantalum Spallation and Fission Induced by $340 \mathrm{MeV}$ Protons

Spallation of Vanadium, Manganese, and Cobalt with 187 MeV Protons

Total Cross Sections of 408 $\mathrm{MeV}$ Protons for Hydrogen and Light Elements

Analysis of Secondary Particles Resulting from HighEnergy Nuclear Bombardment

Neutrons Produced by High Energy Protons

On The Interaction of $95-\mathrm{MeV}$ Protons with D, Li, Be, C, $A l, C u$, and $P J$ Nuclei

Neutron Energy Distribution from the Proton Bombardment of $\mathrm{Li}, \mathrm{Be}$, and $\mathrm{C}$ at $375 \mathrm{MeV}$

Nuclear Absorption Cross Protons

Physical Analysis of Be and $C$ Disintegration Induced by $330 \mathrm{MeV}$ Protons

Reactions of $370 \mathrm{MeV}$ Protons with Cobalt
Berkeley Radiation

Lab report UCRL-2670

(1954) Thesis

W.N.Hess

Berkeley Radiation

Lab report UCRL-2542

(1954) Thesis, W.E.Nervik

Phil Mag (7)44,1131

(1953) S.G.Rudstam

Phys Rev 91,767

(1953) J.Marshall

et al

Berkeley Radiation

Lab report

UCRL-2258(1953)

Thesis, R.W.Deutsch

Phil Mag (7)44,425

(1953) T.C.Randle et al

Phys Rev 90,449

(1953) J.A.Hofmann,

K.Strauch

Phys Rev $\underline{89,724}$

(1953) W.F.Goodell

et al

Berkeley Radiation

Lab report UCRL-1967

(1952) Thesis

AJ.Kirschbaum

Berkelley Radiation

Lab neport UCRL-

1914(1952) W.H.Barkas, H.Tynen

Columbia Univ report NYO-3198

(1952) E.Belmont emission

spectra

pick-up

fission products

fission products

evaporation model, particle tracks

fast neutrons

spectra 
$\mathrm{Be}$

$\begin{array}{ll}\begin{array}{l}\mathrm{Be}, \mathrm{C} \\ \mathrm{Pb}\end{array} & \begin{array}{l}\text { Neutron Energy Distribution } \\ \text { from } \mathrm{Be}, \mathrm{C} \text {, and } \mathrm{Pb} \text { Bombarded } \\ \text { by } 245 \mathrm{MeV} \text { Protons }\end{array} \\ & \begin{array}{l}\text { Nuclear Reactions of Iron } \\ \text { with } 340 \mathrm{MeV} \text { Protons }\end{array} \\ \mathrm{C}, \mathrm{W} & \begin{array}{l}\text { Neutron Emission from Nuclei } \\ \text { Excited by High Energy } \\ \text { Protons }\end{array}\end{array}$
$\mathrm{Al}, \mathrm{Cu}$
$\mathrm{Pb}$
12C,Cu High Energy Neutron Yields
$\mathrm{Ag}, \mathrm{U}$
The Range and Straggling of Protons Between 35 and 120 $\mathrm{MeV}$ and Multiple Traversals of a Cyclotron Beam Through Thin Targets

$\mathrm{Al}, \mathrm{C}$

$\mathrm{Be}, \mathrm{C}$

$\mathrm{Al}, \mathrm{Be}, \mathrm{Cr}$

$27 \mathrm{Al}$

C,W
The Angular Distribution of Neutrons Produced in Beryllium by $170 \mathrm{MeV}$ Protons

Elastic Scattering of $340 \mathrm{MeV}$ Protons

Neutron Energy Distribution in Proton Bombardment of $\mathrm{Be}$ and $\mathrm{C}$ at $100 \mathrm{MeV}$

Nuclear Shielding Studies: XI. Fast Neutron Spectra Measured by the Photographic Plate Method(Technical Report No.47)

Nuclear Reactions Resulting from the Proton Bombardment of Numinum

Precision Analysis of Gamma Ray Spectra from High Energy Proton Collisions with Nuclei
Phil Mag (7)43,285

(1952) M.Snowden

fast neutrons

Rochester Univ report NYO-3043

neutron (1952) B.K.Nelson et al

detection

Berkeley Radiation

Lab report UCRL- 1586

(1951) G.Rudstam et al

Phil Mag (7)42,1187

(1951) D.M.Skyrme,

W.S.C.Williams

Phys Rev 83,561

(1951) N.Bloembergen,

P.J.Van Heerden

Berkeley Radiation

Lab report

UCRL-1398(1951)

Thesis, WJ.Knox

Berkeley Radiation

Lab report

UCRL-1284(1951)

R.E.Richardson et al

Phys Rev 82,831

(1951) D.Bodansky,

NF.Ramsey

Mass. Inst. Tech. report NP-1999(1950)

P.H.Stelson

neutron detection proton beams, recoils

spectra

Wisconsin Univ report AECU-1362(19ND)

F.C.Shoemaker et al

Berkeley Radiation Lab report UCRL-1270 (1951) K.M.Crowe et al(Abstract) gamma radiation

gamma radiation 
C. 'b Range Energy Relation for 340-MeV Protons

$\mathrm{Al}, \mathrm{Be}$

C,U

$\mathrm{Be}, \mathrm{Bi}, \mathrm{U}$

$\mathrm{Be}, \mathrm{Bi}$

$\mathrm{Al}, \mathrm{Be}$

$\mathrm{C}, \mathrm{Cu}$

$\mathrm{W}, \mathrm{U}$

$\mathrm{Be}, \mathrm{Bi}$

$\mathrm{C}, \mathrm{U}$

$\mathrm{Be}$
The Production of Neutrons by

High Energy Protons

Relative High Energy Neutron

Yields from Targets Bombarded

with Protons and Deuteroris

Angular Distribution of

Neutrons from the Bombardinent

of Be by $340-\mathrm{MeV}$ Protous

Stopping Power and Energy for Ion Pair Frodiction for 340 -

MeV Protons

Relative High Energy Neutron

Yields from Targets Bombarded with Protons and Deuterons

Production of Very High Energy

Protons by $350 \mathrm{MeV}$ Protons
Berkeley Radiation

Lab report UCRL-1089

(1951) R.Mather,

E.Segre

Phil Mag (7) 42,215

(1951) J.M.Cássels

et al

Berkeley Radiation

Lab report UCRL-440

(Rev)(1950) W.J.Knox

no affiliation

given, AECD-2900

(1950) J.DeJuren

Berkeley Radiation

Lab report UCRL-850

(1950) C.J.Bakker,

E.Segre

no affiliation

given(Berkeley?)

AECD-2837(1949)

W.J.Knox

no affiliation

given AECU-280(19ND)

H.F.York et al slowidown

neutron flux, spectra

fast neutrons

fast neutrons

slowdown

stripping

gamma radiation 
7. H. J. Kim, et al., Nuclear Cross Sections for Charged-Particle-Induced Reactions. Nucl. Data A1, 203 (Academic Press, New York, 1965). See also Nuc1. Data A2, 1 (Acudemic Press, New York, 1966) and Nucl. Data A3, 123 (Academic Press, New York, 1967).

8. H. Münzel, H. Klewe-Nebenius, J. Lange, G. Pfennig, K. Hemberle, "Karlsruhe Charged Particle Reaction Data Compilation," Karlsruhe reports Physik Dater 15-Index (1979), Physik Daten 15-1 (1979), Physit Daten 15-2 (1979), Physik Daten 15-3 (1982), Physik Daten 15-4 (1982), and Physik Daten 15-5 (198:).

9. F. K. McGowan, W. T. Milner, H. J, Kim, "Nuclear Cross Sections for Charged-Particle Induced Redctions - Mn, Fe, Co," Oak Ridge National Laboratory report ORNL-CPX-1 (1964); and "Nuclear Cross Sections for ChargedParticle Induced Reactions $\mathrm{Ni}, \mathrm{Cu}, "$ Oak Ridge National Laboratory report ORNL-CPX-2 (1964).

\section{ACKNOWLEDGMENTS}

Special thanks are given to $M$. Treiman and $C$. Nielsen ot the Los Alamos National Laboratory Main Library for their invaluable assistance and patience in performing the RECON search upon which this report is based. 


\section{DEUTERON INDUCED REACTIONS}

TARGET

TIILE

${ }^{12} \mathrm{C}, 197 \mathrm{Au}$

$208 \mathrm{~Pb}$

Fragmentation Processes

In Nuclear Reactions

Nucleon and Deuteron

Scattering Cross Sections

from $25 \mathrm{MeV} /$ Nucleon to

$22.5 \mathrm{GeV} /$ Nucleon

$232 \mathrm{Th}$

$55 \mathrm{Mn}$

$27 \mathrm{Al}, 11^{\circ} \cdot \mathrm{Au}$

$181 \mathrm{Ta}$

160

$93 \mathrm{Nb}$

${ }^{9} \mathrm{Be}, 6 \mathrm{Li}$

160

$93 \mathrm{Nb}$
Coulomb-Dissociation at

Relativistic and Non-

Relativistic Energies

Mediun-Energy Reaction

Cross Sections for Light

Composite Projectiles

(d,nn) Reaction on Nuclei in the Medium Energy Range

Projectile Fragmentation and Stripping to Unbound States: An Important Reaction Mechanism for Peripheral NucleusNucleus Collisions

Comment on (d.p) Stripping Model at $700 \mathrm{MeV}$

Continuum of tt. Spectra of Emirted Charged

Particles

Investigation of the

(d.p) Stripping Reaction

Around $700 \mathrm{MeV}$

Importance of the Breakup Mechanism for Composite Particle Scattering
REFERENCE

DESCRIPTORS

Darmistadt report

quasi-free reactions

GSI-83-82 p.79,

(1983) (Preprint)

G.Baur et al

NASA report, NASA.

particle

TM-84636(1983)

L.W. Wownsend, J.W.Wilson

interactions

Julich report

JUEL-SPEZ-146, p.95

(1982) G.Baur,

B.Hoffmann

$\mathrm{J}$ of Physics u8, 39(1982) H.Nishicka,

R.C.Johnson

J Ukr Phys 26,86

(1981) V.V.Piliperiso

Helv Phy Acta

33,506 (1981)

G.Baur et al

inelastic

scattering

lotal cross sections

diffrraction models

optical models,

stripping

Phys Rev Lett 46 ,

1544(1981) J R.Shepard,

E.Rost

Gross Properties of

Nuclei and Nuclear

Excitations. Proc. Julich

Pt. A: Nuclear

Collisions, INKA-CONF-

79-001-000 p.82 (1979)

H.Machner

Fhys Rev Lett 46 , 218(1981) A.Boudard

et al

one-nucleon

trarisfer reactions

Phys Rev C21,2658

(1930) G.Baur et al one-nucleon

transfer reactions optical models 
$9 \mathrm{Be}, 93 \mathrm{Nb}$

$120 \mathrm{Sn}, 27 \mathrm{Al}$

Li, 232Th

V

${ }^{59} \mathrm{Co}$
Continuous Particle Spectra

from Direct Break-up

Reactions

Deuteron and $3 \mathrm{He}$ Scattering:

Discussion on the Uniqueness of Their Optical

Potentials

Intermediate Energy

Deuteron-Nucleus Diffrac-

tion Interaction

Electronuclear Conversion of Fertile to Fissile

Material

Global Viewpoint on

Nuclear-Particle-Emission

Cross Sections at Inter-

mediate Energies

Excitation Functions of

Deuteron Induced Nuclear

Reactions on Vanadium with

Special Reference to the

Production of $43 \mathrm{~K}$ : Systematics

of $(d, x n)$ Reaction Cross

Sections Revelant to the

Formation of Highly Neutron

Deficient Radioisotopes

Mechanism of Break-Up

Reactions of Light Ions at

Non-Relativistic Energies

Measurement and Hybrid Model

Analysis of Integral Excitation

Functions for Light Particle

Induced Reactions
8. Int Symp on the

Interaction of Fast

Neutrons with Nuclei,

Gaussig, German Demo-

cratic Republic ZFK-382

p.99(1978) G.Baur et al

Workshop on Microscopic Optical Potentials Hamburg, F.R.Germany, CONF-780971(1978)

\section{A.Djaloeis}

Nucl Phys A308, 330(1978)

A. Yu.Berezhnoy, A.P.Soznik

Lawrence Livermore Nat Lab repori UCRL52144(1976)

C.M.Van Atta et al

Phys Rev Lett 36,

1464(1976)

Y.Iwasaki

Radiochim Acta 35,

11(1984)

S.M.Qaim, ت्ञ.J.Probst breakup reactions, direct reactions

optical models

breakup reactions, stripping

neutrons sources, nuclear cascades

pickup reactions

excitation

functions
INIS-MF-9280(1984)

Workshop on

Coincident Particle

Emission from Con-

tinuum States, Bad

Honnef,F.R.Ger, Jun 4

1984, G.Baur et al

Julich report

JUEL-SPEZ-255,p.32

(1984) R.Michel et al knock-out

reactions 
$\begin{array}{ll}12 \mathrm{C}, 118 \mathrm{Sn} & \text { Coupled-Channel Approach to } \\ \text { S1V } & \text { Deuteron Projectile Breakup }\end{array}$

$208 \mathrm{~Pb}$

Deuteron Breakup in the Field of a Heavy Target

Co

$12 \mathrm{C}$

$118 \mathrm{Sn}$

$27 \mathrm{Al}$

$209_{\mathrm{Bi}}$

$62 \mathrm{Ni}$

$93 \mathrm{Nb}$

$58 \mathrm{Ni}$

$197 \mathrm{Au}$

$208 \mathrm{~Pb}$

$181 \mathrm{Ta}$

$58 \mathrm{Ni}$

Investigation of Inclusive

Spectra of Nuclear Reaction

Products with Participation

of Deuterons and ${ }^{3} \mathrm{He}$ Nuclei

at Intermediate Energies

Caliulation of Proton-Neutron

Coincidence Cross Sections in

Deuteron Break-Up at $56 \mathrm{MeV}$

New Formalism of Inclusive

of the Surface Approximation

Nucleus Collisions

Total Cross Sections of Inel-

astic Processes of Deuteron
Phys Lett B141,19

(1984) M.Yahiro

et al

Phys Rev C2y, 1095

(1984)

S.N.Mukherjee et al

Int J Appl Rad \&

Iso $34,1325(1983)$

R.Michel, M.Galas

$56 \mathrm{MeV}$ Deuteron-Induced Break-

up Reactions by Post Form Dis-

torted-Wave Born Approximation

Proton Continuum Spectra from

Breakup Reactions and Validity

Study of Various Discretization

Methods of the Neutron-Proton

Breakup Continuum in Deuteron

Interactions with Heavy Nuclei

Inelastic Scattering and

Inelastic Breakup of

Deuterons by Nuclei
INIS-SU-198 p.441

23. Conf Nucl Spect

\& Nucl Structure

Apr 19,1983,Moscow,

M.V.Evlanov et al

Phys Rev C28,946

(1983) G.Baur

et al

Int Symp on Highly

Excited States in

Nuclear Reactions

Osaka,Japan May 12,

1980, p.88, N.Matsuoka

et al

Phys Lett $\underline{115,81}$

(1982) A.Kasano

et al

U. of Conn.,Thesis

(1982)R.Y.Rasoanaivo

Izv Akad Nauk Kaz

4,20(1981)

M.G.Itkis et al

Phys Rev C26,904

(1982) H.Amakawa,

T.Tamura

breakup reactions, breakup reactions

quasi-elastic

scattering

breakup reactions

stripping,

transfer reactions stripping, inclusive interactions

breakup reactions

breakup reactions

inclusive interactions

inelastic scattering

breakup reactions 


\begin{tabular}{|c|c|}
\hline $\begin{array}{l}52 \mathrm{Cr}, \mathrm{V} \\
6 \mathrm{Li}, \mathrm{Sc}\end{array}$ & $\begin{array}{l}\text { Total Cross Sections for } \\
\text { Interaction of Deuterons and } \\
\text { Alpha Particles with Nuclei } \\
\text { (Approx.S0) }\end{array}$ \\
\hline${ }^{59} \mathrm{Co}$ & $\begin{array}{l}\text { Measurement and Hybrid Model } \\
\text { Analysis of Integral Excita- } \\
\text { tion Functions of } 2 \mathrm{H} \text {-Induced } \\
\text { Reactions on Cobalt }\end{array}$ \\
\hline $52,53,54 \mathrm{Cr}$ & $\begin{array}{l}\text { Deuteron and Alpha-Particle } \\
\text { Total Reaction Cross Sections } \\
\text { for Nuclei with A Approximate- } \\
\text { ly } 50\end{array}$ \\
\hline $233 \mathrm{U}$ & $\begin{array}{l}\text { Deuteron-Induced Fission in } \\
233 \mathrm{U}\end{array}$ \\
\hline $235 \mathrm{U}$ & $\begin{array}{l}\text { Lifetimes of the Nuclei Formed } \\
\text { by Deuteron Bombardment of } \\
235 \mathrm{U}\end{array}$ \\
\hline $207 \mathrm{~Pb}$ & $\begin{array}{l}\text { Preliminary Computer Process- } \\
\text { ing of Neutrc : Spectra } \\
\text { Obtained in the Deuteron } \\
\text { Spallation Reaction }\end{array}$ \\
\hline
\end{tabular}

$\mathrm{Pb}$

$27 \mathrm{Al}$

${ }^{209} \mathrm{Bi}$

$12 \mathrm{C}$

${ }^{232} \mathrm{Th}$ Inelastic Break-Up Phenomena in $\mathrm{d}-$ and $6 \mathrm{Li}$-Induced Reactions on $232 \mathrm{Th}$

Cross Sections and Thick Target Yields of $(d, x n)$ Reactions on Natural Lead

Deuteron Break-Up in the Fields of Nuclei at $56 \mathrm{MeV}$

$197 \mathrm{Au}$
Sov J Nucl Phys

35,317(1982)

O.P.Bilanyuk

et al

Verhandlungen der

Deutschen Physi-

kalishen Gesell-

schaft 17, 1270(1982)

(E.Ger) M.Galas et al

J of Physics G7. 1699(1981)

O.M.P.Bilaniuk

et al

Can J Physics 59 , 255(1981) N.Mobed et al

Nucl Instr Meth $170,145(1980)$

N.G.Chechenin et al

27.All-Union Conf on Nucl Spect. and

Nucl Struct. Mar 22, 1977 Tashkent, USSR, p.321,V.T.Grantsev et al

Radiochimica Acta 27,125(W.Ger)(1980)

C.Wasilevsky et al

Nucl Phys A345, 1 (1980) N.Matsuoka et al

Verhandlungen der Deutschen Physikalischen Gesellschaft 16, 792(1981) J.Kleinfeller et al

Selected Topics in Nucl Struct, 18th Winter School, Bielsko-Biala,Poland 1980, INP-1134/PL, p.107, H.Machner total cross sections, optical model

integral cross sections

optical models

fission

fission products

neutron spectra, spallation

transfer reactions

breakup reactions, stripping

breakup reactions

breakup reactions, precompound nucleus emission 
Table II (Cont.)

$27 \mathrm{Al}$

197 Au

160

$238 \mathrm{U}$

$\mathrm{Li}$

$52 \mathrm{Cr}$

$56 \mathrm{Fe}$

$58 \mathrm{Ni}$

${ }^{27} \mathrm{Al}$

$58 \mathrm{Ni}$

$\mathrm{Li}$

${ }^{63} \mathrm{Cu}$

$27 \mathrm{Al}$
Experimental and Theoretical

Study of Continuous Proton

Spectra from High-Energy

Deuteron Induced Reactions

New Method to Describe the

Stripping to Unbound States

and Other Reactions with

Unstable Particles

Global Optical Model Analysis

of the Elastic Deuteron Scat-

tering from Medium Heavy

Target Nuclei

238U(d,2n) 238 Np and

238U(d,p,d,n) 239 Np Reactions

at Moderate Excitation

Energies

Measurement and Calculations

of Neutron Spectra from $35 \mathrm{MeV}$

Deuterons on Thick Lithium

for the FMIT Facility

Global Optical-Model Analysis

of the Elastic Deuteron Scat.

tering from Medium Heavy

Target Nuclei

Multiparticle Removal Reactions Induced by Deuterons and the Study of Pre-Equilibrium Reactions

High-Flux Neutron Generator with a Linear Accelerator

Composite Particle Emission from $65 \mathrm{Zn}$

Global Optical Model Potential

for Elastic Deuteron Scatter. ing
Nucl Phys A34j, ?21

(1980) U.Bechstedt

et al

Phys Lett B96,4

(1980)

V.M.Krasnopol'skii,

V.I.Kukulin

Aust Nat Univ (Canberra) report ANU-

P-753(1979)

H.R.Burgi et al

Arab J of Nucl Sci

and Appl.(Egypt)

12,83(1979)

A.T.Kandil

J Nucl Materials

$85,467(1979)$

D.L.Johnson et al

Nucl Phys A334,413

(1980) H.R.Buergi

et al

U of Maryland

Thesis(1977)

J.R.Wu

All Union Conf on

Charged-Particle

Accelerators, Dubna,

JîR-D 0-1 1874,p.33

(1978) A.A.Vasil'ev et al

Phys Rev C21,2695

(1980) H.Machner

Phys Rev C21,2253

(1980)

W.W. Daehnick et al stripping

elastic

scattering

breakup reactions

optical models

excitation

functions

neutron spectra

precompound-

nucleus emission

stripping

precompound-

nucleus emission

elastic

scattering, optical mndels 
Be

$197 \mathrm{Au}$

$207 \mathrm{~Pb}$

$208 \mathrm{~Pb}$

27 Al

${ }^{197} \mathrm{Au}$

$\mathrm{Be}$

$197 \mathrm{Au}$

$58 \mathrm{Ni}$

$\mathrm{Be}, \mathrm{C}, \mathrm{Al}$

$\mathrm{Ni}, \mathrm{Cu}, \mathrm{Mo}$

$\mathrm{Ta}, \mathrm{Pb}$

$197 \mathrm{Au}$

$208 \mathrm{~Pb}$

$\mathrm{Li}$

$9 \mathrm{Be}$
Monte Carlo Calculation of d-Be Neutron Yields

Neutron-Nucleus Interaction and Some Features of Deuteron

Disintegration by Heavy Nuclei

Continuous Charged Particle

Spectra from Deuteron Induced

Reactions

Intense Neutron Source Based

on $79 \mathrm{MeV}$ Deuterons Bombarding

Beryllium

Charged-Particle Spectra: 80

$\mathrm{MeV}$ Deuterons on $27 \mathrm{Al}$ and $58 \mathrm{Ni}$ and $70 \mathrm{MeV}$ Deuterons on $90 \mathrm{Zr}$, $208 \mathrm{~Pb}$, and $232 \mathrm{Th}$

Diffraction Spallation of

Deuterons by Nuclei

Disintegration of Medium-

Energy Deuterons by Atomic

Nuclei

Energy Spectra of Neutrons

from Thick Targets Bombarded

by Deuterons

Deuteron Disintegration on

Heavy Nuclei and Allowance

for Neutron Interaction in

a Final State

Intense Neutron Source Based

upon the Deuteron-Stripping

Reaction

Generation of Fast Neutrons in a Cyclotron Using Helium-3 Ions
Nucl Instr Meth

160,499(1979)

D.Christenson et al

Bull Acad Sci USSR

Phys Series

41,1683(1977)

V.I.,Grantsev et al

Julich report JUEL-

SPEZ-15 p. 21(1978)

U.Bechstedt et al

Nucl Instr Meth

154,399(1978)

G.H.Harrison,

E.B.Kubiczek

Phys Rev C19,370

(1979) J.R.Wu

et al

Bull Acad Sci USSR

Phys Series

41,1274(1977)

M.V.Evlanov et al

Ukr Phys J 21,1174

(1976)

Yu.A.Berezhnoi et al

Sov At Energy 43,

209(1977)

V.P.Badovstii et al

Ukr Phys J 22,238

(1977) V.I.Grantsev

et al

Nucl Instr Meth

145,49(1977)

P.Grand, A.N.Goland

Int J Appl Rad \&

Isotopes 28,459(1977)

B. $V$ Zatolokin et al stripping

spallation

proton spectra

fast neutrons

precompoundnucleus emission

breakup reactions, spallation

breakup reactions, spallation

neutron spectra

breakup reactions

neutron spectra

neutron spectra, fast neutrons 
232Th Mechanism of the Reaction 232Th(d,2n)232 $\mathrm{Pa}$

${ }^{9} \mathrm{Be}$

$92,94 \mathrm{Mo}$

$98,100 \mathrm{Mo}$

$238 \mathrm{U}$

${ }^{9} \mathrm{Be}$

${ }^{27} \mathrm{Al}$

$\mathrm{Be}$

$12 \mathrm{C}$

${ }^{27} \mathrm{Al}, 63 \mathrm{Cu}$

$197 \mathrm{Au}$

$76 \mathrm{Ge}$

$64 \mathrm{Zn}$

$\mathrm{Pt}$

${ }^{9} \mathrm{Be}$
Stripping-Theory Analysis of

Thick-Target Neutron Produc-

tion for $d+B e$

Excitation Functions and

Yields of $(d, n)$ and $(d, 2 n)$

Reactions on Natural

Molybdenum

Deuteron Induced Fission of 238U

Neutron Spectrum at 00 from 533.7 MeV Deuterons on

Beryllium

Study of Proposed ${ }^{27} \mathrm{Al}$ Models by an Analysis of Inelastic Scattering Data of 60 to 90 MeV Deuterons

Fast-Neutron Dose Rate vs Energy for the $d+$ Be Reaction:

A Reanalysis

Elastic and Inelastic Deuteron Scattering on $12 \mathrm{C}$ in the Energy Range from 60 to $90 \mathrm{MeV}$

Highly Inelastic Scattering of Medium Energy Deuterons

Excitation Functions for the

Deuteron Induced Reactions on $64 \mathrm{Zn}$ and $76 \mathrm{Ge}$

Low-Temperature Energy Release in Platinum after Ion Radiation

Intensity Measurements and Shielding of a Fast-Neutron Beam for Biological and Medical Application

OER of Neutrons from $80 \mathrm{MeV}$ Deuterons on Beryllium.
Phys Rev C15,2255 (1977) J.Rama Rao et al

precompoundnucleus emission

Phys in Med \& Bio

neutron spectra

21,931(1976)

L.S.August et al

J Inorg \& Nucl

Chem 38,2289(1976)

Z.Randa, K.Svoboda

stripping,

gamma spectra

J Inorg \& Nucl

Chem 38,975(1976)

\section{A.T.Kandil}

Phys Rev C14,801

(1976) R.Madey

et al

Phys Rev C13,1400 (1976) K.T.Knopfle

et al

Med Phys 3,45(1976)

J.B.Smathers et al

fast neutrons

Nucl Phys A 253,

263(1975)

O.Aspelund et al

U of Wash. Seattle

Wa., Thesis(1975)

H.H.Wieman

elastic

scattering

Bull Inst Chem Res, Kyoto Univ, 52,233 (1974) T.Nishi et al

Phys Rev B12,1217 (1975) J.J.Jackson

physical radiation effects

Nucl Instr Meth

neutron spectra

126,81(1975)

J.P.Meulders et al

Br J Radiol 48,569

neutron beams (19i4) J.L.Fowler

et al 
Table II (Cont.)

\begin{tabular}{|c|c|c|c|}
\hline${ }^{9} \mathrm{Be}$ & $\begin{array}{l}\text { Neutron Spectra at } 00 \text { from } \\
\text { 83.7-MeV Deuteron Bombardment } \\
\text { of a Thick Beryllium Target }\end{array}$ & $\begin{array}{l}\text { Kent State Univ } \\
\text { report COO-2231-27 } \\
\text { (1975) R.Madey et al }\end{array}$ & $\begin{array}{l}\text { neutron spectra, } \\
\text { stripping }\end{array}$ \\
\hline $12 \mathrm{C}$ & $\begin{array}{l}\text { Neutron Spectra at } 00 \text { from } \\
74-\mathrm{MeV} \text { Deuteron Bombardment } \\
\text { of a Thick Carbon Target }\end{array}$ & $\begin{array}{l}\text { Kent State Univ } \\
\text { report COO-2231-24 } \\
\text { (1974) R.Madey et al }\end{array}$ & neutron spectra \\
\hline $\begin{array}{l}9 \mathrm{Be} \\
12 \mathrm{C}\end{array}$ & $\begin{array}{l}\text { Fast Neutron Yields and } \\
\text { Spectra from Targets of Vary- } \\
\text { ing Alomic Number Bombarded } \\
\text { with Deuterons from } 16 \\
\text { to } 50 \mathrm{MeV}\end{array}$ & $\begin{array}{l}\text { Phys Med Biol 20 } \\
\text { 235(1975) } \\
\text { J.P.Meulders }\end{array}$ & $\begin{array}{l}\text { gamma spectra, } \\
\text { neutron spectra }\end{array}$ \\
\hline $40 \mathrm{Ca}$ & $\begin{array}{l}\text { Johnson and Soper's Method of } \\
\text { Including Deuteron Break-Up } \\
\text { for the Calculacion of } \\
\text { Stripping Cross Sections }\end{array}$ & $\begin{array}{l}\text { Nucl Phys A241,365 } \\
\text { (1975) } \\
\text { G.H.Rawitscher }\end{array}$ & stripping \\
\hline $\begin{array}{l}27 \mathrm{Al}_{,}^{63} \mathrm{Cu} \\
208 \mathrm{~Pb} \\
181 \mathrm{Ta}\end{array}$ & $\begin{array}{l}\text { Highly Inelastic Deuteron } \\
\text { Scattering at } 45 \mathrm{MeV}\end{array}$ & $\begin{array}{l}\text { Univ Washington } \\
\text { report RLO-1388- } \\
258 \text { p.65(1974) } \\
\text { I.Halpern et al }\end{array}$ & $\begin{array}{l}\text { inelastic } \\
\text { scattering }\end{array}$ \\
\hline \multirow[t]{2}{*}{$\begin{array}{l}\mathrm{Ca}, \mathrm{Cl} \\
\mathrm{Cu}\end{array}$} & $\begin{array}{l}\text { Binding Effects in High-Energy } \\
\text { Scattering Applied to K-Shell } \\
\text { Ionization }\end{array}$ & $\begin{array}{l}\text { Phys Rev Al1, } 1205 \\
\text { (1975) J.Binstock, } \\
\text { J.F.Reading }\end{array}$ & ionization \\
\hline & $\begin{array}{l}\text { Continuous Proton Spectra } \\
\text { Emitted in } 3 \mathrm{He} \text { and Detiteron } \\
\text { Induced Reactions }\end{array}$ & $\begin{array}{l}\text { Nuci Phys A237,354 } \\
\text { (1975) A.Chevarier } \\
\text { et al }\end{array}$ & $\begin{array}{l}\text { breakup reactions, } \\
\text { stripping }\end{array}$ \\
\hline \multirow[t]{4}{*}{$181 \mathrm{Ta}$} & $\begin{array}{l}\text { Analysis of (particle,xn) } \\
\text { Reactions on Tantalum and } \\
\text { Gold. III. Excitation Func- } \\
\text { tions of d-Induced Reactions } \\
\text { on } 181 \text { Ta from } 10 \text { to } 80 \mathrm{MeV} \\
\text { and Equilibrium Statistical } \\
\text { Analysis }\end{array}$ & $\begin{array}{l}\text { Nucl Phys A228, } 180 \\
\text { (1974) } \\
\text { J.Bisplinghoff } \\
\text { et al }\end{array}$ & Monte Carlo method \\
\hline & $\begin{array}{l}\text { Color Centers Due to Cationic } \\
\text { Defects Created by High Energy } \\
\text { lons in LIF and KCL }\end{array}$ & $\begin{array}{l}\text { J Phys (Paris) } \\
\text { Colloq No. } 9.289 \\
\text { (1973) P.Thevenard } \\
\text { et al }\end{array}$ & $\begin{array}{l}\text { physical radiation } \\
\text { effects, } \\
\text { recombination }\end{array}$ \\
\hline & $\begin{array}{l}\text { Production of } 14-\mathrm{MeV} \text { Neutrons } \\
\text { by Heavy Ions }\end{array}$ & $\begin{array}{l}\text { Aeroject Nucl Co. } \\
\text { report ANCR-1154 } \\
\text { (1974) R.M.Brugger } \\
\text { et al }\end{array}$ & $\begin{array}{l}\text { heavy reactions, } \\
\text { neutron sources, } \\
\text { recoils }\end{array}$ \\
\hline & $\begin{array}{l}\text { Transfer Reactions Induced by } \\
\text { Lithium Ions }\end{array}$ & $\begin{array}{l}\text { Sov J Particles } \\
\text { Nucl 3,467(1973) } \\
\text { A.A.Ogloblin }\end{array}$ & $\begin{array}{l}\text { dispersion } \\
\text { relations, } \\
\text { excitation } \\
\text { functions }\end{array}$ \\
\hline
\end{tabular}


6Li Medium Enengy Cluster KnockOut Reactions on $6 \mathrm{Li}$ and Their 'bs:spretation in Terms of Distorted Waves

${ }^{9} \mathrm{Be}$

$$
\text { Be,C }
$$$$
\mathrm{Li}
$$

$24 \mathrm{Mg}$

$89 \mathrm{Y}$

Au

$40 \mathrm{Ca}$

$12 \mathrm{C}$

6Li Quasi-Free Scattering in the

Cs Polarization of Fluorescent

197 Au Deuteron Break-Up in the

${ }^{12 \mathrm{C}, 9 \mathrm{Be}} \quad$ Spectra of Neutrons from $10 \mathrm{~B}, 7 \mathrm{Li}$

$27 \mathrm{Al}$

${ }^{9} \mathrm{Be}$

$181 \mathrm{Ta}$

28Si,C

$\mathrm{O}, \mathrm{Mg}$ $6 \mathrm{Li}(\mathrm{d}, \mathrm{d}$ alpha) $2 \mathrm{H}$ Reaction at $52 \mathrm{MeV}$ Light Emitted by Plastic Ne102A, Ki(Tl), and Anthracene Field of the Nucleus

Fast Neutron Dose Rate as a Function of Incident Deuteron Energy for $d+{ }^{9} \mathrm{Be}$

Neutron Spectra from Deuteron Bombardment of D, Li, Be, and C

Optical Model and CoupledChannel Analysis of $60-90 \mathrm{MeV}$ Deuteron Scattering from $24 \mathrm{Mg}$ and $89 Y$

Measurement and Interpretation of 197Au(d,xnyp) Excitation Functions in the Energy Range from 25 to $86 \mathrm{MeV}$

Deuteron-Nucleus Reactions at 69.1 MeV and a Determination of Neutron Detector Efficiencies

Deuteron Bombardment of Light Nuclei

Spectra and Absolute Yields of Neutrons from Thick Targets Bombarded by $23.2 \mathrm{MeV}$ Deuteron

Multistep Processes in the (d, 6Li) Reaction
Nucl Phys A216,519

(1973) A.KJain

et al

impulse approximation, knock-out reactions

Phys Med Biol 18 , 641(1973)

L.S.August et al

Nucl Sci Eng \$2,35

(1973) K.A.Weaver

et al

Zeit fur Phys

260, 179(1973)

G.Hrehuss et al

Nucl Phys A209,333

(1973) PJahn

et al

$\mathrm{U}$ of Maryland

Thesis(1972)

G.H.Harrison

Nucl Phys A207,366

(1973) R.Hagelberg et al

Zeit fur Phys 258 , 251(1973) AE.Wohanka

Nucl Phys A204,97

(1973) J.Lang et al

Lawrence Livermore

Nat Lab report UCRL-

51310(Add.)(1973)

K.A.Weaver

At Energ (USSR)

33,934(19.72)

V.X.Daruga et al

Phys Lett B40,456

(1972) J.R.Comfort fast neutrons

neutron spectra

elastic scattering, optical models

hybrid models, excitation functions

inelastic scattering, pickup reactions, stripping

cluster model

fluorescence

stripping

neutron spectra

neutron spectra

pickup reactions 


\begin{tabular}{|c|c|c|c|}
\hline $\begin{array}{l}\mathrm{Li}, \mathrm{Be} \\
\mathrm{C}, \mathrm{Al}\end{array}$ & $\begin{array}{l}(d, n) \text { Thick-Target Yields and } \\
\text { Total Cross Sections Between } \\
1 \text { and } 40 \mathrm{MeV}\end{array}$ & $\begin{array}{l}\text { J Appl Phys 43, } \\
\text { 3886-8(1972) } \\
\text { L.L.Lucas, J.W.Root }\end{array}$ & $\begin{array}{l}\text { thick-target } \\
\text { neutron yield }\end{array}$ \\
\hline As & $\begin{array}{l}\text { Excitation Functions for } \\
\text { Deuteron Reactions with } 75 \text { As }\end{array}$ & $\begin{array}{l}\text { J Inorg Nucl Chem } \\
\text { 34,1773(1972) } \\
\text { H.F.Roehm, H.Muenzel }\end{array}$ & $\begin{array}{l}(d, p),(d, p \times n) \text {, } \\
\text { and }(d, x n)\end{array}$ \\
\hline $\begin{array}{l}\mathrm{Cl}, \mathrm{Cu} \\
\mathrm{Ali}, \mathrm{Ni} \\
\mathrm{Sn}, \mathrm{Te}\end{array}$ & $\begin{array}{l}\text { Projectile Z Dependence of } \mathrm{K} \\
\mathrm{X} \text {-Ray Emission Induced by } \\
\text { Alpha Particles and Deuterons }\end{array}$ & $\begin{array}{l}\text { Phys Rev A5, } 1773 \\
\text { (1972) C.W.Lewis et al }\end{array}$ & x-radiation \\
\hline $\mathrm{Au}$ & $\begin{array}{l}\text { Reactions Induced in Au by } 10 \\
\text { to } 70 \mathrm{MeV} \text { Deuterons }\end{array}$ & $\begin{array}{l}\text { J Phys (Paris) } 32 \\
483(1971) \\
\text { N.Chevarier et al }\end{array}$ & transfer reactions \\
\hline $\begin{array}{l}27 \mathrm{AJ}, 12 \mathrm{C} \\
208 \mathrm{~Pb}, 68 \mathrm{Zn} \\
120 \mathrm{Sn}, 89 \mathrm{Y}\end{array}$ & $\begin{array}{l}\text { Elastic and Inelastic Scat- } \\
\text { tering of } 80-\mathrm{MeV} \text { Deuterons }\end{array}$ & $\begin{array}{l}\text { Nucl Phys A174,485 } \\
\text { (1971) G.Duhamel } \\
\text { et al }\end{array}$ & $\begin{array}{l}\text { elastic } \\
\text { scattering, } \\
\text { optical model }\end{array}$ \\
\hline${ }^{197} \mathrm{Au}$ & $\begin{array}{l}\text { Reactions Induced in Gold by } \\
\text { Deuterons with Energies } \\
\text { between } 10 \text { and } 70 \mathrm{MeV}\end{array}$ & $\begin{array}{l}\text { Lyon Univ (France) } \\
\text { report LYCEN-7092 } \\
\text { (1970) N.Chevarier et al }\end{array}$ & $\begin{array}{l}\text { excitation } \\
\text { functions }\end{array}$ \\
\hline $9 \mathrm{Be}$ & $\begin{array}{l}\text { Production of Neutrons Through } \\
\text { Irradiation of Thick Beryllium } \\
\text { Targets with Deuterons in the } \\
\text { Energy Range from } 15 \text { to } 55 \mathrm{MeV}\end{array}$ & $\begin{array}{l}\text { Karlsnuhe rport } \\
\text { KFK-1288(1970) } \\
\text { K.Schmidt, H.Muenzel }\end{array}$ & neutron spectra \\
\hline $59 \mathrm{Co}$ & $\begin{array}{l}\text { Measurement of the Excitation } \\
\text { Functions of }(d, x) \text { Reactions } \\
\text { on } 59 \mathrm{Co}\end{array}$ & $\begin{array}{l}\text { Radiochim Acta 15, } \\
\text { 13(1971) R.Bilabel }\end{array}$ & $\begin{array}{l}\text { excitation } \\
\text { functions }\end{array}$ \\
\hline $\begin{array}{l}\mathrm{Cu}, \mathrm{Au} \\
\mathrm{Ti}\end{array}$ & $\begin{array}{l}\text { Precise Test of the Z2 } \\
\text { Dependence of X-Ray Emission } \\
\text { Induced by Alpha } \\
\text { Particles and Deuterons }\end{array}$ & $\begin{array}{l}\text { Phys Rev Lett } 26 \\
481(1971) \text { C.W.Lewis } \\
\text { et al }\end{array}$ & $x$-radiation \\
\hline $\mathrm{Cu}$ & $\begin{array}{l}\text { Excitation Functions for } \\
\text { Radioactive Nuclides } \\
\text { Produced by Deuteron-Induced } \\
\text { Reactions in Copper }\end{array}$ & $\begin{array}{l}\text { Nucl Phys A155,40 } \\
\text { (1970) C.B.Fuimer, } \\
\text { I.R.Williams }\end{array}$ & $\begin{array}{l}\text { excitation } \\
\text { functions }\end{array}$ \\
\hline 197 Au & $\begin{array}{l}\text { Inelastic Scattering of } \\
\text { Deuterons from } 197 \mathrm{Au}\end{array}$ & $\begin{array}{l}\text { Nucl Phys A154, } 312 \\
\text { (1970) R.P.Sharma }\end{array}$ & $\begin{array}{l}\text { inelastic } \\
\text { scattering }\end{array}$ \\
\hline $\mathbf{S i}$ & $\begin{array}{l}\text { Energy Spectra of Radiation } \\
\text { Defects Generated in Si by } \\
\text { Deuteron Irradiation }\end{array}$ & $\begin{array}{l}\text { Sov Phys Semicond } \\
\text { 3,132(1969) } \\
\text { I.P.Akimchenko et al }\end{array}$ & photoconductivity \\
\hline
\end{tabular}




\begin{tabular}{|c|c|}
\hline $\begin{array}{l}209 \mathrm{Bi} \\
197 \mathrm{Au} \\
235,238 \mathrm{U}\end{array}$ & $\begin{array}{l}\text { Fragment Angular Distributions } \\
\text { in the Fission of Heavy Nuclei } \\
\text { by } 103 \mathrm{MeV} \text { Helium Ions and } \\
51.5 \mathrm{MeV} \text { Deuterons }\end{array}$ \\
\hline $\mathrm{Ni}$ & $\begin{array}{l}\text { Scattering of Fast Deuterons } \\
\text { at Large Angles }\end{array}$ \\
\hline $197 \mathrm{Au}$ & $\begin{array}{l}\text { Reactions Induced in Gold by } \\
\text { Deuterons of Energies up to } \\
60 \mathrm{MeV}\end{array}$ \\
\hline $\mathrm{U}$ & $\begin{array}{l}\text { Interaction of High-Energy } \\
\text { Deuteron Beams with Matter }\end{array}$ \\
\hline $\mathrm{U}$ & $\begin{array}{l}\text { Interaction of High-Energy } \\
\text { Deuteron Beam with Matter }\end{array}$ \\
\hline $\mathrm{Be}$ & $\begin{array}{l}\text { Time-of-Flight Spectrometer } \\
\text { for Neutrons from } 1 \text { to } 500 \mathrm{MeV}\end{array}$ \\
\hline \multirow[t]{2}{*}{$\begin{array}{l}\mathrm{Cu}, \mathrm{Pb}, \mathrm{C} \\
\mathrm{Cd}, \mathrm{Al}\end{array}$} & $\begin{array}{l}\text { Deuteron Stripping at } 1.69 \\
\text { GeV/c. II. Neutron Distribu- } \\
\text { tions }\end{array}$ \\
\hline & $\begin{array}{l}\text { Certain Characteristics of } \\
\text { Fast Deuteron Interactions } \\
\text { with Nuclei }\end{array}$ \\
\hline $\begin{array}{l}\mathrm{Cd}, \mathrm{C} \\
\mathrm{Cu}, \mathrm{Pb} \\
\mathrm{Al}\end{array}$ & $\begin{array}{l}\text { Deuteron Stripping at } 1.69 \\
\text { GeV/c. I. Proton Distri- } \\
\text { butions }\end{array}$ \\
\hline
\end{tabular}

Nucl Phys A145, 337
(1970) J.Gindler
et al

Ukr Fiz Zh 14,1919

(1969)

Yu.A.Berezhnoi,

M.V.Evlanov

Lyon Univ (France)

Thesis, LYCEN-7014

(1970) N.Chevarier

At Energ (USSR)

37,480(1974)

V.S.Barashenkov

et al

Dubna report JINR .

P2-7848(1974)

V.S.Barashenkov

et al

Nucl Instr Meth

106,89(1973)

R.Madey, F.M.Waterman

Nucl Phys A178,

488 (1972)

L.M.C.Dutton

Dubna report JINR-

P2-5261(1970)

K.K.Gudima et al

Nucl Phys A161,105

(1971) R.K.Tandon fission

elastic

scattering,

spallation

excitation

function

excitation

functions, nuclear cascades, multiplicity

cascade showers, inelastic

scattering

neutron spectra

neutron angular distribution

nuclear emulsions

proton angular distributions 
$\begin{array}{ll}27 \mathrm{Al} & \text { Deuteron Spallation in Pulsed } \\ 12 \mathrm{C} & \text { Approximation }\end{array}$

Excitation Functions for Radioactive Nuclides Produced by Deuteron-Induced Reactions in Iron

Mechanism of Interaction of Fast Deuterons with Nuclei

$\mathrm{Be}$

Al,C,Co

$\mathrm{Cu}, \mathrm{Pd}, \mathrm{Ft}$

Ag,Ta

$\mathrm{Al}, \mathrm{Be}, \mathrm{C}$
$\mathrm{Co}, \mathrm{Cu}, \mathrm{Ta}$

Al,Cd.C

$\mathrm{Cu}, \mathrm{Pb}$

B,C

$\mathrm{Cu}, \mathrm{Au}$

C

$\mathrm{Bi}, \mathrm{Pq}$

$\mathrm{Ta}, \mathrm{W}$

Neutrons

Fast Neutron Production with $54 \mathrm{MeV}$ Deuterons

of-Flight Method at $50 \mathrm{MeV}$

Attenuation Cross-Sections

for $650 \mathrm{MeV}$ Deuterons

Absolute Photon Yields of Light Elements by Deuteron Bombardment

of Nuclear Fields

Deuteron-Carbon Elastic

Scattering at $650 \mathrm{MeV}$

Neutrons from the Deuteron
Ukr Fiz Zh $\underline{14,699}$

(1969)

Yu.A.Berezhnoi, L.I.Vinokurov

Phys Rev 179,1104 (1969) J.W.Clark et al

Yadern Fiz $\mathbf{9 . 5 2 8}$ (1969) V.S.Barashenkov et al

Health Phys 13,1237 (1967) J.N.Schultz, W.A.Glass

Nucl Phys A100,537

(1967) G.W.Schweimer

Karlsruhe report

KFK-504(1966)

G.W.Schweimer

Nuovo Cimento (i?)

A48, 165(1967)

K.Ruddick, D.G.Ryan

NASA report NASA-

TN-D-3648(1966)

A.J.Caruso, R.A.Walter

Rev of Mod Phys

37,365(1965)

F.Udo

Bombardment of Thick Targets
Phys Lett 16,331

(1965)

L.M.C.Dutton et al

Nucl Instr Methods

35, 138(1965)

R.R.Borchers,

R.M.Wood impulse

approximation

excitation

function

inelastic

scattering, Monte Carlo method, Coulomb and diffractional disintegration

neutron radiation

angular

distributions

stripping

optical model

$\mathrm{x}$-radiation

neutrons,

spectra

elastic scattering

spectra 


\begin{tabular}{|c|c|c|c|}
\hline $\begin{array}{l}\text { Al,C } \\
\mathrm{O}\end{array}$ & $\begin{array}{l}\text { Proton Groups from the Deu- } \\
\text { teron Bombardment of Aluminum, } \\
\text { Sodium, and Manganese }\end{array}$ & $\begin{array}{l}\text { Phys Rev } 79,99 \\
\text { (1950)W.E.Whitehead, } \\
\text { N.P.Heydenburg }\end{array}$ & \\
\hline $\mathrm{Cl}, \mathrm{Au}$ & $\begin{array}{l}\text { The Deuteron Disintegration } \\
\text { in Flight Occurring During the } \\
\text { Scattering of } 26 \text { ard } 23 \mathrm{MeV} \\
\text { Deuterons on Gold and Cops }\end{array}$ & $\begin{array}{l}\text { Nucl Phys } 70,145 \\
\text { (1965) F.Udo, } \\
\text { L.A.Ch.Koerts }\end{array}$ & $\begin{array}{l}\text { neutrons, } \\
\text { spectra }\end{array}$ \\
\hline $12 \mathrm{C}$ & $\begin{array}{l}\text { Velocity Dependent Potential } \\
\text { and Deuteron Stripping } \\
\text { Reactions }\end{array}$ & $\begin{array}{l}\text { Phys Lett } 14,204 \\
\text { (1965) A.Y.F.bul- } \\
\text { Magd, M.El-Nadi }\end{array}$ & $\begin{array}{l}\text { DWBA approxi } \\
\text { mation, stripping }\end{array}$ \\
\hline $\mathrm{Au}$ & $\begin{array}{l}\text { The Proton Yield Due to the } \\
\text { Disintegration of } 26 \text { and } 23 \\
\text { MeV Deuterons in the Coulomib } \\
\text { Field of Nuclei }\end{array}$ & $\begin{array}{l}\text { Nucl Phys } 63,657 \\
\text { (1965) F.Udo et al }\end{array}$ & \\
\hline Th, U & $\begin{array}{l}\text { Interim Feasibility Report on } \\
\text { MTA Mark III }\end{array}$ & $\begin{array}{l}\text { North American } \\
\text { Aviation report } \\
\text { NAA-SR-130(Del) } \\
\text { (19S1)no author }\end{array}$ & neutrons \\
\hline \multirow[t]{2}{*}{$\mathrm{Cr}$} & $\begin{array}{l}\text { Elastic Scattering of Deu- } \\
\text { terons from the Chromium } \\
\text { Isotopes }\end{array}$ & $\begin{array}{l}\text { Nucl Phys } \$ 6,422 \\
\text { (1964) P.T.Andrews } \\
\text { et } 4\end{array}$ & optical model \\
\hline & $\begin{array}{l}\text { The Energy Correlation in } \\
\text { Proton and Nestron Pairs } \\
\text { Formed by the Disintegration } \\
\text { of } 26-\mathrm{MeV} \text { Deuterons by Gold } \\
\text { Nuclei }\end{array}$ & $\begin{array}{l}\text { Phys Lett } 6,343 \\
\text { (1963) F.Udo, } \\
\text { L.A.Ch.Koerts }\end{array}$ & neutrons \\
\hline $\begin{array}{l}\mathrm{Al}, \mathrm{Bi} \\
12 \mathrm{C}, \mathrm{Au} \\
\mathrm{Fe}, \mathrm{Pb}\end{array}$ & $\begin{array}{l}\text { Total-Reaction Cross-Section } \\
\text { Measurements for Charged } \\
\text { Particles }\end{array}$ & $\begin{array}{l}\text { Lawrence Berkeley } \\
\text { Lab report } \\
\text { UCRL-10783(1963) } \\
\text { Thesis B.D.Wilkins }\end{array}$ & \\
\hline $\begin{array}{l}\mathrm{Co}, \mathrm{Cu} \\
\mathrm{Fe}, \mathrm{Ni}\end{array}$ & $\begin{array}{l}\text { Gross Structure of the Neutron } \\
\text { Energy Spectrum and Polariza- } \\
\text { tion in }(d, n) \text { Reactions un } \\
\text { Intermediate Mass Nuclei }\end{array}$ & $\begin{array}{l}\text { Zh Eksperim I Teor } \\
44,1437(1963) \\
\text { I.I.Levintov et al }\end{array}$ & spectra \\
\hline $28 \mathrm{Si}$ & $\begin{array}{l}\text { The Nuclear Siripping Process } \\
\text { under Conditions of Strong } \\
\text { Absorption }\end{array}$ & $\begin{array}{l}\text { Phys Lett 4,148 } \\
\text { (1963) LJ.B.Gold- } \\
\text { fard, M.B.Hooper }\end{array}$ & $\begin{array}{l}\text { neutrons, } \\
\text { stripping }\end{array}$ \\
\hline $235,238 \mathrm{U}$ & $\begin{array}{l}\text { Yields of Krypton Isotbpes } \\
\text { from the Deuteron and Alpha } \\
\text { Bombardment of Heavy Nuclei }\end{array}$ & $\begin{array}{l}\text { Phys Rev } 124,1949 \\
\text { (1961) M.Kaplan, } \\
\text { C.D.Coryell }\end{array}$ & \\
\hline
\end{tabular}


Table II (Cont.)

\begin{tabular}{|c|c|c|c|}
\hline $\begin{array}{l}\mathrm{Al}, \mathrm{Pb} \\
\mathrm{Zn}\end{array}$ & $\begin{array}{l}\text { Production of Tritium in Lead } \\
\text { and Aluminum by High Energy } \\
\text { Protons, Deuterons and Alpha- } \\
\text { Particles }\end{array}$ & $\begin{array}{l}\text { Zhur Eksptl I } \\
\text { Teoret Fiz 40, } 1263 \\
\text { (1961) V.V.Kuzrietso": }\end{array}$ & \\
\hline$C, 160$ & $\begin{array}{l}\text { Study of the Differential } \\
\text { Cross Sections of Deuteron } \\
\text { Stripping Reactions as a } \\
\text { Function of the Incident } \\
\text { Energy }\end{array}$ & $\begin{array}{l}\text { Phys Rev } 123,619 \\
\text { (1961) E.W.Ham- } \\
\text { burger }\end{array}$ & $\begin{array}{l}\text { Born Approxima- } \\
\text { tion,siripping }\end{array}$ \\
\hline $\begin{array}{l}\mathrm{P}_{\mathrm{t}, \mathrm{Ag}} \\
\mathrm{Ta}, \mathrm{Sn}, \mathrm{Zr}\end{array}$ & $\begin{array}{l}\text { Survey of Inelastic Scattering } \\
\text { of Deuterons by Heavy Elements }\end{array}$ & $\begin{array}{l}\text { Phys Kev } 123,283 \\
\text { (1961) B.L.Cohen, } \\
\text { R.E.Price }\end{array}$ & $\begin{array}{l}\text { inelastic } \\
\text { scattering }\end{array}$ \\
\hline${ }^{9} \mathrm{Be}$ & $\begin{array}{l}\text { Measuremeint of Neutron Spectra } \\
\text { from }(d, n) \text { Reactions on Light } \\
\text { Nuclei }\end{array}$ & $\begin{array}{l}\text { Czechoslov J Phys } \\
\text { B10,715(1960) } \\
\text { J.Juna et al }\end{array}$ & fast neutrons \\
\hline $\begin{array}{l}\text { Al, Co,Cu } \\
\text { Ti,V }\end{array}$ & $\begin{array}{l}\text { Elastic Scattering of Deuter- } \\
\text { ons from } \mathrm{Mg}, \mathrm{Al}, \mathrm{Ti}, \mathrm{V}, \mathrm{Cr} \text {, } \\
\mathrm{Co}, \mathrm{Ni} \text {, and } \mathrm{Cu}\end{array}$ & $\begin{array}{l}\text { Phys Rev } 114,1054 \\
\text { (1959) I.Slaus, } \\
\text { W.P.Alford }\end{array}$ & optical model \\
\hline $235,238 U$ & $\begin{array}{l}\text { Excitation Functions of } 235 \mathrm{U} \\
\text { and } 238 \mathrm{U} \text { Bombarded with Helium } \\
\text { and Deuterium Ions }\end{array}$ & $\begin{array}{l}\text { Phys Rev } 114,163 \\
\text { (1959) J.Wing et al }\end{array}$ & \\
\hline $\begin{array}{l}\mathrm{Al}, \mathrm{Cu} \\
\mathrm{Au}\end{array}$ & $\begin{array}{l}\text { Range-Energy Relation of } 12 \mathrm{C} \text {, } \\
14 \mathrm{~N} \text {, and } 16 \mathrm{O} \text { in Aluminum, } \\
\mathrm{Copper} \text {, and Gold at } 50 \text { to } 110 \\
\mathrm{MeV}\end{array}$ & $\begin{array}{l}\text { Zhur Eksptl I Teoret } \\
\text { Fiz 36.936(1959) } \\
\text { Yu.Ts.Oganesyan }\end{array}$ & \\
\hline $\mathrm{Ge}$ & $\begin{array}{l}\text { Lattice Parameter Changes in } \\
\text { Deuteron Irradiated Germanium }\end{array}$ & $\begin{array}{l}\text { Phys Rev } 113,70 \\
\text { (1959) R.O.Simmons }\end{array}$ & radiation effects \\
\hline \multirow[t]{2}{*}{$\begin{array}{l}9 \mathrm{Be} \\
10,11 \mathrm{~B}\end{array}$} & $\begin{array}{l}\text { Angular Distributions from } \\
\text { Deuteron Bombardment of } \\
\text { Beryllium and Boron }\end{array}$ & $\begin{array}{l}\text { Phys Rev } 112,2020 \\
\text { (1958) B.Zzidman, } \\
\text { JM.Fowler }\end{array}$ & $\begin{array}{l}\text { neutrons, } \\
\text { stripping }\end{array}$ \\
\hline & $\begin{array}{l}\text { Recent Total Neutron Yield } \\
\text { Measurements }\end{array}$ & $\begin{array}{l}\text { Berkeley Radiation } \\
\text { report UCRL-1648, } \\
\text { (1951) W.Crandall, } \\
\text { GMillburn }\end{array}$ & \\
\hline $\begin{array}{l}\text { Be,Th } \\
\text { U }\end{array}$ & $\begin{array}{l}\text { Neutron Production at High } \\
\text { Energies }\end{array}$ & $\begin{array}{l}\text { J Applied Physics } \\
\text { 29,698(1958) } \\
\text { W.E.Crandall, } \\
\text { G.P.Millburn }\end{array}$ & $\begin{array}{l}\text { neutron } \\
\text { detection }\end{array}$ \\
\hline $\mathrm{Ta}, \mathrm{U}$ & $\begin{array}{l}\text { Elastic Scattering of Alpha } \\
\text { Particles and Deuterons from } \\
\text { Heavy Nuclei }\end{array}$ & $\begin{array}{l}\text { Phys Rev } 108,1289 \\
\text { (1957) J.R.Rees, } \\
\text { M.B.Sampson }\end{array}$ & $\begin{array}{l}\text { Ruthertiord } \\
\text { scattering }\end{array}$ \\
\hline
\end{tabular}


Al,U

U,Th

$235_{U}$

$232 \mathrm{Th}$

$238 \mathrm{U}$

$10,11 \mathrm{~B}$

Th,U

$\mathrm{Be}, \mathrm{Th}$

$\mathrm{U}$

$\mathrm{U}$

$\mathrm{Cr} \quad$ Nuclear Excitation Functions and Thick Target Yields: $(\mathrm{Cr}$ + d)

${ }^{9} \mathrm{Be}, 12 \mathrm{C}$

$6,7_{\mathrm{Li}}$ cess Section Quarterly Progress Report for April Through June 1953 Section Quarterly Progress 1952

Fission and Capture Events in 24 Inch $\times 24$ Inch x $101 / 8$ Targets Bombarded with 190MeV Deuterons

Excitation Functions for the to $190 \mathrm{MeV}$ ardment of Boron

Total Neutron Yield from and Protons

Fission and Capture Events in 12 Inch $x 12$ Inch Cross Targets Bombarded with 190 Protons Deuterons

Angular Distribution of Inel-
MTA Project. Target and Pro-

MTA Project Target and Process for September through November Inch Deep Uranium Secondary $(d, 2 n)$ and $(d, 4 n)$ Reactions on $238 \mathrm{U}$ and $232 \mathrm{Th}$ from Threshold

Neutrons from Deuteron BombTargets Bombarded ky Deuterons

Section Uranium and Thorium $\mathrm{MeV}$ Deuterons and $340 \mathrm{MeV}$

Neutron Spectra from a Uranium Target Bombarded with $190 \mathrm{MeV}$

Livermore Research Lab report LWS-24730 (1953) A.V.Shelton

Phys Rev 104,703 (1956) P.Kafalas, J.W.Irvine,Jr. astically Scattered Deuterons

Phys Rev 103,1398

(1956) J.W.Haffner
Livermore Research

neutron beams

Lab report

LRL-82

(1954) no author

Livermore Research

Lab report

MTA-30(1953)

no author

Livermore Research

Lab repott

LRL-106(1954)

H.G.Hicks

Livermore Research Lab report MTA-48

(1953)W.W.T.Crane, G.M.Iddings

Aust J Phys 10,268

(1957) J.R.Bird,

R.H.Spear

Berkeley Radiation

Lab report

UCRL-2063(1957)

W.E.Crandall,

G.P.Milltum

Livermore Research

Lab report MTA-6

(1953) H.G.Hicks

optical model stripping, neutron flux

fission

neutrons

neutrons

optical model 


\section{Table II (Cont.)}

$238 \mathrm{U}$

$\mathrm{U}$

Al

$235 \mathrm{U}$

C

$\mathrm{U}$

Al,Au

$\mathrm{U}$

U

$12 \mathrm{C}$
The Ranges of Fragments from

High Energy Fission of Uranium

${ }^{12} \mathrm{C}(\mathrm{HI}, \mathrm{HI}, \mathrm{n}) 11 \mathrm{C}$ and ${ }^{27} \mathrm{Ai}$ (HI,HI,2p alpha)24/va Cross Sections at High Energies

Fission Product Yields of Uranium Bombarded with Deuterons of Various Energies

(20-190-MeV)

The Fate of Deuterons in Solid Aluminum Targets

High-Energy Induced Fission Theory and Application

Cross Sections for Producing High-Energy Neutrons from Carbon Targets Bombarded by Protons, Deuterons, and ${ }^{3} \mathrm{He}$ Particles

Calorimetric Measurement of the Energy Released in a One Range Uranium Cyclotron Target by $189-\mathrm{MeV}$ Deuterons

Analysis of Secondary Particles Resulting from HighEnergy Nuclear Bombardment

The High-Energy Charged Particles from Targets Bombarded by $190 \mathrm{MeV}$ Deuterons

Stripping and Pick-Up Dif- rential Cross Sections
Berkeley Radiation

Lab report UCRL-1244

(Rev)(1951)

E.M.Doutheth,

D.H.Templeton

Phys Rev 101,329

(1956) W.E.Crandall

et al

excitation

functions

Phys Rev 100,1284

(1955) H.G.Hicks

et al

Livermore Research

Thesis R.R.Hart

Livermore Research

Lab report

R.M.Homing et al

Berkeley Radiation

UCRL-1996(1952)

Thesis, L.Schecter
Lab report LRL-83(1954)

R.A.Heckman, C.J.Egan

Berkeley Radiation

Lab report

UCRL-2996(1955)

Phys Rev 97, 184

(1955) L.Schecter

et al

losses

LRL-70(1953)

Lab report

UCRL-2258(1953)

Thesis,R.W Deutsch

Berkeley Radiation

Lab report

Phys Rev 88, 133

(1952) S.T.Butler,

stripping

irradiations

thermal neutrons

fast neutrons

particle tracks

stripping

E.E.Salpeter 


\section{TRITON- AND ${ }^{3}$ He-INDUCED RFACTIONS}

\section{TARGET}

$6 \mathrm{Li}$

$60 \mathrm{Ni}, 92 \mathrm{Zr}$

${ }^{3} \mathrm{He}, 7 \mathrm{Li}$

$51 \mathrm{~V}$

${ }^{40} \mathrm{Ca}$

$59 \mathrm{Co}$

${ }^{27} \mathrm{Al},{ }^{59} \mathrm{Co}$ $197 \mathrm{Au}, 93 \mathrm{Nb}$

$40 \mathrm{Ca}, 24 \mathrm{Mg}$

$58 \mathrm{Ni}, 32 \mathrm{~S}$

$58 \mathrm{Ni}$
TITLE

Possibility of Using the

Reaction of Disintegration of

Light Ions by Nuclei for

Studying the Nuclear Surface

Properties in the Intermediate

Energy Range

Breakup Phenomena in Nuclear Collision Processes with $\mathrm{He}$

Projectiles

Investigation of Light Nuclei

at High Excitation Energies

with Three-Body Break-Up

Reactions

Theoretical Study of Inclusive

Processes induced by Inter-

mediate Energy Light Ions

Spallation of V Induced by 1

$\mathrm{GeV} 12 \mathrm{C}$-Ions and by 258,350

and $910 \mathrm{MeV} 3 \mathrm{He}$ Ions

Anomalous Behaviour of Tritons

from $156 \mathrm{MeV} 6 \mathrm{Li}$ Break-Up

Reaction

${ }^{3} \mathrm{He}$-Induced Reactions on

Cobalt

Breakup of $3 \mathrm{He}$ Projectiles at an Incident Energy of $\mathbf{4 3 . 3}$

$\mathrm{MeV} /$ Nucleon

Peculiarities of Light

Ion-Nucleus Scattering in

Medium-Energy Region

Scattering of $130 \mathrm{MeV}$ Helionts on $58 \mathrm{Ni}$
REFERENCE

DESCRIPTORS

34th Conf on Nucl.

Spect \& Nucl Struc-

ture, Alma-Ata,USSR

(1984)

M.V.Evlanov et al

Rev Mod Phys 57, 147

(1985) R.J.De

Meijer, R.Kamermans

Julich report

JEL-SPEZ-255,p.22

(1984) R.Franke et al

Theoretical Physics report ITF-83-30-R, p.25(1983) Kiev

Instit.,M.V Evlanov et al

Zeit fur Physik

A315,355(1984)

I.R.Haldorsen et al

Lett Nuovo Cimento

38,237(1983)

R Fonte Catania

Nucl Phys A404,77

(1983) R.Miche!,

M.Galas

Phys Rev C27,2389

(1983) A.Djaloeis

et al

Ukr Phys J 27,177

(1982) Y.A.Berezh-

noj, V.V.Pilipenko

Phys Scripta 26,46

(1982) A.Djaloeis

et al spallation

breakup reactions

quasi-elastic

scattering

breakup reactions

inclusive

interactions

inclusive

interactions

quasi-elastic

scattering

knock-out

reacrions

breakup reactions

stripping

diffraction

models,S-matrix

DWBA 
Table III (Cont.)

\begin{tabular}{|c|c|c|c|}
\hline $197 \mathrm{Au}$ & $\begin{array}{l}\text { Measurement of } 197 \mathrm{Au}(\mathrm{t}, \mathrm{xnyp}) \\
\text { Excitation Functions for } 15 \\
\mathrm{MeV}<\mathrm{E}_{\mathrm{t}}<135 \mathrm{MeV} \text { and Analy- } \\
\text { sis in the Framework of the } \\
\text { Hybrid Model }\end{array}$ & $\begin{array}{l}\text { Julich report } \\
\text { JUEL-SPEZ-98(1981) } \\
\text { O.Bousshid }\end{array}$ & $\begin{array}{l}\text { knock-out } \\
\text { reactions }\end{array}$ \\
\hline $\begin{array}{l}24 \mathrm{Mg}, 58 \mathrm{Ni} \\
120 \mathrm{Sn}, 90 \mathrm{Zr}\end{array}$ & $\begin{array}{l}\text { High Energy Helion Scattering. } \\
\text { A Model-Independent Analysis }\end{array}$ & $\begin{array}{l}\text { Nucl Phys A356,97 } \\
\text { (1981) A.Djaloeis, } \\
\text { S.Gopal }\end{array}$ & optical models \\
\hline $\begin{array}{l}40 \mathrm{Ca}, 119 \mathrm{Sn} \\
58 \mathrm{Ni}\end{array}$ & $\begin{array}{l}\text { Elastic Scattering of } 90-120 \\
\mathrm{MeV} 3 \mathrm{He} \text { Particles and an } \\
\text { Unambiguous Optical Potentia: }\end{array}$ & $\begin{array}{l}\text { Nucl Phys A311,161 } \\
\text { (1978) M.Hyaktak } \\
\text { et al }\end{array}$ & optical models \\
\hline $\begin{array}{l}24 \mathrm{Mg}, 90 \mathrm{Zr} \\
120 \mathrm{Sn}, 208 \mathrm{~Pb}\end{array}$ & $\begin{array}{l}\text { Elastic Scattering of } 130 \mathrm{MeV} \\
{ }^{3} \mathrm{He}\end{array}$ & $\begin{array}{l}\text { Nucl Phys } \mathrm{A} 306,221 \\
\text { (1978) A.Djaloeis } \\
\text { et al }\end{array}$ & optical models \\
\hline \multirow[t]{2}{*}{$\begin{array}{l}27 \mathrm{Al}, 209 \mathrm{Bi} \\
23 \mathrm{Ca}, 197 \mathrm{Au} \\
54 \mathrm{Fe}, 181 \mathrm{Ta}\end{array}$} & $\begin{array}{l}\text { Projectile Break-Up and the } \\
\text { Continuum Spectra of the } \\
\text { (3He,d) Reaction }\end{array}$ & $\begin{array}{l}\text { Nucl Phys A311, } 173 \\
\text { (1978) il.Matsuoka } \\
\text { et al }\end{array}$ & $\begin{array}{l}\text { break-up } \\
\text { reactions }\end{array}$ \\
\hline & $\begin{array}{l}\text { Consistent Microscopic and } \\
\text { Macroscopic Analyses of the } \\
\text { Composite Particle Optical } \\
\text { Potential }\end{array}$ & $\begin{array}{l}\text { Nucl Phys A257,264 } \\
\text { (1976) } \\
\text { S.Mukhopadhyay } \\
\text { et al }\end{array}$ & $\begin{array}{l}\text { elastic } \\
\text { scattering }\end{array}$ \\
\hline${ }^{27} \mathrm{Al}$ & $\begin{array}{l}\text { Particle-Hole State Densities } \\
\text { for Calculation of the Multi- } \\
\text { Step Compound Emission }\end{array}$ & $\begin{array}{l}\text { Nucl Phys A435,67 } \\
\text { (1985) } \\
\text { K.Stankiewicz et al }\end{array}$ & $\begin{array}{l}\text { particla-hole } \\
\text { model }\end{array}$ \\
\hline $197 \mathrm{Au}$ & $\begin{array}{l}\text { Inclusive and Exclusive Cross } \\
\text { Sections for the } 3 \text { He Breakup } \\
\text { on } 197 \text { Au. A Test Case for the } \\
\text { DWBA Approach in the Post- } \\
\text { Formulation }\end{array}$ & $\begin{array}{l}\text { Bonn Univ. Ther:is } \\
\text { INIS-MF-9238 } \\
\text { p.98(1983) } \\
\text { J.Kleinfelier }\end{array}$ & $\begin{array}{l}\text { breakup reactions, } \\
\text { inclusive } \\
\text { interactions }\end{array}$ \\
\hline $\begin{array}{l}197 \mathrm{Au} \\
208 \mathrm{~Pb}\end{array}$ & $\begin{array}{l}33 \text { and } 43 \mathrm{MeV}\left({ }^{3} \mathrm{He}, \mathrm{H} \text { I } \mathrm{n} \text { gamma) }\right. \\
\text { Exclusive Reactions on Targets } \\
\text { from } \mathrm{Zr} \text { to } \mathrm{Pb}\end{array}$ & $\begin{array}{l}\text { Nucl Phys A429,259 } \\
\text { (1984) C.A.Fields } \\
\text { et al }\end{array}$ & $\begin{array}{l}\text { breakup reactions, } \\
\text { neutron spectra, } \\
\text { knock-out } \\
\text { reactions }\end{array}$ \\
\hline $165 \mathrm{Ho}$ & $\begin{array}{l}\text { Breakup-Fusion Calculations } \\
\text { of Continuum Spectra of }(h, p) \\
\text { and }(h, d) \text { Reactions at } E_{h}= \\
100 \mathrm{MeV}\end{array}$ & $\begin{array}{l}\text { Phys Rev C30,1349 } \\
\text { (1984) X.Li et al }\end{array}$ & $\begin{array}{l}\text { stripping, } \\
\text { breakup reactions }\end{array}$ \\
\hline
\end{tabular}


$108 \mathrm{Pd}$

118,124 Sn
Ag Compound-Nucleus Lecay Via the Emission of Heavy Nuclei

${ }^{209} \mathrm{Bi}$

$197 \mathrm{Au}$

$27 \mathrm{Al}$

${ }^{59} \mathrm{Co}$

$150 \mathrm{Nd}$

$124 \mathrm{Sn}$

$62 \mathrm{Ni}$

$150 \mathrm{Nd}$

3 rie-Induced Fission of Nuclei $159<\mathrm{A}<232$

Study of the Reactions on $27 \mathrm{Al},{ }^{59} \mathrm{Co}$ and $112 \mathrm{Sn}$ Nuclei by Action of Accelerated ${ }^{3} \mathrm{He}$ Ions with $34.8 \mathrm{MeV}$ Energy with Proton, Deuteron, Triton, and Alpha Particle Emission

Breakdown of the Thermal Moving-Source Description of of Fast Neutron Production in (alpha, $x n)$ and ( $3 \mathrm{He}, \mathrm{xn})$

Reactions

Study on the Neutron Production from the $3 \mathrm{He}+62 \mathrm{Ni}$ Reaction at an Ion Energy of $59 \mathrm{MeV}$
Inclusive 24 to $43 \mathrm{MeV}$ ( $3 \mathrm{He}$, xn gamma) Reaction (n Pd and Sn Targets

Study on the Energy Dependence of Gamma-Quantum Yield in the Interaction of $3 \mathrm{He}$ and $4 \mathrm{He}$ Ions with $96,98 \mathrm{Ru}$

Breakup Process for $100 \mathrm{MeV}$ $3 \mathrm{He}$ Interacting with $165 \mathrm{Ho}$ and 166,167Er Nuclei

Effect of Nuclear Struchure on Bremsstrahlung Spectra of Multiply Charged Particles

Decay Pathways and Entry-State Population in (alpha,xn gamma) and ( ${ }^{3} \mathrm{He}, \mathrm{xn}$ gamma) Reactions on 150 Nd
Nucl Phys A422,296

(1984) C.A.Fields

et al

23rd Conf on Nucl Spect \& Nucl Struct, Moscow, INIS-SU-198 p.336 (1983)R.A.Zarifov et al

Nucl Phys A413,290 (1984) K.Maeda

et al

23rd Conf on Nucl Spect \& Nucl Struch, Moscow, INIS-SU-198 p.277(983) M.Ya.Amus'ya et al

Phys Rev Lett 51 , 2187(1983)

L.G.Sobotka et al

Phys Rev C28, 1217

(1983)

F.D.Becchetti et al

Sov J Nucl Phys

36.19(1982)

N.T.Burtebcev

et al

Phys Lett B114,8: (1982) C.A.Fields et al

31st Conf on Nucl Spect \& Nucl Struct Samarkland,USSR: INIS-MF-6819 p.391 O.V.Bochkarev et al

Nucl Phys A366,38 (1981) C.A.Fields et al knock-out reactions, inclusive interactions, neutron spectra

gamma radiation

gamma spectra, inclusive interactions

Bremsstrahlung

compound-nucleus reactions

fission

knock-out reactions, breakup reactions, exciton model

inclusive interactions

precompoundnucleus emission, neutron spectra

neutron spectra 
$\begin{array}{ll}\mathrm{Al}, \mathrm{Si} & \text { Reactions Induced by } 3 \mathrm{He} \text { and } \\ & 4 \mathrm{He} \text { Ions on Natural } \mathrm{Mg}, \mathrm{Al}, \\ & \text { and } \mathrm{Si}\end{array}$

$27 \mathrm{Al}$

$208 \mathrm{~Pb}$

$62 \mathrm{Ni}$

$27 \mathrm{Al}$

$40 \mathrm{Ca}$

$12 \mathrm{C}$

164Dy

$174 \mathrm{Yb}$

$27 \mathrm{Al}$

${ }^{27} \mathrm{Al}$

${ }^{59} \mathrm{Co}$

$112 \mathrm{Sn}$
$25 \mathrm{Mg}$ Statistical Multistep Com- pound Emission in the $25 \mathrm{Mg}$ (3 $\mathrm{He}, \mathrm{p})^{27} \mathrm{Al}$ Reaction
107 Ag Analysis of the Excitation Function's for ${ }^{3} \mathrm{He}$ - and Alpha- Induced Reactions on $107 \mathrm{Ag}$ and $109 \mathrm{Ag}$

Measurements and Analysis of Proton, Deuteron, Triton Spectra from Reactions on $27 \mathrm{Al}$, ${ }^{59} \mathrm{Co}, 112 \mathrm{Sn}$ Induced by $3 \mathrm{He}$ Ions at about $35 \mathrm{MeV}$

Preequilibrium Processes of Neutron Production in the Helium and Carbon Ion Reactions

Mass Dependence of $3 \mathrm{He}$ Optical Potentialr and Volume Integrals in this Range from Light to Meditm Weight Nuclei. Selection of a 'Physical' Potential Family

Gamma Deexcitation Mechanism of Rare-Earth Compound Nuclei Produced by $(3 \mathrm{He}, x n)$ and (alpha, xn) Reactions

Statistical Multistep Compound Emission in the ${ }^{27} \mathrm{Al}\left({ }^{3} \mathrm{He}, \mathrm{p}\right){ }^{29} \mathrm{Si}$ Reaction

$26 \mathrm{Mg}$
Phys Rev C25,770 (1982)

DJ.Frantsvog et al

31st Conf on Nucl

Spect \& Nucl Struct

Samarkland,USSR

INIS-MF-6819 p.488

(1981) A.N.Boyarkina et al

Bull Acad Sci USSR

10,2192 (1979)

O.V.Bochkarev

et al

Nucl Phys A337,377

(1980) H.J.Trost

et al

J Phys Soc Japan

46,6(1979)

T.Kishimoto et al

Phys Rev C21,816

(1980) R.Bonetti

et al

28th Conf on Nucl

Spect \& Struct of the Atomic Nucleus Alma-Ata,USSR, 1978 INIS-MF-4388,p.179

N.T.Burtebaev et al

J of Physics G4, L71(1978)

A.De Rosa et al

Kaxlsruhe report

KFK-2311(1976)

P.Misaelides

Phys Rev C14,1655

(1976) P.P.Singh

et al gamma radiation

precompoundnucleus emission

precompoundnucleus emissivil, neutron spectra

elastic scattering, light nuclei

gamma radiation

precompoundnucleus emission

energy spectra

compound-nucleus reactions

excitation functions

precompoundnucleus emission, gamma spectra 


\begin{tabular}{|c|c|c|c|}
\hline $40 \mathrm{Ca}$ & $\begin{array}{l}\text { Monte Carlo Calculations of } \\
\text { Two-Neutron Transfer Cross } \\
\text { Sections }\end{array}$ & $\begin{array}{l}\text { Nucl Phys } \mathrm{A205}, 513 \\
\text { (1973) B.F.Bayman, } \\
\text { D.H.Feng }\end{array}$ & $\begin{array}{l}\text { Monte Carlo } \\
\text { method, pickup } \\
\text { reactions }\end{array}$ \\
\hline $\begin{array}{l}40 \mathrm{Ca} \\
58 \mathrm{Ni}\end{array}$ & $\begin{array}{l}\text { Scattering of } 101-\mathrm{MeV} \text { Helions } \\
\text { from } 12 \mathrm{C}, 40 \mathrm{Ca} \text {, and } 58 \mathrm{Ni}\end{array}$ & $\begin{array}{l}\text { Univ of Maryliand } \\
\text { report ORO-3491-21 } \\
\text { pp.21-3C(1973) } \\
\text { D.A.Goldberg, } \\
\text { S.M.Smith }\end{array}$ & $\begin{array}{l}\text { inelastic and } \\
\text { elastic } \\
\text { scattering }\end{array}$ \\
\hline $\begin{array}{l}40 \mathrm{Ca} \\
12 \mathrm{C}, 208 \mathrm{~Pb} \\
6 \mathrm{Li}, 28 \mathrm{Si}\end{array}$ & $\begin{array}{l}\text { Elastic Scattering of } 217 \mathrm{MeV} \\
\text { 3He Particles }\end{array}$ & $\begin{array}{l}\text { Nucl Phys A204,454 } \\
\text { (1973) N.K ..lis }\end{array}$ & elastic scattering \\
\hline $208 \mathrm{~Pb}$ & $\begin{array}{l}\text { High-Energy Alpha and Helium } \\
\text { Inelastic Scattering }\end{array}$ & $\begin{array}{l}\text { J Phys (Paris) } 5 \\
151(1972) \\
\text { B.Tatischeff et al }\end{array}$ & $\begin{array}{l}\text { inelastic } \\
\text { scattering }\end{array}$ \\
\hline${ }^{208} \mathrm{~Pb}$ & $\begin{array}{l}\text { 3He Elastic Scattering from } \\
208 \mathrm{~Pb} \text { and } 209 \mathrm{Bi} \text { at } 71 \mathrm{MeV}\end{array}$ & $\begin{array}{l}\text { Phys Rev C12,87 } \\
\text { (1975) C.B.Fulmer } \\
\text { et al }\end{array}$ & optical models \\
\hline $\begin{array}{l}150,152 \mathrm{Sm} \\
154 \mathrm{Sm}\end{array}$ & $\begin{array}{l}53 \mathrm{MeV} 3 \mathrm{He} \text { Scattering from } \\
\text { Samarium Isotopes }\end{array}$ & $\begin{array}{l}\text { J Phys G (London) } \\
1,358(1975) \text { R.Eagle } \\
\text { et al }\end{array}$ & optical model \\
\hline $56 \mathrm{Fe}$ & $\begin{array}{l}\text { Interaction of Helium Ions } \\
\text { with } 56 \mathrm{Fe} \text { near } 83 \mathrm{MeV}\end{array}$ & $\begin{array}{l}\text { J Phys G (London) } \\
1,334(1975) \\
\text { S.A.Weisrose et al }\end{array}$ & $\begin{array}{l}\text { elast: } \\
\text { scattering, } \\
\text { one-nucleon } \\
\text { transfer reactions }\end{array}$ \\
\hline $56 \mathrm{Fe}$ & $\begin{array}{l}\text { Unambiguous Helion Optical } \\
\text { Potential at } 83 \mathrm{MeV}\end{array}$ & $\begin{array}{l}\text { J Phys G Nucl Phys } \\
\text { 1,L1(1975) } \\
\text { S.A.Weisrose }\end{array}$ & $\begin{array}{l}\text { elastic } \\
\text { scattering }\end{array}$ \\
\hline $\begin{array}{l}9 \mathrm{Be} \\
27 \mathrm{Al}\end{array}$ & $\begin{array}{l}\text { (3He,2n) Excitation Functions } \\
\text { for Some Light Nuclei }\end{array}$ & $\begin{array}{l}\text { Nucl Phys A235, } 11 \\
\text { (1974) C.E.Moss, } \\
\text { C.S.Zaidins }\end{array}$ & $\begin{array}{l}\text { excitation } \\
\text { functions, } \\
\text { gamma spectra }\end{array}$ \\
\hline $\mathrm{Be}$ & $\begin{array}{l}\text { Fast Neutrons Produced by } \\
\text { Bombarding a Beryllium Target } \\
\text { with } 40 \mathrm{MeV} \text { Helium-3 Ions }\end{array}$ & $\begin{array}{l}\text { Phys Med Biol 20, } \\
125(1975) \\
\text { C.J.Parnell et al }\end{array}$ & neutron sources \\
\hline $\begin{array}{l}63 \mathrm{Cu} \\
61,62 \mathrm{Ni}\end{array}$ & $\begin{array}{l}\text { Neutron, Proton, and Alpha- } \\
\text { Particle Emission from } 3 \mathrm{He} \\
\text { Induced Reactions }\end{array}$ & $\begin{array}{l}\text { Nucl Phys A231,64 } \\
\text { (1974) } \\
\text { A.Chevarier et al }\end{array}$ & $\begin{array}{l}\text { emission spectra, } \\
\text { spallation }\end{array}$ \\
\hline
\end{tabular}




\begin{tabular}{|c|c|c|c|}
\hline $\begin{array}{l}197 \mathrm{Au} \\
181 \mathrm{Ta}\end{array}$ & $\begin{array}{l}\text { Analysis of (particle,xn) } \\
\text { Reactions on Tantalum and Gold } \\
\text { II. } 181 \mathrm{Ta}, 197 \mathrm{Au}\left({ }^{3} \mathrm{He}, \mathrm{xn}\right) \mathrm{Ex}- \\
\text { citation Functions and Equi- } \\
\text { librium Statistical Model } \\
\text { Analysis }\end{array}$ & $\begin{array}{l}\text { Nucl Phys A228,175 } \\
\text { (1974) F.Hermes } \\
\text { et al }\end{array}$ & $\begin{array}{l}\text { excitation } \\
\text { functions }\end{array}$ \\
\hline $58 \mathrm{Ni}$ & $\begin{array}{l}\text { Interference of Coulomb and } \\
\text { Nuclear Excitations in the } \\
\text { Inelastic Scattering of } 3 \mathrm{He} \text {, } \\
6 \mathrm{Li} \text {, and } 12 \mathrm{C} \text { lons on the } 58 \mathrm{Ni} \\
\text { Nucleus }\end{array}$ & $\begin{array}{l}\text { Yadern Fiz } 19,1207 \\
\text { (1974) Yu.A.Gluk- } \\
\text { hov et al }\end{array}$ & $\begin{array}{l}\text { inelastic } \\
\text { scattering }\end{array}$ \\
\hline $\begin{array}{l}209 \mathrm{Bi} \\
197_{\mathrm{Au}}\end{array}$ & $\begin{array}{l}\text { Comparison of }{ }^{3} \mathrm{He}, 4 \mathrm{He} \text {, and } \\
12 \mathrm{C} \text {-Induced Nuclear Reactions } \\
\text { in Heavy-Mass T } \mathrm{T} \text { gete at } \\
\text { Medium Excitation Energies } \\
\text { I. Experimental Cross Sections }\end{array}$ & $\begin{array}{l}\text { Phys Rev C9, } 1064 \\
\text { (1974) } \\
\text { J.D.Stickler, } \\
\text { KJ.Hofstetter }\end{array}$ & $\begin{array}{l}\text { excitation } \\
\text { functions }\end{array}$ \\
\hline $\begin{array}{l}209 \mathrm{Bi} \\
197 \mathrm{Au}\end{array}$ & $\begin{array}{l}\text { Comparison of } 3 \mathrm{He},{ }^{4} \mathrm{He} \text {, and } \\
12 \mathrm{C} \text {-Irduced Nuclear Reactions } \\
\text { in Heavy-Mass Targets at } \\
\text { Medium Excitation Energies } \\
\text { II. Reaction Model Calcula- } \\
\text { tions }\end{array}$ & $\begin{array}{l}\text { Phys Rev C9, } 1072 \\
\text { (1974) } \\
\text { K.J.Hofstetter, } \\
\text { J.D.Stickler }\end{array}$ & $\begin{array}{l}\text { statistical, } \\
\text { exciton, hybrid } \\
\text { and optical models }\end{array}$ \\
\hline${ }^{197} \mathrm{Au}$ & $\begin{array}{l}\text { Peripheral Break-Up of }{ }^{3} \mathrm{He} \text { on } \\
197 \mathrm{Au}\end{array}$ & $\begin{array}{l}\text { Proc Int Conf on } \\
\text { Few Particle Prob- } \\
\text { lems in the } \\
\text { Nuclear Interaction } \\
\text { Aug 28,1972 Los } \\
\text { Angeles, Ca } \\
\text { W.Hermsen et al }\end{array}$ & $\begin{array}{l}\text { peripheral } \\
\text { collisions, } \\
\text { recoils }\end{array}$ \\
\hline $\mathrm{Pb}$ & $\begin{array}{l}\text { Study of }\left({ }^{3} \mathrm{He}, \mathrm{xn}\right),\left({ }^{3} \mathrm{He}, \mathrm{pxn}\right) \text {, } \\
\text { ( } 3 \mathrm{He}, \text { fission) Nuclear Reac- } \\
\text { tions } \mathrm{Oi} \text {.06Pb Eetween } 80 \\
\text { and } 205 \mathrm{MeV}\end{array}$ & $\begin{array}{l}\text { Paris Univ, Orsay, } \\
\text { France, Thesis } \\
\text { FRNC-TH-444(1973) } \\
\text { C.Andre }\end{array}$ & $\begin{array}{l}\text { excitation } \\
\text { functions, } \\
\text { total cross } \\
\text { sections }\end{array}$ \\
\hline $\mathrm{Mg}, \mathrm{Al}, \mathrm{Si}$ & $\begin{array}{l}\text { Total Cross Sections and Exci- } \\
\text { tation Functions for } 1 \mathrm{H}, 2 \mathrm{H} \text {, } \\
3 \mathrm{He} \text {, and }{ }^{4} \mathrm{He} \text { on Targets of } \\
\text { Natural } \mathrm{Mg}, \mathrm{Al} \text {, and } \mathrm{Si}\end{array}$ & $\begin{array}{l}\text { Univ of Colorado } \\
\text { report COO-535-693 } \\
\text { pp.30-32(1973) } \\
\text { C.S.Zaidins et al }\end{array}$ & $\begin{array}{l}\text { one-nucleon } \\
\text { transfer,stripping }\end{array}$ \\
\hline $\begin{array}{l}27 \mathrm{Al} \\
9 \mathrm{Be}\end{array}$ & $\begin{array}{l}(3 \mathrm{He}, 2 \mathrm{n}) \text { Reaction Cross Sec- } \\
\text { tions on Several Light Nuclei }\end{array}$ & $\begin{array}{l}\text { Univ of Colorado } \\
\text { report COO-535-693 } \\
\text { pp.22-23(1973) } \\
\text { C.E.Moss, C.S.Zaidins }\end{array}$ & $\begin{array}{l}\text { excitation } \\
\text { functions }\end{array}$ \\
\hline
\end{tabular}




\begin{tabular}{|c|c|c|c|}
\hline $60 \mathrm{Ni}$ & $\begin{array}{l}\text { Numerical Accuracy of } 3 \mathrm{He} \\
\text { Optical-Model Calculations at } \\
70 \mathrm{MeV}\end{array}$ & $\begin{array}{l}\text { J Cotnput Phy = 12, } \\
498(1973) \\
\text { R.R.Doering et al }\end{array}$ & $\begin{array}{l}\text { differential } \\
\text { cross sections, } \\
\text { optical models }\end{array}$ \\
\hline $27 \mathrm{Al}$ & $\begin{array}{l}\text { Importance of Lare - itgle Data } \\
\text { in Optirel-Mojel Analysis of } \\
\text { Heliug Elastic Scattering }\end{array}$ & $\begin{array}{l}\text { Phys Rev C5, } 1969 \\
\text { (1972) C.B.Fulmer, } \\
\text { J.C.Hafele }\end{array}$ & $\begin{array}{l}\text { elastic } \\
\text { scattering, } \\
\text { optical model }\end{array}$ \\
\hline $239-$ & $\begin{array}{l}\text { Mass Distribution in the } \\
\text { Fission of } 238 \mathrm{U} \text { with } 3 \mathrm{He} \text { Ions }\end{array}$ & $\begin{array}{l}\text { J Inorg Nucl Chem } \\
33,2745(1971) \\
\text { E.F.Meyer et al }\end{array}$ & $\begin{array}{l}\text { fission, fission } \\
\text { yield }\end{array}$ \\
\hline $\begin{array}{l}238 \mathrm{U} \\
209 \mathrm{Bi} \\
232 \mathrm{Th}\end{array}$ & $\begin{array}{l}\text { Delayed Neutrons from the Tau- } \\
\text { and Alpha-Induced Fission of } \\
238 \mathrm{U}, 232 \mathrm{Th} \text {, and } 209 \mathrm{Bi}\end{array}$ & $\begin{array}{l}\text { Nucl Phys A173,497 } \\
\text { (1971) E.T.Chulick, } \\
\text { P.L.Reeder }\end{array}$ & delayed rueutrons \\
\hline $181 \mathrm{Ta}$ & $\begin{array}{l}\text { Highly Inelastic Scattering of } \\
\text { Medium-Energy }{ }^{3} \mathrm{He} \text { and }{ }^{4} \mathrm{He} \\
\text { Particles by Heavy Nuclei }\end{array}$ & $\begin{array}{l}\text { Univ uniknown, } U \text {. } \\
\text { microfilms order } \\
\# 70-14,742 \text { Thesis } \\
\text { (1970) G.M.Chenevert }\end{array}$ & $\begin{array}{l}\text { inelastic } \\
\text { scattering }\end{array}$ \\
\hline $63,65 \mathrm{Cu}$ & $\begin{array}{l}\text { Excitation Functions for }{ }^{3} \mathrm{He} \\
\text { Induced Nuclear Reactions in } \\
\mathrm{Cu}\end{array}$ & $\begin{array}{l}\text { Nucl Phys A152,419 } \\
(1970) \text { N.W.Golchert } \\
\text { et al }\end{array}$ & $\begin{array}{l}\text { excitation } \\
\text { functions }\end{array}$ \\
\hline $56 \mathrm{Fe}$ & $\begin{array}{l}\text { Collective-Model Analysis of } \\
\text { the Inelastic Scattering of } 53 \\
\text { and } 33 \mathrm{MeV} \text { Helions by } 56 \mathrm{Fe}\end{array}$ & $\begin{array}{l}\text { Nucl Phys A202,421 } \\
\text { (19ND) } \\
\text { C.J.Marchese et al }\end{array}$ & $\begin{array}{l}\text { inelastic } \\
\text { scattering }\end{array}$ \\
\hline \multirow[t]{2}{*}{$56 \mathrm{Fe}$} & $\begin{array}{l}\text { Excitation Functions for } \\
\text { Helion-Induced Reactions in } \\
\text { Iron }\end{array}$ & $\begin{array}{l}\text { Phys Rev C6, } 1720 \\
\text { (1972) C.B.Fulmer } \\
\text { et al }\end{array}$ & $\begin{array}{l}\text { nuclear reaction } \\
\text { kinetics }\end{array}$ \\
\hline & $\begin{array}{l}\text { Energy Dependence of the }{ }^{3} \mathrm{He} \\
\text { Optical Potential }\end{array}$ & $\begin{array}{l}\text { Phys Rev Lett 29, } \\
\text { 660(1972) } \\
\text { CJ.Marchese et al }\end{array}$ & $\begin{array}{l}\text { slastic } \\
\text { scattering, } \\
\text { optical model, } \\
\text { intermediate mass } \\
\text { nuclei }\end{array}$ \\
\hline $56 \mathrm{Fe}$ & $\begin{array}{l}\text { Optical-Model Analysis of } \\
\text { Elastic Scattring of } 53.4 \\
\text { MeV Helions from } 56 \mathrm{Fe}\end{array}$ & $\begin{array}{l}\text { Nucl Phys A191,627 } \\
\text { (1972) } \\
\text { C.J.Marchese et al }\end{array}$ & $\begin{array}{l}\text { elastic } \\
\text { scattering, } \\
\text { optical model }\end{array}$ \\
\hline
\end{tabular}


$48 \mathrm{Ca}$

$27 \mathrm{Al}, 28 \mathrm{Si}$

$52 \mathrm{Cr}, 58 \mathrm{Ni}$

$60,62 \mathrm{Ni}$

$181 \mathrm{Ta}$

$186 \mathrm{~W}$

$208 \mathrm{~Pb}$
Recoil Studies of the Interaction of 48 Ca with 18 to 36 $\mathrm{MeV} 4 \mathrm{He}$ lons and 5 to $43-\mathrm{MeV}$ 3 He lons

Dosimetric and Microdosimetric Investications on High L.E.T. Particle Bearns

COMPD: A Computer Program for the Calculation of the Excitation Functions by Means of Monte Carlo Method

Elastic Scattering of $3 \mathrm{He}$ Ions on Light and Intermediate Nuclei

Comparison of Reactions Induced by Medium-Energy $3 \mathrm{He}$ and 4He Particles on Heavy Nuclei
Nucl Phys A153,225

(1970) B.M.Gordon

Centre de Fonteray

Aux-Roses (France) report CEA-R-4655(1975)

A.Bridier, P.Fache

Tokoyo Univ report INS-TCH-10(1974)

K.Komura, S.Tánaka

Yadern Fiz 9,1173

(1969) K.P.Artemov

et al

No university information given Thesis (1968)

N.E.Scott recoils

fast neutrons, microdosimetry

compound-nucleus reactions,

Monte Carlo method

elastic

scattering, optical model

reactions $\left({ }^{3} \mathrm{He}\right.$, $x p),(3 \mathrm{He}, \mathrm{p} 2 \mathrm{n})$, ( $\left.{ }^{3} \mathrm{He}, \mathrm{xr}\right)$, excitation

functions 


\section{ALPHA-PARTICLE INDUCED REACTIONS}

TARGET

\begin{tabular}{|c|c|}
\hline & $\begin{array}{l}\text { Correlated Emission of } \\
\text { Particles in Alpha Induced } \\
\text { Reactions }\end{array}$ \\
\hline $238 \mathrm{U}$ & $\begin{array}{l}\text { Fast Nucleon Emission in High } \\
\text { Energy Alpha Scattering from } \\
\text { Heavy Nuclei }\end{array}$ \\
\hline $\begin{array}{l}27 \mathrm{Al}, 40 \mathrm{Ca} \\
12 \mathrm{C}, 208 \mathrm{~Pb}\end{array}$ & $\begin{array}{l}\text { Mass and Energy Dependence of } \\
\text { the Alpha-Particle Optical } \\
\text { Model Potential in the Energy } \\
\text { Range } 90-172 \mathrm{MeV} \text { and in the } \\
\text { Mass Number Range } 12-208\end{array}$ \\
\hline $\begin{array}{l}209 \mathrm{Bi} \\
165 \mathrm{Ho}_{4} 59 \mathrm{Ni} \\
181 \mathrm{Ta}\end{array}$ & Alpha Induced Reaction: \\
\hline $\begin{array}{l}119 \mathrm{Sn} \\
92 \mathrm{Zr}\end{array}$ & $\begin{array}{l}\text { Neutron Decay of States } \\
\text { Excited in Alpha Inelastic } \\
\text { Scattering }\end{array}$ \\
\hline $238 \mathrm{U}$ & $\begin{array}{l}\text { Importance of Fast Nucleon } \\
\text { Emission in } 172 \mathrm{MeV} \text { Alpha } \\
\text { Scattering from } 238 \mathrm{U}\end{array}$ \\
\hline
\end{tabular}

$55_{\mathrm{M} ., 51 \mathrm{~V}}$

$93 \mathrm{Nb}, 27 \mathrm{Al}$

197 Au

${ }^{59} \mathrm{Co}$

TITLE
REFERENCE

Workshop on Coinci-

dent Particle

Emission from

Continuum States,

Bad Honnef(W.Germany)

INIS-MF-9280(1984)

E.Gadioli et al

INIS-MF-9280(1984)

(Ahsts) P.Decowski

et al

Acta Phys Polonica

B 12,703(1981)

H.Dabrowski,

L.Freindl

Zeit fur Physik

A321, 107(1985)

E.Gadioli et al

Phys Rev C30,1352

(1984) E.Gladioli

et al

Phys Lett B141,49

(1984) P.Decowski

et al

Int Corar on Nucl

D:ia for Sci \& Tech

Antwerp,Belgium

(1982) p. 599

R.Michel et al

Zeit fur Physik

A316,309(1984)

A.Niita

Zeit fur Physik

A317, 155(1984)

E.Gadioli et a!
DESCRIPTORS

precompoundnucleus emission, inclusive interactions

precompoundnucleus emission, quasi-elastic scattering

optical models, nuclear potential

neutron spectra

neutron emission

quasi-elastic scattering, pre-compoundnucleus emission

excitation

function

precompoundnucleus, inclusive interactions

precompoundnucleus emission, quasi-elastic scattering 


\begin{tabular}{|c|c|c|c|}
\hline${ }^{197} \mathrm{Au}$ & $\begin{array}{l}\text { Excitation Functions of the } \\
197 \mathrm{Au}(\text { alpha,2p) } \\
199 \mathrm{Au} \text { and } \\
197 \mathrm{Au}(\text { alpha,2n) } \\
199 \mathrm{~T} \text { Reac- } \\
\text { tions }\end{array}$ & $\begin{array}{l}\text { Nucl Phys } \underline{A 413}, 432 \\
\text { (1984) O.Hashimoto } \\
\text { et al }\end{array}$ & $\begin{array}{l}\text { breakup reactions } \\
\text { gamma spectra }\end{array}$ \\
\hline $16.5 \mathrm{Ho}, 58 \mathrm{Ni}$ & $\begin{array}{l}\text { Breakup-Fusion Descritrion } \\
\text { of Nonequilibrium Protons } \\
\text { From (alpha,p) Reactions }\end{array}$ & $\begin{array}{l}\text { Phys Lett B135,333 } \\
\text { (1984) T.Udagawa } \\
\text { et al }\end{array}$ & $\begin{array}{l}\text { inclusive } \\
\text { interactions, } \\
\text { breakup reactions }\end{array}$ \\
\hline $197 \mathrm{Au}$ & $\begin{array}{l}\text { Proton Emission in Alpha- } \\
\text { Induced Reactions at } 43 \mathrm{MeV} / \\
\text { Nucleon }\end{array}$ & $\begin{array}{l}\text { Nucl Phys A408, } 359 \\
\text { (1983) B.Ludewigt } \\
\text { et al }\end{array}$ & $\begin{array}{l}\text { knock-out } \\
\text { reactions, } \\
\text { inclusive } \\
\text { interactions }\end{array}$ \\
\hline $93 \mathrm{Nb}$ & $\begin{array}{l}\text { Model for Alpha Particle } \\
\text { Induced Nuclear Reactions: } \\
93 \mathrm{Nb} \text { (alpha,x alpha ypzn) from } \\
40-140 \mathrm{MeV}\end{array}$ & $\begin{array}{l}\text { Phys Rev C29,76 } \\
\text { (1984) E.Gadioli } \\
\text { et al }\end{array}$ & $\begin{array}{l}\text { compound- } \\
\text { nucleus } \\
\text { reactions }\end{array}$ \\
\hline $\begin{array}{l}27 \mathrm{Al}, 58 \mathrm{Ni} \\
62 \mathrm{Ni}, 28 \mathrm{Si}\end{array}$ & $\begin{array}{l}\text { Study of Continuous Spectra } \\
\text { of Scattered Alpha and } 6 \mathrm{Li} \\
\text { Particles at } 26 \mathrm{MaV} / \mathrm{A} \text { by } \\
\text { Detection of Coincident Gamma } \\
\text { Radiation }\end{array}$ & $\begin{array}{l}\text { INIS-MF-8251(1981) } \\
\text { Heidelberg Univ. } \\
\text { Thesis, W.Nitsche }\end{array}$ & gamma spectra \\
\hline $\begin{array}{l}162,104 \mathrm{Dy} \\
174 \mathrm{Yb}\end{array}$ & $\begin{array}{l}\text { Neutron-M } M_{4} \text { :tiplicity Distri- } \\
\text { butions for (apha,xn gamma) } \\
\text { Reactions with } E=50-120 \\
\text { MeV and the Pre-Equilibrium } \\
\text { Processes }\end{array}$ & $\begin{array}{l}\text { Phys Rev C28, } 635 \\
\text { (1983) KMaeda } \\
\text { et al }\end{array}$ & $\begin{array}{l}\text { multiplicity, } \\
\text { neutrons, } \\
\text { exciton model }\end{array}$ \\
\hline $208 \mathrm{~Pb}$ & $\begin{array}{l}\text { One- and Non-Coplanar Two- } \\
\text { Particle Inclusive Cross- } \\
\text { Sections from the } 4 \mathrm{He}+\mathrm{Pb} \\
\text { Reaction at } 150 / \mathrm{MeV} \text { Nucleon }\end{array}$ & $\begin{array}{l}\text { Saclay report } \\
\text { CEA-N-2296 p.149 } \\
(1982) K . C h a m c h a m \\
\text { et al }\end{array}$ & $\begin{array}{l}\text { deuterons, } \\
\text { energy spectra }\end{array}$ \\
\hline $93 \mathrm{Nb}$ & $\begin{array}{l}\text { Reactions Induced on } 93 \mathrm{Nb} \text { by } \\
\text { Alpha Particles up to } 140 \mathrm{MeV}\end{array}$ & $\begin{array}{l}\text { Saclay report CEA- } \\
\text { N-2296 p.146(1982) } \\
\text { E.Gadioli et al }\end{array}$ & neutrons \\
\hline $\begin{array}{l}197 \mathrm{Au} \\
164 \mathrm{Dy}\end{array}$ & $\begin{array}{l}\text { Is Neutron Evaporation From } \\
\text { Highly Excited Nuclei a } \\
\text { Poisson Random Process }\end{array}$ & $\begin{array}{l}\text { Zeit fur Physik } \\
\text { A307,141(1982) } \\
\text { M.H.Simbel }\end{array}$ & $\begin{array}{l}\text { neutron emission, } \\
\text { distribution } \\
\text { functions }\end{array}$ \\
\hline $\begin{array}{l}93 \mathrm{Nb} \\
181 \mathrm{Ta}\end{array}$ & $\begin{array}{l}\text { Investigation of Alpha-Induced } \\
\text { Reactions on Niobium and } \\
\text { Tantallum. Pt. } 1\end{array}$ & $\begin{array}{l}\text { Zeit fur Physik } \\
\text { A308,301(1982) } \\
\text { J.Ernst et al }\end{array}$ & breakup reaction \\
\hline
\end{tabular}




\begin{tabular}{|c|c|c|c|}
\hline${ }^{54} \mathrm{Fe}$ & $\begin{array}{l}\text { Mechanisms for Composite } \\
\text { Particle Production in Nuclear } \\
\text { Reactions Using (alpha, alpha' } \\
\text { x) Reactions Up To } 40 \mathrm{MeV} \text { ' } \\
\text { Nucleon }\end{array}$ & $\begin{array}{l}\text { Phys Rev C26,411 } \\
\text { (1982) H.Machner } \\
\text { et al }\end{array}$ & composite models \\
\hline $\mathrm{Br}$ & $\begin{array}{l}\text { Total Reaction Cross-Sections } \\
\text { Determined from Elastic Scat- } \\
\text { tering of Alpha-Particles on } \\
\mathrm{Br}\end{array}$ & $\begin{array}{l}\text { Lett Nuuvo Cim Soc } \\
\text { Ital } 25,481(1979) \\
\text { G.Lanzano et al }\end{array}$ & $\begin{array}{l}\text { total cross } \\
\text { sections }\end{array}$ \\
\hline $12 \mathrm{C}$ & $\begin{array}{l}\text { Dominance of Nucleon-Nucleon } \\
\text { Interactions in Alpha }+12 \mathrm{C} \\
\text { Total Reaction Cross Sections }\end{array}$ & $\begin{array}{l}\text { Phys Rev C26,301 } \\
\text { (1982) R.M.Devries } \\
\text { et al }\end{array}$ & $\begin{array}{l}\text { nucleon-nucleon } \\
\text { interactions }\end{array}$ \\
\hline $\begin{array}{l}166 \mathrm{Er} \\
192 \mathrm{Os} \\
122 \mathrm{Te}\end{array}$ & $\begin{array}{l}\text { Decay of Compound Nuclei } \\
\text { Following Alpha-Particle and } \\
12 \mathrm{C} \text { induced Reactions }\end{array}$ & $\begin{array}{l}\text { Zeit fur Physik } \\
\text { 301,35(1981) } \\
\text { S.A.Hjorth }\end{array}$ & $\begin{array}{l}\text { gamma spectra, } \\
\text { multiplicity }\end{array}$ \\
\hline $\begin{array}{l}197 \mathrm{Au} \\
93 \mathrm{Nb} \\
232 \mathrm{Th}\end{array}$ & $\begin{array}{l}\text { Interpretation of the Contin- } \\
\text { uous Spectrum of Secondary } \\
\text { Alpha Farticles in Alpha } \\
\text { Induced Reactions }\end{array}$ & $\begin{array}{l}\text { Zeit fur Physik } \\
\text { A299,1(1981) } \\
\text { E.Gadioli, E.G.Erba }\end{array}$ & exciton model \\
\hline $\begin{array}{l}197 \mathrm{Au} \\
58 \mathrm{Ni}\end{array}$ & $\begin{array}{l}\text { Target Dependence of Isotope } \\
\text { Y'seld in Alpha-Induced } \\
\text { Reactions at } E=43 \mathrm{MeV} / \mathrm{A}\end{array}$ & $\begin{array}{l}\text { Phys Lett B108,15 } \\
\text { (1982) B Ludewigt } \\
\text { et al }\end{array}$ & $\begin{array}{l}\text { inclusive } \\
\text { interactions }\end{array}$ \\
\hline $\begin{array}{l}27 \mathrm{Al} \\
181 \mathrm{Ta}\end{array}$ & $\begin{array}{l}\text { Proton Inclusive Cross } \\
\text { Sections from } 720 \mathrm{MeV} \text { Alpha- } \\
\text { Nucleus Reactions }\end{array}$ & $\begin{array}{l}\text { Nucl Phys A362,431 } \\
\text { (1981) KR.Cordell } \\
\text { et al }\end{array}$ & $\begin{array}{l}\text { inclusive } \\
\text { interactions, } \\
\text { proton spectra }\end{array}$ \\
\hline \multirow[t]{2}{*}{${ }^{28} \mathrm{Si}$} & $\begin{array}{l}\text { Importance of Nucleon Knock- } \\
\text { Out in Inelastic Alpha and } \\
\text { 6Li Scattering }\end{array}$ & $\begin{array}{l}\text { Zeit fur Physik } \\
\text { A300, } 109(1981) \\
\text { W.Nitsche et al }\end{array}$ & $\begin{array}{l}\text { inelastic } \\
\text { scattering, } \\
\text { knock-nut } \\
\text { reactions }\end{array}$ \\
\hline & $\begin{array}{l}\text { In Beam Gamma Ray Spectros- } \\
\text { copy with } 100-160 \text { MeV Alpha's } \\
\text { In Medium Light Nuclei }\end{array}$ & $\begin{array}{l}\text { Selected Topics in } \\
\text { Nucl. Structure, } \\
\text { Winter School } \\
\text { Bielsko-Biala, } \\
\text { Poland, INP-1014/PL, } \\
\text { p.381(1978) } \\
\text { C.Signorini }\end{array}$ & $\begin{array}{l}\text { precompound- } \\
\text { nucleus, } \\
\text { intermediate } \\
\text { mass nuclei }\end{array}$ \\
\hline $40 \mathrm{Ca}, 12 \mathrm{C}$ & $\begin{array}{l}\text { Role of the Atractive Nuclear } \\
\text { Potential in Determining } \\
\text { Reaction Cross Sections }\end{array}$ & $\begin{array}{l}\text { J of Physics G7,43 } \\
\text { (1981) D.M.Erink, } \\
\text { G.R.Satchler }\end{array}$ & optical models \\
\hline
\end{tabular}




$\begin{array}{ll}{ }^{162} \mathrm{Dy} & \text { K-Shell Ionization of Dy } \\ { }^{158} \mathrm{Gd} & \begin{array}{l}\text { Atoms Produced in } 158 \mathrm{Gd} \\ \text { (alpha xn) Reactions }\end{array}\end{array}$

160Gd Gamma-Ray Multiplicities, $150 \mathrm{Nd} \quad$ Energies and Entry Lines Observed in Equilibrium and PreEquilibrium Decay Following Alpha.Induced Reactions

$\begin{array}{ll}197 \mathrm{Au}, 93 \mathrm{Nb} & \begin{array}{l}\text { Interpretation of the Contin- } \\ \text { uous Sfectrum of Secondary } \\ \text { Alpha Particles in Alpha } \\ \text { Induced Reactions }\end{array} \\ & \\ 54 \mathrm{Fe} & \begin{array}{l}\text { Investigation of (alpha, } \\ \text { alpha' } \mathrm{x}) \text { Reactions on } 54 \mathrm{Fe} \\ \text { from } 60 \text { to } 160 \mathrm{MeV} \text { Bombarding } \\ \text { Energy }\end{array}\end{array}$

$\begin{array}{ll}158,160 \mathrm{Gd} & \begin{array}{l}\text { K-Shell Internal Conversion } \\ \text { Yields in Light Dy Nuclei } \\ \text { Populated in Alpha and } \\ 12 \text { C-Induced Reactions }\end{array} \\ \mathrm{Zr} & \begin{array}{l}\text { Effect of the Phase Space } \\ \text { Factor in the Breakup of } \\ \text { Composite Particles }\end{array} \\ \text { Dy,Gd } & \begin{array}{l}\text { Multipolarities of Quasi- } \\ \text { continuum Gamma-Ray Cas- } \\ \text { cades Following (alpha, xn) } \\ \text { and (H.I.,xn) Reactions }\end{array}\end{array}$
${ }^{209} \mathrm{Bi} \quad$ Study of Fission from $\mathrm{Ag}, \mathrm{Au}$, ${ }_{197} \mathrm{Au} \quad \mathrm{Pb}, \mathrm{Bi}$, Th and U Targets 232Th,238U Induced by $640 \mathrm{MeV}$ Alpha- Particles

${ }^{59} \mathrm{Co}$
Nukleonika 24,395 (1979) D.Chmielewska et al

Nukleonika 24,425 (1979)

MJ.A.De Voigt et al

Zeit fur Physik

A299, 1(1981)

E.Gadioli, E.E.Gadioli

JUEL-SPEZ-72 p.29

(1979) Muenster Univ.(W.Germany) (Annual Report)

Int Conf on Struct

of Medium-Heavy

Nuclei,Rhodos,Greece

(1980) p.207

D.Chmielewska et al

Phys Rev C23, 1839

(1981)G.Paic,

B.Antolokovic

Int. Workshop on

Gross Properties of

Nuclei \& Nuclear

Excitations, Hirschegg,

Austria, INKA-CONF-79-

001-000, p.75(1979)

Z.Sujkowski et al

Nucl Instr \& Meth

173, 157(1980)

M.Debeauvais,

J.Tripier

Nucl Phys A338, 167

(1980) R.Michel,

G.Brinkmann

Nucl Phys A333,285

(1980) J.P.Bondorf

et al x-ray spectra, ionization

gamma cascades

exciton model

precompound-

nucleus emission

K-conversion, cross sections

phase space

gamma cascades

fission fragments

precompoundnucleus,knockout reactions

prompt neutrons 


\begin{tabular}{|c|c|c|c|}
\hline $27 \mathrm{Al}, 28 \mathrm{Si}$ & $\begin{array}{l}\text { Nuclear Gamma Rays From } 720 \text { - } \\
\text { MeV Alpha-Induced Reactions } \\
\text { on } 27 \mathrm{Al} \text { and } 28 \mathrm{Si}\end{array}$ & $\begin{array}{l}\text { Phys Rev C22,1612 } \\
\text { (1980) B.J Lieb } \\
\text { et al }\end{array}$ & $\begin{array}{l}\text { prompt gamma } \\
\text { radiation, total } \\
\text { cross sections }\end{array}$ \\
\hline Dy,Gd & $\begin{array}{l}\text { K-Shell Ionization of Heavy } \\
\text { Atoms Produced in (alpha,xn) } \\
\text { Nuclear Reactions }\end{array}$ & $\begin{array}{l}\text { Phys Rev A21, } 1764 \\
\text { (1980)Z.Sujkowski, } \\
\text { D.Chmielewska }\end{array}$ & $\begin{array}{l}\text { ionization, } \\
\text { K-shell }\end{array}$ \\
\hline $154,160 \mathrm{Gd}$ & $\begin{array}{l}\text { Cross Sections of the (alpha, } \\
x n) \text { Reaction on } 154 \mathrm{Gd} \text { and } \\
160 \mathrm{Gd} \text { Nuclei in the } E=47 \text {. } \\
130 \mathrm{MeV} \text { Energy Range }\end{array}$ & $\begin{array}{l}\text { Bull Acad Sci USSR } \\
\text { 42,2369(1978) } \\
\text { D.Chmielewska et al }\end{array}$ & gamma radiation \\
\hline $160 \mathrm{Gd}$ & $\begin{array}{l}\text { Experimental Investigation of } \\
\text { the Quasi-Continuum Gamma-Ray } \\
\text { Cascades Following } 160 \mathrm{Gd} \\
\text { (alpha,xn) } 164-\mathrm{X}_{\text {Dy Reactions }}\end{array}$ & $\begin{array}{l}\text { Nucl Phys A323,317 } \\
\text { (1979)M.J.A.DeVoigt } \\
\text { et al }\end{array}$ & $\begin{array}{l}\text { precnmpound- } \\
\text { nL: } \quad \text { in ana } \\
\text { cascades }\end{array}$ \\
\hline $90 \mathrm{Zr}$ & $\begin{array}{l}\text { Projectile Fragmentation Pro- } \\
\text { cesses in 35-MeV/AMU (alpha, } \\
\text { xy) Reactions }\end{array}$ & $\begin{array}{l}\text { Phys Rev Lett } 43 \text {. } \\
\text { 1862(1979) } \\
\text { R.W.Koontz et al }\end{array}$ & $\begin{array}{l}\text { evaporation } \\
\text { model }\end{array}$ \\
\hline $\begin{array}{l}209 \mathrm{Bi} \\
233 \mathrm{U}\end{array}$ & $\begin{array}{l}\text { Reaction Mechanism Studies } \\
\text { with } 7-35 \mathrm{MeV} / \mathrm{Nucleon} 4 \mathrm{He} \\
\text { Ions Incident on Heavy Target } \\
\text { Nuclei }\end{array}$ & $\begin{array}{l}\text { Phys Rev C20,1716 } \\
\text { (1979) W.G.Meyer } \\
\text { et al }\end{array}$ & $\begin{array}{l}\text { precompound- } \\
\text { nucleus emission }\end{array}$ \\
\hline $\begin{array}{l}27 \mathrm{Al} \\
209 \mathrm{Bi} \\
90 \mathrm{Zr}\end{array}$ & $\begin{array}{l}\text { Alpha-Particle Breakup at } \\
\text { Incident Energies of } 20 \text { and } \\
40 \mathrm{MeV} / \mathrm{Nucleon}\end{array}$ & $\begin{array}{l}\text { Phys Rev C20, } 1284 \\
\text { (1979) J.R.Wu et al }\end{array}$ & $\begin{array}{l}\text { deuteron spectra, } \\
\text { proton spectra }\end{array}$ \\
\hline $164 \mathrm{Dy}$ & $\begin{array}{l}\text { Pre-Equilibrium Neutron } \\
\text { Emission for the } 164 \mathrm{Dy} \text { (alpha, } \\
\text { xn gamma) } 168 \times \mathrm{Er} \text { Reaction } \\
\text { Studied by n-Gamma Coincidence } \\
\text { Measurements }\end{array}$ & $\begin{array}{l}\text { Phys Rev C20,464 } \\
\text { (1979) H.Sakai } \\
\text { et al }\end{array}$ & $\begin{array}{l}\text { precompound- } \\
\text { nucleus emission }\end{array}$ \\
\hline $160 \mathrm{gd}$ & $\begin{array}{l}\text { Compound and Precompound } \\
\text { Gamma-Ray Entry Lines From } \\
\text { Measured Multiplicities and } \\
\text { Energies in Alpha-Induced } \\
\text { Reactions }\end{array}$ & $\begin{array}{l}\text { Phys Lett B78,401 } \\
\text { (1978) W.J.Ockels } \\
\text { et al }\end{array}$ & $\begin{array}{l}\text { gamma spectra, } \\
\text { precompound- } \\
\text { nucleus emission }\end{array}$ \\
\hline $40,48 \mathrm{Ca}$ & $\begin{array}{l}104 \mathrm{MeV} \text { Alpha Particle and } \\
156 \mathrm{MeV} 6 \mathrm{Li} \text { Scattering and } \\
\text { the Validity of Refined } \\
\text { Folding Model Approaches for } \\
\text { Light Complex Projectile } \\
\text { Scattering }\end{array}$ & $\begin{array}{l}\text { Zeit fur Physik } \\
288,139(1978) \\
\text { Z.Maika et al }\end{array}$ & $\begin{array}{l}\text { elastic } \\
\text { scattering, } \\
\text { optical models }\end{array}$ \\
\hline
\end{tabular}




\begin{tabular}{|c|c|}
\hline $\begin{array}{l}27 \mathrm{Al}, \mathrm{Ag} \\
181 \mathrm{Ta}\end{array}$ & $\begin{array}{l}\text { Back-Angle Proauction of } \\
\text { Light Ions From } 720-\mathrm{MeV} \\
\text { Alpha-Nucleus Col'isions }\end{array}$ \\
\hline \multirow[t]{2}{*}{$\begin{array}{l}27 \mathrm{Al}, 209 \mathrm{Bi} \\
232 \mathrm{Th}\end{array}$} & $\begin{array}{l}\text { Charged Particle Spectra: } 140 \\
\text { MeV Alpha Particle Bombard- } \\
\text { ment of } 27 \mathrm{Al}, 58 \mathrm{Ni}, 90 \mathrm{Zr} \text {, } \\
209 \mathrm{Bi} \text {, and } 232 \mathrm{Th}\end{array}$ \\
\hline & $\begin{array}{l}\text { Multi-Detector Arrangements } \\
\text { for On-Line Measurements of } \\
\text { Gamma-Ray and Neutron } \\
\text { Multiplicities }\end{array}$ \\
\hline $52 \mathrm{Cr}$ & $\begin{array}{l}\text { Cross Sections and Excitation } \\
\text { Functions of } 40 \text { to } 110 \mathrm{MeV} \\
\text { Alpha-Particle Induced Reac- } \\
\text { tions in Natural Chromium }\end{array}$ \\
\hline
\end{tabular}

$150 \mathrm{Sm} \quad$ Gamma-Ray Multiplicities in Alpha-Induced Compound and Precomipound Reactions
$40 \mathrm{Ca}, 58 \mathrm{Ni} \quad$ Modified Optical Potential for Elastic Alpha Scattering: Folding Potentials and Energy Dependence

$\begin{array}{ll}27 \mathrm{Al}, \mathrm{Ag} & \begin{array}{l}\text { Correlated Energy Spectra of } \\ \text { Light Fragments from } 720-\mathrm{MeV}\end{array} \\ 181 \mathrm{Ta} & \text { Alpha-Induced Reactions } \\ 40 \mathrm{Ca}, 59 \mathrm{Co} & \begin{array}{l}\text { Gamma Spectroscopy with High } \\ \text { Energy Alpha Particles }\end{array}\end{array}$

$\mathrm{C}, \mathrm{Ce}, \mathrm{Hg} \quad$ In Search of Heavy, Fast F̄ragments or Nuclear Recoils in Reactions of $21 \mathrm{GeV}$ Neutrons and $700 \mathrm{MeV}$ Alphas with $\mathrm{Hg}$, $\mathrm{Ta}, \mathrm{Ce}, \mathrm{Y}, \mathrm{Cu}, \mathrm{Mn}$ and $\mathrm{Ca}$

\begin{tabular}{|c|c|}
\hline $\begin{array}{l}{ }^{59} \mathrm{Co} \\
{ }^{63} \mathrm{Cu}\end{array}$ & 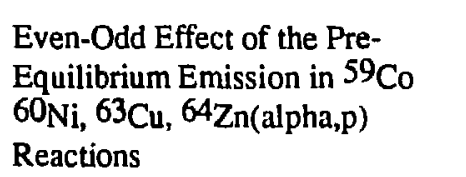 \\
\hline $\begin{array}{l}237 \mathrm{~Np} \\
233,235 \mathrm{U}\end{array}$ & $\begin{array}{l}\text { Pre impound Evaporation Analy- } \\
\text { ses of Excitation Functions } \\
\text { for (alpha,xn) Reactions }\end{array}$ \\
\hline
\end{tabular}

Phys Rev C19,913

(19?9) S.T.Thomton

et al
Phys Rev C19,659
(1979) J.R.Wu
et al

Nucl Instr \& Meth $150,483(1978)$

A.Kerek et al

Radiochimica Acta

24,11(1977)

A.Cavallero et al

Zeit fur Physik

A284,271(1978)

M.Ogawa et al

Phys Rev C17,1322

(1978) A.A.Cowley

Phys Rev Lett 40 ,

1433(1978)

R.Doering et al

Saclay report

CEA-N-1861 p.51-53

(nd) O.Artun et al

Helv Phys Acta 48, 343(1975)

H.W.Von Reist et al

Chinese Physics

4,113(1984)

X.Lu et al

Phys Rev C29,1678

(1984) M.Blann,

T.T.Komoto multiple

scattering

breakup reactions charged particles, energy spectra

gamma cascades multiplicity, neutron detection

excitation functions

multiplicity, neutrons

Woods-Saxon potential

deuteron spectra

gamma radiation

energy transfer

precompoundnucleus emission, exciton model

precompoundnucleus emission, neutron emission 
$1817 \mathrm{a}$

Gd

$28 \mathrm{Si}$

$40 \mathrm{Ca}$

160

$150 \mathrm{Nd}$

124 Sn

$28 \mathrm{Si}$

$12 \mathrm{C}$

$181 \mathrm{Ta}$

${ }^{159} \mathrm{~Tb}$

121Sb

197 Au
Gamma Spectra in 181 Ta Bombardment oy Alpha Particles

Determination of the AlphaInduced K-Shell Ionization Cross Sections from the Measured Intensity Ratios of $\mathrm{K}$ $X$-Rays to $\left(2+\rightarrow 0^{+}\right)$GammaRays in Even Deformed Nuclei

Analysis of Elastic and Inelastic Scattering of $\mathbf{5 0 . 5}$ MeV Alpha-Particles on $28 \mathrm{Si}$ Nucleus

Microscopic Alpha-Nucleus

Potentials

Exclusive (alpha,2n gamma) Neutron Spectra and the PreEquilibrium Exciton Model

Mechanisms for Reactions of Alpha-Particles on 28Si at $\mathrm{E}_{\text {alpha }}=65 \mathrm{MeV}$ Leading to Three-Body Final States

Energy Dependence of Proton Emission in $4 \mathrm{He}$ and ${ }^{12} \mathrm{C}$ Induced Reactions Between 25 and $84 \mathrm{MeV} / \mathrm{AMU}$

Gamma Spectra in Alpha Bombardment of $181 \mathrm{Ta}$
Bull Acad Sciences

Phys Series 47 ,

849(1983)

V.D.Avchukhov et al

Nucl Instr Meth

219,111(1984)

Z.Sujkowski et al

23rd Conf on Nucl

Spect \& Nucl Struct

Moscow, INIS-SU-

198 p.333(1983)

N.T.Burtebaev et al

Nucl Phys A408,239

(1983) D.Wintgen

et al

Phys Lett B130,350

(1983) E.Betak,

J.Dobes

Nucl Phys A402,15

(1983)

R.J.De Meijer

et al

Phys Lett B120,71

(1983) R.Glasow

et al

32nd Conf on Nucl

Spect \& Nucl Struct

Kiev, INIS-SU-125

p.125(1982)

V.D.Avchukhov

Julich report

JUEL-SPEZ-146

(1981) J.P.Didelez

et al

Nucl Phys A383,25!

(1982)

A.Calboreanu et al gamma spectra

ion-atom

collisions, x-ray spectra

elastic and inelastic

scatrering

elastic scattering

knock-out reactions, neutron spectra

knock-out reactions, inclusive interactions

knock-out reactions

prompt gamma radiation

breakup reactions, charge-exchange reactions

stripping Competition on the (alpha, $n$ ) and (alpha, $2 n$ ) Reactions on Gold and Antimony 
Energy and Angular Momentum Transfers in Equilibrium and Pre-Equilibrium 158Gd(alpha,xn) Reactions

${ }^{59} \mathrm{Co}$

$63 \mathrm{Cu}$

$204,206 \mathrm{~Pb}$

$207,208 \mathrm{~Pb}$

Al

$165 \mathrm{Ho}$

$150 \mathrm{Nd}$

$154,155 \mathrm{Gd}$

$28 \mathrm{Si}$
Entrance Channel Effects in

Nucleon and Alpha Particle

Preequilibrium Decay of $63 \mathrm{Cu}$ and $64 \mathrm{Zn}$

Cross Sections and Thick Target Yields of (alpha, $x n$ )

Reactions on Natural Lead

Average Number of Protons

Knocked-Out from the $\mathrm{C}, \mathrm{Ne}$, $\mathrm{Al}, \mathrm{Cu}, \mathrm{Pb}$ Nuclei by ${ }^{4} \mathrm{He}$

Nuclei with the Momentum of 4.5 GeV/C Nucleon

Improved Anilysis of GammaRay Angular Distributions in (particle,xn) Experiments

Fast Neutron Emission in (alpha,2n) Reactions: A Neutron Skin Effect

K-Shell Ionization of Dy Atoms Produced in (alpha,xn) Nuclear Reactions

Importance of Nucleon KnockOut In Inelastic Alpha and $6 \mathrm{Li}$ Scattering
Nucl Phys A379,160 (1982)

MJ.A.De Voigt et al

Phys Rev C26,113

(1982)

R.Scherwinski et al

Radiochem \& Radioanalytical Lett 50 .

211(1982)

C.Wasilevsky et al

Yad Fiz 6,33,1568

(1981)

M.K.H.Anikina

et al

Nucl Instr Meth 190, 19(1981)

V.A.Ionescu et al

Phys Lett B106,453 (1981) C.A.Fields et al

Nucl Phys A366, 142(1981)

1 Jhmielewska

et al

Lawrence Berkeley

Lab report LBL12652 p687(1981)

Sth High Energy

Heavy Ion Sudy, Berkeley, May 18,1981

W.Nitsche et al

Pre-Equilibrium Process of Neutron Emission for Light Ion Induced Reactions neutron spectra

precompoundnucleus emission

stripping

knock-out reactions

two-nucleon transfer reactions, breakup reactions

two-nucleon

transfer reactions, fast neutrons

inner-shell ionization, ionatom collisions, $x$-ray spectra

knock-out reactions

precompoundnucleus emission, neutron emission 2nd French-Japan

Colloquium on

Heavy Ion Nuclear

Physics, Saclay,Fr

Oct 10, 1979,p.327

H.Ejiri 
$27 \mathrm{Al}$

${ }^{59} \mathrm{Co}$

162,164 Dy

$40,44 \mathrm{Ca}$

$93 \mathrm{Nb}$

93Nb

$150 \mathrm{Nd}$

$158,160 \mathrm{Gd}$

$\mathrm{Pb}$

$27 \mathrm{Al}$

197 Au

$58 \mathrm{Ni}$
Spectra of the Protons, Deuterons, and Tritons Produced by $50 \mathrm{MeV}$ Alpha Particles from $27 \mathrm{Al}$ and ${ }^{59} \mathrm{Co}$

Àngular Momentum Transfers

in Equilibrium and Pre-

Equilibrium Dy(alpha,xn gamma)

Reactions

Nuclear Fragmentation with $100 \mathrm{MeV}$ Alpha Particles

Spallation Cross Sections in the System $93 \mathrm{Nb}+{ }^{4} \mathrm{He}$

Excitation Functions and Isomer Ratios in the Reactions $93 \mathrm{Nb}$ (alpha, $\mathrm{xn})(\mathrm{x}=1-4)$

Calculation of Gamma-Ray Spectra Following AlphaInduced Fusion Reactions

Pre-Compound Decay into Cumplex Particle Channels

Influence of Radiation Damage on Perturbed Angular

Distributions

Mechanism of (alpha,p), (alpha,d), and (alpha,t) Reactions in $27 \mathrm{Al}, 59 \mathrm{Co}$, and $197 \mathrm{Au}$

Multiplicity of the Gamma Cascades in the 61 Cu Continuum; Dependence on Excitation Energy of the Entry States
Bull Acad Sci USSR

Phys Series

39,138(1975)

A.D.Duisebaev et al

J Phys Soc Japan

46,1025(1979)

Y Nagai et al

Zeit fur Physik

A292,35(1979)

H.Loehnet et al

$\mathrm{J}$ Inorg \& Nucl

Chem 41,791 (1979)

C.L.Branquinho

et al

$\mathrm{J}$ Inorg \& Nucl

Chem 41,617(1979)

C.L.Branquinho

et al

Nucl Phys A315, 169

(1979) T.Koeling

AIP (Amer Inst of

Physics) Conf Proc.

47,660(1978)

H.Machner

Phys Rev C23,90

(1981) E.Dafni

et al

Sov J Nucl Phys

27,613(1978)

A.D.Duisebaev

et al

Phys Rev C18,119 (1978) J.H.Barker

et al ganima spectra

energy spectra

precompound nucleus emission, gamma radiation

spallation

spallation, precompoundnucleus emission

precompoundnucleus emission

inelastic scattering, precompoundnucleus emission

radiation effects, neutron spectra

stripping, precompoundnucleus emission

gamma cascades 
166 $\mathrm{Er}$

150Nd

Angular-Momentum Effects in

Pre-Equilibrium Processes

Exciton Model Approach to the Analysis of Alpha-ParticleInduced Nuclear Reactions

$209 \mathrm{Bi}$

$197 \mathrm{Au}$

$119 \mathrm{Sn}$

$\mathrm{Ni}$

${ }^{59} \mathrm{Co}$

$58 \mathrm{Ni}$

197 Au

$56 \mathrm{Fe}$

$58 \mathrm{Ni}$
Total Reaction Cross Section

from the Difference of the

Rutherford and Measured

Elastic Cross Sections

Unified Model of Preequilibrium Decay

Quasi-Free Scattering Model

for Reactions Involving

Clusters

$X$ ? ay Prixduction by 5.2-27

Me $Y$ : Ipha Particles

Total Reaction Cross Sections from Heavy Ion Elastic

Scattering

Continuous Spectra of Protons

Emitted in Alpha-Particle

Induced Reactions

Spectra of Singly Charged

Particles Emitted in the

Bombardment of Intermediate

and Heavy Nuclei by $50.5 \mathrm{MeV}$

Alpha Particles
Phys Rev C17,601

(1978) D.G.Saran-

tites et al

Roczniki Chemii

(Poland) 6, 1(1976)

E.Gadioli et al

Phys Rev C16,1767

(1977) H.Wojciec-

howski et al

Zeit fur Physik

A281, 129(1977)

J.Emst, J.Rama Rao

Nucl Phys A273,125

(1976) A.Mignerey

et al

X-Ray Spectrometry

(England) 6,90

(1977) T.Badica

et al

Phys Lett B63,413

(1976) H.Wojciec-

howski et al

Nucl Phys A265,376

(1976) A.Alevra

et al

Bull Acad Sci USSR

Phys Series

40,152(1976)

N.T.Burtebaev et al precompoundnucleus emission

composite models, stripping

Rutherford scattering, optical models

precompoundnucleus emission, unified model

quasi-free reactions, cluster model

x-ray emission, ionization

total cross

sections,

optical models

precompoundnucleus emission, stripping

energy spectra 
$41 \mathrm{~K}$

${ }^{116} \mathrm{Sn}$

$94,96 \mathrm{Zr}$

$89 \mathrm{Y}$

$27 \mathrm{Al}$

51V

${ }^{109} \mathrm{Ag}$

$40,42 \mathrm{Ca}$

${ }^{44} \mathrm{Ca}$

$12 \mathrm{C}, 6 \mathrm{Li}$

${ }^{14} \mathrm{~N}, 124 \mathrm{Sn}$

${ }^{209} \mathrm{Bi}$

${ }^{209} \mathrm{Bi}, \mathrm{Al}$

$\mathrm{Mg}$

6Li

$\mathrm{Fe}, \mathrm{Ta}$

158Dy

$164 \mathrm{Er}$

${ }^{174} \mathrm{Hf}$

$168 \mathrm{Yb}$

$63,65 \mathrm{Cu}$
Analysis of the $41 \mathrm{~K}$ (alpha,n)

Reaction in the Pre-Equili-

brium Model of Nuclear

Reactions

Scattering of $65-\mathrm{MeV}$ Alpha

Particles from $89 \mathrm{Y}, 92 \mathrm{Zr}$,

$94 \mathrm{Zr}, 96 \mathrm{Zr}$ and $116 \mathrm{Sn}$

Reactions of $51 \mathrm{~V}$ and $27 \mathrm{Al}$ with 7 to $120 \mathrm{MeV}$ Alpha-

Particles (Equilibrium and

Non-Equilibrium Statistical

Analyses)

Theory of $(a, x n)$ Reactions

Scattering of Medium-Energy

Alpha Particles II. Micro-

scopic Analysis of Elastic

Scattering

Elastic Scattering of $104 \mathrm{MeV}$

Alpha Particles

Measurement of the K-Shell

Ionization Cross Sections

of $\mathrm{Al}$ and $\mathrm{Mg}$ for 1- to

5-MeV Alpha Particles

Tests of the Peripheral Model

for a Cluster Knockout

Reaction

Shielding of Neutrons from

80-MeV Alpha-Particle

Bombardment of Tantalum

Studies of Photon Cascades in (alpha,2n) and (alpha,3n)

Reactions on Rare-Earth

Nisclei

Differential Range Study of (alpha, xn) Reactions of

Copper
Acta Physica

Slovaca 25, 264(1975)

E.Betak

stripping

Phys Rev 180,1197

(1969) C.R.Bingham

et al

Nucl Phys A131,513

(1969) W.W.Bowman,

M.Blann

elastic and

inelastic

scattering

excitation

functions,

recoil ranges

Phys Rev 178,1688

(1969) D.Sperber

Phys Rev 178,1626

(1969) D.F.Jackson,

V.K.Kembhavi

Karlsruhe report

KFK-871(1968)

G.Hauser et al

Phys Rev 182,9

(1969) B.Sellers

et al

Phys Rev Lett 22,

408(1969)

H.B.Pugh et al

Nucl Sci Eng 35,220

(1969) W.W.Wadman III

Nucl Phys A124,401

(1969) C.D.Kavaloski

et al

cluster knockout

gamma radiation

Phys Rev 176, 1166

(1968) N.T.Porile,

I.Fujiwara fast neutrons

$\mathrm{x}$-radiation, ionization

excitation

functions,

(alpha,n),

(alpha,2n)

microscopic

analysis using

optical potential

elastic

scattering

(alpha,2n) and

(alpha,3n),

recoil ranges 
Table IV (Cont.)

$51 \mathrm{~V}$

${ }^{27} \mathrm{Al}$

$\mathrm{Pb}, \mathrm{Au}$

$169 \mathrm{Tm}$

$165 \mathrm{Ho}$

$160,12 \mathrm{C}$

$14 \mathrm{~N}$

$\mathrm{N}, \mathrm{C}$

Ta

$238 U$

$232 \mathrm{Th}$

$27 \mathrm{Al}$
Excitation Functions, Recoil

Ion Ranges, Equilibrium, and

Nonequilibrium Statistical

Analysis of Reactions Induced

in $27 \mathrm{Al}$ and $51 \mathrm{~V}$ by 7 to 120

$\mathrm{MeV}{ }^{4} \mathrm{He}$ Ions

The Evaporation of Alpha-

Particles from Heavy Nuclei

Experimental Study and Analy-

sis of the Excitation

Functions $165 \mathrm{Ho}(\mathrm{alpha}, \mathrm{xn})$ and

$169 \mathrm{Tm}$ (alpha,xn)

Cross Sections of Reactions

Induced by $90-\mathrm{MeV}$ Alpha

Particles on Carbon, Nitrogen

and Oxygen Nuclei

Formation Cross Sections of

Fragments with Mass Greater

Than or Equal to 6 Emitted

in Reactions Induced by Alpha

Particles of $90 \mathrm{MeV}$ in $\mathrm{C}$ and

$\mathrm{N}$ Targets

Shielding Attenuation of

Neutrons from 80-MeV Alpha-

Particle Bombardment of

Elemental Tantalum

$12 \mathrm{C}, 160$

$10 \mathrm{~B}, 27 \mathrm{Al}$

Quasi-Elastic (alpha,2 alpha)

Reaction at $700 \mathrm{MeV}$ on Several

Light and Medium Nuclei

Fragment Angular Distributions from Fission of $238 \mathrm{U}, 209 \mathrm{Bi}$, and ${ }^{197} \mathrm{Au}$ by $140-\mathrm{MeV} 4 \mathrm{He}$ Ions

Gamma-Ray Production Cross-

Sections from the Interaction

of $140 \mathrm{MeV}$ Alpha-Particles

with $232 \mathrm{Th}$

Gamma-Ray Production Cross-

Sections from the Interaction

of $140 \mathrm{MeV}$ Alpha-Particles

with $27 \mathrm{Al}$ (II)
No university in-

formation given

Thesis (1968)

W.W.Bowman

Nucl Phys A122,481

(1968) G.Chenevert

et al

Nucl Phys A121,131

(1968) J.Sau et al

Compt Rend Ser A \&

B 266,963(1968)

C. Jacquot et al

Compt Rend Ser

A \& B 266,815

(1968) M.Jung

et al

Lawrence Berkeley

report UCRL-16359

(ND) W.W.Wadman

College of Wm \&

Mary,Williamsburg,

Va, Thesis(1975)

W.E.Dollhopf

Zeit fur Phys

A274,131(1975)

R.G.Clark et al

Univ of Maryland report ORO-4856-26

pp.358,359(1975)

W.F.Hornyak et al

Univ of Maryland report ORO-4856-26

pp. 356-357(1975)

W.F.Hornyak,N.S.Wall excitation

functions and

recoil ion ranges

inelastic

scattering,

evaporation

model analysis

excitation

functions,

(alpha,xn)

$82-98 \mathrm{MeV}$ alpha

particles

production of light nuclei

energy losses

quasi-elastic scattering, recoils

fission fragments

gamma radiation

gamma radiation 
$27 \mathrm{Al}$

$\mathrm{Hg}, \mathrm{Ta}$

${ }^{209} \mathrm{Bi}$

${ }^{197} \mathrm{Au}$

$238 \mathrm{U}, 209 \mathrm{Bi}$

$197 \mathrm{Au}$

$\mathrm{Cu}$

Al, O

$\mathrm{C}, \mathrm{Be}$

$12 \mathrm{C}$

$27 \mathrm{Al}$

$12 \mathrm{C}$

$27 \mathrm{Al}$

$208 \mathrm{~Pb}$

$58 \mathrm{Ni}$

${ }^{9} \mathrm{Be}, 7 \mathrm{Li}$

$12 \mathrm{C}, 14 \mathrm{~N}$
Gamma-Ray Production Cross-

Sections from the Interaction of $140 \mathrm{MeV}$ Alpha-Particles with $27 \mathrm{Al} \mathrm{(I)}$

Search for Heavy Fast Fragments or Recoil Nuclei in the Interaction of 21-GeV Neutrons and $700-\mathrm{MeV}$ Alphas on $\mathrm{Hg}$, $\mathrm{Ta}$, $\mathrm{Ce}, \mathrm{Y}, \mathrm{Cu}, \mathrm{Mn}$, and $\mathrm{Ca}$

Linear Momentum Transfer in Reactions Between 140-MeV $4 \mathrm{He}$ Ions and Heavy Nuclei

Fission of $238 \mathrm{U},{ }^{209} \mathrm{Bi}$, and $197 \mathrm{Au}$ with Intermidiate-

Energy $4 \mathrm{He}$ Ions

Spallation of Copper by HighEnergy Alpha Particles

Alpha-Spallation Cross Sections at $920 \mathrm{MeV}(230 \mathrm{MeV} / \mathrm{N})$ in $27 \mathrm{Al}, 16 \mathrm{O}, 12 \mathrm{C}$, and ${ }^{9} \mathrm{Be}$, and Application to CosmicRay Transport

Calculated Secondary-Particle Spectra from Alpha-Particleand Carbon-Induced Nuclear Reactions

Calculated Secondary-Particle Spectra from Alpha-Particleand Carton-Induced Nuclear Reactions

Scattering of 139-MeV Alpha Particles by $58 \mathrm{Ni}$ and $208 \mathrm{~Pb}$

Experimental and Semi-Empirical Study of the Spallation of Light Nuclei. Application to the Nucleosynthesis of $\mathrm{Li}$, $B e$, and $B$ in the Galaxy
Univ of Maryland

gamma radiation

report ORO-4856-26

pp.354-355(1975)

W.F.Homyak et al

Helv Phys Acta 48 ,

p. 343 (1975)

H.W Reist et al

recoils

Phys Rev C10,2416

(1974) V.E.Viola

et al

3. Symp on the

Phys \& Chem of

Fission, Aug 13, 1973 Rochester NY, V.E.Viola et al

Phys Rev C10, 150 (1974) P.J.Karol

Phys Rev C9, 1718 (1974) J.R.Radin

et al

Nucl Sci Eng 53,323 (1974) T.A.Gabriel et al

Oak Ridge report

ORNL-TM-4334(1973)

T.A.Gabriel et al

Phys Rev C7,1938

(1973) D.A.Goldberg

et al

Univ of Paris, Orsay Thesis, FRNC-TH-333
(1972) M.Rey CampagnolleEpherre optical model, elastic and inelastic scattering spallation

fission, linear momentum transfer

nuclear reaction kinetics

spallation

spallation

spallation

neutron spectra 
C

${ }^{9} \mathrm{Be}, 12 \mathrm{C}$

$160,27 \mathrm{Al}$

$58 \mathrm{Ni}, 65 \mathrm{Cu}$

$197 \mathrm{AU}$

Ag, In,La

Pr,Ho

$40 \mathrm{Ar},{ }^{9} \mathrm{Be}$

${ }^{209} \mathrm{Bi}$

$40 \mathrm{Ca}$

$208 \mathrm{~Pb}, 6 \mathrm{Li}$

$14 \mathrm{~N}, 16 \mathrm{O}$

$124 \mathrm{Sih}_{\mathrm{i}} 90 \mathrm{Zr}$

$12 \mathrm{C} .27 \mathrm{Al}$

$93 \mathrm{Nb}$

$\mathrm{C}, \mathrm{Pb}$

$\mathrm{Si}, \mathrm{Sn}$

$12 \mathrm{C}, \mathrm{Pb}$

$28 \mathrm{Si}$

$\mathrm{C}, \mathrm{Ne}, \mathrm{Ni}$

$27 \mathrm{Al}$

$93 \mathrm{Nb}$
Scattering of $139 \mathrm{MeV}$ Alpha

Particles by $12 \mathrm{C}$

Alpha-Sfallation Cross Sections at $920 \mathrm{MeV}(230 \mathrm{MeV} / \mathrm{N})$ in $27 \mathrm{Al}, 160,12 \mathrm{C}$, and $9 \mathrm{Be}$, and Application to Cosmic Ray Transport

Study of (alpha, alpha n) Reaction with $90-\mathrm{MeV}$ Helium Ions

Fission of Medium Mass Nuclei Induced by $167 \mathrm{MeV}$ Alpha Particles

Measured Differential Cross Sections for Elastic and Inelastic Scattering of $104-\mathrm{MeV}$ Aipha Particles

Approximate High-Energy AlphaParticle Nucleus Collision Model

Elastic Scattering of $166 \mathrm{MeV}$ Alpha Particles

166-MeV Elastic and Inelastic Alpha-Particle Scattering: Macroscopic and Microscopic Analysis

Inelastic Scattering of 104MeV Alpha-Particles on $12 \mathrm{C}$, $20 \mathrm{Ne}$, and $64 \mathrm{Ni}$

Interaction of High Energy Alpha Particles with Nuclei
J Phys (Paris) Col$\log 5,150$ (1972)

G.Tibell et al

No university information given Thesis(1972) J.R.Radin Univ. microfilm order $\# 72-21,534$

Phys Rev C6, 1293 (1972) L.B.Church

Nucl Phys A173,520 (1971) B.D.Pate, J.Peter

Kar:sruhe report NP-18920(1970)

DHabs et al

Nucl Sci Eng 44, 104 (1971) T.A.Gabriel et al

J Phys (Paris)

Coll. 31 Suppl

$5 \& 6$ C2.85(1970)

I.Brissaud,B. Tatischeff

Nucl Phys A155,89

(1970)

B.Tatischeff,

I.Brissaud

Nucl Phys A143,373

(1970) J.Specht

et al

Yadern Fiz 11,36

(1970) A.I.Vikhrov

et al stripping, elastic and inelastic scattering, optical models

spallation

knock-out reactions

fission products, particle tracks

elastic, inelastic scattering

neutron and proton spectra, excitation functions

optical-model analysis, elastic scattering

optical potentials, elastic and inelastic scattering

inelastic scattering nuclear cascades 


\begin{tabular}{|c|c|c|c|}
\hline \multirow[t]{2}{*}{$\begin{array}{l}28 \mathrm{Si}, \mathrm{Pb} \\
\mathrm{Sn}, 12 \mathrm{C}\end{array}$} & $\begin{array}{l}\text { Elastic Scantering of } 166 \mathrm{MeV} \\
\text { Alpha Particles on D, C, Si, } \\
\text { Sn, and Pb }\end{array}$ & $\begin{array}{l}\text { Phys Lett B30,324 } \\
\text { (1969) I.Brissaud } \\
\text { et al }\end{array}$ & $\begin{array}{c}\text { elastic scattering } \\
.\end{array}$ \\
\hline & $\begin{array}{l}\text { Thermoluminescence of Fused } \\
\text { Quartz Irratiated with Charged } \\
\text { Particles }\end{array}$ & $\begin{array}{l}\text { Opt Spectrosc 39, } \\
\text { 564(USSR)(1975) } \\
\text { R.I.Shekhmametev, } \\
\text { G.Por }\end{array}$ & $\begin{array}{l}\text { physical radiation } \\
\text { effects, } \\
\text { thermoluminescenc }\end{array}$ \\
\hline $27 \mathrm{Al}$ & $\begin{array}{l}\text { Spectra vi } \mathrm{f}, \mathrm{d} \text {, and } \mathrm{t} \text { During } \\
\text { Bombardmen: of } 27 \mathrm{Al} \text { and } 59 \mathrm{Co} \\
\text { Nuciei with } 50.5-\mathrm{MeV} \text { Alpha } \\
\text { Particles }\end{array}$ & $\begin{array}{l}\text { Izv Akad Nauk Kaz } \\
\text { SSR Ser Fiz Mat 4, } \\
\text { 19(1974) } \\
\text { A.D.Duisebaev et al }\end{array}$ & transfer reactions \\
\hline $\begin{array}{l}197 \mathrm{Au} \\
\mathrm{Tl}, \mathrm{Hg}\end{array}$ & $\begin{array}{l}\text { Investigation of Multinucleon } \\
\text { Emission Induced by Alpha- } \\
\text { Particles on } 197 \mathrm{Au} \text { in the } \\
\text { Energy Range } 20 \text { to } 170 \mathrm{MeV}\end{array}$ & $\begin{array}{l}\text { Nucl Phys A250, } 149 \\
\text { (1975) A.Djaloeis } \\
\text { et al }\end{array}$ & $\begin{array}{l}\text { multi-nucleon } \\
\text { transfer reactions }\end{array}$ \\
\hline \multirow[t]{2}{*}{$40 \mathrm{Ca}$} & $\begin{array}{l}\text { Microscopic Analysis of Alpha- } \\
\text { Nucleus Scattering in the } \\
\text { Energy Range from } 20 \text { to } \\
140 \mathrm{MeV}\end{array}$ & $\begin{array}{l}\text { Univ of Colorado } \\
\text { report COO-535-733 } \\
\text { p.55(1975) B.W.Ridley, } \\
\text { H.H.Chang }\end{array}$ & optical models \\
\hline & $\begin{array}{l}\text { Fission of Pre-Actinide Nuclei } \\
\text { Excitation Functions for the } \\
\text { (alpha,f) Reaction }\end{array}$ & $\begin{array}{l}\text { Yad Fiz } 21,1185 \\
\text { (1975) A.V.Ignat- } \\
\text { yuk et al }\end{array}$ & $\begin{array}{l}29 \text { nuclei from } \\
\mathrm{Hf} \text { to } \mathrm{Bi}\end{array}$ \\
\hline $168 w$ & $\begin{array}{l}\text { On the Careful Determination } \\
\text { of Gamma-Ray Angular Distri- } \\
\text { butions in (alpha,n gamma) } \\
\text { Reactions: Example of the } 186 \mathrm{w} \\
\text { (aipha,7n gamma)185Os Reaction }\end{array}$ & $\begin{array}{l}\text { Nucl Instr Meth } \\
\text { 125,53(1975) } \\
\text { H.M.Jaeger et al }\end{array}$ & gamma spectra \\
\hline $238 \mathrm{U}$ & $\begin{array}{l}\text { Linear Momentum Transfier in } \\
\text { Reactions Between } 140-\mathrm{MeV} 4 \mathrm{He} \\
\text { Ions and Heavy Nuclei }\end{array}$ & $\begin{array}{l}\text { Univ of Maryland } \\
\text { report ORO-4028-0021 } \\
\text { p.53(1974) V.E.Viola } \\
\text { et al }\end{array}$ & fission \\
\hline $203,205 \mathrm{Tl}$ & $\begin{array}{l}\text { Helium Ion-Induced Fission of } \\
203 \mathrm{Tl} \text { and } 205 \mathrm{Tl} \text { at } 31,42 \text {, and } \\
65 \mathrm{MeV}\end{array}$ & $\begin{array}{l}\text { J Inorg Nucl Chem } \\
\text { 36,3643(1974) } \\
\text { R.D.Kieburtz, E.F.Neuzil }\end{array}$ & $\begin{array}{l}\text { total fission } \\
\text { cross section }\end{array}$ \\
\hline $44 \mathrm{Sc}$ & $\begin{array}{l}\text { Angular Momentum Effects in } \\
\text { the Pre-Equilibrium Staris- } \\
\text { tical Model }\end{array}$ & $\begin{array}{l}\text { Acta Phys Slovaca } \\
\text { 24,103(1974) } \\
\text { P.Oblozinsky, } \\
\text { I.Ribansky }\end{array}$ & $\begin{array}{l}\text { excitation } \\
\text { functions, } \\
\text { neutron spectra }\end{array}$ \\
\hline $209 \mathrm{Bi}$ & $\begin{array}{l}\text { Excitation Functions of Reac- } \\
\text { tions of Alpha Particles with } \\
\text { Energies Between } 40 \text { and } 100 \\
\text { MeV on } 209 \mathrm{Bi}\end{array}$ & 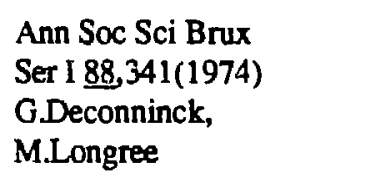 & $\begin{array}{l}\text { excitation } \\
\text { functions }\end{array}$ \\
\hline
\end{tabular}




\begin{tabular}{|c|c|c|c|}
\hline $58,60,62 \mathrm{Ni}$ & $\begin{array}{l}\text { Elastic Scattering of } 60-\mathrm{MeV} \\
\text { Alpha Particles by the Even } \\
\text { Nickel Isotopes }\end{array}$ & $\begin{array}{l}\text { Phys Rev C9,1002 } \\
\text { (1974) D.G.Madland } \\
\text { et al }\end{array}$ & $\begin{array}{l}\text { optical-model } \\
\text { analysis }\end{array}$ \\
\hline $\begin{array}{l}232 \mathrm{Th} \\
238 \mathrm{U}\end{array}$ & $\begin{array}{l}\text { Range-Energy Relation for Low } \\
\text { Velocity Heavy Ions in a Plas- } \\
\text { tic Detector. Applications to } \\
\text { the Study of the Fission of } \\
\text { Uranium, Thorium, Bismuth, } \\
\text { Lead, and Gold Induced by Alpha } \\
\text { Particles having Energies } \\
\text { Between } 58 \text { and } 100 \mathrm{MeV}\end{array}$ & $\begin{array}{l}\text { Univ of Strasbourg } \\
\text { Thesis, FRNC-TH- } \\
\text { 466(1972) } \\
\text { J.Ralarosy }\end{array}$ & $\begin{array}{l}\text { dielectric track } \\
\text { detectors, } \\
\text { stopping power }\end{array}$ \\
\hline $\begin{array}{l}197 \mathrm{Au} \\
232 \mathrm{Th} \\
238 \mathrm{U}\end{array}$ & $\begin{array}{l}\text { Fission Cross Sections of } \\
\text { Uranium, Thorium, Bismuth, } \\
\text { Lead, and Gold Induced by 58- } \\
\text { to } 100-\mathrm{MeV} \text { Alpha Particles }\end{array}$ & $\begin{array}{l}\text { Phys Rev C8, } 2372 \\
\text { (1973) ?.Ralarosy } \\
\text { et al }\end{array}$ & fission \\
\hline \multirow[t]{2}{*}{$\begin{array}{l}\mathrm{Co}_{1} 197 \mathrm{Au} \\
181 \mathrm{Ta} \\
184 \mathrm{~W}\end{array}$} & $\begin{array}{l}\text { Proton Spectra from 54.8-MeV } \\
\text { Alpha-Particle Reactions: } \\
\text { Precompound Emission }\end{array}$ & $\begin{array}{l}\text { Fhys Rev C.8,2155 } \\
\text { (1973) A.Chevarier } \\
\text { et al }\end{array}$ & proton spectra \\
\hline & $\begin{array}{l}\text { Excitation Functions and } \\
\text { Photon Emission in Compound- } \\
\text { Nucleus Reactions with High } \\
\text { Angular Momentum }\end{array}$ & $\begin{array}{l}\text { Phys Rev C7,1973 } \\
\text { (1973) J.Gilat } \\
\text { et al }\end{array}$ & $\begin{array}{l}\text { gamma cascades, } \\
\text { emission }\end{array}$ \\
\hline $\begin{array}{l}57 \mathrm{Fe}, 60 \mathrm{Ni} \\
118 \mathrm{Sb}\end{array}$ & $\begin{array}{l}\text { Precompound Processes in } \\
\text { (alpha,n) Reactions }\end{array}$ & $\begin{array}{l}\text { Univ of Lyon report } \\
\text { LYCEN-7303(1973) } \\
\text { A.Alevra et al }\end{array}$ & $\begin{array}{l}\text { pre-equilibrium } \\
\text { and hybrid models }\end{array}$ \\
\hline $\begin{array}{l}56 \mathrm{Fe}, 103 \mathrm{Rh} \\
116 \mathrm{Sn}\end{array}$ & $\begin{array}{l}\text { Precompound Processes in } \\
\text { (alpha,n) Reactions }\end{array}$ & $\begin{array}{l}\text { Nucl Phys A209,557 } \\
\text { (1973) A.Alevra } \\
\text { et al }\end{array}$ & neutron spectra \\
\hline $197 \mathrm{Au}$ & $\begin{array}{l}\text { Pre-Equilibrium Decay and the } \\
\text { Exciton Model }\end{array}$ & $\begin{array}{l}\text { Nucl Phys A205,545 } \\
\text { (1973) I.Ribansky } \\
\text { et al }\end{array}$ & excitons \\
\hline \multirow[t]{2}{*}{$\begin{array}{l}{ }^{59} \mathrm{Co}, \mathrm{Cu} \\
\mathrm{Ag}, \mathrm{W}\end{array}$} & $\begin{array}{l}\text { Proton Emission Spectra in } \\
\text { Alpha Induced Nuclear Reac- } \\
\text { tions at } 54.8 \mathrm{MeV} \text {. De- } \\
\text { Excitation Before Statistical } \\
\text { Equilibrium is Reached }\end{array}$ & $\begin{array}{l}\text { Thesis, no univer- } \\
\text { sity given, LYCEN- } \\
7241 \text { (1972) M.D.Tran }\end{array}$ & energy spectra \\
\hline & $\begin{array}{l}\text { Inelastic Interactions of High } \\
\text { Energy Helium Ions with Atomic } \\
\text { Nuclei }\end{array}$ & $\begin{array}{l}\text { Yadern Fiz } 17,434 \\
\text { (1973) V.S.Bara- } \\
\text { shenkov et al }\end{array}$ & $\begin{array}{l}\text { intranuclear } \\
\text { cascade model, } \\
\text { nuclear cascades }\end{array}$ \\
\hline
\end{tabular}




$\begin{array}{ll}\text { Si } & \text { EPR in Single Crystals of } \\ \text { Silicon Irradiated by Alpha- } \\ \text { - idrticles }\end{array}$

${ }^{58} \mathrm{Ni} \quad$ Optical Potential for Nuclei with Collective States

${ }^{59} \mathrm{Co},{ }^{63} \mathrm{Cu}$

$197 \mathrm{Au}$

$165 \mathrm{Ho}$

$\mathrm{K}$

$40 \mathrm{Ca}$

lithium

floride

$232 \mathrm{Th}$

$235,238 \mathrm{U}$

$233 \mathrm{U}$

$181 \mathrm{Ta}$

${ }^{209} \mathrm{Bi}$
Inelastic Scattering in the

Continuum. I. DWBA Analysis

of Angular Distributions

Precompound Emission Induced by Protons and Alpha Particles of about 50-MeV Excitat' sit

Calculations of the Excitation Functions and Isomer Ratios of the 41K(alpha, $n$ ) 44 Sc Reaction in the Equilibrium and PreEquilibrium Statistical Models

Energy Dependence of Elastic Alpha-Particle Scattering: Micrus;upic Model

Coloration of $\mathrm{LiF}$ by $56-\mathrm{MeV}$ Alpha Particles and 28-MeV Deuterons II. $\mathrm{F}_{2}+$ Centre Evolution

22 Dependence of Stopping Cross Sections for Low-Energy Alpha Particles

Energy Dependence of Average Kinetic Energy of Fragments from Alpha-Induced Fission of $232 \mathrm{Th}, 235 \mathrm{U}$, and $236 \mathrm{U}$

Mass and Energy Distributions from 77.3-MeV.4 $\mathrm{He}$-Induced Fission of $181 \mathrm{Ta}, 209 \mathrm{Bi}$, and 233U: A Test of Liquid-DropModel Predictions
Izv Akad Nauk Kaz SSR Ser Fiz Mat 4 . 39(1972) Yu.V.Gorelkinskii et al

Lett Nuovo Cim 6 , 277(1973)

E.Kujawski

Univ of Lyon report LYCEN-7263(1972)

A.Demeyer et al

Univ of Lyon report LYCEN-7252(1972

A.Chevarier et al

Nucl Phys A195,269

(1972) P.Oblozinsky, I.Ribansky

Phys Rev C4,1254

(1972) G.M.Lerner et al

Phys Status Solidi

(A) $\underline{10}, 67(1972)$

P.Thevenard et al

Phys Lett A38,267 (1972) W.K.Chu, D.Powers

Yadem Fiz 14,514 (1971) V.N.Okolovich, A.F.Pavlov

Phys Rev C5,528

(1972) F.Plasil,

H.W.Schmitt crystal defects, fast neutrons

elastic scattering, optical models

inelastic scattering, angular distributions and spectra

heavy nuclei, intermediate mass nuclei, precompound emission

excitation functions, pre-equilibrium statistical models

39.6 to 115.4

$\mathrm{MeV}$, microscopic optical model

radioinduced color centers

stopping cross sections

fission

fission 
light

nuclei

${ }^{209} \mathrm{Bi}, 238 \mathrm{U}$

206,208Pb

232 Th

$238 \mathrm{U}$

$24 \mathrm{Mg}$

$28 \mathrm{Si}, 12 \mathrm{C}$

$63,65 \mathrm{Cu}$
Identification of Medium-

Energy Alpha-Particle-Induced

Reactions

Calculation of Cross Section

for Compound-Nucleus Formation

by Means of Generalized S-

Matrix Parameters

Josephson Current Flow in Pure Superconducting/Normal/

Superconduction Junctions

Mechanism of Nuclear Reactions Induced by High Energy Alpha Particles

Study of Quasi-Elastic Scattering Experiments on Light Nuclei

Fission of $232 \mathrm{Th}$ Induced by Intermediate-Energy $4 \mathrm{He}$ Ions

Direct Interaction Effects in the Fission of $238 \mathrm{U}$ with 63.5 $\mathrm{MeV} 4 \mathrm{He}$ Ions

Heavy Ion Emission from Light Nuclei

\footnotetext{
Excitation Functions of Reactions Induced by Alpha Particles with Maximum Energy of $38 \mathrm{MeV}$ on Copper Isotopes

X-Ray Production by AlphaParticle Impact
}

Phys Rev C5,579

(1972) MJung et al

nuclear emulsions

Izv Akad Nauk Kaz

s-matrix

SSR Ser Fiz Mat

2,62(1971) V.Yu.Conchar,

K.S.Zheltonog

Phys Rev B5,72(1972)

W.D.Loveland,

Y.S.Shum

J Phys (Paris) 32 ,

243(1971) R.Bimbot,

Y.Beyec

Univ of Lyon Thesis

LYCEN-7134(1971)

M.Chevallier

Phys Rev C4,1361

(1971) T.C.Roginski

et al

Nucl Phys A174,321

(1971) V.E.Viola

et al

Thesis(1967) no

university given,

Univ. microfilms

order \#71-16,994

C.R.Rudy Jr.

Izv Akad Nauk Kaz

SSR Ser F 2 Mat

4,1(1970)

O.A.Zhukova et al

Phys Rev A4,955

(1971) J.D.Garcia fission

knock-out reactions

impulse

approximation, scattering, light nuclei

fission at 20

to $41 \mathrm{MeV}$

angular

correlation

angular

distribution excitation

functions,

impulse approximation, ionization, $x$ radiation gamma spectra 
$93 \mathrm{Nb}$

$54 \mathrm{Fe}$

$160,12 \mathrm{C}$

$197 \mathrm{Au}$

${ }^{169} \mathrm{Tm}$

165 Ho

Ni,Pd,Ag

$\mathrm{Sn}, \mathrm{Tm}, \mathrm{Cu}$

$\mathrm{Zr}, \mathrm{Nb}, \mathrm{Fe}$

Rh,Au

C.N

$12 \mathrm{C}$

$14 \mathrm{~N}$
Hybrid Model for Pre-Equilibrium Decay in Nuclear Reactions

Study of the Fission and Fragmentation of Heavy Nuclei by High Energy Projectiles

Hydrogen and Helium Particles Produced by $59-\mathrm{MeV}$ Alpha Particle Bombardment of $12 \mathrm{C}$, 160 , and ${ }^{54} \mathrm{Fe}$

Measurement and Equilibrium Statistical-Model Calculation of Excitation Functions of the $197 \mathrm{Au}($ alpha, xn) Reactions in the Energy Range from 16 to $103 \mathrm{MeV}$

Analysis of Inelastic Scattering of Alpha Particles in Region $Z=12$ to 40

Application of the Statistical Model to the Study of (alpha, $x$ ) Excitation Functions of $\mathrm{Ho}$ and $\mathrm{Tm}$

$X$-Ray Emission Induced by 30 to 80-MeV Alpha Particles

Production Cross Section of Isotopes Emitted in 70-MeV Alpha-Particle Interactions on $C$ and $N$ Targets

Total Experimental Cross Sections of Nuclear Reactions Produced by $90 \mathrm{MeV}$ and $70 \mathrm{MeV}$ Alpha Particles on $12 \mathrm{C}$ and $14 \mathrm{~N}$ Target. Some Nucleosynthesis and Nuclear Spectroscopy Applications
Phys Rev Lett 27. 337(1971) M.Blann

pre-equilibrium emission

heavy nuclei, fission fragments/ mass distributions

spectra ORNL-4694(1971) F.E.Bertrand, R.W.Peele

Nucl Phys A168,129 (1971) H.E.Kurz et al

excitation functions
Izv Akad Nauk Kaz SSR Ser Fiz Mat 6,3(1969)

V.Yu.Gonchar et al

J Phys (Paris) Colloq 31,5(1970)

A.Demeyer et al

Nucl Phys A154,561 (1970) R.L.Watson et al

Phys Rev C2,1194 (1970) C.BaixerasAiguabella et al

Int Conf Nucl Reactions Induced by Heavy Ions, Heidelberg, Ger. p.701(1970), C.Jacquot et al inelastic scattering, $A=24$ to 140 nuclei

(alpha,xn) up to $150 \mathrm{MeV}$

x-radiation 


\begin{tabular}{|c|c|c|c|}
\hline $197 \mathrm{Au}$ & $\begin{array}{l}\text { Experimental Determination } \\
\text { and Statistical-Model Analy- } \\
\text { ses of Excitation Functions } \\
\text { and Recoil Ion Ranges from } \\
\text { Reactions Induced in } 197 \mathrm{Au} \text { by } \\
\text { 19- to } 100 \mathrm{MeV} 4 \mathrm{He} \text { Ions }\end{array}$ & $\begin{array}{l}\text { no university } \\
\text { information, } \\
\text { Thesis(1969) } \\
\text { F.M.Lanzafame }\end{array}$ & $\begin{array}{l}\text { excitation } \\
\text { function }\end{array}$ \\
\hline $204 \mathrm{~Pb}$ & $\begin{array}{l}\text { Helium Ion-Induced Fission of } \\
204 \mathrm{~Pb} \text { at } 65,41,37 \text {, and } 25 \\
\mathrm{MeV}\end{array}$ & $\begin{array}{l}\text { J Inorg Nucl Chem } \\
\text { 32,1761(1970) } \\
\text { W.K.Hensley, E.F.Neuzil }\end{array}$ & $\begin{array}{l}\text { fission } \\
\text { products }\end{array}$ \\
\hline $197 \mathrm{Au}$ & $\begin{array}{l}\text { Reactions of }{ }^{197} \text { Au with } 19- \\
\text { to } 100-\mathrm{MeV} 4 \mathrm{He} \text { Ions: Equili- } \\
\text { brium Statistical Model } \\
\text { Analyses }\end{array}$ & $\begin{array}{l}\text { Nucl Phys A142,545 } \\
\text { (1970) } \\
\text { F.M.Lanzafame, } \\
\text { M.Blann }\end{array}$ & $\begin{array}{l}\text { equilibrium } \\
\text { statistical model }\end{array}$ \\
\hline$C, N$ & $\begin{array}{l}\text { Cross Sections of Fragments } \\
\text { E-mitted in Spallation Reac- } \\
\text { tions of Carbon and Nitrogen } \\
\text { with } 90-\mathrm{MeV} \text { Alpha Particles }\end{array}$ & $\begin{array}{l}\text { Phys Rev } 188,1517 \\
\text { (1969) MJung et al }\end{array}$ & spallation \\
\hline $206 \mathrm{~Pb}$ & $\begin{array}{l}\text { Mechanism of Nuclear Reactions } \\
\text { Induced by High-Energy Alpha } \\
\text { Particles. I. Reactions } \\
206 \mathrm{~Pb}(\text { alpha,xn) } 2: 0-x \mathrm{Po}\end{array}$ & $\begin{array}{l}\text { J Phys (Paris) } 30 \\
\text { 513(1969) R.Bimbot } \\
\text { et al }\end{array}$ & $\begin{array}{l}\text { excitation } \\
\text { functions }\end{array}$ \\
\hline $\begin{array}{l}206,207 \mathrm{~Pb} \\
208 \mathrm{~Pb}\end{array}$ & $\begin{array}{l}\text { Alpha Particle Scattering on } \\
\mathrm{Pb} \text { Isotopes }\end{array}$ & $\begin{array}{l}\text { Yadem Fiz } 9,702 \\
\text { (1969) V.Yu.Gonchar } \\
\text { et al }\end{array}$ & elastic scattering \\
\hline $\begin{array}{l}94,96 \mathrm{Zr} \\
116 \mathrm{Sn}, 89 \mathrm{Y}\end{array}$ & $\begin{array}{l}\text { Scattering of } 65-\mathrm{MeV} \text { Alpha } \\
\text { Particles from } 89 \mathrm{Y}, 92 \mathrm{Zr} \text {, } \\
94 \mathrm{Zr}, 96 \mathrm{Zr} \text { and } 116 \mathrm{Sn}\end{array}$ & $\begin{array}{l}\text { Phys Rev } 180,11.37 \\
(1969) \text { C.R.Bingham } \\
\text { et al }\end{array}$ & $\begin{array}{l}\text { elastic and } \\
\text { inelastic } \\
\text { scattering }\end{array}$ \\
\hline \multirow[t]{2}{*}{$\begin{array}{l}27 \mathrm{Al} \\
51 \mathrm{~V}\end{array}$} & $\begin{array}{l}\text { Reactions of } 51 \mathrm{~V} \text { and } 27 \mathrm{Al} \text { with } \\
7 \text { to } 120 \mathrm{MeV} \text { Alpha-Particles } \\
\text { (Equilibrium and Non-Equili- } \\
\text { brium Statistical Analyses) }\end{array}$ & $\begin{array}{l}\text { Nucl Phys A131,513 } \\
\text { (1969) W.W.Bowman, } \\
\text { M.Blann }\end{array}$ & $\begin{array}{l}\text { excitation } \\
\text { function, } \\
\text { recoils }\end{array}$ \\
\hline & $\begin{array}{l}\text { Angular Distribution of } \\
\text { Neutrons Produced by } 40 \text {-and } \\
\text { 80-MeV Alpha-Particles on a } \\
\text { Thick Tantalum Target }\end{array}$ & $\begin{array}{l}\text { Health Physics } \\
\text { (England) } 11,659 \\
\text { (1965) W.W.Wadman }\end{array}$ & spectra \\
\hline $56 \mathrm{Fe}$ & $\begin{array}{l}\text { Reactions Induced in } 56 \mathrm{Fe} \\
\text { with } 20-68 \mathrm{MeV} \mathrm{He}^{2+} \text { Ions }\end{array}$ & $\begin{array}{l}\text { Nature 206.494 } \\
\text { (1965) A.Ewart, } \\
\text { M.Blann }\end{array}$ & $\begin{array}{l}\text { excitation } \\
\text { functions }\end{array}$ \\
\hline
\end{tabular}


$\mathrm{Ge}$

$\begin{array}{ll}\mathrm{Cu}, \mathrm{Ni}, \mathrm{Zn} & \text { Cross Sections for (alpha, } \mathrm{n} \text { ) } \\ & \text { Reactions for Medium-Weight } \\ & \text { Nuclei }\end{array}$

$\mathrm{Al}, \mathrm{Au}$

$\mathrm{Al}, \mathrm{Bi}, \mathrm{C}$

$\mathrm{Au}, \mathrm{Fe}, \mathrm{Pb}$

$\mathrm{Al}, \mathrm{Pb}, \mathrm{Zn}$

$\mathrm{Ag}, \mathrm{Ta}$

$\mathrm{V}, \mathrm{Zn}$

${ }^{12} \mathrm{C},{ }^{14} \mathrm{~N}$

$16 \mathrm{O}$
Effects of Alpha-Particle

Irradiation on the $\mathrm{X}$-Ray

Intensities Diffracted by

Germanium Crystals

The Effects of Linear Energy

Transfer in the Radiation-

Induced Polymerization of

Several Vinyl Compounds

Reactions for Medium-Weight

Angular and Energy Distribu tions of Neutrons Emitted in Bombardments with $42 \mathrm{MeV}$ Alpha Particles

Total-Reaction Cross-Section Measurements for Charged

Particles

Production of Tritium in Lead and Aluminum by High Energy Protons, Deuterons and AlphaParticles

Range Straggling of Protons, Alpha Particles, 12Carbon and $160 x y g e n$ Ions

Scattering of 43-MeV Alpha

Particles by Nuclei

Angular Distributions for Elastic and Inelastic Scattering of $40-\mathrm{MeV}$ Alpha Parti . cles by Carbon, Nitrogen, Oxygen, and Argon
Ispra report

EUR-2188.E;CONF64705-1(1965)

R.Colella, A.Merlini

J Phys Chem 68,310

(1964) J.Fock

et al

Phys Rev 133,B911 (1964) P.H.Stelson, F.K.McGowan

"Direct Interactions and Nuclear Reaction Mechanisms" (1963) neutrons, spectra p.282, Gordon and Breach Publishers D.M.Drake et al

Lawrence Berkeley Lab report UCRL-10783(1963) Thesis, B.D.Wilkins

Zhur Eksptl 1

Teoret Fiz 40,1263 (1961) V.V.Kuznetsov

Aeronautical Research Lab report ARL-TN-60-134 (1960) D.R.Locker

Phys Rev 117,801 (1960) J.L.Yntema et ai

ionization, transport theory

radiation effects, $\mathrm{x}$-radiation

optical model

ionization, slowdown

Nucl Phys 12,1 (1959) A.I.Yavin, G.W.Farwell

direct reactions 
Table IV (Cont.)

$9 \mathrm{Be}$ Total Neutron Yields from

10,11B Light Elements Under Proton

$7_{\mathrm{Li}}$

$238 \mathrm{U}$

$\mathrm{Al}, \mathrm{Th}$

$\mathrm{V}$

$\mathrm{Au}, \mathrm{Pb}$

${ }^{9} \mathrm{Be}, 10,11 \mathrm{~B}$

$12 \mathrm{C}$

$\mathrm{Cu}$

Ag

$\mathrm{Pb}, \mathrm{Ta}, \mathrm{Th}$

$\mathrm{Al}, \mathrm{Ni}, \mathrm{U}$

Th,U and Alpha Bombardment

Several Spallation Reactions of Uranium-238 Plus Helium-4

Inelastic Scattering of $\mathbf{4 0 . 2 -}$

MeV Alpha Particles

Elastic Scattering of $48-\mathrm{MeV}$

Alpha Particles

Neutrons and Gamma Rays from the Alpha-Particle Bombardment of $9 \mathrm{Be}, 10,11 \mathrm{~B}, 13 \mathrm{C}$ and $18 \mathrm{O}$

Protons from the Bombardment of Several Elements with 40MeV Alpha Particles

Elastic Scattering of Intermediate-Energy Alpha Particles by Heavy Nuclei

Analysis of Secondary Particles Resulting from HighEnergy Nuclear Bombardment

Some Studies of the Products of the High Energy Fission Process
Phys Rev 114,571 (1959) J.H.Gibbons, R.L.Macklin

Berkeley Radiation

Lab report

UCRL-8186(1958)

Thesis, J.A.Coleman

Phys Rev 106,256

(1957) G.Igo

inelastic

scattering

Phys Rev 101,636

(1956) R.E.Ellis,

L.Schecter

Phys Rev 102,1348

(1956) T.W.Bonner

et al

Phys Rev 100,1309

(1955) R.M.Eisberg

et al

Phys Rev 95,1212

(1954) G.W.Farwell,

H.E.Wegner

Berkeley Radiation

Lab report

evaporation

UCRL-2258(1953)

Thesis, R.W.Deutsch

Livermore Research

Lab report AECD-

fission products spectra

Fermi Gis Model model
3525(1952) M.Lindner,

R.N.Osbome 
$28 \mathrm{Si} \quad$ Neutrons from the Disintegration of the Separated Isotopes of Silicon by Deuterons

U

$27 \mathrm{Al}$

$181 \mathrm{Ta}$

${ }^{9} \mathrm{Be}, 63 \mathrm{Cu}$

$\mathrm{Sn}, \mathrm{U}$

$\mathrm{Be}, \mathrm{Bi}$

C,U

$\mathrm{Bi}, \mathrm{Pb}$
High Energy Neutron Yields and Multiple Traversals of a Cyclotron Beam Through Thin Targets

Angular Distribution of Fast Neutrons from Deuteron Bombardment

Angular Distribution of Neutrons from Targets

Bombarded by 20-MeV Deuterons

Relative High Energy Neutron Yields from Targets Bombarded with Protons and Deuterons

High Energy Induced Fission of Bismuth and Lead
Phys Rev $\underline{85}, 193$

(1952)

C.E.Mandeville

et al

Berkeley Radiation

Lab report

UCRL-1398(1951)

Thesis, WJ.Knox

Phys Rev 82,266

(1951) K.H.Sun

et al

Berkeley Radiation

Lab report

UCRL-1204(1951)

L.Schecter

Berkeley Radiation

Lab report

UCRL-440(Rev)

(1950) WJ.Knox

Phys Rev 76,628

(1949) R.H.Goecker-

mann, I.PerIman recoils

fast neutrons, stripping

fast neutrons

6

fast neutrons

fast neutrons stripping

evaporation, spectra 
$\mathrm{Al}, \mathrm{Ni}$

Ag,U

U

Th, U
Secondary, Particles Resulting

from $375 \mathrm{MeV}$ Alpha Bombardment of Nuclei

Observations of Lithium and Beryllium Nuclei Ejected from Heavy Nuclei by High Energy Particles

High Energy Spallation and Fission Products of Uranium

Radioactivities Produced in the Platinum Group by Bombardment of Uranium with 400 Million Electron Volt Helium Ions
Berkeley Radiation

Lab report

UCRL-2098(1953)

R.W.Deutsch

Berkeley Radiation

Lab report

UCRL-667(Rev)(1950)

L.Marquez, I.Perlman

Phys Rev 74,1189

(1948) P.R.O'Connor, G.T.Seaborg

Berkeley Radiation

thicimal neutrons

neutrons

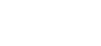

Lab report, UCRL. 123(1948) R.D.Wolfe, Thesis 


\section{$6,7_{\mathrm{Li}}$ INDUCED REACTIONS}

TARGET

TITLE

REFERENCE

Conf on Nucl Spect

\& Atomic Nucl

Struct., Alma-Ata

USSR,INIS-SU-282

pp 460-461 (1984)

N.Tompson

$208 \mathrm{~Pb}$

Inclusive Gamma-Ray

Spectra From the $6 \mathrm{Li}+$

$208 \mathrm{~Pb}$ Interaction at

12-26 MeV/A

Heavy-Ion Total Reaction

Cross Sections

Inclusive Gamma-Ray

Spectra from $208 \mathrm{~Pb}$

Bombarded by 73-156

MeV 6Li Ions

Verhandlunger Der

Deutschen Physik-

alischen Gesell-

schaft 19,907

(1984)(E.Ger.)

M.Albinska et al

Zeit fur Physik

A317,75(1984)

S.K.Gupta,S.Kailas

Karlsruhe report

KFK-3621,Annual

Rept on Nucl Phy-

sics Activities

7-1-82 to 6-30-83

(1983)p.45

M.Albinska et al

40 Ca Pre-Equilibrium Emission

$165 \mathrm{Ho}$

in Heavy Ion Reactions

$28 \mathrm{Si}$

Decay of the Nuclear Continuum of $28 \mathrm{Si}$ Excited Via Inelastic $6_{\mathrm{Li}}$ Scattering

${ }^{40} \mathrm{Ca}$

Inclusive Break-Up Reactions of $6 \mathrm{Li}$ at an Incident Energy of $26 \mathrm{MeV} /$ Nucleon
Phys Rev C28,2173 (1983) H.Machner et al

Max Planck Inst. Annual report INIS-MF-8299

pp.94-95(1983)

R.Clausing et al

Karlsruhe report

KFK-3277(1982)

R.Shyam et al
DESCRIPTORS

elastic

scattering, optical model

inclusive interactions, gamma cascades, gamma spectra

total cross sections

inclusive interactions, gamma spectra

precompoundnucleus emission

quasi-elastic scattering

inclusive interactions, precompoundnucieus 


\begin{tabular}{|c|c|c|c|}
\hline $\begin{array}{l}{ }^{40} \mathrm{Ca},{ }^{12} \mathrm{C} \\
208_{\mathrm{Pb}} \\
90 \mathrm{Zr}\end{array}$ & $\begin{array}{l}\text { Optical Model Studies } \\
\text { of } 6 \mathrm{Li} \text { Elastic Scattering } \\
\text { at } 156 \mathrm{MeV}\end{array}$ & $\begin{array}{l}\text { Nucl Phys A388, } 173 \\
\text { (1982) J.Cook et al }\end{array}$ & $\begin{array}{l}\text { elastic } \\
\text { scattering }\end{array}$ \\
\hline $\begin{array}{l}\text { heavy, } \\
\text { inter- } \\
\text { mediate } \\
\text { mass nuclei }\end{array}$ & $\begin{array}{l}\text { Global Optical-Model } \\
\text { Potentials for the } \\
\text { Elastic Scattering of } \\
6,7 \mathrm{Li} \text { Projectiles }\end{array}$ & $\begin{array}{l}\text { Nucl Phys } \underline{A} 388,153 \\
\text { (1982) J. Cook et al }\end{array}$ & $\begin{array}{l}\text { inclusive } \\
\text { interactions }\end{array}$ \\
\hline${ }^{40} \mathrm{Ca}$ & $\begin{array}{l}\text { Inclusive Break-Up } \\
\text { Reactions of } 6 \mathrm{Li} \text { at an } \\
\text { Incident Energy of } \\
26 \mathrm{MeV} / \text { Nucleon }\end{array}$ & $\begin{array}{l}\text { Nucl Phys A382,296 } \\
\text { (1982) B.Neumann } \\
\text { et al }\end{array}$ & $\begin{array}{l}\text { breakup } \\
\text { reactions }\end{array}$ \\
\hline \multirow[t]{2}{*}{$\begin{array}{l}209 \mathrm{Bi} \\
197 \mathrm{Au} \\
180 \mathrm{Hf} \\
198 \mathrm{Pt}\end{array}$} & $\begin{array}{l}\text { Multiplicity of K X Rays } \\
\text { and Collective Structure } \\
\text { in the Transitional Nuclei } \\
\text { with A Approx. = } 200\end{array}$ & $\begin{array}{l}\text { Phys Rev C25, } 1355 \\
\text { (1982) H.J.Karwowski } \\
\text { et al }\end{array}$ & $\begin{array}{l}\mathrm{K} \text { conversion, } \\
\mathrm{X} \text { radiation }\end{array}$ \\
\hline & $\begin{array}{l}\text { Continuous Charged-Particle } \\
\text { Spectra from } 40 \mathrm{Ca}+6 \mathrm{Li} \\
\text { at an Incident Energy of } \\
26 \mathrm{MeV} / \text { Nucleon }\end{array}$ & $\begin{array}{l}\text { Annual Report } \\
\text { JUEL-SPEZ-146, } \\
\text { p.31(1982) } \\
\text { H.Rebel et al }\end{array}$ & $\begin{array}{l}\text { inclusive } \\
\text { interactions }\end{array}$ \\
\hline $\begin{array}{l}40 \mathrm{Ca} \\
208 \mathrm{~Pb} \\
58 \mathrm{Ni}, 28 \mathrm{Si}\end{array}$ & $\begin{array}{l}\text { Energy Dependence of } 6 \mathrm{Li} \\
\text { Optical Potentials }\end{array}$ & $\begin{array}{l}\text { Nucl Phys } \underline{A} 375,238 \\
\text { (1982) J.Cook }\end{array}$ & $\begin{array}{l}\text { elastic } \\
\text { scattering }\end{array}$ \\
\hline $40 \mathrm{Ca}$ & $\begin{array}{l}\text { Continuous Particle Spectra } \\
\text { from }(6 \mathrm{Li}+40 \mathrm{Ca}) \text { Reactions } \\
\text { at } \mathrm{E}_{\mathrm{Li}}=156 \mathrm{MeV} \text { and the } \\
\text { Complex Structure of the } \\
\text { Triton Component }\end{array}$ & $\begin{array}{l}\text { Karlsruhe Annual } \\
\text { report KFK-3068 } \\
\text { pp.49-50(1980) } \\
\text { B.Neumann et al }\end{array}$ & $\begin{array}{l}\text { breakup } \\
\text { reactions, } \\
\text { inclusive } \\
\text { interactions }\end{array}$ \\
\hline $\begin{array}{l}12 \mathrm{C} \\
208 \mathrm{~Pb} \\
60 \mathrm{Ni}\end{array}$ & $\begin{array}{l}\text { Projectile Break-Up in } \\
\text { Continuous Particle Spectra } \\
\text { from Nuclear Reactions } \\
\text { Induced by } 156 \mathrm{MeV} 6_{\mathrm{Li}}\end{array}$ & $\begin{array}{l}\text { Karlsruhe report } \\
\text { KFK-3068 pp. } 48-49 \\
\text { (1981) H.Klewe- } \\
\text { Nebenius et al }\end{array}$ & $\begin{array}{l}\text { breakup } \\
\text { reactions }\end{array}$ \\
\hline${ }^{197} \mathrm{Au}$ & $\begin{array}{l}\text { Nucleon Spectra from } \\
\text { Heavy Ion Coilision Prior } \\
\text { to Equilibrium }\end{array}$ & $\begin{array}{l}\text { Phys Lett B98, } 240 \\
\text { (1981) P.Mooney } \\
\text { et al }\end{array}$ & $\begin{array}{l}\text { precompound- } \\
\text { nucleus }\end{array}$ \\
\hline $\begin{array}{l}12 \mathrm{C} \\
208 \mathrm{~Pb} \\
120 \mathrm{Sn}\end{array}$ & $\begin{array}{l}\text { Projectile Break-Up in } \\
\text { Continuous Particle from } \\
\text { Spectra Nuclear Reactions } \\
\text { Induced by } 156 \mathrm{MeV} 6 \mathrm{Li}\end{array}$ & $\begin{array}{l}\text { Zeit fur Physik } \\
\text { A296,113(1980) } \\
\text { B.Neumann et al }\end{array}$ & energy spectra \\
\hline $28 \mathrm{Si}$ & $\begin{array}{l}\text { Optical Potential for } 6 \mathrm{Li} \\
+28 \mathrm{Si} \text { Elastic Scattering } \\
\text { at } 154 \mathrm{MeV}\end{array}$ & $\begin{array}{l}\text { Phys Rev C21,1656 } \\
\text { (1980) P.Schwandt } \\
\text { et al }\end{array}$ & $\begin{array}{l}\text { elastic } \\
\text { scattering }\end{array}$ \\
\hline
\end{tabular}




$\begin{array}{ll}{ }_{12 \mathrm{C}, 197} \mathrm{Au} & \text { Nuclear Reactions Using } \\ 208 \mathrm{~Pb} & 156 \mathrm{MeV}-6 \mathrm{Li} \text { Ions }\end{array}$

193Ir

${ }^{59} \mathrm{Co}$

$59 \mathrm{Co}$

$27 \mathrm{Al}$

${ }^{59} \mathrm{Co}$

$\begin{array}{ll}27 \mathrm{Al} & \text { Elastic and Inelastic Scatter- } \\ 48 \mathrm{Ca} & \text { ing of } 88 \mathrm{MeV} 6 \mathrm{Li} \text { Ions } \\ 197 \mathrm{Au} & \\ & \\ 27 \mathrm{Al} & \begin{array}{l}\text { Optical-Model Analysis of } 7 \mathrm{Li} \\ +25 \mathrm{Mg} \text { and } 7 \mathrm{Li}+27 \mathrm{Al} \text { Elastic } \\ \end{array} \\ & \text { Scattering at } 89 \mathrm{MeV}\end{array}$

Verhandlungen Der

Deutschen Physi-

kalischen Gesell-

schaft (E.Ger.) 6,

947(1977)

J.Buschmann et al

Zeit fur Physik

A280,61(1977)

J.Kropp et al

Bull Acad Sci USSR

Phys Series

47,2177(1983)

O.V.Bochkarev et al

INIS-SU-282 p.383

34. Conf on Nucl

Spect \& Nucl Struct 1984,Alma-Ata, USSR, Yu.A.Glukhov et al

Nucl Phys A391,458

(1982) S.L.Tabor ot al

Nucl Instr Meth

200,285(1982)

J.H.Dave, C.R.Gotld

INIS-MF-6160 p.626

30th Conf Nucl

Spect \& Nucl Struct

Leningrad,USSR

Mar 18, 1980

O.V.Bovhkarev et al

Nucl Phys A356,235

(1981) C.B.Fulmer et al

Nuc! Phys A357,246

(1951) J.Cook et al elastic

mestrons, proton spectra

precompoundnucleus, $x$-radiation

energy spectra

precompoundmucleus emission

breakup reactions

fast neutrows

precompound-

muclews emission

optical models

scattering 


\begin{tabular}{|c|c|c|c|}
\hline $118 \mathrm{Sn}$ & Continuum Break-Up of $6 \mathrm{Li}$ & $\begin{array}{l}\text { Phys Lett } \mathrm{B} 96,47 \\
\text { 1980) H.Gemmeke et al }\end{array}$ & breakup reactions \\
\hline $40 \mathrm{Ca}$ & $\begin{array}{l}\text { Phenomenological and Micro- } \\
\text { scopic Optical Potentials for } \\
88 \mathrm{MeV} 7 \mathrm{Li} \text { Scattering }\end{array}$ & $\begin{array}{l}\text { J of Physics } \mathrm{G6}, 501 \\
\text { (1980) M.r.Steeden } \\
\text { et al }\end{array}$ & $\begin{array}{l}\text { elastic } \\
\text { scattering }\end{array}$ \\
\hline $\begin{array}{l}197 \mathrm{Au} \\
208 \mathrm{Pt} \\
181 \mathrm{Ta}\end{array}$ & $\begin{array}{l}\text { Multiplicity of } \mathrm{K} \times \text { Rays } \\
\text { Emitted in }(6 \mathrm{Li}, \mathrm{xn}) \text { Reactions }\end{array}$ & $\begin{array}{l}\text { Argonne Nat. Lab } \\
\text { report ANL/PHY-79. } \\
\text { 4-519(1979) } \\
\text { H.J.Karwowski et al }\end{array}$ & $\begin{array}{l}\text { gamma spectra, } \\
\text { x-ray spectra }\end{array}$ \\
\hline $\begin{array}{l}197 \mathrm{Au} \\
56 \mathrm{Fe}\end{array}$ & $\begin{array}{l}\text { Mechanisms of Production of } \\
\text { Fust Particles with } Z=1,2 \text { in } \\
6 \text { Li-Induced Reactions Far } \\
\text { Above the Coulomb Barrier }\end{array}$ & $\begin{array}{l}\text { Phys Rev C21,179 } \\
\text { (1980) C.M.Castaneda } \\
\text { et al }\end{array}$ & gamma spectra \\
\hline $13 \mathrm{C}$ & $\begin{array}{l}\text { De-Excitation Processes in the } \\
\text { Compound Nucieus Investigated } \\
\text { by a Charged Particle-Gamma } \\
\text { Correlation Method }\end{array}$ & $\begin{array}{l}\text { Dubna report JINR- } \\
\text { D-9682,p.143(1976) } \\
\text { M.A.Deleplanque et al }\end{array}$ & stripping \\
\hline $\begin{array}{l}197 \mathrm{Au} \\
208 \mathrm{~Pb}\end{array}$ & $\begin{array}{l}\text { Multiplicity of KX Rays } \\
\text { Emitted in }(6 \mathrm{Li}, \mathrm{xn}) \text { Reactions }\end{array}$ & $\begin{array}{l}\text { Phys Rev Lett } 42 \text {, } \\
\text { 1732(1979) } \\
\text { H.J.Karwowski et al }\end{array}$ & K-conversion \\
\hline $197 \mathrm{Au}$ & $\begin{array}{l}\text { Gamma-Rays from the Transfer } \\
\text { of Alpha and D Fragments in } \\
\text { in } 6 \mathrm{Li} \text { Induced Reactions Far } \\
\text { Above the Coulomb Barrier }\end{array}$ & $\begin{array}{l}\text { Phys Lett } \underline{B 77}, 371 \\
\text { (1978) C.M.Cas- } \\
\text { taneda et al }\end{array}$ & $\begin{array}{l}\text { breakup reactions, } \\
\text { gamma spectra }\end{array}$ \\
\hline $54,56 \mathrm{Fe}$ & $\begin{array}{l}\text { Fusion and Nonfusion Processes } \\
\text { in Reactions Induced by } 10-100 \\
\text { MeV 6Li Ions with Fe Targets }\end{array}$ & $\begin{array}{l}\text { Phys Rev C19,724 } \\
\text { (1979) } \\
\text { J.Jastrzebski et al }\end{array}$ & $\begin{array}{l}\text { precompound- } \\
\text { nucleus emission }\end{array}$ \\
\hline $\begin{array}{l}142,146 \mathrm{Nd} \\
150 \mathrm{Nd}\end{array}$ & $\begin{array}{l}\text { Investigation of 54- to 99- } \\
\text { MeV 6 Li-Induced Reactions } \\
\text { on Rare Earth Targets }\end{array}$ & $\begin{array}{l}\text { Phys Rev C17, } 1001 \\
\text { (1978) J.W.Mihelich } \\
\text { et al }\end{array}$ & neutron spectra \\
\hline
\end{tabular}


104, 106Pd

$12 \mathrm{C}, 160$

$40 \mathrm{Ca}, 58 \mathrm{Ni}$

$74 \mathrm{Ge}, 166 \mathrm{Er}$

${ }^{10} \mathrm{~B}, 12 \mathrm{C}$

$9 \mathrm{Be}, 28 \mathrm{Si}$

160

$10 \mathrm{~B}, 12 \mathrm{C}$

$28 \mathrm{Si}$

$238 \mathrm{U}$

$232 \mathrm{Th}$

$197 \mathrm{Au}$

$7 \mathrm{Li}$

${ }^{197} \mathrm{Au}$

${ }^{209} \mathrm{Bi}$
Investigation of $6 \mathrm{Li}$-Induced

Reactions from 54 to $99 \mathrm{MeV}$

on Pd Targets

$6 \mathrm{Li}$ Elastic Scattering on $12 \mathrm{C}$, $160,58 \mathrm{Ni}, 74 \mathrm{Ge}, 124 \mathrm{Sn}, 166 \mathrm{Er}$ and $208 \mathrm{~Pb}$ at $\mathrm{E}(6 \mathrm{Li})=50.6 \mathrm{MeV}$

Elastic Scattering of Lithium by $9 \mathrm{Be}, 10 \mathrm{~B}, 12 \mathrm{C}, 16 \mathrm{O}$, and

$28 \mathrm{Si}$ from 4 to $63 \mathrm{MeV}$

Elastic Scattering of Lithium by $9 \mathrm{Be}, 10 \mathrm{~B}, 12 \mathrm{C}, 13 \mathrm{C}, 16 \mathrm{O}$, and $28 \mathrm{Si}$ from 4 to $63 \mathrm{MeV}$

$6 \mathrm{Li}$ and $7 \mathrm{Li}$ Induced Fission of $232 \mathrm{Th}$ and $238 \mathrm{U}$

Study of Projectile-Target Interaction Processes Around the Coulomb Barrier. Breakup in Flight of the Projectile;

Elastic and Inelastic

Scattering

$7 \mathrm{Li}+7 \mathrm{Li}$ Reaction Studies Leading to Multi-Neutron Final States

Reactions Produced by $7 \mathrm{Li}$ on 197 Au

$6 \mathrm{Li}, 7 \mathrm{Li}$ induced Reactions on $209 \mathrm{Bi}$

6,7 $\mathrm{Li}$ Induced Fission of $232 \mathrm{Th}$ and $238 \mathrm{U}$
Phys Rev C16, 1437

(1977)

C.M.Castaneda et al

Nucl Phys A273,243

(1976) L.T.Chua

et al

Thesis(1975) U of

Iowa J.E.Poling

Phys Rev $\underline{\text { C13 }}, 648$

(1976) J.E.Poling

et al

Phys Rev C12,42

(1975)

H.Freiesieben et al

Bordeaux Univ

Thesis, CEN-TS-74-1

(1974) G.Sousbie

Phys Lett B53,247

(1974) J.Cerny et al

Proc Int Conf on

Reactions Between

Complex Nuclei Vol

I and Suppl Jun 10, 1974 Nashville,Tn,

J.L.Quebert et al

Phys Rev C10,245

(1974)

H.Freiesleben et al

Univ of Rochester

report COO-3496-44

pp.85-91(1974)

H.Freiesleben,

J.R.Huizenga precompoundnucleus emission, gamma spectra

elastic scattering

elastic scattering

elastic scattering

fission, transfer reactions

breakup reactions

energy spectra

one-nucleon transfer reactions

excitation functions

excitation

functions 
238U . Spaliation Reactions of $6 \mathrm{Li}$ with 238U

${ }^{12} \mathrm{C}, 26 \mathrm{Mg}$

$28 \mathrm{Si}$

232Th

$238 \mathrm{U}$

$238 \mathrm{U}$

$238 \mathrm{U}$

$12 \mathrm{C}, 160$

$40 \mathrm{Ca}, 12 \mathrm{C}$

$160,28 \mathrm{Si}$

$197 \mathrm{Au}$
Lithium Elastic and Inelastic Scattering and Lithium-Induced Single Nucleon Transfer Reactions

Total Reaction Cross Section of $6 \mathrm{~L}_{1}$ and $7 \mathrm{Li}$ on $232 \mathrm{Th}$ and $238 \mathrm{U}$

Angular Distributions for $6 \mathrm{Li}$ and $7 \mathrm{Li}$ Induced Fission of 232 Th and 238U

Elastic Scattering of Lithium on 232 Th and 238U

Elastic Scattering of $6 \mathrm{Li}$ on $12 \mathrm{C}$ and 160

Elastic Scattering of $6 \mathrm{Li}$ Ions

In Beam Studies of natIr, $197 \mathrm{Au}\left(6 \mathrm{Li}, \mathrm{xn}^{+} \mathrm{yp}\right)$ Reactions Induced by $6 \mathrm{Li}$-Ions of up to $156 \mathrm{MeV}$
Purdue Univ report

COO-1716-17 p.15

(1974) R.C.Rudy

Nucl Phys A212,573

(1973) P.Schumacher et al

elastic scattering, stripping

Univ of Kochester report COO-3496-29 p. 20(1973) H.Freissleben et al

Univ of Rochester report COO-3496-29

p.14(1973)

H.Freiesleben et al

Univ of Rochester report COO-3496-29

p.2(1973)

HFreiesleben et al

J Phys (Paris)

Colloq 6, 161(1971)

V.I.Chuev et al

Izv Akad Nauk SSSR

Ser Fiz 35,2399

(1971) V.V.Davydov et al

Julich report

JUL-CONF-16(V.1) ,

p.88(1975)

J.Buschmarin et al elastic

scattering

excitation

functions

energy

dependence

optical models

optical model

excitation

functions 
TABLE VI

EXPERIMENTAL DATA REFERENCE TABLE FOR PROTON

ELASTIC SCATTERING AT MEDIUM ENERGIES

Target

Energy

Angular Range

Reference+

$(\mathrm{MeV})$

$\sigma(\theta)$

$A(\theta)$

$12 \mathrm{C}$

65
100
122
152
156
160
185
200
613
800
800
1000
1040

$15-115^{\circ}$

$15-115^{\circ}$

NIM 169, 589 (1980)

$5-90^{\circ}$

$7.143^{\circ}$

$7-143^{\circ}$

CJP 44, 2961 (1966)

PR C27, 459 (1983)

NP 80, 625 (1966)

$4-65^{\circ}$

$8-65^{\circ}$

NP A221, 403 (1974)

$8.143^{\circ}$

PR C27, 459 (1983)

NP A 319, 377 (1979)

$7-85^{\circ}$

$2.65^{\circ}$

$7-115^{\circ}$

PR C27, 459 (1983)

$7-157^{\circ}$

-....-

Ref. (a)

PR C23, 2599 (1981)

$4-67^{\circ}$

$\begin{array}{ll}2-41^{\circ} & 2-30^{\circ} \\ & 3-19^{\circ}\end{array}$

PR C18, 1756 (1978)

PL 90B, 364 (1980)

4-30

PL 45B, 119 (1973)

$13 \mathrm{C}$

135

200

800

9-82

$7-115^{\circ}$

$10-40^{\circ}$

$7-115^{\circ}$

IUCF Ann.Rep. 1980,21

PR C23, 616 (1981)

PR C18, 1436 (1978)

$14 N$

144

160

800

$6-62^{\circ}$

$8-48^{\circ}$

$8-48^{\circ}$

PR C21, 25 (1980)

IUCF Ann.Rep. 1980,24

$6-.32^{\circ}$

-...-.

PR C25, 2550 (1982)

160

65

100

135

200

613

800

1000

1040

$24 \mathrm{Mg} \quad .65$

100

135

155

800

$26 \mathrm{Mg}$

65

800

$27 \mathrm{Al}$

65

156

185

\begin{tabular}{|c|c|}
\hline $18-82^{\circ}$ & $18-72^{\circ}$ \\
\hline $6-84^{\circ}$ & ------ \\
\hline $8-91^{\circ}$ & $8-91^{\circ}$ \\
\hline $6-143^{\circ}$ & $6.143^{\circ}$ \\
\hline $4-34^{\circ}$ & -..... \\
\hline $6-26^{\circ}$ & $6-26^{\circ}$ \\
\hline -.--- & $5-20^{\circ}$ \\
\hline $3-39^{\circ}$ & -...-. \\
\hline
\end{tabular}

$14-87^{\circ}$

$10-60^{\circ}$

$8-57^{\circ}$

-..-.

$5-31^{\circ}$

$20-80^{\circ}$

$5-31^{\circ}$

$20-80^{\circ}$

$8-72^{\circ}$ $14-72^{\circ}$

$8-57^{\circ}$

$4-58^{\circ}$

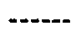

$15-80^{\circ}$

$15-30^{\circ}$

$4-40^{\circ}$
PR C26, 944 (1982)

CJP 48, 765 (1970)

PRL 45, 2012 (1980)

IUCF Ann.Rep. 1981,1

Ref. (a)

PRL 43, 421 (1979)

PL 90B, 364 (1980)

Ref. (a.)

PR C26, 944 (1982)

NP A193, 438 (1972)

PR C26, 55 (1982)

NP A112, 417 (1968)

PR C25, 422 (1982)

RCNP Ann.Rep. 1979,20

PR C25, 422 (1982)

RCNP Ann.Rep. 1979,20

NP A221, 403 (1974)

PS 4, 235 (1971) 
Table VI (Cont.)

\begin{tabular}{|c|c|c|c|c|}
\hline & $(\mathrm{MeV})$ & $\sigma(\theta)$ & $A(\theta)$ & \\
\hline \multirow[t]{5}{*}{$28 \mathrm{Si}$} & 65 & $14-90^{\circ}$ & $14-74^{\circ}$ & PR C26, 944 (1982) \\
\hline & 100 & $10-60^{\circ}$ & $\cdots$ & NP A193, 438 (1972) \\
\hline & 135 & $7-89^{\circ}$ & $8-62^{\circ}$ & PR C26, 55 (1982) \\
\hline & 155 & -.--- & $4-58^{\circ}$ & NP A112, 417 (1968) \\
\hline & 180 & $9-92^{\circ}$ & $9-92^{\circ}$ & IUCF Ann.Rep. 1981,1 \\
\hline \multirow[t]{2}{*}{$30 \mathrm{Si}$} & 650 & $5-20^{\circ}$ & $5-20^{\circ}$ & BAPS 27,730 (1982) \\
\hline & 800 & $5-20^{\circ}$ & $5-20^{\circ}$ & BAPS $27,730(1982)$ \\
\hline $32 S$ & 155 & $-\cdots$ & $4-58^{\circ}$ & A112, 417 (1968) \\
\hline \multirow[t]{2}{*}{$34 S$} & 650 & $5-20^{\circ}$ & $5-20^{\circ}$ & BAPS 27,730 (1982) \\
\hline & 800 & $5-20^{\circ}$ & $5-20^{\circ}$ & BAPS 27,730 (1982) \\
\hline \multirow[t]{17}{*}{$40 \mathrm{Ca}$} & 65 & $16-80^{\circ}$ & $16-66^{\circ}$ & PR C26, 944 (1982) \\
\hline & 80 & $5-95^{\circ}$ & $8-59^{\circ}$ & PR C26, 55 (1982) \\
\hline & 135 & $9-95^{\circ}$ & $-\cdots$ & PR C23, 1023 (1981) \\
\hline & 155 & $-\cdots$ & $4-61^{\circ}$ & NP A112, 417 (1968) \\
\hline & 156 & $5-53^{\circ}$ & -...... & NP A221, 403 (1974) \\
\hline & 160 & $8-88^{\circ}$ & $\cdots-\cdots$ & PR C23, 1023 (1981) \\
\hline & 182 & $8-69^{\circ}$ & $8-69^{\circ}$ & PR C26, 55 (1982) \\
\hline & 185 & $5-62^{\circ}$ & $2-41^{\circ}$ & PS 4, $235(1971)$ \\
\hline & 200 & $3-55^{\circ}$ & $3-55^{\circ}$ & Ref. (b) \\
\hline & 300 & $3-50^{\circ}$ & $3-50^{\circ}$ & Ref. (b) \\
\hline & 400 & $3-52^{\circ}$ & $3-52^{\circ}$ & Ref. (b) \\
\hline & 500 & $3-43^{\circ}$ & $3-43^{\circ}$ & Ref. (b) \\
\hline & 500 & $6-30^{\circ}$ & $6-30^{\circ}$ & PRL 47, 1437 (1981) \\
\hline & 613 & $3-30^{\circ}$ & -...-- & Ref. (a) \\
\hline & 800 & $2-42^{\circ}$ & $2.31^{\circ}$ & PR C.25, 2563 (1982) \\
\hline & 1000 & ----- & $2-14^{\circ}$ & PL 90B, 364 (1980) \\
\hline & 1044 & $4-20^{\circ}$ & $-\cdots--$ & NP A274, 443 (1976) \\
\hline \multirow[t]{4}{*}{${ }^{42} \mathrm{Ca}$} & 650 & $5-20^{\circ}$ & $5-20^{\circ}$ & BAPS 27,730 (1982) \\
\hline & 800 & $5-20^{\circ}$ & $5-20^{\circ}$ & BAPS 27,730 (1982) \\
\hline & 800 & $3-23^{\circ}$ & $4-20^{\circ}$ & PL 81B, 151 (1979) \\
\hline & 1044 & $4-18^{\circ}$ & ----- & NP A274, 443 (1976) \\
\hline \multirow[t]{3}{*}{${ }^{44} \mathrm{Ca}$} & 65 & $16-68^{\circ}$ & $16-70^{\circ}$ & PR C26, 944 (1982) \\
\hline & 800 & $3-24^{\circ}$ & $3-24^{\circ}$ & PL 81B, 151 (1979) \\
\hline & 1044 & $4-18^{\circ}$ & -..--- & NP A274, 443 (1976) \\
\hline \multirow[t]{4}{*}{${ }^{48} \mathrm{Ca}$} & 65 & $24-68^{\circ}$ & $24-70^{\circ}$ & PR C26, 944 (1982) \\
\hline & 500 & $6-28^{\circ}$ & $6-28^{\circ}$ & PRL 47, 1437 (1981) \\
\hline & 800 & $3-23^{\circ}$ & $3-21^{\circ}$ & PL 81B, 151 (1979) \\
\hline & 1044 & $4-20^{\circ}$ & $\ldots$ & NP A274, 443 (1976) \\
\hline \multirow[t]{3}{*}{${ }^{48} \mathrm{Ti}$} & 65 & $16-68^{\circ}$ & $16-70^{\circ}$ & PR C26, 944 (1982) \\
\hline & 156 & $5-42^{\circ}$ & - & NP A221, 403 (1974) \\
\hline & 1044 & $4-20^{\circ}$ & - & NP A274, 443 (1976) \\
\hline
\end{tabular}


Table VI (Cont.)

\begin{tabular}{|c|c|c|c|c|}
\hline & $(\mathrm{MeV})$ & $\sigma(\theta)$ & $A(\theta)$ & \\
\hline $54 \mathrm{Fe}$ & $\begin{array}{r}65 \\
800\end{array}$ & $\begin{array}{r}16-68^{\circ} \\
3-22^{\circ}\end{array}$ & $\begin{array}{r}16-70^{\circ} \\
3-20^{\circ}\end{array}$ & $\begin{array}{l}\text { PR C26, } 944(1982) \\
\text { PL 79B. } 376(1978)\end{array}$ \\
\hline $56 \mathrm{Fe}$ & $\begin{array}{r}65 \\
156 \\
185 \\
800\end{array}$ & $\begin{array}{r}14-68^{\circ} \\
8-42^{\circ} \\
10-66^{\circ} \\
3-32^{\circ}\end{array}$ & $\begin{array}{l}14-70^{\circ} \\
--32^{\circ} \\
3-24^{\circ}\end{array}$ & $\begin{array}{l}\text { PR C26, } 944(1982) \\
\text { NP A221, } 403(1974) \\
\text { PS 4, 235 (1971) } \\
\text { PL 79B, } 376(1978)\end{array}$ \\
\hline $58 \mathrm{Ni}$ & $\begin{array}{r}65 \\
100 \\
160 \\
178 \\
800 \\
1044\end{array}$ & $\begin{array}{c}14-68^{\circ} \\
8-129^{\circ} \\
5-45^{\circ} \\
6-60^{\circ} \\
2-32^{\circ} \\
4-24^{\circ}\end{array}$ & $\begin{array}{c}14-70^{\circ} \\
\cdots-38^{\circ} \\
2-21^{\circ} \\
\cdots\end{array}$ & $\begin{array}{l}\text { PR C26, } 944(1982) \\
\text { NP A301, } 349(1978) \\
\text { PR 140, B1237 (1965) } \\
\text { NP A322, } 285(1979) \\
\text { PL 79B, } 376(1978) \\
\text { PL 67B, } 402(1977)\end{array}$ \\
\hline $60 \mathrm{Ni}$ & $\begin{array}{r}65 \\
1044\end{array}$ & $\begin{array}{r}14-68^{\circ} \\
3-18^{\circ}\end{array}$ & $14-70^{\circ}$ & $\begin{array}{l}\text { PR C26, } 944 \text { (1982) } \\
\text { PL 67B. } 402(1977)\end{array}$ \\
\hline $62 \mathrm{Ni}$ & $\begin{array}{r}65 \\
156\end{array}$ & $\begin{array}{r}13-66^{\circ} \\
6-41^{\circ}\end{array}$ & $13-68^{\circ}$ & $\begin{array}{l}\text { PR C26, } 944(1982) \\
\text { NP A221, } 403 \text { (1974) }\end{array}$ \\
\hline $64 \mathrm{Ni}$ & $\begin{array}{r}65 \\
800 \\
1044\end{array}$ & $\begin{array}{r}13-68^{\circ} \\
3-25^{\circ} \\
4-16^{\circ}\end{array}$ & $\begin{array}{r}13-70^{\circ} \\
3-25^{\circ} \\
-\end{array}$ & $\begin{array}{l}\text { PR C26, } 944(1982) \\
\text { PL 79B, } 376(1978) \\
\text { PL 67B, } 402(1977)\end{array}$ \\
\hline $89 Y$ & $\begin{array}{r}65 \\
156 \\
185\end{array}$ & $\begin{array}{r}13-58^{\circ} \\
8-55^{\circ} \\
10-40^{\circ}\end{array}$ & $\begin{array}{l}13-60^{\circ} \\
5-36^{\circ}\end{array}$ & $\begin{array}{l}\text { PR C26, } 944(1982) \\
\text { NP A221, } 403 \text { (1974) } \\
\text { NP A305, } 333(1978)\end{array}$ \\
\hline $90 \mathrm{Zr}$ & $\begin{array}{r}65 \\
80 \\
99 \\
100 \\
135 \\
156 \\
160 \\
180 \\
185 \\
500 \\
800 \\
1000\end{array}$ & $\begin{array}{c}13-58^{\circ} \\
6-91^{\circ} \\
-15-77^{\circ} \\
6-126^{\circ} \\
8-41^{\circ} \\
5-82^{\circ} \\
6-86^{\circ} \\
10-40^{\circ} \\
6-30^{\circ} \\
2-30^{\circ} \\
4-17^{\circ}\end{array}$ & \begin{tabular}{c}
$13-60^{\circ}$ \\
$8-58^{\circ}$ \\
$8-59^{\circ}$ \\
\hdashline $11-59^{\circ}$ \\
\hdashline $12-44^{\circ}$ \\
\hdashline$--33^{\circ}$ \\
$6-30^{\circ}$ \\
$2-21^{\circ}$ \\
$3-11^{\circ}$
\end{tabular} & $\begin{array}{l}\text { PR C26, } 944(1982) \\
\text { PR C26, } 55(1982) \\
\text { PR C26, } 55(1982) \\
\text { NP A301, } 349(1978) \\
\text { PR C26, } 55(1982) \\
\text { NP A221, } 403(1974) \\
\text { PR C26, } 55(1982) \\
\text { PR C23, } 1023(1981) \\
\text { PS 4, 235 (1971) } \\
\text { PRL 47, } 1437(1981) \\
\text { PR C18, } 1756(1978) \\
\text { PL. 90B, } 364(1980)\end{array}$ \\
\hline $92 \mathrm{Zr}$ & 104 & $12-51^{\circ}$ & $12-51^{\circ}$ & PR C26, 55 (1982) \\
\hline $116 \mathrm{Sn}$ & 800 & $2-22^{\circ}$ & $2-22^{\circ}$ & PL 76B, 383 (1978) \\
\hline $120 \mathrm{Sn}$ & $\begin{array}{l}100 \\
104\end{array}$ & $\begin{array}{r}23-55^{\circ} \\
8-51^{\circ}\end{array}$ & $8-51^{\circ}$ & $\begin{array}{l}\text { NP A301, } 349 \text { (1978) } \\
\text { PR C26, } 55(1982)\end{array}$ \\
\hline
\end{tabular}


Table VI (Cont.)

\begin{tabular}{|c|c|c|c|c|}
\hline $120 \mathrm{Sn}$ & $\begin{array}{l}156 \\
160\end{array}$ & $\begin{array}{l}5-41^{\circ} \\
5-45^{\circ}\end{array}$ & $\cdots$ & $\begin{array}{l}\text { NP A221, } 403 \text { (1974) } \\
\text { PR 140, B1237 (1965) }\end{array}$ \\
\hline $124 \mathrm{Sn}$ & 800 & $2-22^{\circ}$ & $2-22^{\circ}$ & PL 76B, 383 (1978) \\
\hline $\begin{array}{l}144 \mathrm{Sm} \\
176 \mathrm{Yb}\end{array}$ & $\begin{array}{l}800 \\
800\end{array}$ & $\begin{array}{l}5-20^{\circ} \\
5-20^{\circ}\end{array}$ & $\cdots$ & $\begin{array}{l}\text { PR C22, } 1168(1980) \\
\text { PR C22, } 1168(1980)\end{array}$ \\
\hline $208 \mathrm{~Pb}$ & $\begin{array}{r}65 \\
80 \\
98 \\
100 \\
121 \\
155 \\
156 \\
160 \\
160 \\
182 \\
185 \\
200 \\
300 \\
400 \\
500 \\
500 \\
613 \\
800 \\
1000\end{array}$ & $\begin{array}{r}14-72^{\circ} \\
6-93^{\circ} \\
10-76^{\circ} \\
19-79^{\circ} \\
5-83^{\circ} \\
-\ldots-{ }^{\circ} \\
6-40^{\circ} \\
6-77^{\circ} \\
5-45^{\circ} \\
6-80^{\circ} \\
4-38^{\circ} \\
3-59^{\circ} \\
3-49^{\circ} \\
3-51^{\circ} \\
3-42^{\circ} \\
5-32^{\circ} \\
4-22^{\circ} \\
3-42^{\circ} \\
2-15^{\circ}\end{array}$ & \begin{tabular}{r}
$14-70^{\circ}$ \\
$8-58^{\circ}$ \\
$10-76^{\circ}$ \\
$\cdots$ \\
\hdashline$-\cdots$ \\
$5-40^{\circ}$ \\
$-\cdots$ \\
$8-53^{\circ}$ \\
$4-38^{\circ}$ \\
$3-59^{\circ}$ \\
$3-49^{\circ}$ \\
$3-51^{\circ}$ \\
$3-42^{\circ}$ \\
$5-32^{\circ}$ \\
$-2--^{\circ}$ \\
$2-32^{\circ}$ \\
$2-11^{\circ}$
\end{tabular} & $\begin{array}{l}\text { PR C26, } 944(1982) \\
\text { PR C26, } 55(1982) \\
\text { PR C26, } 55(1982) \\
\text { NP A301, } 349(1978) \\
\text { PR C23, } 1023(1981) \\
\text { JP 30, 13 (1969) } \\
\text { NP A221, } 403(1974) \\
\text { PR C23, } 1023(1981) \\
\text { PR 140, B1237(1965) } \\
\text { PR C26, } 55(1982) \\
\text { PR C10, } 307(1974) \\
\text { Ref. (b) } \\
\text { Ref. (b) } \\
\text { Ref. (b) } \\
\text { Ref. (b) } \\
\text { PRL 47, } 1437 \text { (1981) } \\
\text { Ref. (a) } \\
\text { PR C24, } 541 \text { (1981) } \\
\text { PL 90B, } 364 \text { (1980) }\end{array}$ \\
\hline $209_{\mathrm{Bi}}$ & $\begin{array}{r}65 \\
153 \\
156\end{array}$ & \begin{tabular}{c}
$13-72^{\circ}$ \\
\hdashline$-55^{\circ}$
\end{tabular} & $\begin{array}{r}13-72^{\circ} \\
5-56^{\circ} \\
-\cdots\end{array}$ & $\begin{array}{l}\text { PR C26, } 944(1982) \\
\text { NF 80, 625 (1966) } \\
\text { NP A221, } 403(1974)\end{array}$ \\
\hline
\end{tabular}

+ Journal Abbreviaton:

BAPS - Bulletin of the American Physical Society

CJP - Canadian Journal of Physics

JP - Journal de Physique

NIM - Nuclear Instruments and Methods

NP - Nuclear Physics

PL - Physics Letters

PR - Physical Review

PRL - Physical Review Letters

PS - Physica Scripta

+Laboratory Report Abbreviation: IUCF - Indiana Univ. Cyclotron Facility

RCNP - Reseach Center f. Nuclear Physics, Osaka, Japan

References:

(a) CEN Saclay Report DPh-N/ME/78-1

(b) AIP Conf. Proc. No.69 (Polarization Phenomena in

Nuclear Physics 1980), p.454 
EXPERIMENTAL DATA REFERENCE TABLE FOR

PROTON TOTAL REACTION CROSS SECTIONS AT MEDIUM ENERG:ES

\begin{tabular}{|c|c|c|}
\hline Target & $\frac{\text { Energy }}{(\mathrm{MeV})}$ & Reference $t$ \\
\hline $\mathrm{Li}$ & 179.6 & AF $19,527(1961)$ \\
\hline $\mathrm{Be}$ & $\begin{array}{l}99.3 \\
179.6 \\
232 \pm 20 \\
346 \pm 20 \\
464 \pm 20 \\
553 \pm 20\end{array}$ & $\begin{array}{l}\text { CJP 44,1847(1966) } \\
\text { AF } 19,527(1961) \\
\text { NP A183,81 (1972) } \\
\text { NP A183,81 (1972) } \\
\text { NP A183,81 (1972) } \\
\text { NP A183,81 (1972) }\end{array}$ \\
\hline $\begin{array}{r}12 \mathrm{C} \\
\mathrm{C}\end{array}$ & $\begin{array}{l}60 \\
77 \pm 5 \\
95 \pm 5 \\
99.1 \\
113 \pm 5 \\
133 \pm 5 \\
134 \pm 4 \\
179.6 \\
231 \pm 20 \\
345 \pm 20 \\
464 \pm 20 \\
552 \pm 20\end{array}$ & $\begin{array}{l}\text { PRL 22,1128 (1969) } \\
\text { NP 29,474 (1962) } \\
\text { NP 29,474 (1962) } \\
\text { CP 44,1847 (1966) } \\
\text { NP 29,474(1962) } \\
\text { NP 29,474(1962) } \\
\text { PPS(Lond.) 67,125 (1954) } \\
\text { AF 19,527(1961) } \\
\text { NP A183,81 (1972) } \\
\text { NP A183,81 (1972) } \\
\text { NP A183,81(1972) } \\
\text { NP A183,81 (1972) }\end{array}$ \\
\hline $\mathrm{F}$ & 99.1 & CJP 44,1847 (1966) \\
\hline $\mathbf{M g}$ & 99.4 & CJP 44,1847 (1966) \\
\hline $\begin{aligned} 27 \mathrm{Al} \\
\mathrm{Al}\end{aligned}$ & $\begin{array}{l}60 \\
77 \pm 5 \\
95 \pm 5 \\
99.7 \\
113 \pm 5 \\
133 \pm 5 \\
134 \pm 4 \\
179.6 \\
234 \pm 20 \\
348 \pm 20 \\
466 \pm 20 \\
554 \pm 20\end{array}$ & $\begin{array}{l}\text { PRL 22,1128 (1969) } \\
\text { NP 29,474 (1962) } \\
\text { NP 29,474 (1962) } \\
\text { CPP 44,1847 (1966) } \\
\text { NP 29,474 (1962) } \\
\text { NP 29,474 (1962) } \\
\text { PPS(Lond.) 67,125(1954) } \\
\text { AF 19,527(1961) } \\
\text { NP A183,81 (1972) } \\
\text { NP A 1833,81(1972) } \\
\text { NP A183,81 (1972) } \\
\text { NP A 183,81(1972) }\end{array}$ \\
\hline $\mathrm{Ca}$ & $\begin{array}{l}99.3 \\
179.6\end{array}$ & $\begin{array}{l}\text { CJP 44,1847(1966) } \\
\text { AF 19,527 (1961) }\end{array}$ \\
\hline Sc & 99.2 & CJP 44,1847 (1966) \\
\hline
\end{tabular}


Table VII (Cont.)

\begin{tabular}{|c|c|c|}
\hline Target & $\frac{\text { Energy }}{(\mathrm{MeV})}$ & Referencet \\
\hline $\mathrm{Ti}$ & 99.9 & CJP 44,1847 (1966) \\
\hline $\mathrm{V}$ & 99.1 & CJP 44, 1847 (1966) \\
\hline $\begin{array}{r}56 \mathrm{Fe} \\
\mathrm{Fe}\end{array}$ & $\begin{array}{l}60 \\
98.7 \\
179.6 \\
230 \pm 20 \\
345 \pm 20 \\
463 \pm 20 \\
552 \pm 20\end{array}$ & $\begin{array}{l}\text { PRL 22,1128(1969) } \\
\text { CJP 44,1847(1966) } \\
\text { AF } 19,527(1961) \\
\text { NP A } 183,81(1972) \\
\text { NP A } 183,81(1972) \\
\text { NP A } 183,81(1972) \\
\text { NP A } 83,81(1972)\end{array}$ \\
\hline $\begin{array}{r}59 \mathrm{Co} \\
\mathrm{Co} \\
58 \mathrm{Ni} \\
60 \mathrm{Ni} \\
\mathrm{Ni}\end{array}$ & $\begin{array}{l}60 \\
98.5 \\
60 \\
60 \\
98.5\end{array}$ & $\begin{array}{l}\text { PRL 22,1128 (1969) } \\
\text { CJP 44,1847(1966) } \\
\text { PRL 22,1128(1969) } \\
\text { PRL 22,1128 (1969) } \\
\text { CJP 44,1847(1966) }\end{array}$ \\
\hline $\mathrm{Cu}$ & $\begin{array}{l}77 \pm 5 \\
95 \pm 5 \\
99.0 \\
113 \pm 5 \\
133 \pm 5 \\
134 \pm 4 \\
225 \pm 20 \\
341 \pm \pm 20 \\
459 \pm 20 \\
548 \pm 20\end{array}$ & $\begin{array}{l}\text { NP 29,474(1962) } \\
\text { NP 29,474(1962) } \\
\text { CJP } 44,1847(1966) \\
\text { NP 29,474(1962) } \\
\text { NP 29,474 (1962) } \\
\text { PPS(Lond.) 67,125 (1954) } \\
\text { NP A183,81(1972) } \\
\text { NP A183,81(1972) } \\
\text { NP A183,81(1972) } \\
\text { NP A183,81(1972) }\end{array}$ \\
\hline $\begin{array}{r}68 \mathrm{Zn} \\
\mathrm{Zn}\end{array}$ & $\begin{array}{l}60 \\
99.1\end{array}$ & $\begin{array}{l}\text { PRL 22,1128 (1969) } \\
\text { CJP 44,1847 (1966) }\end{array}$ \\
\hline $\mathrm{Ge}$ & $\begin{array}{l}237 \pm 20 \\
347 \pm 20 \\
412 \pm 20 \\
498 \pm 20 \\
568 \pm 20\end{array}$ & $\begin{array}{l}\text { NP A183,81 (1972) } \\
\text { NP A } 183,81(1972) \\
\text { NP A } 183,81(1972) \\
\text { NP A } 183,81(1972) \\
\text { NP A } 183,81(1972)\end{array}$ \\
\hline $\begin{array}{r}90 \mathrm{Zr} \\
\mathrm{Zr}\end{array}$ & $\begin{array}{l}60 \\
98.8\end{array}$ & $\begin{array}{l}\text { PRL 22,1128 (1969) } \\
\text { CJP 44,1847(1966) }\end{array}$ \\
\hline $\mathrm{Nb}$ & 98.8 & CJP 44,1847 (1966) \\
\hline Mo & 99.1 & CJP 44,1847 (1966) \\
\hline $\mathrm{Pd}$ & 98.9 & CJP 44,1847 (1966) \\
\hline
\end{tabular}


Table VII (Cont.)

\begin{tabular}{|c|c|c|}
\hline Target & $\frac{\text { Energy }}{(\mathrm{MeV})}$ & Referencet \\
\hline $\mathrm{Ag}$ & $\begin{array}{l}77 \pm 5 \\
95 \pm 5 \\
98.3 \\
113 \pm 5 \\
133 \pm 5\end{array}$ & $\begin{array}{l}\text { NP 29,474(1962) } \\
\text { NP 29,474(1962) } \\
\text { CJP 44,1847(1966) } \\
\text { NP 29,474(1962) } \\
\text { NP 29,474(1962) }\end{array}$ \\
\hline $\mathrm{Cd}$ & $\begin{array}{l}98.8 \\
134 \pm 4\end{array}$ & $\begin{array}{l}\text { C.JP 44, } 1847 \text { (1966) } \\
\text { PPS(Lond.) 67,125 (1954) }\end{array}$ \\
\hline In & $\begin{array}{c}99.3 \\
179.6\end{array}$ & $\begin{array}{l}\text { CJP 44,1847(1966) } \\
\text { AF } 19,527(1961)\end{array}$ \\
\hline $\begin{array}{r}120 \mathrm{Sn} \\
\mathrm{Sn}\end{array}$ & $\begin{array}{l}60 \\
99.1 \\
221 \pm 20 \\
337 \pm 20 \\
456 \pm 20 \\
546 \pm 20\end{array}$ & $\begin{array}{l}\text { PRL } 22,1128(1969) \\
\text { CJP 44,1847(1966) } \\
\text { NP A183,81 (1972) } \\
\text { NP A 183,81 (1972) } \\
\text { NP A183,81 (1972) } \\
\text { NP A } 183,81(1972)\end{array}$ \\
\hline La & 98.8 & CJP 44,1847 (1966) \\
\hline Pr & 98.6 & CJP 44,1847 (1966) \\
\hline $\mathrm{Nd}$ & 98.6 & CJP 44,1847 (1966) \\
\hline Eu & 99.4 & CJP 44, 1847 (1966) \\
\hline Gd & 98.3 & $\operatorname{CJP} 44,1847$ (1966) \\
\hline $\mathrm{Tb}$ & 98.7 & CJP 44,1847 (1966) \\
\hline Dy & 98.2 & CJP 44,1847 (1966) \\
\hline Ho & 99.1 & CJP 44,1847 (1966) \\
\hline $\mathrm{Tm}$ & 99.3 & CJP 44,1847 (1966) \\
\hline Hf & 98.8 & CJP 44,1847 (1966) \\
\hline $\mathrm{Ta}$ & 98.3 & CJP 44,1847 (1966) \\
\hline W & 99.3 & CJP 44,1847 (1966) \\
\hline $\operatorname{Re}$ & 99.0 & CJP 44,1847 (1966) \\
\hline $\mathrm{Pt}$ & 99.3 & CJP 44,1847 (1966) \\
\hline
\end{tabular}


Table VII (Cont.)

\begin{tabular}{|c|c|c|}
\hline Target & $\frac{\text { Energy }}{(\mathrm{MeV})}$ & $\underline{\text { Referencet }}$ \\
\hline $\mathrm{Au}$ & $\begin{array}{l}99.4 \\
179.6\end{array}$ & $\begin{array}{l}\text { CJP 44,1847(1966) } \\
\text { AF } 19,527(1961)\end{array}$ \\
\hline $\mathrm{Tl}$ & 99.6 & CJP 44,1847 (1966) \\
\hline $\begin{array}{r}208 \mathrm{~Pb} \\
\mathrm{~Pb}\end{array}$ & $\begin{array}{l}60 \\
77 \pm 5 \\
95 \pm 5 \\
99.2 \\
113 \pm 5 \\
133 \pm 5 \\
134 \pm 4 \\
226 \pm 20 \\
341 \pm 20 \\
460 \pm 20 \\
549 \pm 20\end{array}$ & $\begin{array}{l}\text { PRL 22,1128 (1969) } \\
\text { NP 29,474 (1962) } \\
\text { NP 29,474 (1962) } \\
\text { CJP 44,1847(1966) } \\
\text { NP 29,474(1962) } \\
\text { NP 29,474 (1962) } \\
\text { PPS(Lond.) 67,125 (1954) } \\
\text { NP A183,81 (1972) } \\
\text { NP A183,81 (1972) } \\
\text { NP A183,81 (1972) } \\
\text { NP A183,81 (1972) }\end{array}$ \\
\hline Th & 99.1 & CJP 44,1847 (1966) \\
\hline $\mathbf{U}$ & 98.9 & CJP 44,1847 (1966) \\
\hline +Joumal Abbreviation: & \multicolumn{2}{|c|}{$\begin{array}{l}\text { PRL - Physical R.eview Letters } \\
\text { CJP - Canadian Journal of Physics } \\
\text { PPS(Lond.) - Proceedings of the Physcial } \\
\text { Society (London) } \\
\text { NP - Nuclear Physics } \\
\text { AF - Arkiv for Fysik }\end{array}$} \\
\hline
\end{tabular}

